\title{
Personality disorders : inquiries into assessment, cognitive profiles and impact on treatment of anxiety disorders
}

Citation for published version (APA):

Dreessen, L. J. J. M. (1998). Personality disorders : inquiries into assessment, cognitive profiles and impact on treatment of anxiety disorders. [Doctoral Thesis, Maastricht University]. Datawyse / Universitaire Pers Maastricht. https://doi.org/10.26481/dis.19980918ld

Document status and date:

Published: 01/01/1998

DOI:

10.26481/dis.19980918ld

Document Version:

Publisher's PDF, also known as Version of record

Please check the document version of this publication:

- A submitted manuscript is the version of the article upon submission and before peer-review. There can be important differences between the submitted version and the official published version of record.

People interested in the research are advised to contact the author for the final version of the publication, or visit the DOI to the publisher's website.

- The final author version and the galley proof are versions of the publication after peer review.

- The final published version features the final layout of the paper including the volume, issue and page numbers.

Link to publication

\footnotetext{
General rights rights.

- You may freely distribute the URL identifying the publication in the public portal. please follow below link for the End User Agreement:

www.umlib.nl/taverne-license

Take down policy

If you believe that this document breaches copyright please contact us at:

repository@maastrichtuniversity.nl

providing details and we will investigate your claim.
}

Copyright and moral rights for the publications made accessible in the public portal are retained by the authors and/or other copyright owners and it is a condition of accessing publications that users recognise and abide by the legal requirements associated with these

- Users may download and print one copy of any publication from the public portal for the purpose of private study or research.

- You may not further distribute the material or use it for any profit-making activity or commercial gain

If the publication is distributed under the terms of Article $25 \mathrm{fa}$ of the Dutch Copyright Act, indicated by the "Taverne" license above, 
Personality Disorders 
ISBN $90-5278-239-3$

Universutaire Pers Masstricht

(B. Dreessen. Maastricht 1998

Omslagontmerp: Datawyse Maastricht Ontwerp binnerwwerk: Sandira Datems

Mustratias: Sandra Daems

Drukwerk: Datawyse Maastricht 


\section{Personality Disorders}

Inquiries into Assessment, Cognitive Profiles and Impact on Treatment of Anxiety Disorders

(Persoonlijkheidsstoomissen: onderzoeken narar diagnostiek, cognitieve profielen en inolod op behandeling anm angststoonissen)

\section{PROEFSCHRIFT}

ter verkrijging van de graad wan doctor aan de Universiteit Maastricht, op gezag van de Rector Magnificus,

Prof. dr. A.C. Nieuwenhuijzen Kruseman, volgens het besluit van het College van Decanen, in het openbaar te verdedigen op vrijdag 18 september 1998 om 14.00 uur

door

Laura Josephina Johanna Maria Dreessen

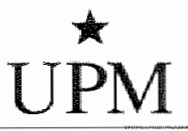

UNIVERSITARE PERS MAASTRICHT 


\section{Promitotor}

Prof. dr. M.A. van den Hout

Co-prowotor

Dr. A.R. Arntz

Beoordelingscommissie

Prof. dr. H. Merckelbach (voorzitter)

Prof. dr: W. van den Brink (Amsterdam Institute for Addiction Research)

Prof. dr. H.M. van Praag

Dr. C. de Ruiter (Dr. Henri van der Hoeven Kliniek)

Prof. dr. H.G. Schmidt 


\section{Contents}

$\begin{array}{ll}\text { Preface } & 7\end{array}$

Chapter 1 General Introduction 9

Chapter 2 Assessment of Personality Disorder:

The Structured Clinical Interview for DSM-III-R

Personality Disorders (SCID-II)

2.1. Short-interval test-retest interrater reliability of the Structured Clinical Interview for DSM-Im-R Personality Disorders (SCID-II) in outpatients

2.2 Patient-informant concordance on the Structured Clinical Interview for DSM-II-R Personality Disorders (SCID-I)

Chapter 3 The Impact of Personality Disorders on Cognitive and Behavior Therapies for Anxiety Disorders

3.1 Personality disorders do not influence the results of cognitive and behavior therapies for panic disorder and for various anxiety disorders lumped together as a group

3.2 Personality disorders do not influence the results of cognitive and behavior therapy for obsessive compulsive disorder

3.3 Personality disorders have no excessively negative impact on the therapy process in cognitive and behavioral treatment of Axis I anxiety disorders

Chapter 4 The Impact of Personality Disorders on Treatment Outcome of Anxiety Disorders: Best-Evidence Synthesis

Chapter 5 Cognitive Profiles of Personality Disorders

5.1 Assessment of cognitive profiles in personality disorders: development of the Personality Disorder Belief Questionnaire (PDBQ)

5.2 Avoidant personality disorder and schema-congruent information processing bias: a pillot study with a pragmatic inference task

Chapter 6 General Discussion: Implications from and for Research 
References

Summary

171

Samenvatting

175

Dankwoord

Curriculum Vitae 


\section{Preface}

The present thesis comprises several empirical studies with personality disorders being the central topic. Chapter 1 is intended as a general introduction. Relevant issues on definition, assessment, theory and treatment are touched on. The Diagnostic and Statistical Manual of Mental Disorders (DSM) is put forward as the official nomenclature. Next to it, emphasis is laid on the cognitive theory of personality disorders, which is developing into one of the main theoretical orientations in the personality disorder literature. As far as treatment is concerned, Chapter 1 focuses on the hypothesized interference of personality disorders in the treatment of anxiety disorders. The chapter is closed with the research questions addressed in this thesis.

In Chapter 2, two studies are presented on the assessment of personality disorders by the Structured Clinical Interview for DSM-III-R Personality Disorders (SCIDII) $y_{x}$ a frequently used and well-known instrument. The first study concerns the short-interval test-retest interrater reliability (section 2.1) and in the second study convergent validity is tested by comparing patient based and informant based diagnoses (section 2.2).

In Chapter 3, four studies are presented in which the impact of personality disorders on cognitive and behavior therapies for various anxiety disorders was investigated. Three studies focus on therapy outcome and one on therapy process. Section 3.1 deals with therapy outcome in panic disorder and in various anxiety disorders lumped together as a group. Section 3.2 deals with therapy outcome in obsessive compulsive disorder. The fourth study in section 3.3 addresses the impact of personality disorders on the therapy process in the treatment of various Axis I anxiety disorders lumped together as a group.

In Chapter 4 , an up to date overview is given of studies concerning the impact of personality disorders upon the treatment outcome of anxiety disorders. To our knowledge, data of 35 studies on this issue have been presented since 1965. Welldesigned studies are discussed in detail, resulting in a best-evidence synthesis.

In Chapter 5 , a recently developed self-report questionnaire is introduced, the so-called Personality Disorder Belief Questionnaire (PDBQ), which attempts to identify maladaptive cognitive profiles (i.e. beliefs) that correspond with the DSM personality disorders (section 5.1). Furthermore, the first study in a series of studies designed to test the cognitive model of personality disorders, is presented. It is tested whether the DSM-III-R avoidant personality disorder is characterized by biased information processing governed by typical maladaptive schemas (section 5.2).

In the last chapter, the main findings of the presented studies are discussed. The present thesis is closed by recommendations and suggestions for further research on personality disorders. The majority of the sections of this thesis were submitted for publication as separate manuscripts and therefore some overlap was inevitable. 



\section{General introduction}

\section{A case example}

A young woman has a first appointment with a psychotherapist. She complains about having panic attacks with fear of dying. She worries about the panic attacks, and also about other topics. Furthermore, she has difficulties to drop off to sleep and her sleep is shallow. During the day, she is without any energy and her ability to concentrate is weak. She feels regularly gloomy. Later in the session, she talks about being afraid to make mistakes, to be very insecure a lot of times, and to be afraid to take on commitments. She is preoccupied making a good impression on others. She is afraid others will judge her being stupid. Apart from being very self-critical, she is also very critical to others. Moreover, she feels exploited very easily. She remembers herself being an anxious child, afraid to do wrong. She describes herself as being a worrywart all her life. At first, she did not comply with the advice of the family doctor to apply for help to the Community Mental Health Center. A further increase in acute complaints made her change her mind. A comprehensive analysis of present and past problems followed.

Diagnostic interviews, using the nomenclature of the third revised edition of the Diagnostic and Statistical Manual of Mental Disorders (DSM-MI-R, American Psychiatric Association, 1987), indicated the presence of a panic disorder without agoraphobia, a social phobia and a depressive disorder Not Otherwise Specified (Axis I) Furthermore, several personality disorders (PDs) were diagnosed (Axis II). She met the criteria for an obsessive compulsive, an avoidant, and a paranoid personality disorder.

The present case leads to various questions: What treatment strategies should be chosen? Do we start with treatment for the Axis I disorders or with treatment for the PDs? Can we treat the Axis I disorders without treating the PDs? Can we expect difficulties in the treatment of the Axis I disorders because of the presence of concomitant PDs? How reliable and valid are the PD diagnoses? Do the current anxiety and depressive disorders distort the assessment of PDs? The present thesis will give some answers.

\section{Personality disorders according to the diagnostic and statistical manual of mental disorders}

In 1980 the third edition of the DSM (APA, 1980) was published. In contrast to the DSM-II (APA, 1968), it included specific diagnostic criteria for PDs. Moreover, a separate Axis for PDs was introduced (Axis II) and multiple PD diagnoses were allowed. In that way, it was promoted to draw a distinction between lifelong and egosyntonic disorders of personality functioning and episodic and egodystonic so-called symptom disorders, such as anxiety disorders or psychotic disorders 
(Axis 1). These changes have had an enormous effect on the level of interest in PDS among clinicians and researchers. First, a number of assessment instruments have been developed that greatly improved diagnostic reliability. Second, the multiaxial system has promoted interest for the fundamental issues that underlie the domain of PDS, for the relation with Axis I disorders, and for the impact of PDS on treatment of Axis I disorders. The DSM-III system of diagnosing PDs is being retained in the DSMPV (APA, 1994) and therefore it will continue to influence psychiatric thinking for quite a number of years.

According to the DSM-IV (APA, 1994, p. 629) a PD is 'an enduring pattern of inner experience and behavior that deviates markedly from the expectations of the individual's culture, is pervasive and inflexible, has an onset in adolescence or early adulthood, is stable over time, and leads to distress or impairment".

Ten specific PDs are distinguished, each characterized by a set of maladaptive traits. The DSM-IV (and the DSM-III-R) uses a polythetic format, which means that not all, but only a specified number of the specific maladaptive traits is required for diagnosing a specific PD (e.g. 4 out of 7 avoidant traits). Consequently, heterogeneity within PD categories can be expected. Note that a poly thetic format is also used for most of the Axis I disorders. The ten PDs are grouped into three clusters. Cluster A. (called the odd cluster) includes the paranoid, schizoid, and schizotypal PDs. Cluster B (called the dramatic cluster) includes the antisocial, histrionic, borderline and narcissistic PDs. Cluster C (called the anxious cluster) includes the avoidant, dependent, and obsessive compulsive PDs. Disorders of personality functioning that do not meet criteria for any of the ten aforementioned PDs, are to be categorized as PD-Not Otherwise Specified (PD-NOS). Examples are mixed $\mathrm{PD}$, depressive $\mathrm{PD}$, passive-aggressive $\mathrm{PD}$, self-defeating $\mathrm{PD}$ and sadistic $\mathrm{PD}$. Depressive and passive-aggressive PDs are categories suggested to be included in a future classification. Note that passive-aggressive PD is degraded from being a classified category in the DSM-III-R to a suggested category in the DSM-IV. Selfdefeating and sadistic PD were suggested categories in the DSM-III- $R$ which are not mentioned anymore in the DSM-IV. A short characterization of every DSM-III-R and DSM-IV PD category is given in Table 1.

\section{Assessment of personality disorders}

For the assessment of pos, self-report questionnaires and interviews [(semi)structured and unstructured] are being used. Examples of the most widely used instruments are the Personality Diagnostic Questionnaire-Revised (PDQ-R: Hyler, Skodol, Kellman, Oldham, \& Rosnick, 1990), the Millon Clinical Multiaxial Inventory (MCM1-ill: Millon, 1987), the Structured Clinical Interview for DSM-III-R Personality Disorders (SCID-II: Spitzer, Williams, Gibbon, \& First, 1990), the Structured Interview for DSM-III-R Personality Disorders (SIDP: Stangl, Pfohl, Zimmerman, Bowers, Corenthal, 1985), and the Personality Disorder Examination (PDE, Loranger, Susman, Oldham, \& Russakoff, 1987). The interviews have been updated for the DSM-IV (First, Spitzer, Gibbon, Williams, \& Benjamin, 1994; Pfohl Blum, \& Zimmerman, 1995; Loranger et al., 1994, respectively). For a short description of several instruments the reader is referred to reviews by Van Velzen and Emmelkamp (1996) and Widiger and Frances (1987). Below, various difficulties in the assessment of PDs are discussed. 


\section{TAELE 1}

Characterization of the DSM-IV and DSM-PHI-R personality disorders (classified and suggested categories)

Personality Disorder

Paranoid

Schirzoid

Schizotypal

Antisocial

Borderline

Histrionic

Narcissistic

Avoidant

Dependent

Obsessive Compulsive

Depressive

Passive-aggressive ab

Self-defeating

Sadistivic

a Suggested category in DSM-IV

b Classified category in DSM-HI-R

C. Suggested category in DSM-III-R

First of all, the diagnostic criteria leave a lot of room for subjective judgment. It has to be decided whether traits and the corresponding PDs are severe enough to be maladaptive (i.e. causing distress or impairment), whether they are pervasive enough across a broad range of situations and whether they are stable enough to characterize the long-term functioning (DSM-III-R/DSM-IV, 1987, 1994).

The problem to distinguish between traits in the normal range and traits in the pathological range (i.e. severe enough to cause distress or impairment) has raised a fundamental question in the assessment of PDS, namely whether categorical or dimensional scales of measurement should be used in diagnosing traits and in diagnosing PDs (Widiger, 1992). The categorical perspective, as is used in the DSMs, 
assumes that there is a clear distinction between the presence and the absence of a $\mathrm{PD}$ (or a trait). The definition of the DSM-II-R/TV, that a maladaptive, pervasive and stable pattern of traits has to cause significant distress or impaiment, could provide the rationale for the diagnostic threshold, but it does not provide the rationale that was used for the DSM-III-R/Y cutoff points (Widiger, 1992). For example, four maladaptive, pervasive and stable dependent traits may well cause significant distress or impairment, but at least five traits are required for diagnosing a dependent PD. According to the dimensional perspective, PDS represent maladaptive variants of personality traits that merge imperceptibly into normality. Whereas the categorical approach is more familiar to clinicians proceeding from a medical tradition, the dimensional approach (e.g. the total number of traits) has been found to result in more reliable and valid ratings (see Widiger, 1992, p. 293). The implications for research are obvious: researchers should use more dimensional assessments, at least in addition to categorical diagnoses. Research concerned with correlates of PDs (e.g. etiology, treatment outcome) will be more powerful and informative using dimensional assessments.

A second problem in the assessment of PDs is the simultaneous presence of Axis I disorders. The acute psychiatric state might cause biases in the report of information. It is well-known that a state of depression or anxiety can bias the memory of past experiences (Williams, Watts, Macleod, \& Mathews, 1988). Anxiety and depression may also be accompanied by cognitive distortions that affect judgment of the self (Stein, Hollander, \& Skodol, 1993). Furthermore, the DSM-IIIR/IV warns us for the temporary changes in personality functioning due to Axis I conditions that may look like some of the maladaptive personality traits, but that should not be diagnosed as Axis Ir traits. The last is easier said than done. Especially in cases with long-lasting Axis I disorders it is sometimes difficult to distinguish between 'real' personality and temporary changes in personality functioning resulting from an enduring $A x i s$ I disorder.

A third difficulty in the assessment of PDs concerns lack of insight in the patient (ego-syntonicity) and underreport due to shame. Therefore, patients may not be the optimal source of information (also because they may have difficulty distinguishing state from trait symptoms, as was mentioned above). A close friend or relative (so-called informants), who has observed the patient over a fairly long period, may be a more accurate source of information in the assessment of PDs. Several studies have reported poor or only modest concordance between patient and informant reports (Bernstein et all, 1997; Dowson, 1992; Riso, Klein, Anderson, Oumette, \& Lizardi, 1994; Steketee, 1990; Tyrer, Alexander, Cicchetti, Cohen, \& Remington, 1979; Zimmerman, Pfohl, Coryell, Stangl, \& Corenthal, 1988) and, so $\mathrm{far}_{3}$ no attempt was made to determine which of these two sources of information is more valid.

The main difficulty in testing validity of $P D$ instruments ${ }^{1 /}$ is the lack of a golden standard. Spitzer (1983) proposed that a LEAD standard should be used as a near golden standard against which to compare various structured instruments for mental disorders. The LEAD standard refers to a Longitudinal Expert evaluation that uses All Data. The patient is evaluated not just once but over a period of time. Expert clinicians, who have demonstrated their ability to make reliable diagnoses, make independent diagnoses, based on thorough clinical interviews. They have access to all available information that might be informative, including reports 
from treating clinicians or staff, family members and friends. Finally, the experts discuss the reasons for diagnostic disagreement, and then make consensus diagnoses.

Two comments need to be made regarding the LEAD procedure. First, Spitzer (1983) stressed that each expert clinician should have previously participated in a systematic diagnostic reliability study in which adequate reliability was attained. Since several studies (Mellsop, Varghese, Joshua, \& Hicks, 1982; Pilkonis, Heape, Ruddy, \& Serrao, 1991; Shea et al., 1990; Spitzer, Forman, \& Nee, 1979) demonstrated poor reliability of unstructured clinical PD evaluations, the experts have to show the ability to make reliable diagnoses by using semistructured PD interviews.

The second comment concerns the psychometric properties of the LEAD procedure itself. The reliability of the LEAD standard for PDS is far from being properly tested. Only one study (Kranzler, Kadden, Babor, \& Rounsaville, 1994) could be traced. This study reported on the reliability of only two PDs using the LEAD procedure (Kappas: borderline $\mathrm{PD}=0.66$, antisocial $\mathrm{PD}=0.40$ ). If it turns out that the LEAD diagnoses cannot be made reliable, it cannot serve as a standard of comparison. A poor concordance between the LEAD procedure and diagnostic instruments may be due to poor reliability of the LEAD diagnoses, or it may be due to validity problems of the instruments. In short, the reliability of the LEAD standard for PDS should be evaluated in a variety of patient groups before it can be accepted as a criterion measure against which diagnostic instruments can be compared.

Given the difficulties mentioned above, which measurement instrument is preferable for the assessment of PDs (apart from the sources of information)? Subjective judgment of the diagnostic criteria in terms of severity, pervasiveness, and stability can be trained in raters who administer interviews, but not in patients who fill out self-report questionnaires. Similarly, influences of state factors and the separation of state from trait symptoms are believed to be more under control in interviews held by trained raters than in self-report questionnaires (Dreessen \& Arntz, 1998-a; Widiger \& Frances, 1987, p. 67; Zimmerman, 1994, p. 237). The fact that self-report questionnaires report higher levels of PD pathology in clinical samples than (semi)structured clinical interviews (e.g. Hunt \& Andrews, 1992; Hyler et al., 1990; Hyler, Skodol, Oldham, Kellman, \& Doidge, 1992; Reich \& Noyes, 1987) seems to support this notion. Unstructured clinical interviews contain the risk of underreport, because clinicians might stick to the one or two disorders they have identified. (Semi)Structured interviews, on the contrary, guide the clinical examination so that all relevant areas are covered. Moreover, (semi)structured interviews have been proven to result in reasonable reliable PD diagnoses (see review by Zimmerman, 1994, p. 226-227), whereas reliability of unstructured interviews has been found to be relatively low (e.g. Mellsop et al., 1982). In conclusion, (semi)structured clinical interviews can be considered to be the most reliable and valid method in diagnosing PDs (Dreessen \& Arntz, 1998-a). Self-report questionnaires may be useful in screening for personality pathology, rather than diagnosing personality pathology (Zimmerman, 1994). Standardized instruments for the assessment of PDS need to be further tested for reliability and validity. Section 2.1 and 2.2 of this thesis report on a reliability study and a validity study of a well-known and frequently used semistructured interview for PDs.

The case at the beginning illustrates that PDS often co-occur with anxiety dis- 
orders. Apart from diagnostic issues, as discussed above, clinically important questions arise about the incidence of comorbid PDS given an anxiety disorder and the implications of comorbid PDs for the treatment of anxiety disorders. These issues will be dealt with in the next paragraph.

\section{Anxiety disorders and personality disorders}

The prevalence of one or more DSM-II (-R) PDS (APA, 1987) has been reported to be around $10 \%$ in the general population (Zimmerman \& Coryell, 1989; Maier, Lichtermann, Klinger, Heun, \& Hallmayer, 1992). In groups of patients with an Axis I anxiety disorder, the prevalance rates are found to be much higher. Van Velzen \& Emmelkamp (1996) summarized studies on prevalence of PDs in patients with anxiety disorders. The median prevalence rates of one or more PDs with panic disorder (with or without agoraphobia), social phobia, generalized anxiety disorder, and obsessive compulsive disorder varied between $50-60 \%$. The avoidant, dependent, and obsessive compulsive PDs (Cluster C) were the most prevalent PDs in these four anxiety disorders. More specifically, generalized social phobia is often (but not perfectly) associated with avoidant PD (e.g. Holt, Heimberg, \& Hope, 1992; Jansen, Arntz, Merckelbach, \& Mersch, 1994; Schneier, Spitzer, Gibbon, Fyer, \& Liebowitz, 1991). Panic disorder is often associated with dependent (e.g. Reich, Noyes, \& Troughton, 1987) and avoidant PD (e.g. Green \& Curtis, 1988). Contrary to the expectation, obsessive compulsive disorder is often not specifically associated with obsessive compulsive $\mathrm{PD}$. For example, Black and colleagues (1989) found that none of the 21 patients with obsessive compulsive disorder had an obsessive compulsive PD. Baer and colleagues (1990) found that only 6 of the 96 patients with obsessive compulsive disorder had an obsessive compulsive PD. These frequencies are more or less comparable to the frequencies of obsessive compulsive PD found in patients with other anxiety disorders (e.g. Van Velzen, Emmelkamp, \& Scholing, 1997: 5/61 social phobia; Green \& Curtis, 1988: $3 / 25$ panic disorder; Sanderson, Beck, \& McGinn, 1994: 1/32 generalized anxiety disorder).

Reported prevalences of PDS in anxiety disorder samples vary enormously, which is probably due to the selection of the samples (e.g. outpatients vs. inpatients) and to the type of assessment instrument that is used for diagnosing pDs (e.g. semistructured interview vs. self-report questionnaire). More severe Axis 1 or Axis II pathology is usually characteristic for inpatient settings. Self-report questionnaires generally report higher PD rates than (semi)structured interviews (e.g. Reich \& Noyes, 1987; Renneberg, Chambless, Dowdall, Fauerbach, \& Gracely, 1992). But even the lowest prevalence estimates indicate that there are a lot of patients with anxiety disorders who have one or more concomitant PDS.

The relationship between anxiety disorders and PDs may be conceptualized in five comorbidity models, which are not mutually exclusive (e.g. Stein et al., 1993). First, PDs may act as a vulnerability factor increasing the likelihood of occurrence (quantity) of particular anxiety disorders. Second, PDs may act as a pathoplastic factor modifying the symptomatology (quality) of particular anxiety disorders (e.g. specific phenomenology or course). Third, pDs may be a consequence of enduring anxiety disorders. Fourth, PDS and anxiety disorders may arise from a common cause, or they may have different etiologies, that interact. Fifth, PDs and 
anxiety disorders may co-occur because of the overlap in symptomatology (e.g. avoidant PD and social phobia). Although some specific hypotheses have been generated (e.g. dysfunctional midbrain and neocortical structures as the underlying predisposition to both anxiety disorder and Cluster C PDs: Gorman, Liebowitz, Fyer, \& Stein, 1989), empirical validation of the above mentioned comorbidity models is lacking (Stein et al., 1993).

A clinically important question concerns the influence of comorbid PDs on the treatment process and outcome of Axis I anxiety disorders. From the comorbidity models and from the nature of PDS several hypotheses can be generated for PDs to interfere with the treatment of anxiety disorders (hypotheses are also applicable to other Axis I disorders):

1 According to the vulnerability model, treatment of the anxiety disorder without treating the underlying PD will result in displacement of symptoms or relapse of the anxiety disorder.

2 The pathoplastic model predicts qualitative differences within the anxiety disorder categories which might complicate the treatment. For instance, in patients with PDS, Axis $\mathbb{I}$ avoidance behavior may be more severe and rigid, loss of control in panic disorder may be more overwhelming, worrying in generalized anxiety disorder may be more excessive, or the content of cognitions and schemas in Axis I anxiety disorders may be typical (e.g. fear of dying in panic disorder without PDS and fear of going made or losing control in patients with PDs).

3 The other comorbidity models do not posit a quantitative or qualitative effect of PDS on Axis I anxiety disorders, but just from the nature of PDS hypotheses can be generated about interference of PDS in the Axis I therapeutic relationship and in the Axis I therapy compliance. Such problems may result in less well outcome in the treatment of anxiety disorders (or any other Axis I disorder). The problems are for instance distrust towards the therapist, fear of disapproval or rejection by the therapist, fear to disagree with the therapist, fear of being controlled and dominated by therapist, fear to take initiatives oneself, fear to take any risks, underestimation of own capacities, low tolerance for strong emotions, poor compliance with homework assignments, and early termination of therapy.

On the face of it, the above mentioned hypotheses seem very plausible and one would expect a complicated Axis I therapy process and poorer Axis I therapy outcome. Indeed, in clinical practice, failures in therapy are easily attributed to the presence of PDS. Moreover, patients with PDS run the risk of being excluded from a serious. Axis I therapy from the very start. Studies on the impact of PDs on the Axis I therapy process could not be traced. On the other hand, numerous studies were traced on the impact of PDs on therapy outcome of Axis I anxiety disorders. A first series of studies on the impact of PDS on the therapy outcome of Axis I anxiety disorders seemed to confirm the idea of PDS being a negative predictive factor (see Appendix 1, Chapter 4 of the present thesis). However, these studies suffer from several methodological limitations, such as retrospective PD diagnoses without being blind to Axis I treatment outcome (e.g. Numberg et al., 1989; Tumer, 1987) or use of questionable assessment instruments for diagnosing PDs (e.g. Minichiel]o, 
Baer, \& Jenike, 1987; Vaughan \& Beech, 1985). In the 90s, a new series of studies has been conducted in which methodology is improved (e.g. studies presented in section 3.1 and 3.2 of the present thesis): PDs are assessed prior to the treatment by independent semistructured interviews. Moreover improvement is not only studied in terms of end-state functioning, but also in terms of change with respect to pretest scores. Even if patients with PDS cannot reach the end-state functioning in anxiety symptoms of patients without a PD, it would be of great clinical importance to demonstrate that they can profit equally from a standardized treatment in terms of amount of improvement. The question arises whether or not one can continue to conclude from all the available evidence that, in general, PDs affect treatment outcome of anxiety disorders negatively (see Chapter 4 of the present thesis).

In case PDS are found to be unrelated to treatment outcome of Axis I anxiety disorders, it still leaves unanswered the question whether or not PDs complicate the Axis I therapy process. Possibly, complication by PDS in an Axis I therapy can be handled successfully without resulting in poorer Axis I treatment outcome at the end. As mentioned above, no study could be traced investigating the impact of PDs on the Axis I therapy process directly (section 3.3. of the present thesis offers a first study). So far, it can only be assumed that PDs have a negative impact on the therapeutic relationship or on other Axis I therapy process variables.

\section{Cognitive theory of personality disorders}

The cognitive theory of PDs is developing into one of the main theoretical orientations in the PD literature (Klein, 1993; Shea, 1993) and is of great clinical interest. First of all, the theory explains the etiology and maintenance of PDS and offers a treatment model for PDs, the so-called schema focused therapy (Beck, Freeman, \& Associates, 1990; Padesky, 1994; Young, 1990). Secondly, by offering cognitive profiles, the theory helps us to understand the way patients with PDS react, emotionally and behaviorally, to various circumstances, including an Axis I therapy. In that way the cognitive theory indicates specific problems to be expected in the treatment of Axis I disorders and in what way these problems may successfully be dealt with. Below, the basic premises of the cognitive theory are outlined.

In general, cognitive theory (Beck, 1976; Markus \& Zajonc, 1985; Padesky, 1994) assumes that each person has cognitive schemas. Cognitive schemas are theoretical constructs referring to global, mostly implicit knowledge structures acquired throughout life. Schemas are presumed to direct the processing of information (attention/selection, interpretation, memory) to a large extent, resulting in thoughts, affect and behavior. The influence of schemas on the processing of information is mostly automatic instead of conscious.

Cognitive schemas can operate on the information processing in an adaptive or maladaptive way $(c$. functional or dysfunctional). It is hypothesized that maladaptive schemas result in extreme and biased selection, interpretation and memory of information. These so-called biases or cognitive distortions are believed to underlie emotional and behavioral problems, including PDs. Moreover, the biases are schema-confirming and that way pathology is maintained. 
The cognitive model for PDS (Beck et al., 1990) assumes that each PD is characterized by typical maladaptive (c\%. dysfunctional) schemas of the self and the (social) world. Note that the content of schemas is mostly implicit. The explicit formulation of the content of schemas is called "belief' or 'assumption" (e.g. "I am helpless"; 'Others are very competent'; 'If I am abandoned, I cannot function anymore"). PD schemas are believed to have their origin in childhood or in early adolescence. Several factors may contribute to the development of PD schemas:

1 innate characteristics that become reinforced by subsequent experience (e.g. shyness that evolves into an avoidant PD; Beck et al. 1990). There is evidence that certain types of temperaments and behavioral patterns are present at birth (Kagan, 1989);

2 interaction with significant others, such as the parents, siblings or peers, who model and reinforce behavior, emotions and beliefs (Beck et al., 1990);

3 intrusive events, such as traumatic experiences (Weaver \& Clum, 1993; Arntz, Dietzel, \& Dreessen, 1997).

The newly-developed maladaptive schemas lead to schema-confirming cognitive distortions that strengthen the schema over and over again. Moreover, extreme emotions and behaviors that go together with the schemas, can elicit reactions from the environment which are also schema-confirming (e.g. a child that is being punished or rejected for showing fear). The early origin of PD schemas implies a succession of schema-confirming experiences and a lack of scherna-disconfirming experiences. Consequently, the maladaptive PD schemas are believed to be overgeneralized, inflexible, imperative and difficult to change (Beck et al, 1990 , Padesky, 1994). Adaptive personality schemas are underdeveloped and one cannot appeal to them (it is assumed that in Axis I disorders, adaptive schemas are latently present, but temporarily not available). Therefore, in schema-focused PD therapy, effort is put in developing new adaptive schemas, and weakening the old maladaptive ones (Padesky, 1994). In schema-focused PD therapy, the person is being asked to go far beyond changing a particular behavior or reframing a particular interpretation, as is done in usual Axis I therapy. They are being asked to give up who they are and how they have defined themselves for many years (Beck et al., 1990, p. 9).

As was already mentioned above, each $P D$ is believed to be characterized by a typical set of schemas, the so-called cognitive profile (Beck et al., 1990). Schemas contain mainly implicit knowledge, which is not directly observable for others or for the self by means of introspection, but which does result in observable output, such as thoughts, affects and behavior. From this output, implicit schemas can be derived and be made explicit verbally. The explicit formulation of the content of schemas is called 'belief' or 'assumption'. Across PD categories, three types of beliefs are distinguished (Beck et al., 1990): core or basic beliefs, conditional beliefs, and self-instructional or instrumental beliefs. Core beliefs are the most fundamental ones and they refer to the view of the self and others (e.g. 'I am fully responsible', 'Others are irresponsible'). The core beliefs feed into the next level of conditional beliefs, which refer to 'If..., then...' reasonings (e.g. 'If something goes wrong, then it is my fault'). The self-instructional beliefs refer to specific do's and don'ts, imposed to oneself in order to prevent alleged troubles or misery (e.g. 'Be per- 
fect!') or to get what is believed to be good (e.g. "Take it!'). These rules represent general survival strategies. The cognitive profiles of the DSM-IV PDS are presented in Table 2.

TABLE 2

Cognitive profiles of the DSM-IV personality disorders

\begin{tabular}{|c|c|c|}
\hline $\begin{array}{l}\text { Personality } \\
\text { disorder }\end{array}$ & Bellofs of the selt a & $\begin{array}{l}\text { Betiefs about } \\
\text { others a }\end{array}$ \\
\hline paranoto & $\begin{array}{l}\text { - I am honest, in- } \\
\text { mocent. vulmerables, } \\
\text { and mistreated }\end{array}$ & $\begin{array}{l}\text { Others cannot be } \\
\text { trusted } \\
\text { "Others are mis- } \\
\text { lleading and out to } \\
\text { undermine me }\end{array}$ \\
\hline
\end{tabular}

$\begin{array}{ll}\text { Schizoid } & -1 \text { do not need } \\ & \text { other people } \\ & -1 \text { am better off on } \\ & \text { my own } \\ \text { Schizotypal } & -1 \text { can feel it when } \\ & \text { bad things will } \\ & \text { happen } \\ & -1 \text { know what } \\ & \text { others thimk } \\ & -1 \text { do inot really } \\ & \text { exist }\end{array}$

- Others are intrinsilve

- Others will restrict my freedom trusted

- Others will get you

if they can

- Other cen fied iny feelings
- Others cannot be

\section{Conditional beliefs a}

- ll am not careful. others will manipLilate me or tathe advantage of me - If people act friendly, they are trying to use me - If people seem distant it proves they are against me

- If I get loo close to people. I will lose my freedom

- If amything hap. pens around me, it predicts my or ather's, (nearl

future

- If I think or fee bad things, they will happen

- If peoplle act friendly, they are Hrying to use me - If people seem distant, it proves they are against me

Antisocial

- Iam strong
- I can take care of
myself
- I deserve more
than l gat, sol am
entitled to break
rules

Borderline -I am an avil perrson and I deserve purishmont - I am helpless and vulnerable sol rieed others to take care of me

\footnotetext{
- Others cannot be trusted

- Others are exphlotative, and thus dieserving of baing exploited

- Others ane wealk. and thus deserving of being proyed on

- Others are malewolent, abusing. rejecting and untrustworthy - Others do not really care for me
}
- Ir ll am not the ag- gressor, I will be the wictim
- If I don"t push others around, I will never get what : deserve

Self-insitructional beliefs a

- Be on guard!
- Donit trust
anybodyl
- Look for hidden
motives!

- Don't get too close!

- Don't get involved!

\section{- Be on guard for danger \\ - Look for hidden signs! \\ - Do not think or feel bad things!}

\author{
Get the other: \\ - Take it! \\ - Ut is your turn nowl
}

\author{
- If I trust the other, I Find support! \\ will get disappoin - Don't trust anyonel \\ ted \\ - If I comply with \\ someone's request, \\ I run the risk of llo- \\ sing myself \\ - If I refusie some- \\ onve"s request, $\|$ run \\ the risk of losing \\ this person
}


Table 2 fcontinued

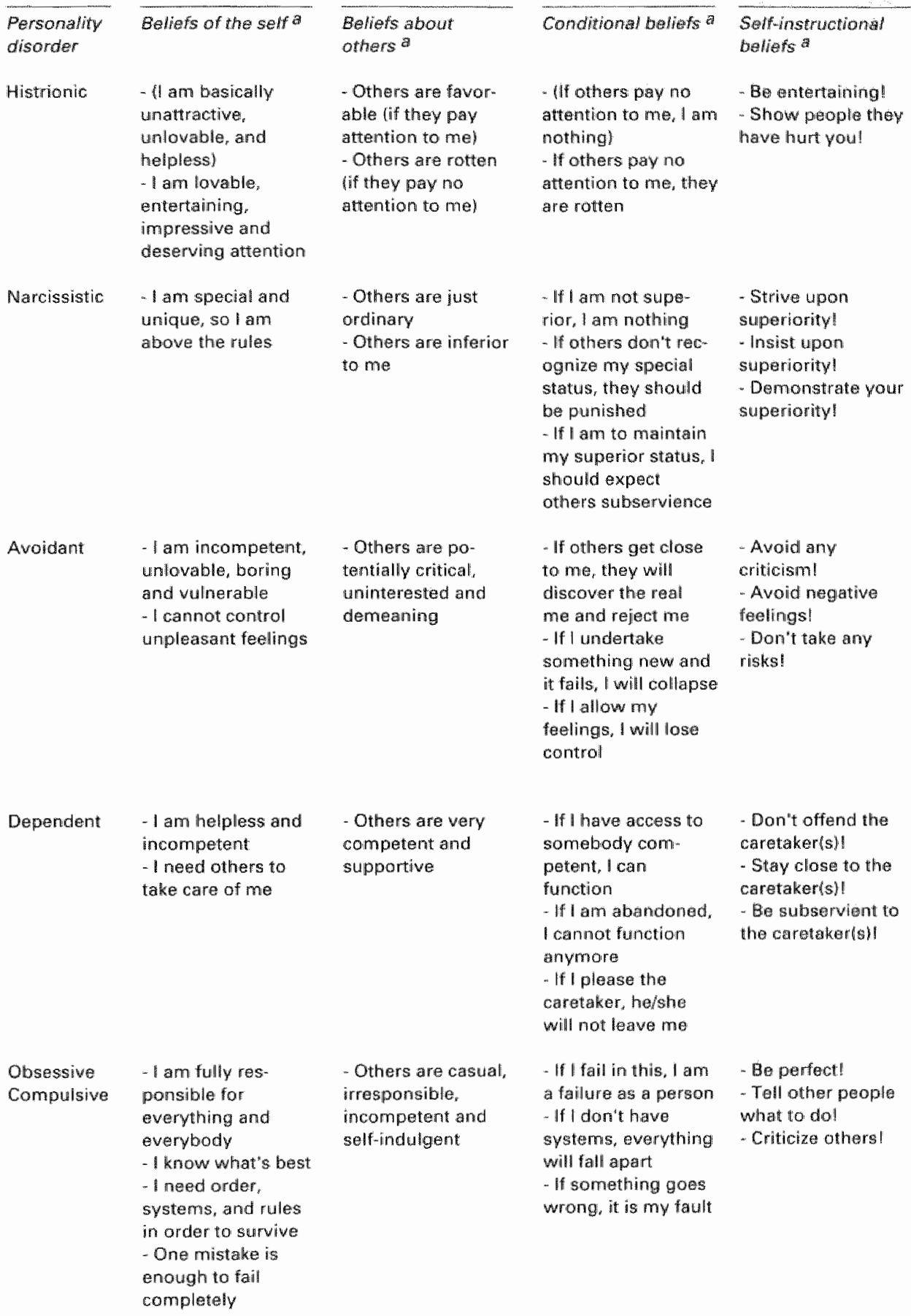


Table 2 (continued)

\begin{tabular}{|c|c|c|c|c|}
\hline & & & & \\
\hline $\begin{array}{l}\text { Personality } \\
\text { disorder }\end{array}$ & Belief's of the selt & $\begin{array}{l}\text { Befiefs about } \\
\text { others }\end{array}$ & Conditional beliefs a & $\begin{array}{l}\text { Selfinstructional } \\
\text { beliefs }{ }^{a}\end{array}$ \\
\hline Despressive & $\begin{array}{l}\text { - I am a fallure and } \\
\text { will stay a failure } \\
\text { - I do not deserve no } \\
\text { be happy }\end{array}$ & $\begin{array}{l}\text { - Others are too } \\
\text { optimistic: }\end{array}$ & $\begin{array}{l}\text {-If have hopes, } \\
\text { will be disappointed } \\
\text { If ofhers are pos- } \\
\text { itwe ablot me of the } \\
\text { future, they have } \\
\text { overlooked } \\
\text { something }\end{array}$ & - Have no hopes! \\
\hline $\begin{array}{l}\text { Pagsive } \\
\text { aggressive }\end{array}$ & $\begin{array}{l}\text { - } 1 \text { am vumerable to } \\
\text { encroachment by } \\
\text { others } \\
\text { - I need authority to } \\
\text { mature and support } \\
\text { me }\end{array}$ & $\begin{array}{l}\text { - Others are } \\
\text { intrusive, de. } \\
\text { manding. } \\
\text { cuntrolling and } \\
\text { dominating } \\
\text { - Others are } \\
\text { approwing. } \\
\text { accepting and } \\
\text { caring }\end{array}$ & $\begin{array}{l}\text { - If I follow the rules, } \\
\text { I will lose my } \\
\text { freedom of action } \\
\text { - If I refuse to follow } \\
\text { the rules, authority } \\
\text { will not support me }\end{array}$ & $\begin{array}{l}\text { - Postpone expected } \\
\text { action! } \\
\text { - Scrub round nules! } \\
\text { - Just piretent to } \\
\text { comply! }\end{array}$ \\
\hline
\end{tabular}

a The cognitive profiles are based on the cognittwe model of Beck and colleagues (Becket al., 1990) and on the Personalizy Disorder Belief Qwestionnaire (PDBQ; Dreessen, Amtz, \& Weertman, 1986)

In an Axis I therapy, PD schemas are not the focus of treatment, but nevertheless they may act upon the relationship with the therapist and the therapy process. Emotional and behavioral responses that limit or advance therapy may be explained from operating PD schemas. For instance, patients who fail to do homework assignments, may have one or more of the following PD-assumptions: 'It is better not to do anything, than to try something that might faill', 'I should avoid unpleasant feelings, because they will escalate and get out of control', or 'Efforts to change something are useless'. On the contrary, patients who do home-work assignments properly, may have one or more of the following assumptions: 'It is important to do a perfect job on everything" or "I must be subservient to others in order to maintain their good will'.

Few empirical studies have addressed the validation of the cognitive theory of DSM PDS. Clearly, the theory needs to be further tested. A way of testing the theory is to demonstrate convergent validity (e.g. the association between two tests or constructs that in theory should be associated) and discriminant validity (e.g. the absence of an association between two tests or constructs that are purportedly independent) (Widiger, 1993). First, it can be studied whether DSM PDS are specifically associated with schema-congrtent biases in the information processing. So far, no such study has been carried out (section 5.2 of the present thesis offers a first attempt). Second, standardized assessment instruments need to be developed which measure amount of credence attached to the typical PD beliefs (instead of deriving idiosyncratic beliefs from thinking affect, and behavior, the person is asked to rate the credibility on a list with pre-formulated beliefs). Thereupon, it can be studied whether each of the DSM PDS is specifically associated with its own typical set of beliefs. To date, only three instruments have been designed to measure maladaptive personality schemas. Beck developed the Belief Questionnaire (BQ, Beck, 1990; for psychometric properties see Trull, Goodwin, Schopp, Hillenbrand, 
\& Schuster, 1993), which attempts to measure beliefs hypothesized to be typical for nine DSM-II-R PDS. Young developed the Schema Questionnaire (SQ, Young, 1990; for psychometric properties see Schmid, Joiner, Young, \& "Telch, 1995), which attempts to measure 16 early maladaptive schemas that are theoretically related to personality disorders, but not isomorphic with the DSM PDS. In section 5.1 of the present thesis, the third questionnaire is presented, the Personality Disorder Belief Questionnaire (PDBQ, Dreessen, Arntz, \& Weertman, 1996), designed to measure PD beliefs. The 12 subscales correspond with the 12 DSM-IVDS. The BQ (Beck, 1990) and the PDBQ (Dreessen et al., 1996) overlap partially.

\section{Main research questions addressed in this thesis ${ }^{2}$}

In the preceding, relevant issues in the field of PDs were touched on and the main research questions addressed in this thesis were mentioned indirectly by referring to the studies in the sections concenned. Below the main research questions addressed in this thesis are outhed explicitly. For a more detailed background of the hypotheses, the reader is referred to the introduction of the separate studies.

1 What is the test-retest interrater reliability of the Structured Clinical Intervicw for DSM-III-R Personality Disorders (SCID-M3), both for categorical and dimensional PD variables in a group of independent raters (experienced clinicians) interviewing outpatients (referved for cognitive behavior therapy, mostly for anxiety disorders)? (Section 2.1 of this thesis).

The SCID-II (Spitzer et al., 1990) is one of the most well-known and frequently used semistructured interviews ${ }^{4}$ designed to assess DSM PDS (Zimmerman, 1994). Jointinterview interrater reliabilities of the SCID-II have already been found to be good in various groups of raters. The short-interval test-retest approach is a more thorough examination of reliability, taking into account rater variance in the elicitation and interpretation of information and patient variance in providing information.

2 Are patients with florid Axis I disorders a valid source of informmion in the assessment of PDS by the SCID-11, or should we rely more on informant reports? (Section 2.2 of this thesis).

Poor concordance between patients and informants based diagnoses has already been demonstrated in prior studies. However, such concondance has never been studied using the SCID-II to assess PD diagnoses. In the absence of a golden standard, good agreement between patient and informant SCID -1 diagnoses is indicative for the validity of patient reports, using the SCID-II. In case of poor patient-informant conoordance, a third source of information, namely the therapist who is asked to give personality ratings, is used in an attempt to determine which of the two sources is more valid (patients or informants).

3a Do (specific) pDs have a negative impact on therapy outcome in the individually tailored cognitive and behavional treatment of various anxiety disorders, lumped together as a group (Section 3.1 of this thesis). 
3 b Do (specific) pDs have a negative impact on therapy outcome in short term cognitue and behavioral treatment protocols for panic disorder? (Section 3.1 of this thesis).

3c. Do (spectic) pos have a negative impact on therapy outcome in short-term cognitioe and behavioral tratnent protocols for obsessive compulsioe disorder? (Section 3.2 of this thesis).

Both the impact of categorical and dimensional PD diagnoses are studied. In the individually tailored treatment, number of sessions is unlimited and the therapist is free to choose any combination of cognitive and behavional techniques. In the treatment protocols, on the other hand, number of sessions and cognitive behavioral treatment techniques are standardized. In answering the above questions, methodology of the studies is improved compared to prior studies on this issue: PDs are assessed before start of the treatment by an independent rater using the Scro-II. Treatment outcome is defined as change with respect to the pretest scores.

4 Do PDs complicate the therapy process in cogmitive and behavioral treatment of various antety disorders?

Do therapists experiance that they can overcome the complication caused by conconitant pDs? (Section 3.3 of this thesis).

PDs were assessed by an independent rater using the SCID-II. Also, the therapist gave PD-ratings. SCID-II- based diagnoses and therapist-based diagnoses were compared to answers on a self-constructed questionnaire measuring $A$ xis I therapy process variables. To our knowledge, the impact of PDs on the Axis I therapy process has never been studied before.

5 Is the DSM-IIT-R avoidant PD characterized by so-called avoidant schemas and do these schemas direct the processing of information resulting in schema-congruent biases? (Section 5.2 of this thesis).

Cognitive theory of PDS assumes that PDS are characterized by a typical set of schemas that result in schema-congruent information processing biases. Although of great clinical interest, little empirical work has addressed the validation of the cognitive theory and much more research is needed. The present thesis attempts to contribute to this major endeavor by presenting the development of an experinental task (the pragmatic inference task) and an initial attempt in the validation of the cognitive model for the avoidant PD. DSM-III-R avoidant personality pathology is measured by the SCID-Personality Questionnaire. So-called avoidant schemas are measured by the Personality Disorder Belief Questionnaire (Dreessen et al. 1996, see section 5.1 of the this thesis). 


\section{notes}

1. As was pointed out by Shea $(1992, p, 377)$; 'It is important to make a distinction between validity of personality disorder constructs and validity of personality disonder assessment. The former refers to evidence supporting a theoretical entity, whereas the latter refers to the ability of measurement tools to assess such construct. The field of personality disorders is struggling with both these issues simultaneously"

2 PDs in all studies presented in this thesis were diagnosed according to the DSM-III-R (APA, 1987). Although the DSM-IV (APA, 1994) was published in 1994, the adaptation of standardized assessment instruments for Axis I and Axis II disorders and the subsequent tramslation into Dutch language were finished not untill 1997.

3 The SCID-II interview is the instrumert of choice in all clinical studies presented in this thesis. This interview was choosen because it is one of the most well-knowrin and frequently used PD interviews and becalse it was already used in the Community Mental Health Center at Maastricht. All patients were recruited from this center:

4 The other well-known and frequently used standardized interviews are (1) The Structured Interview for DSM-IIIl Personality Disorders (SIDP; Pfoll et al., 1995), and (2) The Personality Disorders Examination (PDE; Loranger et al., 1994). 


\section{Assessment of personality disorders: the Structured Clinical Interview for DSM-III-R Personality Disorders (SCID-II)}

In Chapter 2, two studies are presented on the assessment of personality disorders by the Structured Clinical Interview for DSM-III-R personality disorders (SCID-II), a frequently used and well-known instrument. The first study concerns the shortinterval test-retest interrater reliability (section 2.1) and in the second study convergent validity is tested by comparing patient based and informant based diagnoses (section 2.2). 


\section{Short-interval test-retest interrater reliability of the Structured Clinical Interview for DSM-III-R Personality Disorders (SCID-II) in outpatients ${ }^{1}$}

\section{Summary}

The short-interval test-retest interrater neliability of the Structured Clinical Interview for DSM-H-R Personality Disorders (SCID H) was studied in a psychotherapy outpatient group whose main complaint was nostly an Axis Ianxiety disorder. Using a test-retest approach to assess interrater reliability, three sources of variance were taken into account (rater yariance in the elicitation and interpretation of information and patient variance across interviews). Base rate requirements were established before calculating reliability coefficients. On the whole, interrater agreement on the Scm-II was found to be satisfactory, except for the histrionic personality traits. This is the first study that has estmated short-interval test-retest interrater reliability of the SCID-I in outpatients, and also the first that has studied single SCID-II traits and dimensional diagnoses. The results found suppont the use of the SCID-II as a diagnostic instrument for clinical and research purposes.

\section{Introduction}

Diagnostic reliability, a prerequisite for accurate assessment, is not easily achieved in the assessment of personality disorders (PDS). The rater is burdened with making difficult decisions such as whether the pattern is pervasive and persistent enough across time and situations, which complicates diagnostic agreement. Several authors have indicated their preference for (semi)structured interviews above selfreport questionnaires and unstructured interviews in diagnosing PDs (e.g. Dreessen \& Arntz, 1998-a; Zimmerman, 1994). Self-report questionnaires are often believed to have more utility as a screening instrument than as a diagnostic instrument (e.g. Loranger, 1992; Ouimette \& Klein, 1995).

The Structured Clinical Interview for DSM-III-R Personality Disorders (SCIDry(Spitzer, Williams, Gibbon, \& First, 1990) is a frequently used instrument by researchers and clinicians. The SCID-II is semistructured and covers the DSM PDS (American Psychiatric Association, 1987). In order to conduct the interview, clinical experience and extensive training are required. Joint-interview interrater reliability studies of the SCD-II have shown good reliability coefficients across studies (Amtz et al., 1992-b; Brooks, Baltazar, McDowell, Munjack, \& Bruns, 1992; Fydrich, Schmitz, Hennch, \& Bodem, 1996; Renneberg, Chambless, Dowdall, Fauerbach, 
\& Gracely, 1992; Stanley, Tumer, \& Borden, 1990; Wonderlich, Swift, Slotnick, \& Goodman, 1990).

The short-interval test-retest approach is a more thorough examination of reliability. In this approach, two raters separately interview the same patient. In this way, three sources of variance are being tested:

1 rater variance in the elicitation of information;

2 rater wariance in the interpretation of information;

3 patient variance in providing information across interviews.

In the joint-interview approach, only rater variance in the interpretation of information is being tested.

In semistructured interviews, such as the SCD-II, the rater is allowed to ask further questions following on from the structured questions, and to discuss the evidence with the patient (e.g. to check whether the traits are consistent across time and situations and whether they have caused problems). Consequently, the amount and quality of the elicited information are rater dependent. Compared to joint-interview reliabilities, short-interval test-retest reliabilities are, therefore, more generalizable to the actual practice, in which different raters conduct the interview.

Patient variance in providing information in semistructured interviews is not only rater dependent, but also time dependent. A reliable interview for PDs is one in which a patient's responses and behavior would be comparable if one interview-er were substituted for another and if the patient were interviewed today or in a few weeks time. In conclusion, rater variance in the elicitation of information and patient variance in providing information across interviews are very important issues to be tested with semistructured interviews.

To our knowledge, only three studies have examined the short-interval testretest interrater reliability of the SCID-II or parts of it (First et al., 1995-b; Hennch, Lauer, \& Fydrich, 1994; Malow, West, Williams \& Sutker, 1989). Overall, test-retest relliabilities of the SCID-II varied from poor to good and were less consistent than the joint-interview reliabilities of the SCID-II.

In this article, we describe a study on the short-interval test-retest reliability of a Dutch version of the SCID-II. Whereas earlier studies used inpatients, or a mixture of inpatient and outpatients, we used only outpatients in our study. In contrast to the First et al. (1995-b) study, we assessed all SCID-II criteria, without initial screening by means of the SCID-Personality Questionnaire. Furthermore, this is the first study that has evaluated single SCID-II traits and dimensional diagnoses. In the assessment of PDS, dimensional diagnoses are increasingly preferred to categorical diagnoses (e.g. Widiger, 1992).

\section{Mèthod}

\section{Patients}

The participants were 43 outpatients who were referred to the "Academic Section Behavior Therapy' of the Community Mental Health Center Maastricht. Following everyday practice at our section, an intaker administered an unstructured clinical interview, followed by the Structured Clinical Interview for DSM-III-R (SCID: Spitzer, 
Williams \& Gibbon, 1987; Dutch translation: Koster van Groos, 1985; Arntz, Bögels, \& Hoekstra, 1992-a) to assess Axis I disorders. All but two of the patients had one $(n=36)$ or more $(n=5)$ anxiety disorders. Furthermore, three patients had a depressive disorder, three had an eating disorder and three had a somatoform disorder. The unstructured clinical interview also revealed that one patient had an identity disorder, that one had a stuttering disorder and that four had a VCode problem. All patients were referred for cognitive and/or behavior therapy for Axis I disorders.

There were 20 men and 23 women. The mean age was 33 years (range 19-60), 61 per cent were married or living together with a partner.

\section{Raters}

There were six raters: two clinical psychologists (A.A. \& S.B.), and four mental health scientists (L.D., M.vR, W.K., \& M.H.; degree is comparable to clinical psychollogy) all of whom were experienced in diagnosing and treating psychiatric patients. Five of them practiced as therapists in the Community Mentall Health Center Maastricht, and were either officially registered (cognitive-behavioral) psychotherapists or in training to become such therapists. All raters were extensively trained in administering the SCID-II by doing joint-interviews with experienced SCID-II interviewers [from the Amtz et al. (1992-b) study], in which they were first and second rater by turns. Afterwards they discussed uncertainties and difficulties, and feedback was given to improve their elicitation and interpretation of information.

Each rater acted approximately the same number of times as first rater and as second rater. In a subsample of 24 patients, four raters (A.A., L.D., M.vR., W.K.) were paired with each other to act together twice as first rater and twice as second rater, in order to control for effects resulting from rater combinations. Several raters participated in the study in order to increase generalization of the results. A fifth rater started to follow this procedure of combinations, but completed only seven of all possible combinations. In the remaining $11 \operatorname{cases}^{2}$ (which were added to increase the sample size), two raters (L.D., M.H.) were alternately first and second rater.

\section{Instrument}

The (semi)Structured Clinical Interview for DSM-III-R Personality Disorders (SCIDII)(Spitzer et al., 1990; for a full description see First, Spitzer, Gibbon, \& Williams, 1995-a; Dutch translation: PC Bloemendaal, 1991) was used for the assessment of personality pathology. The original SCID-II does not include questions about the sadistic PD. Arntz et al. (1992-b) formulated standard questions for the DSM-III-R sadistic traits. In clinical practice, the SCID-Personality Questionnaire can be used as a screening tool to shorten the time that it takes the clinician to administer the SCID-II interview. Because this procedure might inflate reliability artificially, interviewers in our study were instructed to discuss all SCID-nI traits with the patient. Traits were rated as threshold or true (3), as subthreshold (2) or as absent (1). As in the DSM-HII-R (APA, 1987), the trait must be stable over time and situations, and not be merely present in the period of Axis I disorders or in particular situations. The mean time to complete the first interview was 107 minutes $(S D=24)$ and the mean 
time to complete the second interview was 104 minutes $(S D=24)$, which indicates that the criteria were thoroughly discussed. There was no difference between the means (paired t-test: $t(41)=0.61, p=.55$; data was missing for one interview).

\section{Procedure}

Following the unstructured clinical interview and the SCID for Axis I disorders, written informed consent was obtained for participation in the present study. Next, the SCID-II interview was administered by the first rater. The second rater attempted to reinterview the patient within one to four weeks of the first interview being administered and also before therapy started. For various reasons, this was not achieved in all cases (e.g. some patients cancelled appointments, sometimes the waitinglist for starting therapy was very short). In 11 cases, therapy had already started, when the second interview took place. The mean number of days between administration of the first and second interview was $35(S D=34)$. The patient and the second rater were blind to the results obtained from the first interview.

In 24 cases, the intaker and the first rater of the SCID-II were the same person. Before administering the SCID-II, both the first and the second rater were instructed to study the patient file, including intake report, Axis I SCID evaluation and background information. This information is indispensable for the evaluation of the responses to the SCID-II interview questions (First et al., 1995-a).

\section{Statistical Analyses}

Short-interval test-retest reliability was calculated for several categorical and dimensional PD variables. The categorical PD variables were (1) presence of one or more full-blown PDS, regardless of type; (2) presence of one or more subthreshold or full-blown PDS, regardless of type; (3) presence of specific PDs. The dimensional PD variables were (1) number of traits for specific PDs (traitscore); (2) SCID-II sumscore of specific PDs. Sumscores of specific PDs were obtained by adding up all the scores that were given on the traits concerned $(1,2$, or 3$)$; (3) total number of SCID-II traits; (4) total sumscore of the SCID-II; and (5) scores on single SCID-II traits $(1,2$ or 3$)$.

Cohen's kappa (Cohen, 1960) was used for estimating agreement on categorical PD variables. The Intraclass Correlation Coefficient (random raters; ICC; Fleiss, 1986) was used for estimating agreement on dimensional PD variables. Generally, ICC and kappa values larger than 0.75 are taken to indicate excellent agreement, values below 0.40 ind icate poor agreement ${ }_{r}$ and values in between indicate fair to good agreement (Fleiss, 1981; Landis \& Koch, 1977).

Diagnostic reliability is influenced by the illness base rate: a few diagnostic disagreements have a more pronounced effect on reliability when the base rate is either high or low (Zimmerman, 1994, p. 226). Therefore, the following illness base rate requirements were made. Kappa was calculated only if at least five cases were diagnosed with a PD by at least one rater. ICCs for trait- and sumscores were calculated only if at least five cases were diagnosed with two or more traits of that particular PO by at least one rater. ICCS for single SCID-II traits were calculated only if the trait was fully diagnosed in at least five cases by at least one rater. 


\section{Results}

The frequency of patients with full-blown and subthreshold diagnoses are presented in Table 1. Kappa for the presence of one or more full-blown PDs was 0.53 , which indicates fair to good agreement (observed agreement $=84 \%$ ). When patients with only subthreshold diagnoses were also included in the PD group, kappa was 0.56 (observed agreement $=79 \%$ ). With regard to the specific PDs, kappa could be caliculated only for the avoidant $\mathrm{PD}$, and it was found to be good (kappa $=0.73)^{3}$. Observed agreement for the specific PDs ranged from $93 \%$ to $100 \%$.

TABLE 1

Frequency rates of personality disorders and mean number of traits according to the first and the secand SCID-II

Frequency af patients with fellilloblown a PD (with subthreshold b PD)

Personafity disorder
Avoidant
Dependent
Obsessive compulsive
Passive aggressive
Self-defeating
Paranoid
Schizotypal
Schizaid
Histrionic
Narcissistic
Borderline
Antisocial B
Antisocial C
Sadistic
One or more

$\mathrm{PD}=$ personality disorder

Anthisocilall $B=$ antisocial behavior before age 15

Antisociall C - antisocial behavior simce the age of 15

a Full-blown PD diagnoses were made if the patient futflled the minim num numer of traits required by the DSM-III-R (APA, 1987)

b Subthreshold PD diagnoses were made if the patient fulfilled the minimum number of traits minus one required by the DSM-III-R (APA, 1987)
Mean number of traits trange)

\begin{tabular}{ll} 
First rest & Second test \\
$1.5(11-5)$ & $1.3(0-5)$ \\
$1.4(0-6)$ & $1.2(0-5)$ \\
$1.2(0-7)$ & $0.9(0-5)$ \\
$0.5(0-4)$ & $0.6(0-4)$ \\
$0.4(0-3)$ & $0.6(0-4)$ \\
$1.0(0-5)$ & $0.9(0-5)$ \\
$1.1(0-4)$ & $1.0(0-4)$ \\
$0.4(0-2)$ & $0.4(0-2)$ \\
$0.5(0-4)$ & $0.3(0-3)$ \\
$0.4(0-3)$ & $0.3(0-3)$ \\
$0.7(0-5)$ & $0.5(0-4)$ \\
$0.4(0-4)$ & $0.3(0-3)$ \\
$0.2(0-2)$ & $0.2(0-2)$ \\
$0.02(0-1)$ & $0(0)$ \\
- & - \\
\hline
\end{tabular}

The mean traitscores of the specific PD categrories are presented in Table 1. ICCs for the trait- and sumscores were calculated for all specific PD categories, except for the schizoid, the narcissistic, the antisocial $C$ and the sadistic trait- and sumscores. All ICC values indicated fair to good or excellent agreement (range from 0.49 to 0.86 ; Table 2), except for the histrionic trait- and sumscore, of which the ICc values were unsatisfactory $(<0.40$; Table 2$)$. The Iccs for the total number of full-blown PDs and the total number of subthreshold and full-blown PDs taken together indicated good agreement ( 0.67 and 0.71 , respectively). The same held for the ICCs for the total number of traits and the sumscore of the SCID-II $(0.72$ and 0.73 , respectively). 
The original SCID-II contains 108 different traits (double traits excluded) and we added the eight DSM-M-R sadistic traits. The ICC could be calculated for 35 traits and 27 reached an ICC value larger than 0.40 , indicating fair to good agreement between the first and the second SCID-II test (Table 3).

\section{TABLE 2}

Short-interval test-retest interrater reliabilities (ICCs) of trait-and sumscores of specific personality disorders

\begin{tabular}{ll}
\multicolumn{2}{c}{ ICC $a$} \\
\cline { 1 - 1 } Tratscore & Sumscore \\
0.80 & 0.86 \\
0.49 & 0.64 \\
0.75 & 0.84 \\
0.62 & 0.60 \\
0.53 & 0.53 \\
0.66 & 0.63 \\
0.59 & 0.71 \\
- & - \\
0.24 & 0.36 \\
-0.72 & - \\
0.75 & 0.75 \\
- & 0.70 \\
& - \\
\hline
\end{tabular}

$\mathbb{H C C}=$ Intraclass Correlation Coefficient (random raters)

Antisocial $B=$ antisocial behavior before age 15

Antisocial $C=$ antisocial behavior since the age of 15

a ICCs were calculated only if at leasit five cases were diagnosed with

two or more traits of that particular personallity disorder by at least one rater

b 10 or more cases had two or more traits of that particular personality disorder diagnosed by at least one rater 
TABLE 3

Short-interval test-retest interrater reliabilities (ICCs) of the single traits of the SCID-Al

Personality disordar

Avoidant 17 traitsil

Dependent $(9$ traits)

Obsessive compulsive (9 traits)

Passiwe aggressiwe (9 traits)

Selfudefeating (8 trails)

Paranoid (7 traits)

Schizotypal 19 traits!

\section{Schizoid (7 traits)}

Histrionic ( 8 traits)

Narcissistic (9 tratuts)

Borderline (s trails)

Antisociall B 12 rraits

Antisocial C (10) traits)

7. Reckless

1. Easily hurt by criticism

2. No intimate friends

3. Awoids personall contact with people

4. Awoids sociall-wallk actiwities

5. Reticent in social situations

6. Fears of being embartassed

3. Always agrees

5. Does inpleasant jobs in order to be liked

6. Avoids being alone

7. Feels helpless when close relationships end

8. Preoccupied with fears of being abandoned

9. (see avoidant personality disorder trait 1)

3. Insisits that others do things his or her way

4. Excessively devoted to work

5. Indecisive

6. Overconscientious about matters of morality, etc

7. Restricted expression of affection

9. Saves worthless things

2. Irritable when something umpleasant is asked

5. 'Forgets obligations

6. Feels underappreciated

1. Chooses disappointments, failures etc.

2. Rejects help

1. Expects to be exploited

2. Questions the loyalty of friendls or colleagues.

3. Sees hidden dangers

4. Unforgiving

5. Trusts no one

6. Easilly salighted annd angry

2. Excessive social anxiety

4. Unusual percepiual experiences

6. (see avoidant personality disorder trait 2)

9. (at least one of the first five paranoid traits)

6. Isee avoidant personality disorder trailt 2 )

1. Consitantly demands approval

3. Overly concerned with appearance

1. Angry after criticism

0.54

3. Affective instability

0.48

0.43

0.77

Sadistic 18 traits

ICC = Intraclass Correlation Coefficient (random raters)

Antisocial $B=$ antisocial behavior before age 15

Antisocial $C=a n t i s o c i a l$ behavior since the age of 15

a ICC for single criteria was calculated only if at least 5 cases were rated positively $\{3$ by at least one rater

b 10 or more cases were rated positively $(=3)$ by at least one rater 


\section{Discussion}

In the present study, short-interval test-retest interrater reliability of the SCID-II was studied in a psychotherapy outpatient group. The results can be summarized as 6ollows:

1. Agreement on the presence of one or more PDs (subthreshold diagnoses included or not) was fair;

2 Agreement on the presence of the avoidant PD was good. Agreement on the other specific pos could not be estimated;

3 Agreement on the trait- and sumscores of specific $\mathrm{PD}$ categories ranged from fair to excellent, except for the histrionic PD, on which raters showed poor agreement. Agreement on the schizoid, narcissistic, antisocial $C$ and sadistic trait-and sumscore could not be estimated;

4 Agreement on the majority of the single traits that could be tested, was satisfactory.

These results support the use of the SCID II as a diagnostic instrument for clinical and research purposes.

Short-interval test-retest interrater reliability of the SCID-n has not been studied before as far as the single SCID-II criteria are concerned. In the present study, agreement on the single SCDD-II traits was not as high and consistent as agreement on trait- and sumscores of specific PDs. This could mean that raters have some difficulty agreeing on specific criteria, whereas they are quite capable of achieving good agreement with respect to dimensional trait-and sumscores, that are less specific than single traits. However, before this can be definitely concluded the above results would have to be replicated.

Poor agreement on the histrionic trait-and sumscores in our study may be due to the relatively low frequency of histrionic traits. In the first set of interviews, six patients had two or more histrionic traits. In the second set of interviews, only three patients had two or more traits. When the frequency is low, a few diagnostic disagreements have a pronounced effect on reliability. Furthermore, we checked whether the poor results were caused by particular traits. We found that exclusion of each histrionic trait from the analyses one at a time did not result in a substantial increase in the $1 \mathrm{CCS}$ (ICCs $\leq 0.43$ ). It remains to be shown whether a larger frequency of histrionic traits results in higher interrater agreement. We also considered the possibility that the items of the histrionic PD were characterized by more subthreshold ratings ( 2 ratings instead of 1 or 3 ) compared to the other PD categories. Less obvious cases are more difficult to agree on than obvious cases. However, this explanation was not proven plausible. The proportion of subthreshold ratings with the histrionic traits (= number of 2 ratings as a per cent of number of 2 and 3 ratings $=45 \%$ ) was not deviant from the proportion of subthreshold ratings with other PD categories $(M=43 \%)$.

In the following paragraphs, several noteworthy aspects of the present study will be discussed. First, to our knowledge, this is the first study in which shortinterval test-retest interrater reliability of the SCID-II has been estimated by using dimensional scales of measurement (trait- and sumscores of specific PD categories). In the literature on PDS, several authors favor the dimensional classification of PDS 
above the categorical classification of PDs. Thresholds appear to be largely arbitrary and reliability and validity have repeatedly been found to be better with the dimensional ratings than with the categorical diagnoses (see Widiger, 1992, p. 290,293 ). The results of the present study also indicate that the dimensional classification of PDs is to be preferred above the categorical classification. First, using a dimensional scale of measurement, higher reliabilities were found in the present study than in previous studies in which categorical diagnoses were evaluated (First et al., 1995-b; Hennch et al., 1994). Second, we found an increase in the reliability coefficients when evaluating sumscores instead of traitscores of specific PDS. Sumscores are more dimensional than traitscores, because they include subthreshold ratings on single traits.

A second noteworthy aspect of the present study is that it is the first to evaluate short-interval test-retest interrater reliability of the SCID-II in outpatients. Earlier studies on the short-interval test-retest interrater reliability of the SCID-II used inpatients or a mixture of inpatients and outpatients. Outpatients can be expected to have less severe and obvious personality traits. This could complicate diagnostic decision making with respect to personality pathology and affect reliability negatively. The present study provides evidence against this hypothesis by finding good test-retest reliabilities of the SCID -II in an outpatient sample.

A third aspect worth mentioning is that we ruled out any artificial inflation of reliability by not using the SCID-Personality Questionnaire as an initial screening. as was done in the First et al. (1995-b) study. All SCID-II criteria were evaluated independently by both raters.

It is also worth noting that there were no differences between the first and the second set of interviews with respect to the mean time to complete the interview (see paragraph about Instrument) and with respect to the mean number of personality traits rated as present (paired t-test: $t(42)=1.04, p=.30$ ). Moreover, the first and the second set of interviews did not differ with respect to the total number of PDs (subthreshold diagnoses included, see Table 1; McNemar test: $p=$ .51). These findings are of interest because they are not consistent with the criticism on the test-retest approach made by Loranger (1992). He stated that patients might be less motivated and bored during the repeat interview. As a consequence, patients may want to reduce interview time by giving negative answers, which are less likely to be followed by further probing. In our study we found no such evidence of boredom and decreased motivation among the patients.

As a critical comment on the present study it should be noted that the sample size was small and the frequency rate of some PD variables was very low. Consequently, some specific PD categories were excluded from all analyses (schizoid, narcissistic, antisocial $C$ and sadistic) and kappa could only be calculated for one specific PD. Although the dimensional model is increasingly favored, the categorical approach is still customary in clinical practice, and therefore kappas are of interest. Furthermore, it should also be noted that for those PD variables with a frequency rate close to the minimum required, a few disagreements can still have a marked impact on the overall reliability. In other words, base rate requirements do not completely solve the problem of establishing diagnostic reliability in homogeneous samples. Future studies would better select the patients to enhance the frequency of specific PDs. In addition, with small sample sizes, 
reliability coefficients have a large standard error of measurement, and thus are less stable (Fleiss, 1981). Results based on a small sample should, therefore, be interpreted more cautiously than results from larger samples (Zimmerman, 1994) and replication is needed.

It is important to realize that sample characteristics (e.g. prevalence and severity of PDs) and rater characteristics (e.g. clinical experience, motivation, personal style) may have a considerable impact on reliability in a particular study (see also First et al., 1995-b). Consequently, results of reliability studies with equal experimental designs may differ (as is the case with the studies on short-interval test-retest interrater reliability of the SCID-II) and should not be generalized too easily to other types of patients and raters than those being studied.

In conclusion, the present study showed satisfactory short-interval test-retest interrater reliabilities for the SCID-II in psychotherapy outpatients. In our opinion, the test-retest approach is a better way to estimate the interrater reliability of semistructured interviews for PDS than the joint-interview approach, because it takes into account rater variance in the elicitation of information (in addition to rater variance in the interpretation of information). Moreover, the test-retest approach takes into account patient variance in providing information across time, which is also an important factor in studying reliability of instruments for the assessment of personality pathology.

\section{notes}

1 Published as: Dreessen, L. Arntz, A. (1998-b). Short-interwal test-retestinterrater reliability of the Structured Clinical Interview for DSM-III-R Personality Disorders (SCID-ID) in outpatients. Journal of Personndity Disorders, $12,138-148$.

2 Data of these 11 cases was also used as and indication of shor-interwal test-retest reliability beween the two raters who participated in astudy on patient-intormant concordance on the SCID-Jl (Dreessen, Hildebrand, A Arnt $z, 1998$-a).

3 Comorbidity in patients with avoidant personality disorder, diagnosed by at least one rater; with Axis I generalized sacial phobia is $38 \%$ ( 3 out of 8 ). 


\title{
Patient-informant concordance on the Structured Clinical Interview for DSM-III-R Personality Disorders (SCID-II)
}

\begin{abstract}
Summary
In the assessment of personality disorders, patient reports can be questioned for several reasons, such as lack of insight shame and acute psychiatric state. High concordance between patient based and informant based diagnoses would be an indication of the validity of patient reports (convergent validity). The present study examined the concordance between 42 psychotherapy outpatients and their informants (intimates) on the Structured Clinical Interview for DSM-III-R Personality Disorders (SCD-II). Similar to prior studies, low or only modest levels of agreement were found. In comparison with evaluations of the personality of the patient by the therapist, patient interviews seemed to be more valid than informant interviews. Furthermore, couples with high intensity and intimacy in the contact generally showed higher agreement than couples with low intensity and intimacy. In conclusion, the data slightly suggest that patient reports are more valid than informant reports. However, the lack of a golden standard forces us to find more evidence before concluding that patient reports on personality result in valid diagnoses.
\end{abstract}

\section{Introduction}

In the assessment of personality disorders (PDS) patients are usually the primary source of information. The validity of patient reports can be questioned for reasons such as lack of insight and underreporting due to shame. Personality traits are usually egosyntonic owing to which the patient may not teel out of range of normality. Even if the patient is aware of the trait, he or she might be too ashamed to report socially undesirable behavior, such as egocentricity or exploitativeness.

Moreover, the acute psychiatric state of the patient might cause biases in patient reports. Interviewers can be trained to separate Axis I and Axis II pathology, but it is still the patient who might provide ambiguous information on this matter. It is also well-known that a state of depression or anxiety can bias the memory of past experiences (Williams, Watts, Macleod, \& Mathews, 1988).

The main difficulty in testing the validity of patient reports on personality pathology is the lack of a golden standard. Nevertheless, convergent validity can be tested by comparing patient based and informant based diagnoses (Widiger, 
1993). Report by informants is bellieved to be less biased by the problems mentioned above than report by patients. Nevertheless, report by informants is not the golden standard. It can be questioned for reasons such as lack of detailed knowledge about the past of the patient. In the absence of a golden standard, good agreement between patient and informant interviews is indicative for the validity of patient reports. Poor agreement, on the other hand, leaves unanswered the question of whether patients are a valid source of information or not.

Five prior studies reported poor, or only modest patient-informant concordance (Dowson, 1992; Riso, Klein, Anderson, Ouimette, \& Lizardi, 1994; Steketee, 1990; Tyrer, Alexander, Cicchetti, Cohen, \& Remmington, 1979; Zimmerman, Pfohl, Coryell, Stangl, \& Corenthal, 1988).

In contrast to these prior studies, we used a different instrument for the assessment of PDS (the Structured Clinical Interview for DSM-III-R Personality Disorders, see Method). As another important difference, we attempted to determine which source of information is more valid by comparing the diagnoses of the patient and informant interviews to the personality diagnoses made by the therapist.

Each rater administered as many interviews with patients as with informants. This design is preferable, because interrater agreement is never perfect and rater biases should be controlled for as much as possible.

Besides diagnostic agreement for various PD variables, we also examined differences in prevalences between the patient and informant interviews, and factors that might be related to concordance (e.g. quality of the relationship between patient and informant).

\section{Method}

\section{Patients}

During the investigation, 66 patients were referred to the "Academic Section Behavior Therapy' of the Community Mental Health Center for outpatients at Maastricht. They were all asked to participate in the study. However, six patients were not able to nominate an intimate according to the criteria of the present study (see Informants below). Eight patients refused to participate. Ten other patients refused to let an informant participate or the informant refused to participate. The finall sample consisted of 42 patients who were referred to receive cognitive and / or behavioral treatment for anxiety disorders $(n=35)$, eating disorders $(n=4)$, somatoform disorders $(n=1)$ or Axis 11 disorders $(n=2)$. The Axis I disorders were assessed by means of the Structured Clinical Interview for DSM-MI-R (SCID)(Spitzer, Williams, \& Gibbon, 1987) (Dutch translation: Koster van Groos, 1985; Arntz, Bögels, \& Hoekstra, 1992-a). The mean duration of the main disorder was 6.6 years (range 1-26). The sample consisted of 18 males and 24 females. Their mean age was 33.4 years $(S D=8.4)$. Sixty-four per cent of the patients were married or living
together with a partner.

\section{Informants}

The intaker asked the patients to nominate a close informant according to the following criteria. The patient had to have had relatively intense contact with the 
informant on a regular basis for the last years, including periods of both bad and good moods of the patient. Above that, the patient had to have the impression that the informant knows how the patient behaved and felt in different situations (free from Axis I complaints). The informants who participated in the study ( $n=$ 42), consisted of 29 spouses, 8 parents, 1 other close relative and 4 close friends. The mean duration of acquaintance was 14.6 years $(S D=8.1)$.

\section{Assessment Instruments}

Interview for Personality Disorders. The (semi)Structured Clinical Interview for DSM-IIT-R Personality Disorders (SCID-II)(Spitzer, Williams, Gibbon, \& First, 1990) was used for the assessment of personality pathology (Dutch translation: PC Bloemendaal, 1991). The interviewer was instructed to discuss all SCID-II traits with the patient or the informant. Traits were rated as threshold or true (3), as subthreshold (2) or as absent (1). As in the DSM-III-R (American Psychiatric Association, 1987), the trait must be stable over time and situations, and not be merely present in the period of Axis I disorders. In the present study, the SCID-II interview with patients took more time than the interview with informants $M=$ 119 minutes $(S D=33)$ vs. $M=107$ minutes $(S D=21) ; t(41)=2.37, p=.02]$.

Questions for Measuring Intensity and Intimacy of the Relationship. Both patient and informant were asked to rate five questions concerning the intensity and intimacy of their relationship:

1 'How often does the intimate witness you in daily life, including good and bad moments?' (e.g. informant formulation: 'How often do you witness him/ her in daily life, including good and bad moments?').

2 "To what extent do you talk to the intimate about positive events that concern yourself?'.

3 'To what extent do you talk to the intimate about negative events that concern yourself (e.g. your failures or things you dread)?'.

4 "To what extent does the intimate know how you behave in different situations?'.

5 'To what extent does the intimate know how you feel in different situations?'.

Questions had to be rated on five-point scales ranging from 'never' to 'very often" or from 'not at all' to 'excellent'. Patient and informant mean ratings on all questions were larger than $3(3=$ "regularly' or 'reasonably well'), indicating that patients had nominated knowledgeable informants. There were no significant differences in the answers between patients and informants, except for Question 5, ind icating that informants overestimated their knowledge about feelings of the patient in different situations $[t(40)=-2.01, p=.05]$. Furthermore, there was a trend for differences in answer to Question $3[t(40)=-1.86, p=.07]$, indicating that informants overestimated the extent to which the patient has talked about personal negative events to them. After the SCID-II interview was held, Questions 4 and 5 concerning knowledge about behavior and feelings of the patients were repeated. The postinterview ratings did not differ from pre-interview ratings ( $p>>12$ ), indicating that neither patients nor informants changed these opinions after being interviewed.

Therapist Evaluations of Personality Pathology. By means of a self-constructed questionnaire therapists were asked to evaluate the personality of the patient, 
being blind to the SCID-II diagnoses. They had to indicate whether the patient had a PD according to DSM-M-R criteria (APA, 1987) or not. Furthemore, visual analogue scales (0-100 mm) had to be rated for every individual PD that is addressed in the sciD-I interview (e.g. "To what extent did the patient have an avoidant personality?" $\left({ }^{\prime} O^{\prime}=\right.$ not at all, ' $100^{\prime}=$ extremely). Therapists were not interviewed by means of the SCID-II interview, because the separate items require very detailed knowledge of the patient's feelings, thoughts and behavior. Therapists were not expected to have that kind of detailed knowledge, because in most cases therapy was focused on Axis I disorders.

\section{Raters of the SCID-II}

Raters of the SCID II were two mental health scientists (L.D. \& M.H.; degree is comparable to clinical psychology). They were familiar with DSM-IH-R Axis I and Axis II disorders (APA, 1987) and had experience in diagnosing and treating psychiatric patients. Before the start of the study they were trained extensively in administering the SCID-n by doing joint-interviews with experienced SCID-II interviewers, who had participated in a study showing very good interater reliabilities (Arntz et al., 1992-b). They were first and second rater by turn.s. Afterwards they discussed uncertainties and difficulties, and feedback was given to improve their elicitation and interpretation of information. Next, the two raters observed interviews of each other, and again, uncertainties and difficulties were discussed.

With due reserve, we estimated the short-interval test-retest interrater reliability between the two raters in a sample of eleven outpatients (the sample size was very small and no base rate requirements were made). Percentage observed agreement on item level ranged from 55\% to $100 \%$. Agreement on traitscores (number of traits present) was fair to excellent (ICCs $\geq 0.45$; Table 2 ) for most of the specific PD categories. In conclusion, agreement between the two raters in the present study seems to be adequate. Note that the ratings were based on independent interviews with the same patient, which is a more thorough examination of reliability than the joint-interview approach (Zimmeman, 1994).

\section{Procediure}

After referral to the 'Academic Section Behavior Therapy', patients were screened by an intaker, someone other than the two scid If raters, for the presence of Axis I disorders. Next, patients were asked to participate in the present study and to nominate an informant according to the criteria mentioned earlier. Writen informed consent was obtained from participating patients and informants.

The interviewers were informed about the presence and duration of Axis I disorders, about the age, profession, marital and family status of the patient, and about the length of their relationship with the informant.

For practical reasons patient and informant could not be interviewed simultaneously. We interviewed the informant before the patient, because we thought it was more likely that the informant would be influenced by the patient, than the other way around. The informant was asked not to discuss the interview with the pationt. The patient was interviewed by the second rater within two weeks of the first interview being administered. Each rater administered as many interviews with patients as with informants. The informant, patient and second rater 
were blind to the results obtained from the patient interview.

The questions for measuring quality of the relationship between patient and informant were answered just before starting the SCID-II interview. Two questions were repeated after the SCID-II interview.

The SCID-II interviews were taken before starting therapy. Approximately one year after starting therapy, the therapist was asked to complete the questionnaire about the personality of the patient. The therapist was blind to the SCID-II diagnoses of the patient.

\section{Statistical Analyses}

Intraclass Correlation Coefficient (ICC; Fleiss, 1986) was used for estimating agreement on the single SCID-II items (true, subthreshold, or absent) and the traitscores (number of positive traits of specific PDs). Cohen's kappa (Cohen, 1960) was used for estimating agreement on the presence of a PD. Generally, ICC and Kappa values larger than 0.75 are taken to indicate excellent agreement, values below 0.40 indicate poor agreement, and values in between indicate fair to good agreement (Fleiss, 1981; Landis \& Koch, 1977). Kappa and ICC are influenced by the illness base rate. When the base rate is low, a few disagreements can have a marked impact on the overall level of agreement (Zimmerman, 1994, p. 226). Therefore, kappa and ICC were calculated only for those traits and PDs with at frequency of at least five positive cases, either by the patient or the informant interview (Zimmerman, 1994, p. 226). ICC for specific traitscores was calculated if at least five cases were diagnosed with two or more of those traits, either by the patient or the informant interview.

\section{Results}

\section{Patient-Informant Concordance}

Single Traits. ICC was calculated for 59 out of the 108 different SCID-Il traits (Table 1). The majority of traits reached poor agreement $(\mathrm{ICC}<0.40)$. Eleven traits reached fair to good agreement $(0.40<$ ICC $<0.75)$ and only one trait reached excellent agreement (borderline $6,1 C C=0.78$ ). The borderline traits scored highest on the number of items with adequate agreement ( 3 items out of 8 ).

Traitscores of PD Categories. Agreement for paranoid and borderline traitscores was fair to good ( $\mathrm{ICC}=0.61,1 \mathrm{CC}=0.47$, resp.; Table 2$)$. All other calculated traitscores of specific PD categories reached only poor agreement (iccs $<0.40$; Table 2 ).

$D S M-H I-R$ PDS. Diagnostic agreement for the presence of an avoidant PD was very poor $(K=0.05)$. The same held for the presence of any $P D$, regardless of type $(K=0.06)$. For the other PDs kappa was not calculated. Observed agreement on the presence of specific PDs ranged from $74 \%$ to $100 \%$. 


\section{TABLE 1}

Concordance between patients and informants $(n=42)$ on the SCID 11 traits

\section{Porsonality disonder}

Avoidant 17 traite)

Dependent 19 traits:

Obsessiwe compulsiwe 19 traits

Passive aggressive 19 traits

Selfodefeating (8 thraits)

Paranoid (7 traits)

Schizotypal 19 traits!

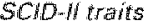

1. Easily hurt by criticism

2. No intimate friends

3. Avoids personal contact with people

4. Avoids socialutalk activities

5. Reticent in social situations

6. Fears of being embarrassed

7. Avoid any ristks

1. Unable to make everyday decisions

2. Permits others to make major decisions

3. Always agrees

4. Difficulty initiating projects or doing thirgs on

his or her own

5. Does unpleasant jobs in order to be liked

7. Feels helpless when close relationships end

8. Preoccupied with fears of being abandoned

9. [see avoüdant personality disorder trait 1)

1. Parfectionist

2. Preoccupied with details

3. Insists that others do things this or her way

4. Excessivelly devoted to work

5. Inderisive

6. Owerconscientious about matters of morality, etc.

7. Restricted expression of affection

9. Saves worthless things

1. Procrastinates

2. Irritable when something unpleasant is asked

5. "Forgets" obligations

6. Feels underappreciated

7. liritated by suggestions

9. Scorns authorities unreasonably

1. Chooses disappointments, faifures etc.

2. Riejects help

3. Depressive when successful

6. Fails to accomplish crucial tasks

8. Excessive self-sacrifice that is unsolicited

11. Expects to be exploited

2. Questions the lovalty of friendls or colleaguen

3. Sees hidden dangers

4. Unforgiving

5. Trusts no one

6. Easily silighted and angry

7. Questions fidellty of partner without justification

1. Ldeas of reference

2. Excessive sociall anxiety

3. Magica think king

4. Unusual perceptuall experiences

6. isee avoidant personality disorder trait 2

9. (at least one of the first five paranoid tiraits)
$1 \mathrm{CC}^{2}$

0.08

0.31

0.30

0.26

0.25

0.18

0.11

0.01

0.21

$-0.03$

0.21

0.15

0.16

$-0.03$

0.31

0.71

0.34

0.34

0.15

0.10

0.20

0.34

0.26

0.08

$-0.22$

0.39

$-0.20$

0.59

0.41

0.14

$-0.03$

0.26

0.17

0.26

0.64

$-0.13$

0.37

0.35

0.57

$-0.03$

0.00

0.06

0.19

0.51 
Table 1 (continued)

Personality disorder

Schizoid 17 traits

Histrionic (8 traits)

Narcissistic (9 traits)

Borderline (9 qraits)

Antisocial B (12 traits)

Antisocial C (10 traits)

Sadistic (8 traits)
2. Chooses solitary activities

4. No desire for sexual relations

0.07

6. (see avoidant personality disonder trait 2 )

-

3. Overly concemed with appearance

7. Selfwcentered

0.13

1. Angry after criticism 0.00

7. Requires constant admiration $\quad-0.08$

1. Unstable relationships 0.44

2. Self-damaging impulsivity $\quad 0.67$

3. Affective instability $\quad 0.22$

4. Lack of anger-control 0.28

6. Udentity disturbances $\quad 0.78$

7. Chronic feetings of emptiness 0.32

1. Often truant 0.35

11. Repeated stealing 0.55

7. Reckless 0.57

$\mathrm{ICC}=$ Intraclass Correlation Coefficient (random raters)

Antisocial $\mathrm{B}=$ antisocial behavior betore age 15

Antisocial $C=$ antisocial behavior since the age of 15

a $I C C$ for single traits was calculated if at least 5 cases were rated positively $(=3$ enther in the group of patient-interviews or in the group of informant interwiews. Furthermore, ICC cannot be calculated if the variance in one of the groups is equal to zero

TABLE 2

Concordance between the two raters $(n=11)$ and between patients and informants $(n=42)$ on SCID-II traitscores

Conicordance
between rateirs
1CC
trattscore b
0.95
0.85
0.64
0.08
0.37
0.52
0.66
0.45
0.88
0.67
0.45
-0.08
0.12

Concordance between

\section{Personality disorder}

Avoidant

Dependent

Obsessive compulsive

Passive aggressive

Self-defeating

Paranoind

Schizotypal

Schizoid

Histrionic

Narcissistic

Borderline

Antisociall B

Antisocial C

\begin{tabular}{|c|c|}
\hline $\begin{array}{l}{ }^{1 C C} C^{a} \\
\text { tratscone b }\end{array}$ & $\begin{array}{l}\text { "Ovality of velationsthip } \\
\text { low/high" }\end{array}$ \\
\hline 0.27 & $(0.21 / 0.35$ \\
\hline 0.25 & $(0.05 / 0.47)$ \\
\hline 0.39 & $(0.25 / 0.64)$ \\
\hline 0.12 & $(-0.03 / 0.26)$ \\
\hline 0.34 & $(0.50 / 0.23)$ \\
\hline 0.61 & $(0.48 / 0.80)$ \\
\hline 0.28 & $(0.18 / 0.33)$ \\
\hline- & - \\
\hline- & - \\
\hline - & - \\
\hline 0.47 & $(0.16 / 0.65)$ \\
\hline - & 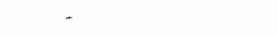 \\
\hline- & - \\
\hline
\end{tabular}

$\triangle C C=$ Intraclass Correlation Coefficient

Antisociall $B=$ antisocial behavior before age 15

Artisocial $C=$ antisocial behavior since the age of 15

a ICC (random raters) was calculated if at least five cases were diagnosed with two or more of those traits either by the patient oir the informant int revilew

Thaitscore is the number of positive traits of a specific person ality disorder 


\section{Prevalence Rates in Patient and Informant Interviews}

Patient interviews tended to yield higher rates of personality diagnoses than informants (Table 3). However, McNemar's test for frequency (McNemar, 1969) indicated no significant differences between groups with respect to the presence of any PD or with respect to specific PDs ( $p s>.05$ ). There was a trend for avoidant $\mathrm{PD}$ to be diagnosed more frequently in the patient interviews than in the informant interviews $(p=.07)$. Regarding traitscores, patient interviews yielded higher ratings than informant interviews for the avoidant, self-defeating and schizotypal traitscore ( $p s<.006$; Table 3). In all other cases, patient interviews did not differ significantly from informant interviews ( $p s>05$; Table 3 ).

\section{TABLE 3}

Frecuency of DSM-IILR personality disorders and mean traitscore for specific personality disorders in patient and informant interviews

\begin{tabular}{|c|c|c|c|c|c|c|c|c|}
\hline \multirow[b]{2}{*}{ Personatity disorder } & \multicolumn{4}{|c|}{$\begin{array}{l}\text { Frequiency of DSM-III-R } \\
\text { Personality disorders }(\%)\end{array}$} & \multicolumn{4}{|c|}{ Meain traisscore a (SD) } \\
\hline & \multicolumn{2}{|c|}{$\begin{array}{l}\text { Patient } \\
\text { interview }\end{array}$} & \multicolumn{2}{|c|}{$\begin{array}{l}\text { Informant } \\
\text { interview }\end{array}$} & \multicolumn{2}{|c|}{$\begin{array}{l}\text { Patient } \\
\text { interview }\end{array}$} & \multicolumn{2}{|c|}{$\begin{array}{l}\text { Informant } \\
\text { interview }\end{array}$} \\
\hline Avoidant & 10 & (24) & 3 & $(7))^{b}$ & 2.1 & $(1.8)$ & 1.2 & $(1.3)^{\mathrm{d}}$ \\
\hline Dependent & 4 & $(10)$ & 2 & (5) & 1.7 & $(1.6)$ & 1.2 & $(1.3)$ \\
\hline Obsessive compulsive & 3 & (7) & 1 & $(2)$ & 1.2 & $(1.6)$ & 1.2 & $(11.4)$ \\
\hline Passive aggressilwe & 1 & $|2|$ & 1 & (2) & 0.7 & $(1.2)$ & 0.7 & $(9.1)$ \\
\hline Self-defeating & 0 & $(0)$ & 0 & 0 & 0.6 & $11.0)$ & 0.2 & $(0.4)^{\mathrm{d}}$ \\
\hline Paranoid & 0 & (0) & 3 & (7) & 0.9 & $(1.2)$ & 0.7 & $(1.3)$ \\
\hline Schizolypal & 1 & (2) & 0 & $(0)$ & 1.1 & $(1.2)$ & 0.5 & $(0.8)^{d}$ \\
\hline Schizoid & 0 & 0 & 1 & $(2)$ & 0.3 & 10.4 & 0.3 & 10.71 \\
\hline Histrionic & 0 & (0) & 0 & $10 !$ & 0.3 & $(0.6)$ & 0.3 & $(0,6)$ \\
\hline Narcilssistic & 0 & 101 & 0 & 101 & 0.3 & $(0.6)$ & 0.4 & $\langle 0.7\rangle$ \\
\hline Borderline & 3 & (7) & 1 & 2 & 0.8 & $(1.6)$ & 0.5 & $(1.1)$ \\
\hline Antisocial B & 0 & (10) & 0 & 101 & 0.4 & $(0.9)$ & 0.3 & $(0.7)$ \\
\hline Antisocial C & 0 & (10) & 0 & 10 & 0.2 & $(0.5)$ & 0.2 & $(0.7)$ \\
\hline Any $\mathrm{PD}$ & 16 & $|38\rangle$ & & $(21)^{\mathrm{C}}$ & & & & \\
\hline
\end{tabular}

Antisocial $B=$ antisocial behavior before agie 15

Antisocial $C$ antisocial behavior since the age of 15

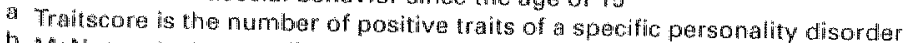

b MoNamar test, two-tailed, to compare trequencies. $p=.07$

c MoNomar test, wortalled, $\mathrm{p}=.94$

d perired T-test, two-tailed, $p \leq .01$

Differences in prevalence raise the question of whether data derived from interviews with informants are merely a subset of what is derived from patient interviews, or whether informant interviews yield new information. In total, informant interviews resulted in 12 PD diagnoses, of which only 2 diagnoses $(16.7 \%)$ were also made on the basis of direct interviews with patients. With regard to all positively rated traits derived from informant interviews, there was only $36 \%$ overlap with the data based on direct interviews with patients. Avoidant and paranoid traits had the largest percentage overlap (above 50\%). Regarding passive aggressive, self-defeating, schizoid, histrionic and narcissistic traits, less than $25 \%$ 
of the traits rated positively were also derived from direct patient interviews. In short, informant based diagnoses are not merely a subset of patient based diagnoses, but they contain new information.

\section{Factors Related to Concordance between Patient and Informant Interiews}

Three factors were considered to be related to concordance between patient and informant interviews: behavioral versus internal-attitudinal formulation of the traits, degree of social undesirability, and finally, the quality of the relationship, expressed in the degree of intensity and intimacy.

Two of us (L.D. \& A.A.) categorized 17 out of the 59 traits as behaviorally and 22 as internal-attitudinal. The remaining 20 traits were considered in between these two categories (e.g. behaviorally formulated, with a specific hidden motive that had to be checked by the interviewer). The mean Iccs did not differ across the categories [resp. $1 \mathrm{ICC}=0.20(S D=0.20), \mathrm{ICC}=0.28(S D=0.16), \mathrm{ICC}=0.22(S D=0.22)$; $F(2,56)=0.69, p=.51]$.

Next, two of us (L.D. \& A.A.) categorized 48 out of the 59 traits as slightly socially undesirable and 11 as highly socially undesirable. Again, mean ICCs did not differ across the categories [resp. $I C C=0.22(S D=0.20), \mathrm{ICC}=0.28(S D=0.34)$; $t(11.55)=-0.53, p=.60]$.

Finally, the quality of the relationship was considered as a factor related to concordance. Patient and informant scores on the 5 questions concerning the intensity and intimacy of the relationship were added, and using a median split, we divided couples into two groups. Couples with highly intense and intinate relationships generally reached higher agreement on the traitscores (4. traitscores: ICCS > 0.40; Table 2) than couples with relatively less intense and intimate relationships ( 2 traitscores: ICCs $>0.40$; Table 2 ).

Therapist Evaluations of Personality Pathology Compared to Patient-and Informant Based SCID-II Diagnoses

According to the evaluations of the therapists, $15(36 \%)$ patients had a DSM-III-RR PD $(A P A, 1987)$. Diagnostic agreement on the presence of any DSM-III-R PD between patient interviews and therapist ratings was moderate $(K=0.44)$. Diagnostic agreement between informant interviews and therapist evaluations was very poor $(K=-0.02)$. Pearson correlations between patient based traitscores and therapist evaluations were significant for the self-defeating, paranoid, schizotypal, schizoid and borderline variables ( $p s<05$; Table 4 ). Pearson correlations between informant based interviews and therapist evaluations were mostly non-significant ( $p s$ $>.05$ ), except for the borderline variables (Table 4).

In short, patient-therapist correlations were somewhat higher than informanttherapist correlations, indicating that therapist ratings have relatively more in common with diagnoses derived from patient interviews than with diagnoses from informant interviews.

Table 4 also shows correlations between patient and informant based SCID-II correlations. They are generally higher than the correlations with the therapist evaluations, possibly because they are based on the same assessment instrument. 
TABLE 4

Correllations between therapist evaluations, patient based SCID-II traitscores and informant based SClD-1l traitscores

\begin{tabular}{|c|c|c|}
\hline & Pearson comalations & \\
\hline Patrent-therapist & Informant therapist & Patient-informant \\
\hline 0.23 & 0.18 & $0.31 *$ \\
\hline 0.00 & 0.08 & 0.23 \\
\hline 0.22 & -0.03 & $0.39^{m-k}$ \\
\hline 0.01 & 0.07 & 0.11 \\
\hline 0.314 & 0.06 & $0.56^{* * *}$ \\
\hline 0.34 & 0.24 & $0.62 * *$ \\
\hline $0.56 * *$ & 0.14 & $0.32 *$ \\
\hline $0.36^{*}$ & 0.11 & $0.33^{*}$ \\
\hline-0.08 & -0.01 & 0.16 \\
\hline-0.02 & 0.17 & 0.22 \\
\hline $0.72^{m * * * *}$ & $0.27 *$ & $0.50^{* * * *}$ \\
\hline-0.12 & .0 .08 & $0.60 * *$ \\
\hline
\end{tabular}

Antisociall $C=$ antisocial behtavior since the age of 15

a Traitscone is the number of positive traits of a specific personality disorder

* pe.05, one-trailed

* $\mathrm{p} \leq \mathrm{0} .01$, one-talled

F* $\mathrm{p}<001$, one thailed

\section{Discussion}

The (semi)Structuned Clinical Interview for DSM-II-R Personality Disorders (SCIDii)(Spitzer et al, 1990) was used to examime the concordance between patient and informant interviews in the assessment of DSM-II-R personality pathology (APA. 1987) in outpatients. Similar to prior studies, low or only modest levels of agreement were found for the single traits, the traitscores, and the PD diagnoses that were examined. High levels of agreement (convergent validity) would have been a welcome indication of the validity of patient reports in the assessment of personality pathology. Low levels of agreement, on the other hand, do not prove that patient reports are invalid in the assessment of personality pathology. Low levels of agreement raise the question which source of information is more valid.

To our knowledge, this is the first study that attempted to determine which source of information is more valid, patients or informants. Diagnoses from the patient and informant interview were compared to therapist evaluations of the personality of the patient. Patient-therapist Pearson correlations were somewhat ligher than informant-therapist correlations. Patient-therapist agreement on the presence of any $\mathrm{PD}$ was fair to good, whereas informant-therapist agreement was very poor. These findings seem to indicate that patients are a more valid source than intormants (intimates). However, it does not exclude the possibility that both patients and therapist are invalid sources of information.. Therapists had to rely on the patient as the source of information, which might have induced agreement. Another reason to distrust therapist ratings is that clinically based diagnoses generally have poor reliability (Mellsop, Varghese, Joshua, \& Hicks, 1982; Pilkonis, Heape, Ruddy, \& Serrao, 1991; Shea et al., 1990; Spitzer, Forman, \& Nee, 1979). 
Before drawing the conclusion that patients are a more valid source of information than informants, more evidence is needed. More evidence can be obtained by studying potential external validators, such as treatment responsivity.

Quality of the relationship was found to be related to concordance. Couples with highly intense and intimate relationships generally reached higher and more satisfying agreement than couples with relatively less intense and intimate relationships. This increase in convergent validity is a promising finding in the assessment of personality pathology. It can be considered as an indication for the validity of SCID-II interviews relying only on patient reports. That is, at least for patients who are able to maintain intense and intimate relationships.

In the present study, patient interviews tended to yield more symptoms for some of the PD categories than informant interviews. Since previous studies (Dowson, 1992; Riso et al., 1994; Steketee, 1990; Tyrer et al., 1979; Zimmerman et al., 1988) have reported conflicting results as to which source of information yielded more personality pathology, prevalence differences between patient and informant reports have to be interpreted with caution. Explanations that might have caused patients to report more personality symptoms than informants are the following. (1) Informants were generally better able to distinguish the normal personality from the episodic Axis I disorder, whereas patients were biased by their Axis I complaints. (2) Informants lacked detailed information about the present and past life of the patient. Disagreement on two questions conceming the quality of the relationship also suggest that informants lacked some knowledge about feelings and negative events concerning the patient. The finding that concordance increased when the intensity and intimacy of the relationships was high, is also in favor of this explanation. (3) Informants only reported pathology that was severe and dysfunctional, and therefore perceptible to intimates. The finding that there was no difference in concordance rates between behavioral criteria and internal-attitudinal criteria, as was also found by Zimmerman et al. (1988) and Riso et al. (1994), does not necessarily contradict this explanation. Formulation of criteria in terms of overt behavior does not automatically mean that the behavior will be noticed by intimates. In fact, one could argue that all DSM-III-R criteria will become perceptible to close others as soon as they are very severe or dysfunctional.

Besides prevalence differences, it was also found that informant interviews yielded a substantial amount of information that did not emerge from patient interviews. Explanations of what might have caused informants to reveal new information include (1) patients underreported symptoms due to shame or egosyntonicity, and (2) the personality pathology of informants themselves biased their report, resulting in exaggeration or overstatement. Personality pathology of informants was not assessed in the present study.

In contradistinction with the 'shame hypothesis, we found that the mean ICC of highly socially undesirable traits did not differ from the mean ICC of the remaining traits. However, all SCID II traits are formulated in extreme terms and therefore it is quite conceivable that they are unwanted, especially by the person concerned. It would be interesting to study whether patient-informant Iccs increase with personallity traits that are not socially undesirable.

Several limitations of the study should be noted. First, the study focused only on outpatients, mostly with Axis I anxiety disorders. The findings cannot be 
generalized to inpatients who are more severely ill, or to outpatient samples with other DSM Axis I-diagnoses (APA, 1987, 1994), such as depression. Second, we tried to minimize the problem of low variance, by excluding traits and disorders with a very low positive base rate from the analyses. However, due to the nature of the sample (psychotherapy outpatients with mostly anxiety disorders) as well as to the relatively small sample size, several traits were still diagnosed relatively infrequently, which means a few disagreements can have a marked impact on the level of agreement. Third, with a relatively small sample size, concordance coefficients have a large standard error of measurement and thus are less stable (Fleiss, 1981). Results based on a relatively small sample size should therefore be interpreted more cautiously than results from larger samples, and replication is needed, combined with attempts to determine which source of information is more valid (patients or informants).

Taken together, the present study does not show overwhelming evidence for or against the validity of SCID-II diagnoses based only on patient reports. Agreement between patient and informant interviews was low. Comparisons with therapist evaluations suggested that patient interviews are more valid than informant interviews. The increase in convergent validity as a result of the increase in quality of the relationship is also promising. Nevertheless, the lack of a golden standard forces us to find more evidence for the validity of patient reports on personality pathology. Additional evidence may result in studying predictive validity of patient based diagnoses and informant based diagnoses.

As a final comment, we want to stress that by definition DSM criteria for PDS refer to traits that cause significant impairment in social or occupational functioning or subjective distress. Traits should be exhibited in a widle range of important social and personal contexts (APA, 1987, 1994). We reason that informants who interact with the patient frequently must notice personality psychopathology that interferes with many areas of daily functioning by definition. If informants do not report personality pathology, one might infer that the personality characteristics of the patient are not severe enough to be considered as a disorder. With patient interviews, one might introduce the requirement that positive ratings are made only when traits are perceptible to others. It would be very interesting to re-evaluate concordance between patient and informant interviews, using this requirement
in the patient interviews. 


\section{note}

1. Published as: Dreessen, L., Hildebrand, M. \& Arntz, A. (1998 a). Patient-informant concondance on the Structured Clinical Interview for DSM-III-R Personality Disorders (SCID-II) fouman of Personality Disorders, 12, 149-161. 


\section{The impact of personality disorders on cognitive and behavior therapies for anxiety disorders}

In Chapter 3, four studies are presented in which the impact of personality disorders on cognitive and behavior therapies for various anxiety disorders was investigated. Three studies focus on therapy outcome and one on therapy process. Section 3.1 deals with therapy outcome in panic disorder and in various anxiety disorders lumped together as a group. Section 3.2 deals with therapy outcome in obsessive compulsive disorder. The fourth study in section 3.3 addresses the impact of personality disorders on the therapy process in the treatment of various Axis I anxiety disorders lumped together as a group. 


\section{Personality disorders do not influence the results of cognitive and behavior therapies for panic disorder and for various anxiety disorders lumped together as a} group

\section{Summary}

Two studies are presented in which the presence of personality disorders (PDs) was assessed using a structured dinical interview (Structured Clinical Interview for DSM-III-R Personality Disorders (SCID-II]) by an independent rater before the start of treatment. The therapy effect was measured by change scores. In the first study, patients $(n=31$ ) with panic disorder (with/without agoraphobia) received standardized cognitive behavioral treatinent protocols: The effect of treatment as assessed by questionnaires and panic frequency was comparable in the groups with and without $2 D$. In a second study, patients $(n=57)$ with various anxiety disorders were assessed before and after an individually tailored cognitive behavioral treatment. In general, results showed that anxiety patients with PDs have more severe Axis I pathology, but show a change parallel to the patients without PD. Thus, if the effect of therapy is measured by change scores, PD is not related to therapeutic failure of cognitive behavioral treatment of anxiety disorders in general and panic disorder in particular:

\section{Introduction ${ }^{2}$}

In clinical practice, it is often assumed that patients with anxiety disorders on Axis I and a concomitant personality disorder (PD) have a poor treatment prognosis. Various difficulties have been described: low tolerance for strong emotions; poor compliance with homework assignments; questionable motivation for change; relational difficulties with the therapist; and complaints related to complex, deeply ingrained, inflexible, maladaptive patterns (Padesky, 1988).

Previous empirical studies seem to substantiate this view largely, but not unanimously. Seventeen studies were traced addressing the issue of the influence of PD on the effects of psychotherapeutic and/or pharmacological treatment of anxiety disorders. Twelve studies report a negative influence of $\mathrm{PD}$ on the effects of therapy for different anxiety disorders (Chambless, Renneberg, Goldstein, \& Gracely, 1992; Farvelli \& Albanesi, 1987; Green \& Curtis, 1988; Hermesh, Shahar, \& Munitz, 1987; Kringlen, 1965; Mavissakalian \& Hamann, 1987; Minichiello, Baer, 
\& Jenike, 1987; Numberg et al, 1989; Reich, 1988; Reich, 1990; Tumer, 1987; Tyrer, Casey, \&all, 1983). Three studies report a positive effect (Lo, 1967; Rabavilas, Boulougouris, \& Perrisaki, 1979; Vaughan \& Beech, 1985), and two report no effect at all (Mavissakalian, Hamann \& Jones, 1990; Steketee, 1990). In general, several methodological problems of these studies can be noted. First, a retrospective diagnosis of PDs by the therapist (Minichiello et al, 1987; Numberg et al, 1989), the investigator (Turner, 1987), or the rater (Vaughan \& Beech, 1985) not blinded to the effects of treatment might yield confounded results; more Axis II disorders might be diagnosed in therapy failures because of the therapeutic failure. It might be argued that the therapist can make a more valid Axis I diagnosis during therapy because of more knowledge of the patient's behavior, thoughts and feelings. However, such a diagnosis would undoubtedly be influenced by the patient's progress in therapy. A sensible use of the concept of PDs implies that these disorders are defined by criteria other than lack of therapeutic progress.

Second, questionnaires, as were used in several studies (Chambless et al., 1992; Faravelli \& Albanesi, 1987; Mavissakalian \& Hamann, 1987; Mavissakalian et al., 1990; Reich, 1990; Steketee, 1990), tend to overdiagnose (Chambless et al., 1992; Repko \& Cooper, 1985) and are more liable to confounding influences of $A x$ is $\mathbb{I}$ disorders than clinical interviews (Joffe \& Regan, 1988; Noyes, Reich, Suelzer, \& Christiansen, 1991). Although clinical interviewers are believed to have a tendency to underdiagnose (Hyler et al, 1987; Widiger \& Frances, 1987), it is generally assumed that interviews yield more valid Axis II diagnoses than questionnaires.

Third, only five studies report on whether therapist or patient were given any information about the Axis II diagnosis (Chambless et al, 1992; Reich, 1988; Reich, 1990; Vaughan \& Beech, 1985). Ideally, studies are double-blind.

Fourth, several studies did not use validated measurement instruments for Axis I complaints (Kringlen, 1965; Lo, 1967; Minichiello et al., 1987; Vaughan \& Beech, 1985).

Fifth, the way in which the treatment effect is quantified might obscure improvement in some studies. When effects are measured in a dichotomous way (Green \& Curtis, 1988; Kringlen, 1965; Lo, 1967; Mavissakalian \& Hamann, 1987; Mavissakalian et al., 1990; Minichiello et al., 1987; Tyrer et al., 1983; Vaughan \& Beech, 1985), the patient is either cured or not. A dimensional approach might be mone suitable for measuring the severity of anxiety and fear; people are not either anxious (and therefore iil) or not anxious at all (and therefore healthy). Improvement is also quantified by the number of and/or severity of symptoms after therapy (end-state functioning with or without pretest as covariate) (Faravelli \& Albanesi, 1987; Numberg et al., 1989; Reich, 1988; Reich, 1990). A pretest-posttest design is preferable because it can be expected that patients with $P D$ have more Axis I complaints at pretest. In that case, patients with PD can have higher symptom scones after therapy, but compared with pretest scores, they might have improved parallel to patients without PD. Improvement expressed as a percenLage, $[$ (posttest-pretest)/pretest $\mid \times 100 \%$ (Green \& Curtis, 1988; Reich, 1988; Tyrer et $1 ., 1983$ ), is also problematic if the groups (PD and no PD) differ at pretest. With a high pretest score, a decrease of, say, $50 \%$ is a much larger decrease than a decrease of $50 \%$ with a low pretest score. This would mean that if PD patients have substantially higher pretest complaint scores than patients without PD, their 
improvement could be less when expressed as percentages but larger when expressed as raw change scores. In sum, several studies measured improvement in such a way that possible pretest differences are not taken into account or might obscure improvement. In our opinion, improvement should be measured as the change from pretest to posttest (Chambless et al., 1992; Steketee, 1990).

Taken together, given the various methodological problems, it seems difficult to draw firm conclusions from previous research on the influence of PDs on treatment results. Hitherto, only five studies used a structured interview (Green \& Curtis, 1988; Nurnberg et al., 1989; Rabavilas et al., 1979; Reich, 1988; Tyrer et al., 1983), of which only one investigated behavior therapy (Rabavilas et al., 1979). However, this study has a very small sample size $(n=12)$ and no between group test differences are reported. Therefore, further studies into the influences of PDs on the effects of cognitive behavior therapies for anxiety disorders are needed.

Two studies are presented regarding anxiety patients who had been referred for cognitive behavioral treatment in the Community Mental Health Center Maastricht. In the first study (study 1), the effects of PDs on short-term standardized cognitive behavioral treatment protocols for panic disorder are explored. In the second study (study 2) the effects of PDs on the results of individually tailored cognitive behavioral treatments for various anxiety patients are investigated. Improvement is investigated as relative improvement by means of change scores. We reasoned that not the absolute level of functioning at the end of therapy should be the indicator of success, nor the percentage of improvement, but the amount of improvement.

\section{Study one}

The influence of PDs on short-term cognitive behavioral treatment protocols for panic disorder

\section{Method}

\section{Participants}

Participants were 31 patients who had been referred for cognitive behavioral treatment at the 'Academic Section Behavior Therapy' of the Community Mental Health Center for outpatients at Maastricht. The patients met the DSM-III-R criteria (American Psychiatric Association, 1987) for panic disorder with $(n=16)$ or without agoraphobia $(n=15)$ based on an unstructured interview. Of all participants in the present study, 17 were diagnosed as having no PD and 14 as having one $(n=$ 11 ) or more $(n=3)$ PDs (based upon the Structured Clinical Interview for DSM-111$\mathrm{R}$ Personality Disorders [SCID-II], see below). Of the patients with one PD, two were diagnosed as paranoid, six as avoidant, and three as dependent. Among the patients with more than one PD, there were two paranoid, two borderline, three dependent, two obsessive compulsive, one passive aggressive, and one self-defeating $\mathrm{PD}(\mathrm{s})$.

In the non-PD group, $53 \%$ were male, $64.7 \%$ were married or living with a partner, and $70.6 \%$ were employed. Their mean age was $33.4 \pm 7.4$ years, and the mean duration of the Axis I illness was $5.3 \pm 5.2$ years. In the PD group, $28.6 \%$ were male, $71.4 \%$ were married or living with a partner, and $78.6 \%$ were employed. Their mean age was $37.4 \pm 9.6$ years, and the mean duration of the Axis I illness was 6.5 
\pm 5.5 years. The $\mathrm{PD}$ group did not differ significantly from the non-PD patients on any of the demographic variables (all $p s>.15$ ).

\section{Assessment Instruments}

A Dutch version of the SCrD-II (Spitzer, Williams, Gibbon, \& First, 1990) was used to diagnose the PDs. Briefly, the interview consists of a large number of questions corresponding to the DSM-III-R criteria for PDS (APA, 1987). The score of absence or presence of each criterion is based on discussion with and observation of the patient. When the specified number of criteria is present, the diagnosis of the corresponding $\mathrm{PD}$ is made. The SCID-II can assess $13 \mathrm{PDS}$, of which the interrater reliability in our group of raters has been found to have a kappa of 0.80 , on average (Arntz et al., 1992-b). The factorial validity has been tested in a large sample of patients by confirmatory factor analysis (Eussen, Arntz, \& Hoekstra ${ }_{y}$ 1991). Results were moderate to good. Thus, the SCID-II gives a reasonable indication of the presence and absence of PDs.

The treatment outcome measures included various self-report questionnaires: the Fear Questionnaire (FQ; Marks \& Matthews, 1979), of which avoidance of situations related to the main complaint and of agoraphobic situations were analyzed; the Fear of Fear (FOF; Van den Hout, Van der Molen, Griez, \& Lousberg, 1.987) inventory, which measures fear of bodily sensations that accompany panic and anxiety; the State-Trait Anxiety Inventory (STAI: Spielberger, Gorsuch, \& Lushene, 1970; Van der Ploeg, Defares, \& Spielberger, 1980), which measures present anxiety and the personality disposition to react with anxiety; and finally, the Beck Depression Inventory (BDr; Beck, Rush, Shaw, \& Emery, 1979). The mean number of full-blown panic attacks was also measured by a diary that the patient had to fill in everyday.

\section{Procedure}

The treatment study was controlled. Patients with panic disorder with agoraphobia received either 15 sessions of an exposure in vivo treatment or 15 sessions of cognitive therapy (Clark \& Salkovskis, 1991) combined with an exposure in vivo treatment. Patients with panic disorder without agoraphobia received either 12 sessions of applied relaxation (Öst, 1987) or 12 sessions of cognitive therapy (Clark \& Salkovskis, 1991). Patients did not receive any medication, or if they were already taking medication, it was tapered off before the start of treatment or kept at the same dosage during the treatment period. During the month following the treatment period, patients did not receive any additional treatment. After this month, additional treatment was administered if clinically indicated. Before the start of treatment, an independent rater administered the SCID-II to diagnose the $\mathrm{PD}(\mathrm{s})$, without informing the therapist or the patient of the outcome.

Before the start of the treatment, at the end of the standardized treatment, at 1 month follow-up evaluation (FU-1), and at 6-month follow-up evaluation (FU-2), the patients completed the various questionnaires mentioned above. The panic diary had to be completed, starting from 1 week before the treatment and continuing throughout the whole standardized treatment period. Panic frequency during the week before the treatment and during the last week of the standardized treatment was analyzed. Because of missing data, the number of patients for which panic frequency is analyzed is smaller than the total sample size. 


\section{Results}

Data were analyzed with multivariate analyses of variance. Treatment outcome was investigated as the change relative to the pretest scores. Accordingly, it is not the absolute level of mental health that was laken as an indicator of success, but the amount of improvement. The factor of interest to be tested was the effect of any $\mathrm{PD}$. Although initially the study was not designed to test the interactions between the presence of PDs and the type of treatment (cognitive vs behavior therapy). we determined whether any of the interactions at posttest or Fu- 1 were significant. With regard to the analyses of the effect of any $\mathrm{PD}$, the interaction factor was included in the multivariate analyses of variance only in case of a significant interaction effect.

\section{Interaction Effects}

At posttest and FU-1, the interactions between the presence of PDS and the type of treatment (cognitive us behavior therapy) were not significant ( $p s>10$ ), with the exception of state anxiety $(F=4.21, p=.05)$ and depression $(F=4.99, p=.03)$ at posttest. On these measures, PD patients benefited significantly more from cognitive than from behavior herapy (at pretest there were no significant differences). However, the univariate $F$ statistics did not reach the Bonferroni-corrected level of significance (corrected alpha $=.007$ ). Multivariate F statistics were not significant.

After Fu-1, additional cognitive belhavioral treatment was administered if clinically indicated, but these treatments were not standardized anymore. Therefore, it is not possible to analyze interaction effects at $\mathbb{F} U-2$.

\section{Effects of any PD}

Table 1 presents the multivariate (Wilks) and univariate F statistics for all questionnaires, and the univariate F statistic for the panic frequency. In light of the modest sample size and the explorative character of the study, alpha was set at .05 for all (two-tailed) tests. Figure 1 shows the mean ratings before and after the standardized treatment and at the follow-up tests. At pretest, patients with $\mathrm{PD}$ (s) had significantly higher trait anxiety ratings (STAI) than patients without a $\mathrm{PD}$, and were marginally significantly more depressed (BDI) than patients without a PD (Table 1). Howevel, the univariate $\mathbb{F}^{*}$ statistics failed to reach a Bonferronicorrected level of significance (corrected alpha $=.007$; the overall protection level is then .05). Scores on the other questionnaires and the panic frequency were not significantly different among the two groups. The multivariate F statistic was not significant, which indicates that the higher trait anxiety ratings of patients with PD(s) might be a chance finding.

At posttest, patients with $\mathrm{PD}(\mathrm{s})$ had improved in parallel to the patients without a PD: neither the multivariate nor the univariate $F$ statistics were significant. The same holds for the two follow-up periods. The two groups were still in parallel (Table 1). There was no evidence for the patients with $\mathrm{PD}(\mathrm{s})$ having a less stable improvement long-term. After Fu-1, additional treatment was provided if this seemed clinically indicated. Patients with and without $P D(s)$ did not differ in the total number of sessions $[t(1,29)=.62, p=.54]$ and the total duration of treatment [in months; $t(1,29)=.20, p=.84]$. 
In short, patients with panic disorder with or without agoraphobia and a concomitant PD profit equally from cognitive behavioral treatment focused on the disorder on Axis I. Treatment outcome is stable long term (at least 6 months).

\section{TABLE 1}

Study 1: Eftects of PD on pretest and treatment outcome of cognitive behavioral treatiment of panic disoirder

\begin{tabular}{|c|c|c|c|c|c|c|c|c|c|}
\hline & \multirow[b]{2}{*}{$d f$} & \multicolumn{2}{|c|}{ Pretest } & \multicolumn{2}{|c|}{ Pasttesta } & \multicolumn{2}{|c|}{ FU-1a } & \multicolumn{2}{|c|}{$F U^{2} 2^{a}$} \\
\hline & & $F$ & $p$ & $\mathrm{~F}$ & $p$ & $F$ & $p$ & $\mathbb{F}$ & $p$ \\
\hline Multivariate F Wilks & $6,24 b$ & 0.96 & .47 & 0.55 & .76 & 1.26 & .31 & 0.94 & .48 \\
\hline FQ maln complaint & $1,29 \mathrm{c}$ & 0.20 & .66 & 1.17 & .29 & 0.97 & .33 & 0.03 & .86 \\
\hline FO agoraphobia & 1,29 & 2.88 & 10 & 0.09 & .76 & 0.39 & .54 & 1.79 & .28 \\
\hline Fearl of fear & 1,29 & 2.13 & .16 & 0.49 & .49 & 0.34 & .57 & 1.62 & .21 \\
\hline STAl sitate & 1,29 & 3.11 & .09 & 0.35 & .56 & 1.67 & .21 & 0.88 & .36 \\
\hline STAI tráit & 1.29 & 5.13 & .03 & 0,06 & $.8 \%$ & 0.07 & .79 & 0.03 & .88 \\
\hline $\mathrm{BDr}$ & 1,29 & 3.98 & .056 & 0.48 & .49 & 3.39 & .08 & 0.93 & .34 \\
\hline Paric frequency & 1,27 & 0.61 & .44 & 0.33 & .57 & - & - & - & - \\
\hline
\end{tabular}

\footnotetext{
a Scores are change scores indicating the amount of improwement

b At postlest: $d f=6,22$ (interaction effect was included in analysis)

c At posttest: of $=1,27$ (interaction effect was included in analysis)
}

\section{Regression Analyses}

To further examine the contribution of personality pathology to the outcome of treatment, a series of regression analy ses was conducted using a composite measure of all outcome variables as the criterion variable. The composite score was defined as the sum of the Z-scores of the outcome variables. Four sets of analyses were conducted, one for pretest scores, one for pre-post change, one for pre-Fu-1 change, and one for pre-Fu-2 change. First, analysis was conducted with the total number of personality traits as predictor. Second, stepwise regression analyses were conducted on personality traits of specific PDs. The alpha to enter was set at .05 . In the analysis of change scores, the pretest composite score was entered in the equation first, to control for its contribution to outcome.

At pretest, the total number of personality traits predicted higher initial level of symptomatology $[B=0.43, t(1,29)=2.59, p=.02]$. At posttest, FU-1 and FU-2, the results confirmed the previous findings: the total number of personality traits was not related to the outcome of treatment $[|\mathrm{Bs}| \leq 0.15, \mid$ is $\mid(2,28) \leq .71, p \mathrm{~s} \geq$
$.48]$.

At pretest, the stepwise regression analysis revealed a significant contribution to higher initial levels of symptomatology only for avoidant personality traits $[B$ $=0.43, f(1,29)=2.59, p=.02$ ]. At posttest, FU- 1 and $\mathrm{FU}-2$, only obsessive compulsive traits contributed significantly to change. Obsessive compulsive traits predicted less improvement [posttest, $B=0.37, I(2,28)=2.06, p=.049 ; \mathrm{FU}-1, B=0.41, t(2,28)$ $=2.37, p=.03 ; \mathrm{FU}-2, \mathrm{~B}=0.45, t(3,27)=3.03, p=.01]$. Surprisingly, borderline traits predicted more improvement at $\mathrm{FU}-2[=$ change from pretest to FU- $2 ; B=-0.50, t(3$, $27)=-3.32, p=.002]$. 
1.1 Fear Questionnaire main complaint

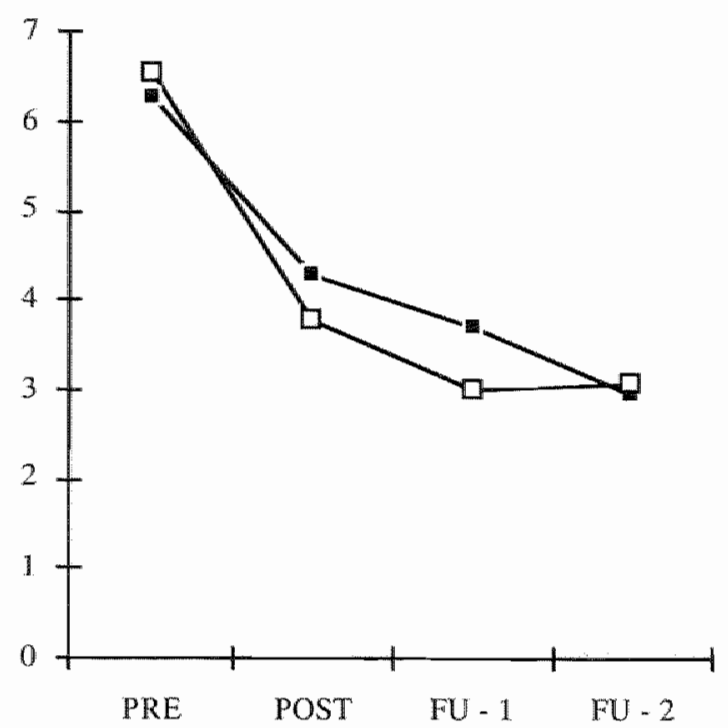

1.2 Fear Questionnaire agoraphobia

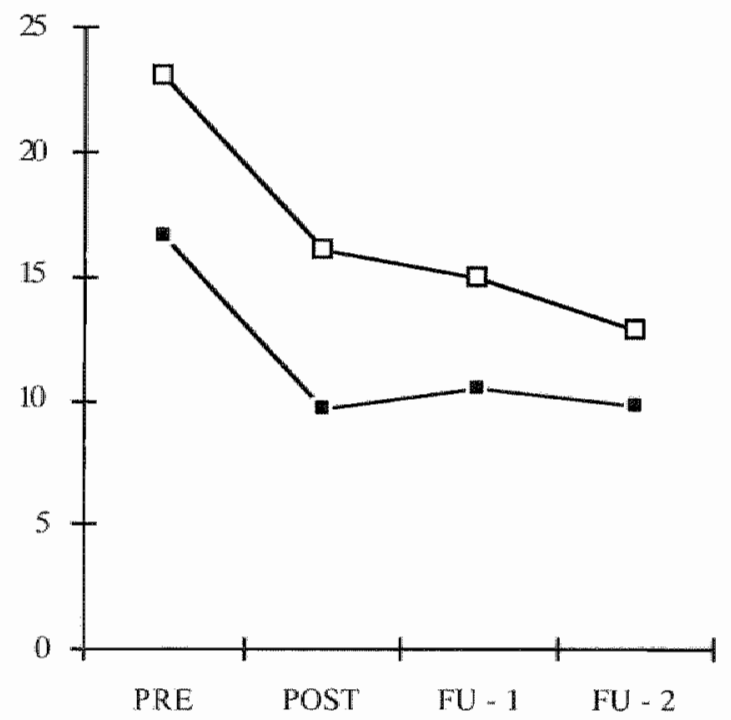

FIGURE 1

Study 1: Mean ratings before (PRE) and after the standardized treatment (POST) and at FU.1 and FUI-2 of patients with a PD (D) and without a PD (1) 


\subsection{Fear of Fear}

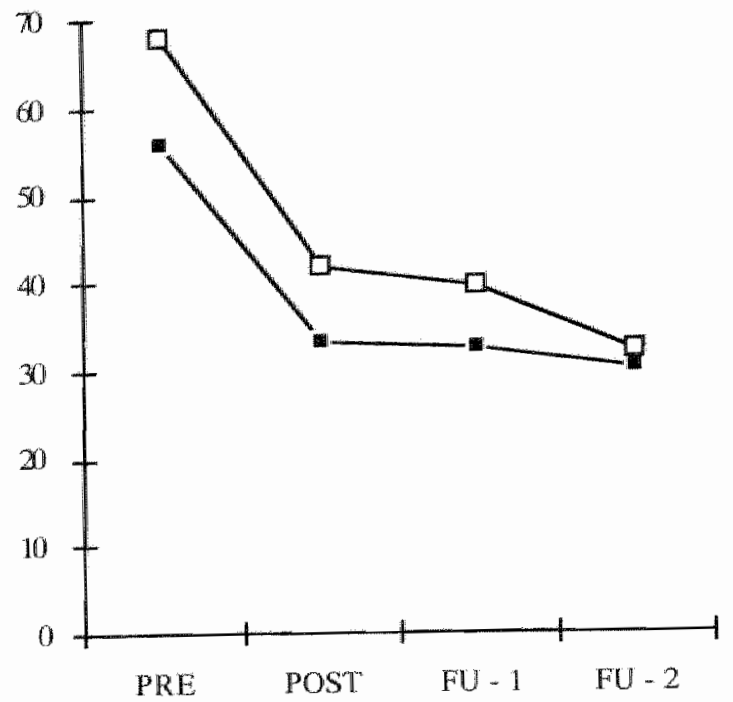

1.4 STAl state anxilety

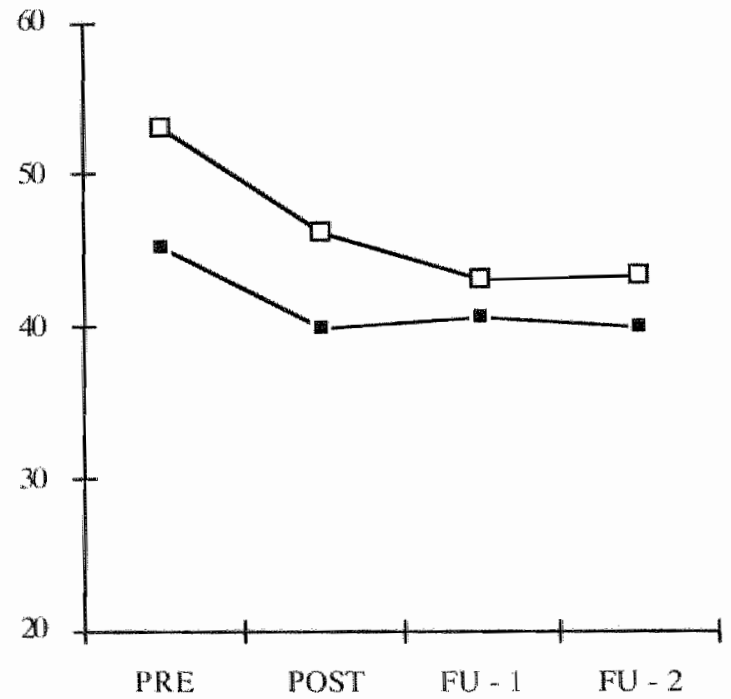

FlGURE 1 (continued)

Study 1 : Mean ratings before (PRE) and after the standardized treatment (POST) and at FU-1 and FU-2 of patients with a PD (D) and without a PD (4) 


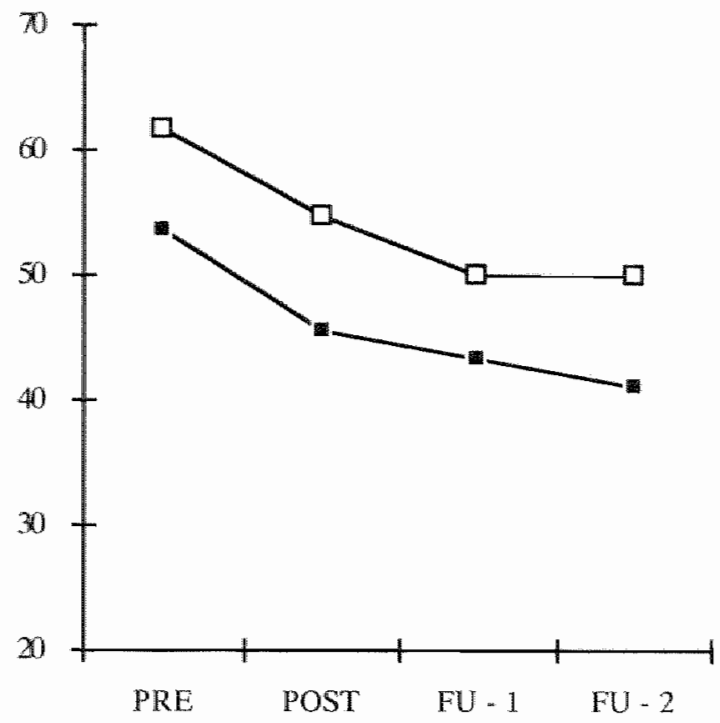

\subsection{Beck Depression Inventory}

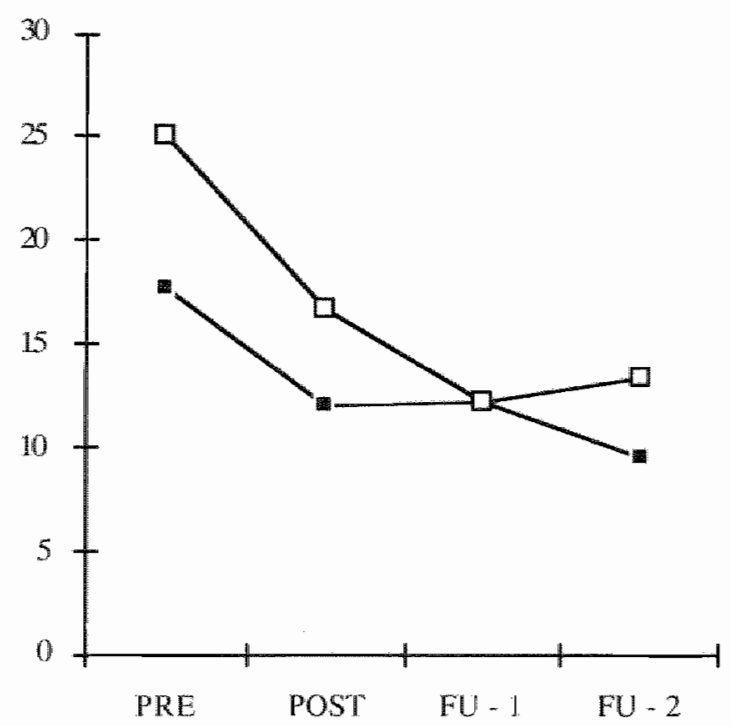

FIGURE 1 (continued)

Study 7: Mean ratings before (PRE) and after the standardized treatment (POST) and at FU 1 and FU-2 of patients with a PD (D) and without a PD (薎) 


\subsection{Mean number of PAs per day}

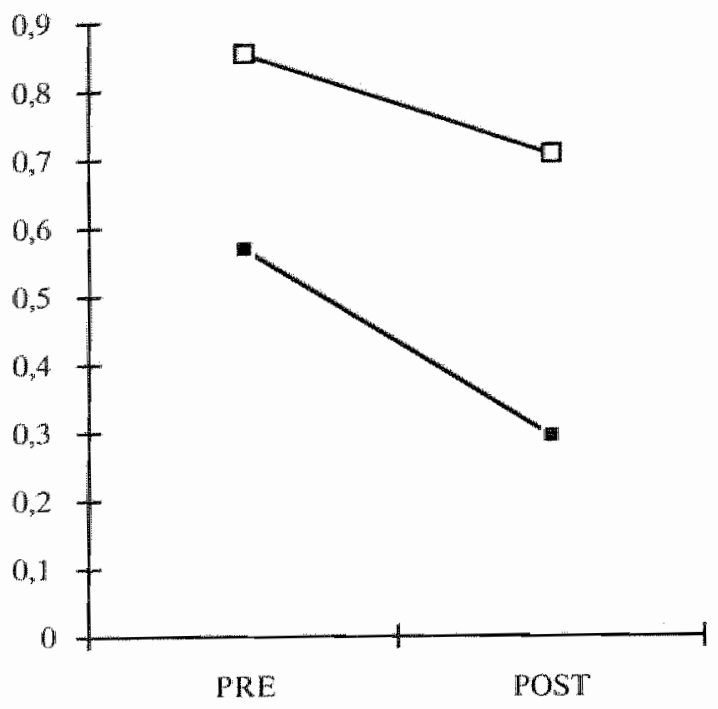

FIGURE 1 (continued)

Study 1: Mean ratings before (PRE) and after the standardized treatment (POST) and at FU-1 and FU-2 of patients with a PD ( $\square$ ) and without a PD (10)

\section{Dropouts}

Four patients dropped out of treatment before the posttest, resulting in the number of 31 participants mentioned above. Three ended treatment early without significant relief of their treatment complaints (clinical judgment). Of intenest is the presence of PDs in the subgroup of drop-outs. There is some reason to assume that patients with PDs tend to select themselves out of treatment (Reich, 1988). Because of this, a research study might miss a significant effect of PD. As to the four dropouts, only one received a PD diagnosis. This patient ended treatment early but successfully. According to the present data, there is no reason to suspect a selection bias for PD. 


\section{Study two}

The influence of PDS on individually tailored cognitive behavioral treatment for various anxiety disorders

\section{Method}

\section{Participants}

Participants were 57 patients who had been referred for cognitive behavioral treatment at the 'Academic Section Behavior Therapy' of the Community Mental Health Center for outpatients at Maastricht. All of the patients met the criteria for a DSM-III-R anxiety disorder (APA, 1987) based on an unstructured interview (23 panic disorder with agoraphobia, seven panic disorder without agoraphobia, 10 social phobia, one simple phobia, six obsessive compulsive disorder, seven generalized anxiety disorder, and three atypical anxiety disorders). According to the SCID-ll interview, 31 patients had no PD and 26 had one $(n=15)$ or more $(n=11)$ PDS. Of the patients with one PD, six were diagnosed as dependent, four as avoidant, three as paranoid, one as histrionic, and one as obsessive compulsive. Among the patients with more than one $\mathrm{PD}$, there were eight avoidant, seven dependent, six obsessive compulsive, five paranoid, two passive aggressive, one schizotypal, one borderline, one histrionic, one narcissistic, and one self-defeating PD(s).

In the non-PD group, $45.2 \%$ were male, $32.3 \%$ were married or living with a partner, and $77.4 \%$ were employed. Their mean age was $34.3 \pm 8.5$ years, and the mean duration of the Axis I illness was $6.9 \pm 6.4$ years. In the $P D$ group, $38.5 \%$ were male, $19.2 \%$ were married or living with a partner, and $73.1 \%$ were employed. Their mean age was $38.5 \pm 11.1$ years, and the mean duration of the Axis I illness was $7.5 \pm 7.7$ years. There were no differences between groups on any of the demographic variables ( $p s>10$ ).

\section{Assessment Instruments}

As in study 1, a Dutch version of the SCID-II (Spitzer et al., 1990) was used to diagnose the PDS. Six treatment outcome measures were used in the present study. Of the $\mathrm{FQ}$ (Marks Matthews, 1979), three subscales were analyzed: awoidance of the main complaint, agoraphobia, and social phobic avoidance. The 90-item Symptom Checklist (SCL-90; Arrindell \& Ettema, 1981) measured general psychopathology. Depression ratings were obtained by the Depressive Symptom Inventory (Bouman, 1987), a Dutch parallel test of the BDi. Of the Eysenck Personality Questionnaire (EPQ; Sanderman, Eysenck, \& Arrindell, 1993), the neuroticism scale was analyzed.

\section{Procedrute}

The therapist individually tailored treatment to the patient, but only used cognitive and behavioral techniques. Treatment consisted of 24.6 one hour sessions, on average $(S D=11.4)$. The mean duration of treatment was 10.8 months $(S D=5.5) . A s$ in study 1 , the therapists and patients were not given any information as to the PD diagnosed by the SCID-II. Before the start of the individually tailored treatment and immediately following treatment, patients completed the battery of self-report questionnaires. 


\section{Resullts}

Data were analyzed with multivariate analyses of variance. As in study 1 , treatment outcome was investigated as the change with respect to the pretest scores.

Effects of any po

Table 2 presents the multivariate (Wilks) and univariate $F$ statistics for all questionnaires; alpha was set at .05 for all (two-tailed) tests. Figure 2 shows the mean ratings before and at the end of treatment.

At pretest, patients with $\mathrm{PD}(\mathrm{s})$ had significantly higher ratings on social phobic avoidance (FQ), general psychopathology (SCL-90), depression (Depressive Symptom Inventory), and neuroticism (EPQ) compared with patients without a PD (Table 2). The general psychopathology and depression scores even reached Bonferroni-corrected levels of significance (corrected alpha $=.008$ ). The multivariate F statistic was significant (Table 2).

At posttest, patients with $\mathrm{PD}(\mathrm{s})$ were improved in parallel to patients without a $\mathrm{PD}$ (Table 2, univariate F statistics), except for the SCL-90 score, on which they improved significantly more. This effect did not reach a Bonferroni-corrected level of significance; however, the multivariate $\mathrm{F}$ statistic was significant (Table 2). Patients with and without $\mathrm{PD}(\mathrm{s})$ did not differ in the total number of treatment sessions $[t(1,55)=1.12, p=.27]$ and the total duration of treatment [in months, $t$ $(1,55)=0.98, p=.33]$.

In short, patients with any anxiety disorder and a concomitant PD have higher scores on some of the outcome measures at the start of therapy. The multivariate test indicates that the more severe initial psychopathology of patients with $\mathrm{PD}(\mathrm{s})$ is not a chance finding. However, these patients benefit equally or even more from treatment focused on the anxiety disorder.

TABLE 2

Study 2: Effects of PD on pretest and treatment outcome of individually tailored cognitive behavional therapy of anxiety disorders

\begin{tabular}{|c|c|c|c|c|c|}
\hline \multirow[b]{2}{*}{ Unhariate trests } & \multirow[b]{2}{*}{$n$} & \multicolumn{2}{|c|}{ Pretest } & \multicolumn{2}{|c|}{ Pastitesta } \\
\hline & & $t$ & $p$ & $t$ & $p$ \\
\hline FO main complaint & 57 & 0.31 & .76 & 0.18 & .86 \\
\hline FO agoraphobia & 57 & 0.40 & .69 & 0.47 & .64 \\
\hline $\mathrm{FQ}$ social phobia & 56 & 2.62 & .01 & 0.45 & .66 \\
\hline $\begin{array}{l}\text { SCL } 90 \text { general } \\
\text { psychopathology }\end{array}$ & 57 & 425 & $<001$ & -2.13 & .04 \\
\hline DSI & 57 & 4.10 & $<.001$ & -4.59 & .12 \\
\hline EPQ-N & 56 & 2.23 & .02 & 1.56 & .13 \\
\hline Mulgivariate F Wilks & dt 6,49 & $F=4.29$ & $p<.001$ & $F=3.15$ & $p=.01$ \\
\hline
\end{tabular}

DSI $=$ Depressive Symptom Inventory

a Postest scores are chainge scores indicating the amount of improvement 
2.1 Fear Questionnaire main complaint

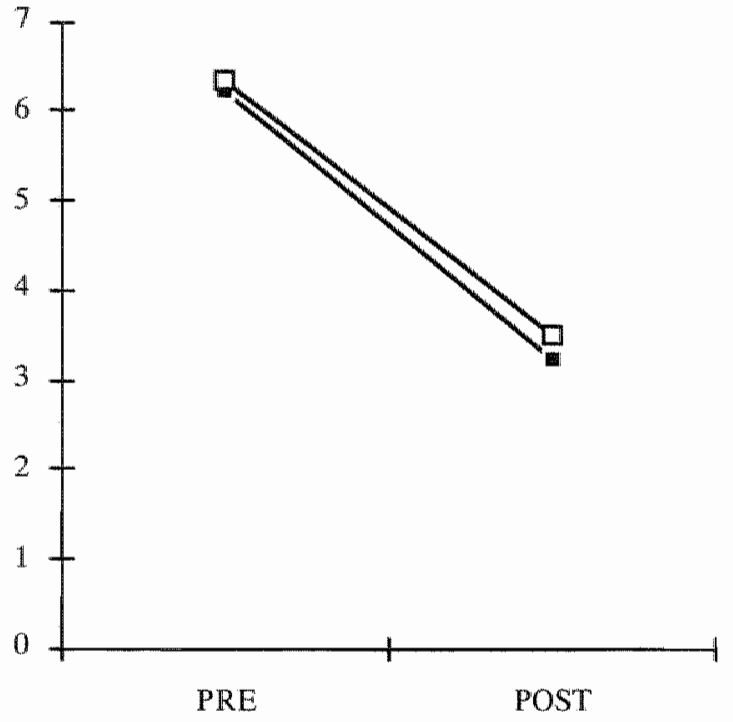

\subsection{Fear Questionnaire agoraphobiia}

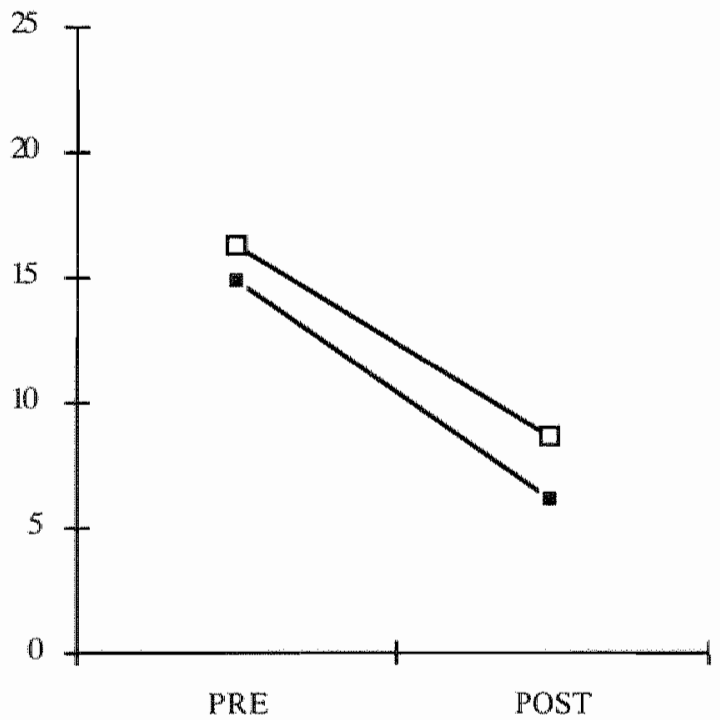

FIGURE 2

Study 2: Mean ratings before (PRE) and after (POST) the individually tailored treatment of patients with a PD (D) and without a PD ( 
2.3 Fear Questionnaire social phobia

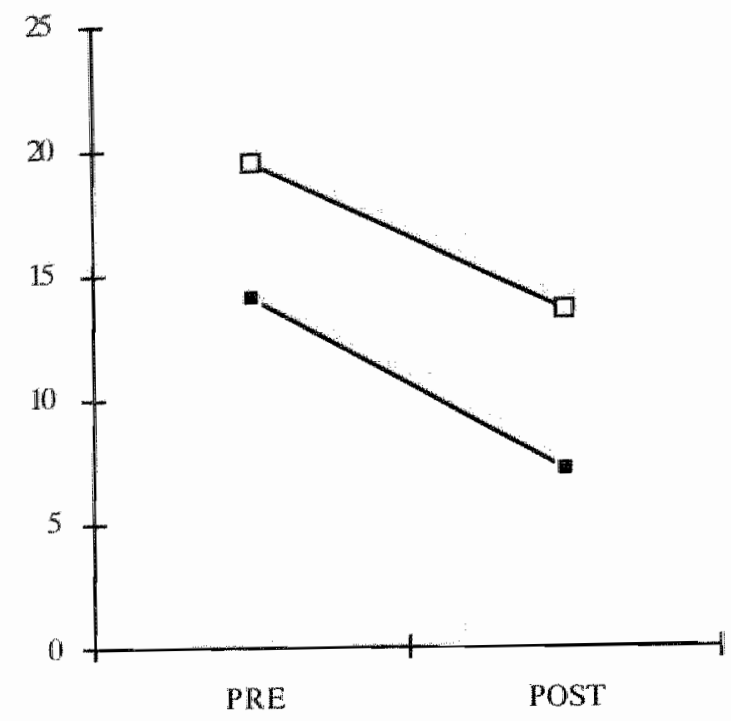

2.4 SCL-90 general psychopathology

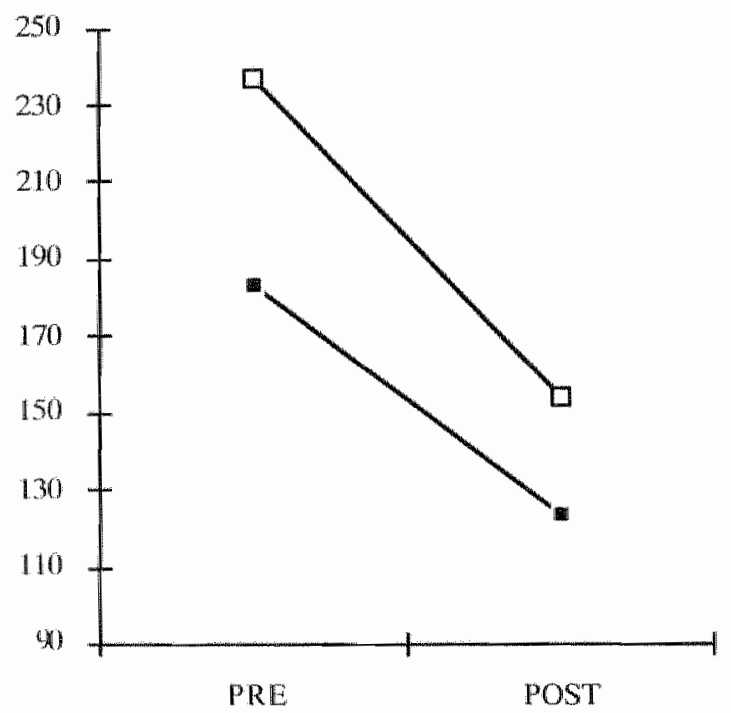

FIGURE 2 (continued)

Study 2: Mean ratings before (PRE) and after (POST) the individually tailored treatment of patients with a $\mathrm{PD}(\mathrm{D})$ and without a $\mathrm{PD}(\mathbf{1})$ 
2.5 Depressive Symptom Inventory

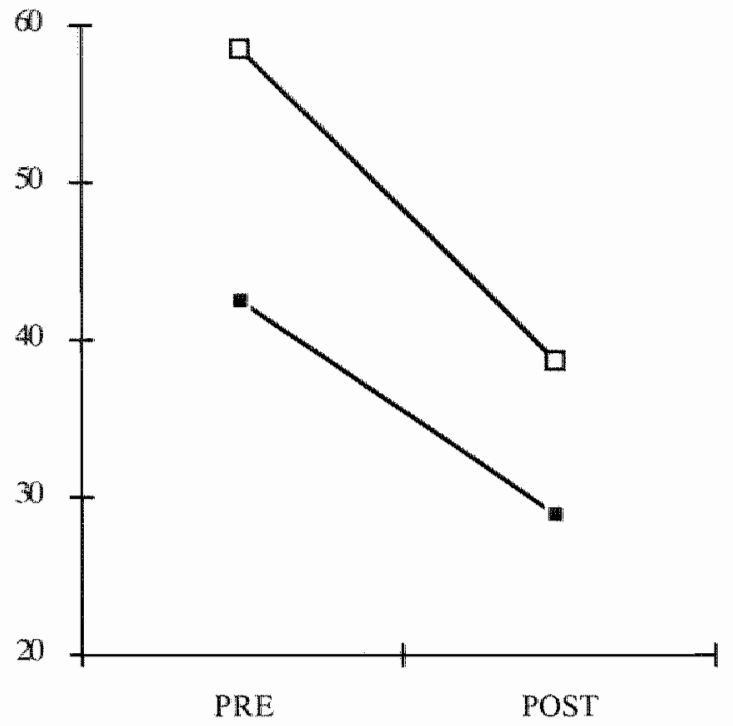

\subsection{EPQ neuroticism}

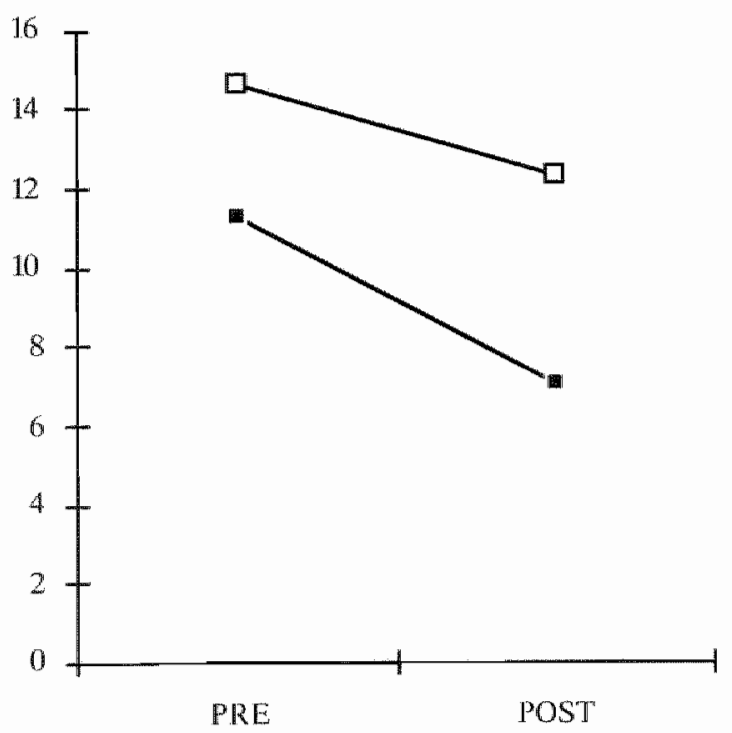

FIGURE 2 (continued)

Study 2: Mean ratings before (PRE) and after (POST) the individually tailored treatment of patients with a PD ( $\square)$ and without a PD (1) 


\section{Regression Analyses}

Regression analyses were conducted on pretest scores and pre-post change, using the composite measure of all outcome variables as the criterion variable. In a first analysis, the predictor variable entered was the total number of personality traits received. Second, stepwise regression analyses were conducted on personality traits of specific PDs. The alpha to enter was set at .05 . In analyses of change scores, the pretest composite score was entered in the equation first to control for its contribution to outcome.

At pretest, the total number of personality traits predicted higher initial levels of symptomatology $[B=0.62, t(1,55)=5.78, p<.001]$. At posttest, the total number of personality traits was not related to the outcome of treatment $\llbracket \beta=0.08, t(2,54)$ $=.03, p=.64]$.

At pretest, the stepwise regression analysis on personality traits of specific PDs showed a significant contribution to higher initial levels of symptomatology for dependent $[B=0.28, t(3,53)=2.18, p=.03]$, obsessive compulsive $[B=0.26, t(3$, $53)=2.27, p=.03]$, and borderline personality traits $[B=0.36, t(3,53)=3.17, p=$ $.003]$. At posttest, only obsessive compulsive traits predicted less improvement [ $B$ $=0.36, t(2,54)=2.69, p=.009]$.

\section{Drop-outs}

Sixteen patients dropped out of the study before the posttest, resulting in the number of 57 participants mentioned earlier. A matter of interest is whether there is a tendency for patients with $\mathrm{PD}(\mathrm{s})$ to drop out of treatment or to refuse to com-

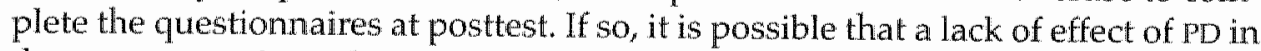
the present study is due to a selection bias. Comparisons between participants who did and did not provide a complete data set indicated that there were no differences in the presence of PDs $\left[\operatorname{chi}^{2}(1, n=72)=0.09, p=.77\right]$. In the drop-out group, $50 \%$ of the patients had one or more $\mathrm{PD}(\mathrm{s})$. In the sample that completed the posttest, $46 \%$ of the patients had one or more PD(s). Moreover, $t$-tests revealed that for each separate PD category, there is no difference in the number of personality traits between the group included in analyses and the drop-out group $[\mid$ ts $\mid(1,55)$ $\leq 1.18, p s \geq .24]$.

\section{Discussion}

Study 1, including only panic patients, showed some evidence for increased psychopathology with PD panic patients at the start of treatment. Panic patients with and without PDs seemed to profit equally from cognitive behavioral treatment focused on the panic disorder. Study 2, including several types of anxiety patients, demonstrated that anxiety patients with $\mathrm{PD}(\mathrm{s})$ had more generalized problems at the start of treatment than patients without PD. They improved in parallel to the patients without PD, so they still had higher levels of problems after treatment. The findings of the present two studies are hopeful and encouraging; patients with PDS can be helped by cognitive behavior therapy for their anxiety disorders and do not seem to profit less from treatment than patients without PDs. This conclusion goes against expectations generated by other studies (Chambless et al., 1992; Farvelli \& Albanesi, 1987; Green \& Curtis, 1988; Hermesh et al., 1987; 
Kringlen, 1965; Lo, 1967; Mavissakalian \& Hamann, 1987; Minichiello et al., 1987; Numberg et al., 1989; Rabavilas et al., 1979; Reich, 1988; Reich, 1990; Turner, 1987; Tyrer et al., 1983; Vaughan \& Beech, 1985). In addition to the issues of type of treatment, type of Axis I disorder and type of PD, the critical remarks on methodological differences that were addressed in the beginning of this report might explain some variation in findings between previous studies and the present studies. The present studies used a clinical semistructured interview in combination with a prospective double-blind design, validated measurements, and change scores as quantification of improvement. Further studies are needed to explore the possible reasons for divergence in findings.

As a comment on the two presented studies, several issues should be noted, which might also be interesting points in explaining the divergence in findings. First, the conclusion of the two presented studies considers the influence of PDS in general, irrespective of type. Lumping PDs together was used as a strategy to obtain adequate sample sizes, but is based on the assumption that any PD will have the same effect on cognitive behavioral treatment of anxiety disorders. Hitherto, this assumption is far from being properly tested. Previous studies showed positive, negative, and no influences of different PDs on the treatment of various anxiety disorders (Chambless et al., 1992; Green \& Curtis, 1988; Hermesh et al., 1987; Kringlen, 1965; Lo, 1967; Minichiello et al., 1987; Numberg et al., 1989; Rabavilas et al., 1979; Reich, 1988; Reich, 1990; Steketee, 1990; Vaughan \& Beech, 1985). Study 2 provided data for post hoc analyses on four individual PDs (paranoid PD, $n=9$; avoidant PD: $n=12$; dependent $\mathrm{PD}, n=13$; and obsessive compulsive $\mathrm{PD}, n=7$ ), and none of these individual PDs were a consistent predictor of poorer or better outcome (six outcome measures: paranoid PD was no predictor of improvement, avoidant PD predicted more improvement on one measure, dependent PD predicted less improvement on one measure, and obsessive compulsive PD predicted less improvement on two measures. All effects failed to reach the Bonferroni-corrected level of significance).

The present studies investigated the influence of the number of personality traits of specific PDS. It might be postulated that personality traits already influence treatment outcome before the minimum set of traits for a PD is present. However, the total number of personality traits, irrespective of type, was unrelated to amount of improvement. As to the traitscores of specific PDs, only obsessive compulsive traits were a consequent predictor of less improvement. This finding should be interpreted with caution because of the large number of tests that were done. Replication is needed. In study 1, borderline personality traits predicted better outcome only at FU-2. First of all, it should be noted that borderline traits are not equivalent to a borderline PD. Two reasons for this rather surprising effect were considered, but not proven assumable. First, the characteristic dichotomous thinking in borderline personalities would suggest overevaluation of progress, but then one would expect the same effect at posttest and at FU-1, which was not the case. Second, additional treatment after FU-1 might have caused additional progress. However, borderline traits were not positively correlated with additional treatment sessions $(r=.10, p=.96)$.

In conclusion, there are only limited indications from the present studies that individual PDs have differential effects on cognitive behavioral treatment of anxiety 
disorders. However, it should be noted that the more severe PDs of clusters A and $B$ were infrequently diagnosed in the present studies (schizotypal, schizoid, antisocial, borderline and narcissistic), which brings on a second point of commentary.

The differences in prevalence of individual PDS might be a consequence of the type of Axis I disorders selected for study. Because the more severe types of PDS of clusters A and B were infrequently diagnosed, results of the present studies may be restricted to the more frequently diagnosed PDs (avoidant, dependent, paranoid and obsessive compulsive). Several previous studies (Hermesh et al., 1987; Minichiello et al., 1987; Numberg et al., 1989; Reich, 1988 Reich, 1990) specifically examined PDS of cluster A or B and reported negative effects on treatment outcome. Therefore, the low prevalence of cluster A and B PDs might have contributed to the lack of a negative effect of any PD on treatment outcome. However, there is also evidence that the more severe PDs of cluster A and B do not affect treatment outcome (Chambless et al., 1992; Steketee, 1990).

A third point of commentary is that one could criticize the fact that there was no effect in the study regarding panic patients because of the small sample size. However, there is not even a trend towards a negative influence of $P D$ on the efficacy of cognitive behavioral treatment of panic disorder. So, larger sample sizes are unlikely to reveal a negative effect.

Fourth, the severity of any PD might be an important factor in studying PDs. A $P D$ is present when at least the specified number of criteria is met. If present, the current severity of a PD can be expressed as mild, moderate or severe (which is related to the quantity and quality of the personality traits). Possibly, there is a critical level of severity necessary to affect treatment outcome, even if a PD is present according the number of criteria met. This was not studied in the present studies. On the other hand, the critical severity of a PD that is related to treatment efficacy might be at what can be called the subthreshold level, which means that the specified number of criteria for diagnosing a PD is nearly present. If this is true, patients with subthreshold PDs that are now classified in the non-PD group might influence treatment outcome as much as PD patients in the PD group, with the consequence that the two groups show no differences. However, results from the regression analyses do not provide evidence for this hypothesis, with the exception of obsessive compulsive personality traits. These traits should be looked at more closely in further research.

Fifth, the present studies investigated cognitive behavioral treatments. Possibly, cognitive behavior therapy is more suitable for patients with PDs than other treatments, for instance, pharmacological treatments. Of four previous studies on only pharmacotherapy, three reported negative results (Green \& Curtis, 1988; Reich, 1988; Tyrer et al., 1983) and one study reported no effect of PDs (Mavissakalian et
al., 1990).

A final point of commentary is that the anxiety patients with PD still manifested rather high levels of general psychopathology complaints and neuroticism after therapy (end-state functioning), which suggests that some complaints related to their $\mathrm{PD}$ were not affected by the treatment that was focused on the anxiety disorder. This might constitute a risk for later relapse. However, the positive reaction of the patients with PDS to treatment that was focused on the anxiety disorder 
suggests that additional treatment specifically focused on problems directly related to the PD might result in even more positive effects. Although this is undoubtedly a more difficult enterprise than the treatment of Axis I disorders (Arntz, 1993; Beck, Freeman, \& Associates, 1990; Padesky, 1988; Pretzer \& Fleming, 1989; Turkat, 1985), and new strategies have to be developed, the present findings, indicating that patients with PDs can profit from oognitive behavior therapy, are promising. In conclusion, taking into account some limitations, the results of this investigation indicate that PDs do not predict poorer outcome of cognitive behavior therapy of Axis I anxiety disorders in general and panic disorder in particular. One should be careful in using $\mathrm{PD}$ as an exclusion criterion in cognitive behavior therapy for anxiety disorders, and a lack of progress should not be too easily attributed to a PD. 


\section{notes}

1 Published as: Dreessen, L., Arrtz, A, Luttels, C, k Sallaerts, S. (1994). Personality disorders do not influence the results of cognitive behavior therapies for anxiety disorders. Conprehensize Psychiatry, 35, 265-274.

2 Section 3.1 was written in 1993. Consequently the introduction is not completely current. At present, more than 17 studies have addressed the issue of the influence of $\mathrm{PD}(\mathrm{s})$ on the effects of psychotherapeutic and/or pharmacological treatment of anxiety disorders (see Chapter 4 of the present thesis). 


\section{Personality disorders do not influence the results of cognitive and behavior therapy for obsessive compulsive disorder ${ }^{1}$}

\section{Summary}

This study examined whether categorical or dimensional personality disorder variables affected treatment outcome in a sample of 52 patients with obsessive compulsive disorder who followed a standardized cognitive behavior therapy program. Treatment consisted of 12 weekly sessions and was completed by 43 patients. The Structured Clinical Interwiew for DSM-nil$\mathrm{R}$ Personality Disorders (SCD-1) was taken before the start of treatment by an independent rater. The treatment outcome measures included questionnaires and a Behavioral Assessment Test. Measurements were taken before and after treatment, and at 1 and 6 month follow-ap tests. After the first followup test, further treatment was provided if clinically indicated. Neither categorical, nor dimensional personality disorder variables affected treatment outcome significantly. The inclusion of drop-outs in the analyses, did not change these results. Therefore, patients with obsessive compulsive disorcler and concomitant personality disorder pathology should not be excluded from cognitive or behavior therapy for their obsessive compulsive complaints. Attributing therapy failure to concomitant Axis II pathology should be approached with caution.

\section{Introduction}

Clinicians often presume that the treatment of Axis I disorders (American Psychiatric Association, 1994) is complicated in patients with a concomitant personality disorder (PD). Moreover, therapeutic failure is easily attributed to a concomitant PD. With regard to behavior therapy for obsessive compulsive disorder (OCD; Axis I), five studies were traced that focused on the prognostic influence of concomitant PDs (Fals-Stewart \& Lucente, 1993; Minichiello, Bear, \& Jenike, 1987; Rabavilas, Boulougouris, Perissaki, \& Stefanis, 1979; Steketee, 1990; Vaughan \& Beech, 1985). Evidence for a negative impact of some PDs on treatment outcome of OCD was found in three studies. Minichiello et al. (1987) reported a negative effect of schizotypal PD on immediate outcome. Fals-Stewart and Lucente (1993) reported that the cluster of schizoid, avoidant, dependent and schizotypal traits was related negatively to immediate and longer-term treatment outcome. The cluster of histrionic and borderline traits was related negatively only to longer-term 
treatment outcome. Steketee (1990) found that passive aggressive traits were associated with more relapse. In contrast to some of the above findings, previous studies also reported nonsignificant or positive relationships between PD variables and OCD treatment outcome. Fals-Stewart and Lucente (1993) found that the cluster of dependent and obsessive compulsive traits was unrelated to OCD treatment outcome. Steketee (1990) found that schizotypal, histrionic, avoidant, and dependent PD were unrelated to immediate and longer-term treatment outcome. Avoidant and dependent traitscores were even correlated positively to treatment outcome. The presence of one or more PDs, regardless of type, was also unrelated to OCD treatment outcome. Regarding obsessional personality, Rabavilas et al. (1979) and Vaughan and Beech (1985) found evidence for a positive impact on treatment outcome of OCD. In short, in previous studies, PD variables were sometimes found to be assiociated negatively with ocD treatment outcome, but not consistently throughout the different studies.

Several methodological issues concerning the above studies warrant discussion. First, none of them used a (semi)structured interview for the assessment of personality pathology. In general, (semi) structured interviews are believed to be more valid than unstructured interviews and self-report questionnaires (Dreessen \& Arntz, 1998-a; Widiger \& Frances, 1987; Zimmerman, 1994).

Second, in two studies (Minichiello et al., 1987; Vaughan \& Beech, 1985) personality pathology was assessed after treatment. If therapy with a particular patient is known to be a failure, it might cause the rater to diagnose more Axis II pathology than if the rater is blind to the treatment results.

Third, only one study (Steketee, 1990) used a pre-posttest design, which we think is preferable to studying improvement in terms of end-state functioning or in terms of responders and non-responders. Responders have to show minimal symptomatology on all outcome measures. Patients with concomitant PD often have higher pretest scores than those without a PD (e.g. Dreessen, Arntz, Luttels, \& Sallaerts, 1994). In that case, patients with PD can have higher symptom scores after treatment, but compared to pretest scores, they might have improved parallel to patients without PD. Quantifying improvement in terms of end-state functioning or in terms of responders might obscure parallel improvement, which can be demonstrated in a pre-posttest design. Given the inconsistency of the results and the methodological shortcomings of previous studies, further studies into the impact of PDs on treatment outcome of cognitive and behavior therapy of ocD are
needed.

The present study was designed to study the influence of po variables on treatment outcome following a standardized cognitive and/or behavior therapy (CBT) program for patients with OCD. In contrast to previous studies, PDs were assessed prior to the treatment by means of a semi-structured interview for DSMIII-R (American Psychiatric Association, 1987) PDs and improvement was studied
in terms of change with respect to pretest Scores. 


\section{Method}

\section{Patients}

The participants were 52 patients who met the DSM-111-R criteria for OCD (APA, 1987). Patients were referred by mental health professionals to the "Academic Section Behavior Therapy' of the Community Mental Health Center for outpatients at Maastricht. Axis I diagnoses were assigned following administration of the Structured Clinical Interview for DSM-III-R (SCDD; Spitzer, Williams, \& Gibbon, 1987). The SCID was administered by experienced cognitive behavioral therapists, all of whom were trained in using the SCID. In all patients, the SCID-interviewer considered $O C D$ to be the main complaint. In addition, 12 patients had a concomitant depressive disorder, 5 had a social phobia, 5 had a panic disorder with or without agoraphobia, 3 had a generalized anxiety disorder, 1 had a simple phobia, 3 had a somatoform disorder, and 1 had a psychotic disorder NOS. Moreover, according to the unstructured clinical interview, two patients had a sexual dysfunction, two had a phase of life problem, one had a marital problem and one had a parent-child problem (DSM-diagnoses, APA, 1987).

Forty-three patients completed the OCD treatment protocol. The remaining nine patients dropped out of treatment for various reasons. Patients were considered as completers if they finished 12 therapy sessions scheduled once a week without starting any other treatment during that time (including pharmacotherapy). If patients occasionally missed an appointment, the 12-wreek treatment interval was prolonged by a couple of weeks, without giving the patients more than 12 sessions.

Axis In diagnoses were assigned following administration of the Structured Clinical Interview for DSM-III-R Personality Disorders (SCID-II; Spitzer, Williams, Gibbon, \& First, 1990). Of the completers, 14 patients had no PD, 7 had only a subthreshold diagnosis, and 22 had one $(n=13)$ or more $(n=9)$ PDs. Subthreshold diagnoses were made if the patient fulfilled the minimum number of traits minus one required by the DSM-III-R. Of the drop-outs, two patients had no IPD and seven had one $(n=5)$ or more $(n=2)$ full-blown PDs. There were no drop-outs with merely subthreshold diagnoses. Prevalence rates of specific PDs and the mean traitscores, both for completers and drop-outs, are shown in Table 1. There were no statistically significant differences (two-tailed tests) between completers and drop-outs with regard to personality pathology. Comparisons were made with regard to total number of PDS, total number of traits, and traitscores of specific PDS ( $\left|t_{s}\right| \leq 0.97, p s \geq .36$ ) and the presence of any PD (Fisher's Exact test, $p=.27$ ).

The group of completers consisted of 16 men and 27 women. The mean age was 34.1 years (range 18-61), 60.5\% were married or living with a partner. The group of drop-outs consisted of seven men and two women. Their mean age was 38.2 years (range 26-59), 44.4\% were married or living with a partner. The duration of the $\mathrm{OCD}$ and the ratings of severity of complaints at pretest for completers and drop-outs, with and without a PD are listed in Table 2. Groups with and without a $P D$ did not statistically differ with respect to duration of the $O C D$ and severity of complaints at pretest ( $\mid$ ts $\mid \leq 1.98, p s \geq .10$ ). 
TABLE 1

Number of personality disorder (PD) diagnoses and mean traitscores of OCD patients at pretest

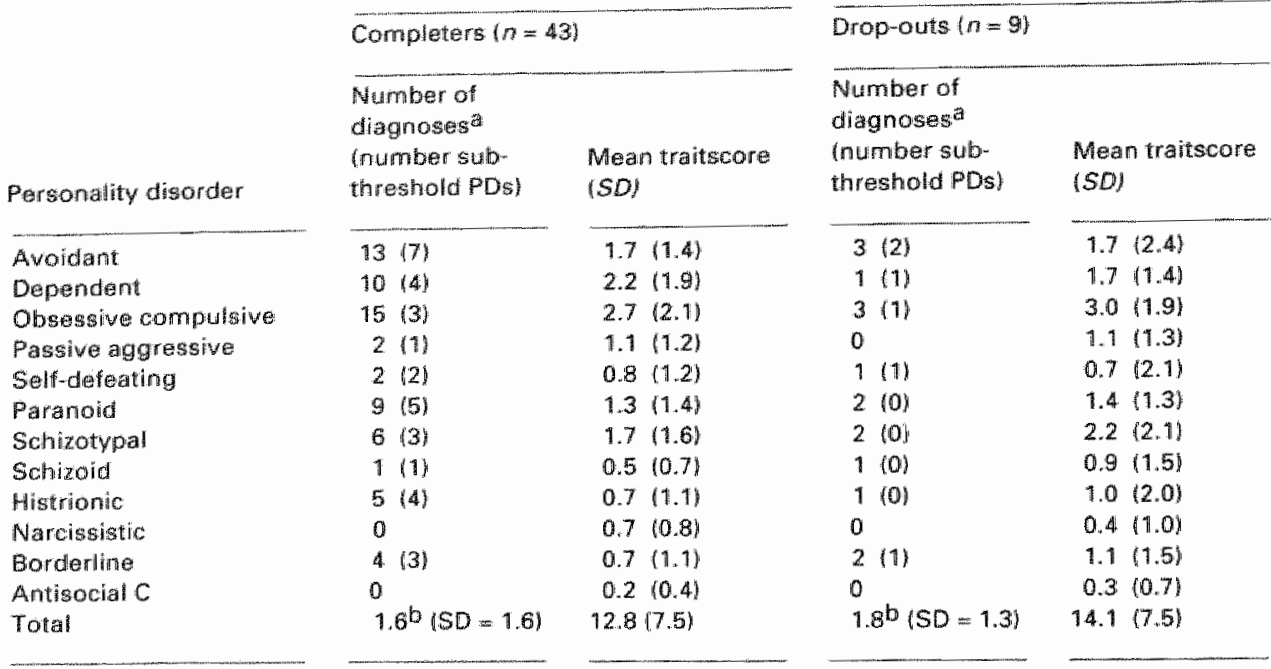

$O C D=$ Obsessive Compulsive Disorder

Antisocial $\mathrm{C}=$ antissocial behavior since the age of 15

al Subthreshald diagnoses are included

b Mean number of personality disorder diagnoses, subthreshold diagnoses included

\section{TABLE 2}

Duration of the OCD and severity of complaints at pretest of completers and drop-outs with no personality disorder (PD) and with one or more PDs

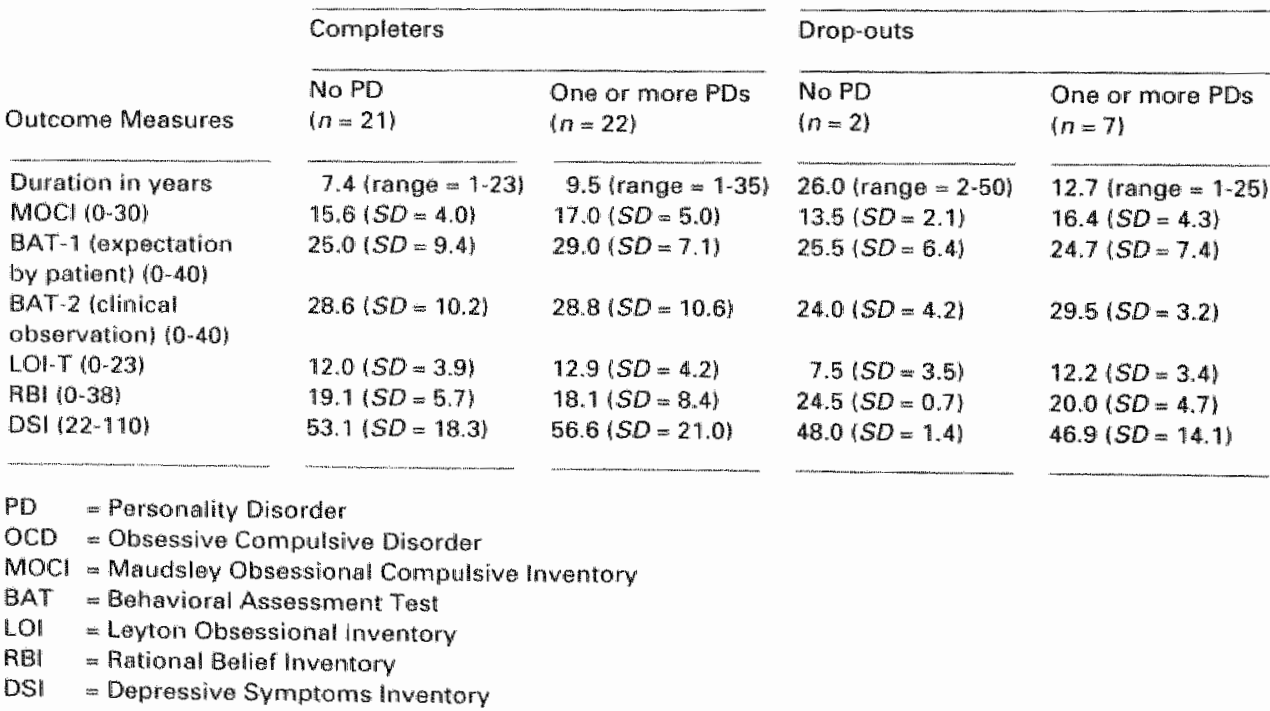


Structured Clinical Interoiew for DSM-UI-R Personality Disorders. A Dutch translation of the Structured Clinical Interview for DSM-II-R Personality Disonders (SCID-II; Spitzer et al. 1990) was used for the assessment of DSM-II-R personality traits and corresponding disorders (APA, 1987). The interviewer was instructed to discuss all SCD-1 criteria with the patient. Criteria wene rated as threshold or true (3), as subthreshold (2) or as absent (1). The joint-interview interrater reliability in our group of raters was very good on criterion level, with a few exceptions. The overall kappa was .80 (Arntz et al., 1992-b). Other studies have also found adequateto-good interrater reliability of the SCID-II using the joint-interview approach (Brooks, Baltazar, McDowell, Murjack, \& Bruns, 1991; Fydrich, Schmitz, Hennch, \& Bodem, 1996; Renneberg, Chambless, Dowdall, Fauerbach, \& Gracelly, 1992; Stanley, Turner, \& Borden, 1990; Wonderlich, Swift, Slotnick, \& Goodman, 1990). The short-interval test-retest interrater reliability has been found to be less stable and ranged from poor to good ${ }^{2}$ (First et al., 1995-b; Hennch, Lauer, \& Fydrich, 1994). The concurrent validity has been found to be modest (Hyler, Skodol, Kellman, Oldham, \& Rosnick, 1990; Hyler, Skodol, Oldham, Kellman, \& Doidge, 1992; O'Boyle \& Self, 1990; Oldham et al., 1992; Renneberg et al., 1992; Skodol, Oldham, Rosnick, Kellman, \& Hyler, 1991).

Treatment Outcome Measures. The treatment outcome measures included four self-report questionnaires and achievement on a Behavioral Assessment Test (BAT; the test was created by the second author). The Maudsley Obsessional Compulsive Inventory (MOCI; Hodgson \& Rachman, 1977) was used to measure obsessive compulsive complaints and the Leyton Obsessional Inventory Trait (LOI-T; Cooper, 1970) to measure obsessive compulsive traits. The Rational Beliefs Inventory (RBI; Shorkey \& Whiteman, 1977) was used as an index of rational thinking capacity in general. Depressive complaints were assessed with the Depressive Symptoms Inventory (DSI, Bouman, 1987), a Dutch parallel test of the Beck Depression Inventory. These questionnaires were selected because they were in common use when the study commenced in 1986 (e.g. Emmelkamp, 1982) and because Dutch translations were available. The BAT is derived from the anxiety/discomfort scale (Watson \& Marks, 1971; for a full description see: Emmelkamp, 1982) and contained five situations of increasing difficulty, taken from the personal hierarchy of the patient. These situations were described in terms of exposure with response prevention to an action the patient feared. The subjective units of discomfort of the personal hierarchy ranged from 0-100. The subjective units of discomfort of the BAT-situations were larger in value than the second quartile of the personal hierarchy. Alteast one BAT-situation had a subjective unit of discomfort higher in value than the third quartile. Patients were asked to rate their expected anxiety before performing the tests (= BAT-1 score). A research-assistant, who was blind to the BAT-1 score and who had clinical experience with OCD patients as a psychiatric nurse, rated anxiety while observing the patient performing the BAT (= BAT-2 scone). If patients failed to fully expose themselves to the situation or if they performed rituals afterwards, maximum anxiety was rated. In other words, any avoidance was rated as the maximum anxiety score. The BAT- 1 and the BAT- 2 scores correlated highly on all four assessment points $(0.59 \leq r s \leq 0.90)$. 


\section{Treannents}

Patients received either exposure in vioo with response prevention $(n=16)$, or cognitive therapy without behavioral experiments $(n=14)$, or the CBT combination $(n=22)$ for their oCD complaints [see Emmelkamp, Visser and Hoekstra (1988) for a description of the exposure in vivo therapy and the cognitive therapy]. Three different treatment techniques were applied, because this study was combined with another study that focused on the differential effects of type of treatment on therapy outcome of OCD. The results of the latter study will be submitted for publication shortly (Hoekstra, Arntz, \& Hout, van den, 1998). The type of CBI technique did not interact with the presence of one or more PDs as far as the effect on therapy outcome was concerned, $F \mathrm{~s}(2) \leq 1.87, p s \geq .17$. Treatment consisted of 1-hour individual therapy sessions scheduled once a week over a 12 -week interval. Eleven therapists ( 5 male and 6 female), all of whom were experienced in conducting CBT techniques with OCD patients, conducted the therapies. They were either officially registered (cognitive behavioral) psychotherapists, or they had reached an advanced level in their training toward becoming such a psychotherapist. The second author, trained by Emmelkamp in CBT for OCD, checked whether therapists followed the specific treatment protocols by giving weekly supervision.

Regarding the use of psychotropic medications, patients were requested either to taper them off before the start of the study or to use the same dosage during the standardized treatment program. Eventually, 11 of the patients who completed the study used a constant dosage of psychotropic medication. One patient tapered medication off before the start of the treatment and one patient changed the dosage of medication during the standardized treatment period and consequently dropped out of the study.

\section{Procedure}

Written informed consent was obtained from participating patients. The SCID-II interview was taken before the start of treatment by an independent rater. Both patients and therapists were blind to the SCDD-II diagnoses. Patients were randomly assigned to receive one of the three treatments. If clinically indicated, unstandardized cognitive and/or behavioral therapy was continued I month after completion of the treatment protocol. Patients were measured before the start of treatment and immediately after completion of the standardized treatment. Followup assessments were taken at 1 month (FU 1) and at 6 months (FU 2) after termination of the standardized treatment protocol. Groups of patients with and without $P D$ did not differ in the number of patients that received additional treatment after the FU 1 test ( $w$ ith PDS: $55 \%$, without PD: $57 \%$, chi ${ }^{2}=0.03, p=.86$ ). All patients were included in the Fu 2 analyses, regardless of whether they received additional treatment or not.

\section{Dafa Reduction and Analyses}

Treatment outcome at posttest and follow ups was investigated as the change with respect to pretest scones. Before analyzing treatment outcome, pre- to posttest change scores of patients who completed the treatment protocol, were submitted to a factor analysis in order to reduce the number of dependent variables. 
This factor analysis revealed one clear factor solution, accounting for $52 \%$ of the variance, all variables loading on it. The same held for the pretest to follow-up change scores. Results of the factor analyses allowed us to generate one composite outcome score for changes from pretest to later tests, including scores on all six outcome measures. Pre- to posttest change scores on all six outcome measures were transformed into Z-scores and added. The same was done for the pretest to follow-up change scores.

To study the impact of personality pathology on treatment outcome of $\mathrm{OCD}$, both categorical and dimensional PD variables were studied. In order to obtain adequate sample sizes with regard to specific PDS, patients with only subthreshold PDs diagnoses were also included in the PD group.

First, analyses were done with the data of patients who completed the treatment triall. Second, data of drop-outs were included in the analyses. Change scores had to be transformed into Z-scores anew, including data of the drop-outs.

Along with the composite score of all treatment outcome variables, we also examined the effect of $\mathrm{PD}$ variables on a composite score of $\mathrm{MOCI}$ and $\mathrm{BAT}$ scores. The MOCI and the BAT measured the symptoms that were targeted in the treatment and the effects of PD variables on this composite score were checked to see if they were in line with the effects on the composite of all scores.

Finally, we performed clinical significance analyses for the MOCI and the BAT scores following the method described by Jacobson and Truax (1991). Patients were classified as improved if they met a Reliable Change (RC) index $\geq 1.96$ and they were classified as recovered if they crossed the cutoff point, which was estimated as two standard deviations beyond the mean of the sample. We evaluated the effect of one or more PDs and used chi-square or Fisher's Exact tests.

\section{Results on completers (general composite score)}

Patients who completed the protocol improved significantly at the posttest and at the first and second Fu tests [ts $\leq-4.47$ [RBI-scores] and ts $\geq 3.83, \mathrm{ps} \leq .001]$.

\section{Effect of Categorical PDS}

To study the effect of categorical PDs on pretest scores and treatment outcome of $O C D$ (change with respect to pretest), two-tailed $t$ tests were done and alpha was set at .05. Five specific PDs, avoidant, dependent, obsessive compulsive, paranoid and schizoty pal PD were studied separately, because the number of positive cases was high enough ( $n \geq 6$ : subthreshold diagnoses included). Patients with one of these particular PDS were compared to all other patients without this particular PD. Results are presented in Table 3.

At the pretest, the level of complaints of patients with $\mathrm{PD}(\mathrm{s})$ did not differ significantly from patients without a $\mathrm{PD}, t(41)=1.20, p=.24$. Regarding specific PDS, patients with a dependent PD had more initial complaints than patients without a dependent $\mathrm{PD}, t(41)=2.11, p=.04$. The other specific PDs were not related to pretest scores (Table 3 ).

At the posttest and FU 1 and FU 2 test, the presence of one or more full-blown PDS, regardless of type, was not related to change scores ( $p s \geq .31$, Table 3 ). Patients with $\mathrm{PD}(\mathrm{s})$ improved parallel to patients without a PD. Figure 1(1-6) shows the 
mean ratings on the individual outcome measures of patients with $\mathrm{PD}(\mathrm{s})$ and without a PD. Note that statistical analyses were performed using one general composite outcome measure, instead of analyzing the individual outcome measures as presented in Figure 1. When patients with merely subthreshold PD diagnoses were included in the PD-group as well, the PD group still improved parallel with the group of patients without a PD ( $p s \geq .45$, Table 3 ).

As far as specific PDs were concerned, none of them were significantly related to change scores from pretest to later tests ( $p s \geq .11$, Table 3 ). At the Fu 2 test, patients with a paranoid $\mathrm{PD}$ tended to improve more than patients without a paranoid PD ( $p=.06$, Table 3). Patients with a schizotypal PD tended to improve less than patients without a schizotypal PD $(p=.11$, Table 3$)$.

TABLE 3

Effect of categorical personality disorder (PD) variables on pretest scores and treatment outcome of OCD patients

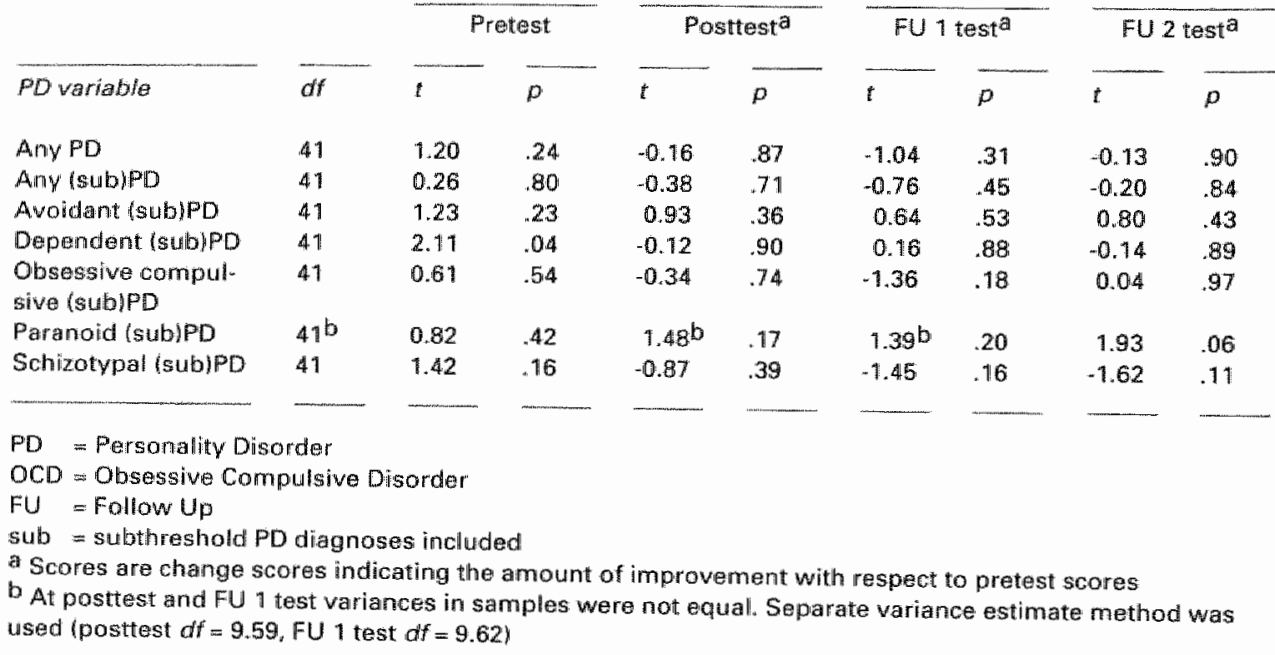


1.1 BAT-1| (expectation by patient, $0-40$ )

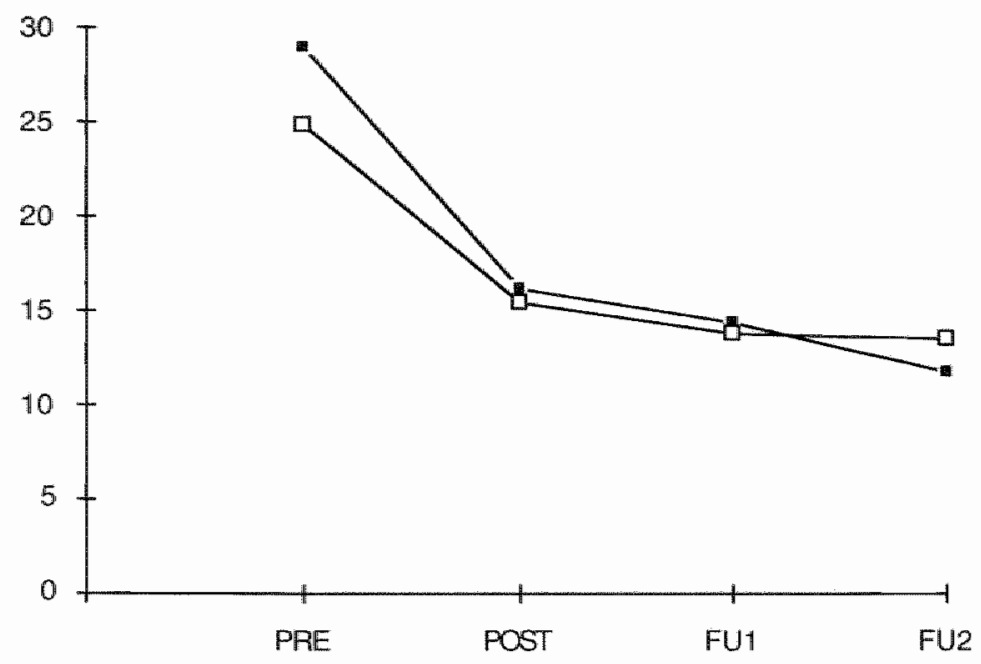

1.2 BAT-2 (clinical observation, $0-40$ )

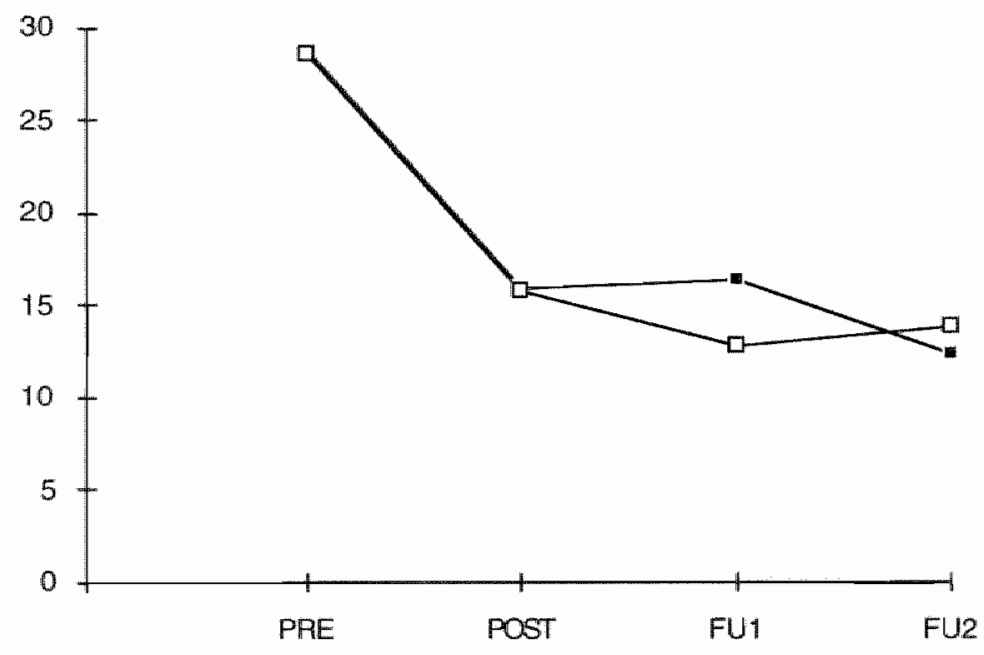

FIGURE 1 (1-6)

Mean ratings of the individual outcome measures of patients with one or more personality disonders ( $)$ and without a personality disorder $(\square)$ at pretest, posttest and follow up tests 
1.3 Maudstey Obsessional Compulsive Inventory (0-30)

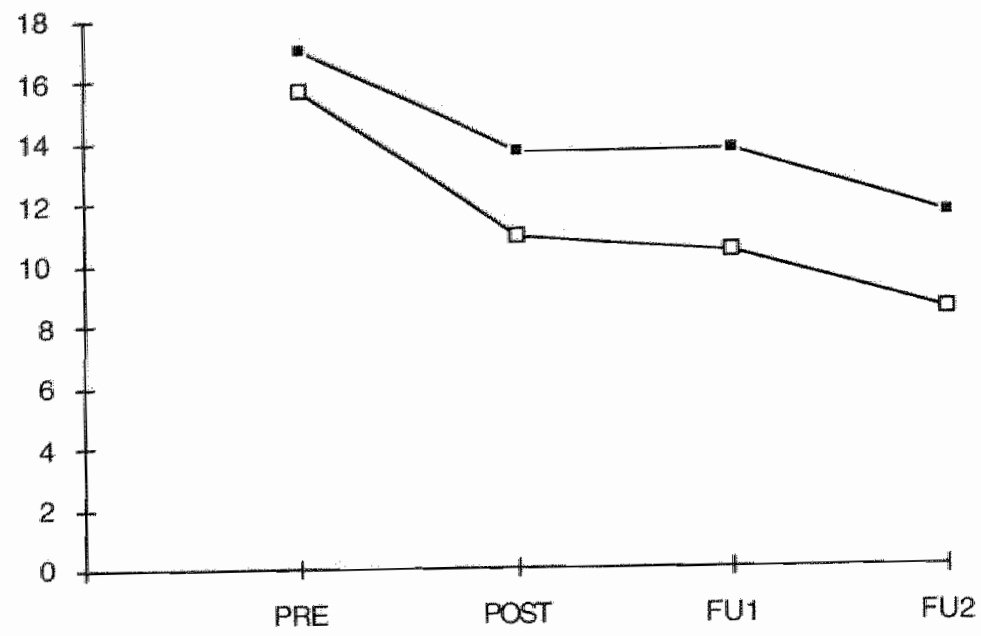

1.4 Leyton Obsessional Invertory $(0-23)$

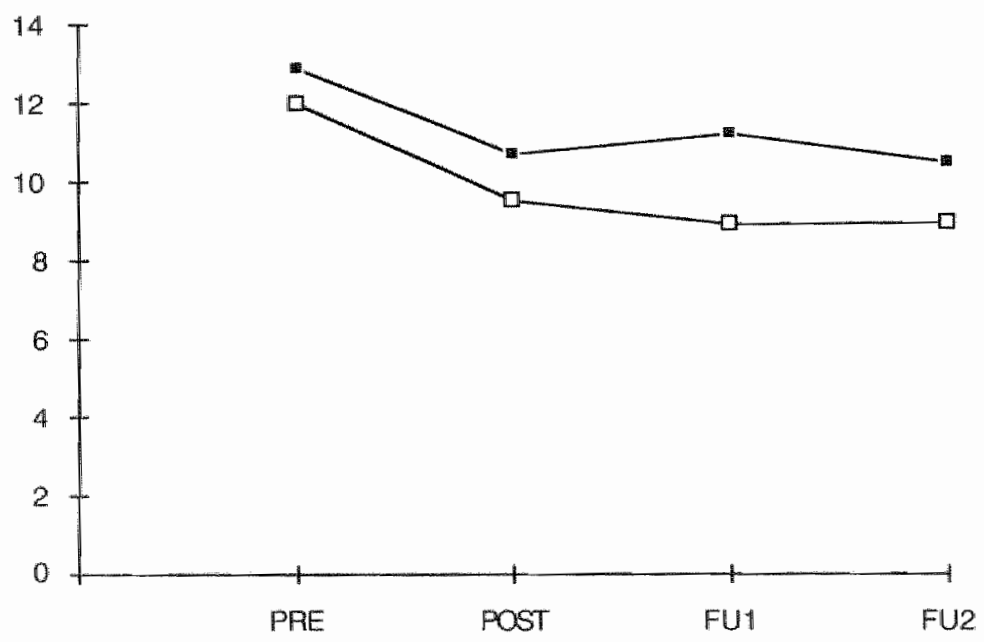

FIGURE 1 (1-6) (continued)

Mean ratings of the individual outcome measures of patients with one or more personality disorders () and without a personality disorder (J) at pretest, postest and follow up tests 


\subsection{Rational Belief Inventory (0-38)}

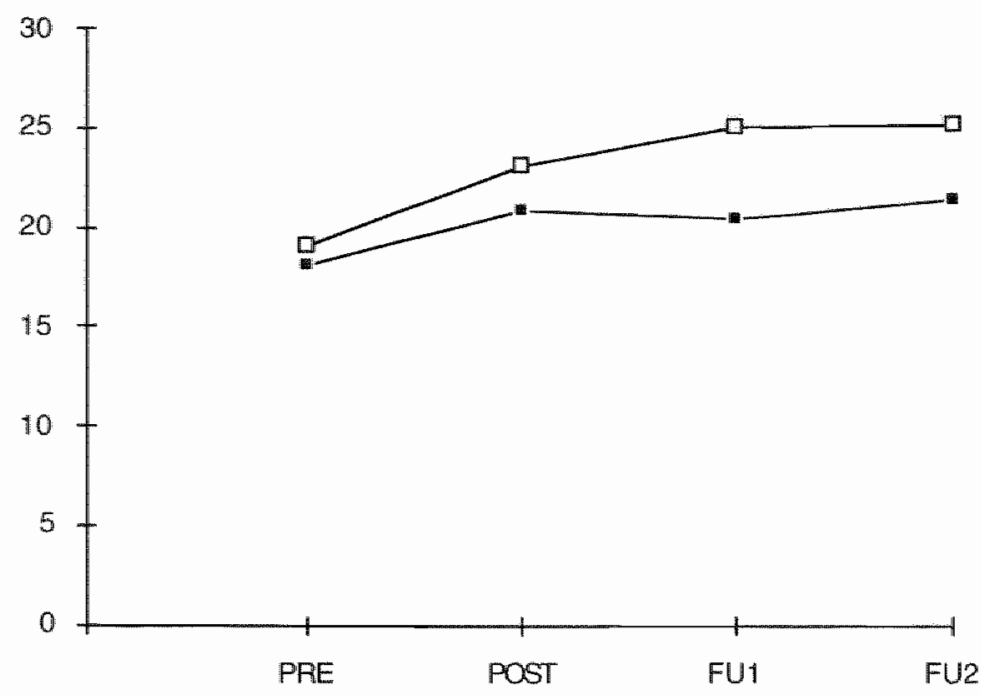

1.6 Depressive Symtoms Inwentory (22-110)

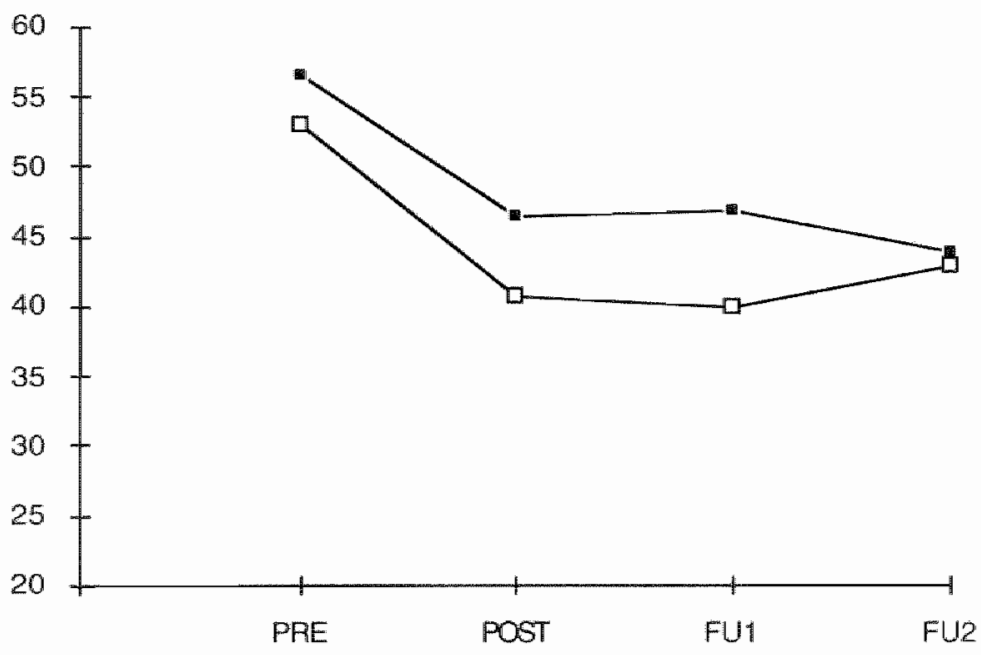

FIGURE 1(1-6) (continued)

Mean ratings of the individual outcome measures of patients with one or more personality disorders ( $)$ and without a personality disorder ( $(\square)$ at pretest, posttest and follow up tests 


\section{Effect of Dimensional Personality Ratings}

The predictive value of the following dimensional personality ratings was examined by means of linear regression analyses:

1 total number of PDS, with or without subthreshold diagnoses;

2 total number of SCID-II personality traits;

3 traitscores of specific PDs;

4 sumscores of specific PDs.

Sumscores of specific pDs were obtained by adding up all scores that were given on the traits concerned $(1,2$ or 3$)$. Sumscores included additional information about personality characteristics that were not scored as full-blown traits. For example, the avoidant traitscore ranged from $0-7$, whereas the avoidant sumscore ranged from 7-21.

The effect of trait- and sumscores were tested by means of stepwise regression analyses. Avoidant, dependent, obsessive compulsive, paranoid, schizotypal, passive aggressive, and self-defeating trait and sumscores were included in the stepwise regression analyses. Other PDs were excluded because of low variance (less than 10 cases being diagnosed with two or more traits of that particular PD).

The results concerning all dimensional personality variables are presented in Table 4. At the pretest, the total number of PDs predicted a higher initial level of symptomatology ( $B=0.32, t=2.13, p=.04)$. The total number of SCID-II personality traits did not do $s o(B=0.25, t=1.62, p=.11)$. Stepwise regression analyses revealed that only schizotypal traitscore $(B=0.33, t=2.22, p=.03)$ and self-defeating sumscore $(B=0.33, t=2.23, p=.03)$ significantly predicted higher pretest scores.

In the analyses of treatment outcome (change with respect to pretest) the pretest composite score was forced into the equation, to control for its contribution to outcome. The pretest composite score was no significant predictor in any of the regression analyses. At posttest FU 1 and FU 2 test, the total number of PDs (with or without subthreshold diagnoses) and the total number of personality traits had no significant predictive value ( $p s \geq .71$, Table 4). With regard to specific PDS, none of the traitscores were a significant predictor of treatment outcome at any of the assessment points ( $p s \geq .13$, Table 4 ). The same held for the sumscores ( $p s \geq .10$, Table 4). Of all trait and sumscores only schizotypal sumscore had a standardized $B$ coefficient below $-0.25(B=-0.27, p=10)$. Negative $B$ coefficients indicate that the personality score was associated negatively with the improvement of complaints. 
TABLE 4

Effect of dimensional personality disorder (PD) variables on pretest scores and treatment outcome. of $O C D$ patients

\begin{tabular}{|c|c|c|c|c|c|c|c|c|c|c|c|}
\hline \multicolumn{3}{|c|}{ Pretest } & \multicolumn{3}{|c|}{ Posttest } & \multicolumn{3}{|c|}{ FU 1 test } & \multicolumn{3}{|c|}{ FU 2 testa } \\
\hline$B$ & it & $\rho$ & $B$ & $t$ & $p$ & $B$ & $t$ & $p$ & $B$ & $t$ & $p$ \\
\hline 0.32 & 2.13 & .04 & 0.06 & 0.38 & .71 & $-0,03$ & $0.2 \pi$ & 84 & 0.04 & 0.07 & .94 \\
\hline 0.30 & 1.95 & .06 & 0.03 & 0.17 & .87 & $-0,03$ & -0.45 & .88 & -0.03 & -0.20 & .85 \\
\hline 0.25 & 1.62 & .11 & 0.05 & 0.32 & .75 & 0.04 & 0.24 & 81 & 0.00 & 0.02 & .99 \\
\hline
\end{tabular}

\section{Stepwise regression}

analyses

Traitscore of specific PDs

\begin{tabular}{|c|c|c|c|c|c|c|c|c|c|c|c|c|}
\hline Avoidant & 0.10 & 0.61 & .54 & 0.02 & 0.12 & .90 & 0.05 & 0.31 & .76 & 0.05 & 0.32 & .75 \\
\hline Depeniclent & 0.11 & 0.74 & .46 & 0.04 & 0.27 & .79 & 0.08 & 0.52 & 61 & .0 .02 & -0.111 & .91 \\
\hline Obsessive compulsive & -0.06 & -0.38 & .71 & 0.02 & 0.13 & .90 & -0.09 & -0.59 & .56 & 0.02 & 0.15 & .88 \\
\hline Passiva aggressive & 0.11 & 0.73 & .47 & -0.21 & -1.33 & .19 & -0.20 & -1.25 & .22 & -0.14 & -0.92 & .36 \\
\hline Selfodafeating & 0,19 & 1.21 & .24 & 0.21 & 1.31 & .20 & 0.25 & 1.53 & 13 & 0.09 & 0.5 .4 & .59 \\
\hline Paranoid & -0.06 & -0.38 & .70 & 0.22 & 1.39 & .17 & 0.19 & 1.20 & .24 & 0.20 & 1.32 & .19 \\
\hline Schizotypal & 0.33 & 2.22 & .03 & -0.01 & -0.07 & .94 & -0.10 & -0.59 & .56 & -0.118 & -1.13 & .27 \\
\hline \multicolumn{13}{|l|}{ Sumscore of specific PDs } \\
\hline Avoidant & 0.07 & 0.45 & .66 & -0.00 & -0.03 & .98 & 0.03 & 0.18 & .86 & 0.04 & 0.26 & .80 \\
\hline Dependent & 0,04 & 0.21 & .84 & 0.06 & 0.38 & .71 & 0.12 & 0.74 & 46 & 0.02 & 0.13 & .90 \\
\hline Obsessive compulsive & -0.05 & -0.35 & .73 & 0.03 & 0.18 & .86 & .0 .07 & -0.44 & 66 & 0.01 & 0.04 & .97 \\
\hline Passive aggressive & 0.06 & 0.29 & .77 & -0.17 & -1.10 & .28 & -0.14 & $-0,86$ & .40 & -0.10 & -0.66 & .52 \\
\hline Self-defeating & 0.33 & 2.23 & .03 & 0.21 & 1,31 & .20 & 0.26 & 162 & 11 & 0.07 & 0.42 & .68 \\
\hline Palranoid & -0.05 & -0.35 & .73 & 0.23 & 1.46 & .15 & 0.21 & 1.33 & 19 & 0.22 & 1.41 & .17 \\
\hline Schizotypal & 0.19 & 1. 18 & .24 & -0.04 & -0.25 & .80 & -0.12 & -0.73 & .47 & -0.27 & $-1,69$ & .10 \\
\hline
\end{tabular}

In the regrassion analyses on postest and FU test scores the composite pretest score was forced in the equation. The f coefficient represents the standardized regression coefficient. A negative is coefficient indicates that the PD variable was associated negatively with improwement of complaints. The alpha to enter the regression equation was set at .05

$\mathrm{PD}=$ Personality Disolder

$\mathrm{OCD}=$ Obsessive Compulsive Disorder

$\mathrm{FU}=$ Follow Up

a Scores are change scores indicating the annount of improvement with respect to pretest scores

\section{Results when drop-outs were included in the analyses (general composite score)}

Data of the nine drop-outs were included in the analyses. Since drop-outs only had pretest scores, change scores had to be estimated. Based on the judgment of the therapist, two categories of drop-outs were distinguished. (a) Drop-outs without any improvement regarding their OCD complaints $(n=8)$. Change scores were set at zero. (b) Drop-outs with considerable improvement regarding their OCD complaints $(n=1)$. Change scores with respect to pretest scores were obtained by subtracting the mean amount of improvement of patients in that particular treatment condition from the individual pretest score of the patient who dropped out. Using this procedure, the possible therapist biases cannot be precluded, but a better alternative was not available. 


\section{Effect of Categorical PDs, Drop-Outs Included}

All $t$-tests with categorical variables were repeated and the results were similar to results obtained with the patients that completed the treatment protocol. At the pretest, the dependent PD was still the only diagnosis that was significantly related to the general composite pretest score, $t(48)=2.16, p=.04$. Patients with dependent Po had a higher level of initial complaints than patients without a dependent $\mathrm{PD}$. None of the categorical variables were significantly related to change scores from pretest to later tests ( $|t s| \leq 1.79, p s \geq .08$ ).

Before including the drop-outs, patients with a paranoid $\mathrm{PD}$ tended to improve more than patients without a paranoid PD at the FU 2 test ( $p=.06$, Table 3 ). This trend disappeared when drop-outs were included in the analyses, $t(50)=1.49, p=$ .14. The trend for patients with a schizotypal PD to improve less than patients without a schizotypal $P D(p=11$, Table 3 ) slightly increased by including the dropouts in the analyses, but failed to reach the statistical level of significance, $t(50)=$ $-1.79, p=.08$.

\section{Effect of Dimensional Personality Ratings, Drop-Outs Included}

When drop-outs were included, the predictive value of the total number of PDS for initial levell of symptomatology decreased slightly $(i s=0.27, t=1.91, p=.06)$. Stepwise regression analysis revealed that schizotypal traitscore and self-defeating sumscore no longer significantly predicted higher pretest scores $(B=0.11, t=0.55$, $p=.58 ; B=0.19, t=1.00, p=.32$ ).

With regard to change scores at posttest, FU 1 and FU 2 test, none of the dimensional personality ratings were predictors of significance ( $\mid B s \| \leq 0.22$, $\mid$ ts $\mid$ $\leq 1.51, p s \geq .14)$. These findings were completely in line with the results found when drop-outs were excluded from the analyses. The predictive value of schizotypal sumscore at the FU 2 test found in the group of patients that completed the treatment protocol $(p=.10$, Table 4$)$, did not increase by including drop-outs in the analyses $(B=-0.21, t=1.50, p=.14)$.

\section{Effects of PD variables on a composite measure of MOCI and BAT-1 and BAT-2 scores}

With regard to the categorical PD variables (any PD with or without subthreshold diagnoses, avoidant, dependent, obsessive compulsive, paranoid and schizotypal pD) none significantly affected treatment outcome with respect to the composite score of the MOCr and the BATs (change with respect to pretest, $\mid$ ts $\mid \leq 1.61, p s \geq$ .11). These results did not change when the drop-outs were included in the analyses ( $|\mathrm{fs}| \leq 1.73$, $p s \geq 09$ ), but there was a trend toward significance for the schizotypal PD to affect the change score negatively at the Fu 1 test, $t(50)=-1.73, p$ $=.09$. At the fu 2 test this trend disappeared, $t(50)=-1.48, p=.15$.

With regard to the dimensional PD variables (number of PD diagnoses with or without subthreshold diagnoses, number of SCID-II traits and the trait- and sumscores of individual PDS) none were found to be statistically significantly related to treatment outcome of OCD symptoms (change with respect to pretest, $\mid$ Bs $\mid \leq$ $0.31,|t s| \leq 2.02, p s \geq .05)$. However, there was a trend toward significance for the passive aggressive trait- and sumscore to relate negatively to the posttest change 
scores $(B=-31, t=-2.02, p=.05$, resp. $B=-.28, t=-1.84, p=07)$. At the fu tests these trends disappeared ( $|B s| \leq 0.22,|t s| \leq 1.37, p s \geq 18$ ). Similarly, thene were no statistically significant relationships between dimensional po variables and change scores at posttest or Fu tests after inclusion of the drop-outs (|Bs $|\leq 0.23| ,1 \mathrm{~s} \mid \leq$ $1.60, p s \geq .12)$.

\section{Clinical significance analyses}

Patients were considered improved if the RC index was $\geq 1.96$. For the RC indexes to be $\geq 1.96$, the change score on the MocI had to be $\geq 11$, on the BAT-1 it had to be $\geq 19$, and on the BAT-2 it had to be $\geq 23$. In order to be classified as recovered, posttest or FU test scores on the MOCI had to be $\leq 7$, on the BAT-1 they had to be $\leq 10$ and on the BAT-2 they had to be $\leq 8$. Note that patients had to improve a lot to be considered improved or recovered, because the standard deviations in the sample were relatively high. To avoid the problem of different cutoff points from study to study, Jacobson and Truax (1991) prefer to use norms of the clinical and nonclinical populations. However, these were not available for the MOCI and the BATs.

The percentages of patients who improved and recovered according to the criteria of Jacobson and Truax (1991) are shown in Table 5. No statistically significant differences were found on any of the outcome measures at any of the assessment points between the number of patients with and without PDs that improved or recovered (chi-square or Fisher's Exact tests, $p s \geq .07$ ). These results did not change when the drop-outs were included (ps $\geq .09$ ).

TABLE 5

Percentages of completers with no personality disorder (PD) $(n=21)$ or with one or more PDs ( $n$ $=22$ ) that improved and recovered on the OCD outcome measures

\begin{tabular}{|c|c|c|c|c|c|c|c|c|c|c|c|c|}
\hline & \multirow{2}{*}{\multicolumn{4}{|c|}{ Postlesta }} & \multirow{2}{*}{\multicolumn{4}{|c|}{ Fu 1 test }} & \multirow{2}{*}{\multicolumn{4}{|c|}{ FU 2 test }} \\
\hline & & & & & & & & & & & & \\
\hline & \multicolumn{2}{|c|}{ Improved } & \multicolumn{2}{|c|}{ Recovered } & \multicolumn{2}{|c|}{ Improved } & \multicolumn{2}{|c|}{ Recowered } & \multicolumn{2}{|c|}{ Improwed } & \multicolumn{2}{|c|}{ Recovered } \\
\hline OCO measures & WO $P D$ & $\begin{array}{l}\text { One or } \\
\text { more } \\
\text { PDs }\end{array}$ & NOPD & $\begin{array}{l}\text { One or } \\
\text { more } \\
\text { PDs }\end{array}$ & NOPO & $\begin{array}{l}\text { One or } \\
\text { moro } \\
\text { PDs }\end{array}$ & No $P D$ & $\begin{array}{l}\text { Ons or } \\
\text { mane } \\
\text { PDs }\end{array}$ & NopD & $\begin{array}{l}\text { One ar } \\
\text { mores } \\
P D s\end{array}$ & NoFD & $\begin{array}{l}\text { One or } \\
\text { more } \\
\text { PDs }\end{array}$ \\
\hline MOCl & 5 & 1.4 & 33 & 23 & 19 & 14 & 33 & 32 & 38 & 23 & $52^{a}$ & $27^{a}$ \\
\hline $\begin{array}{l}\text { BAT- } \\
\text { lexpectation } \\
\text { by patient) }\end{array}$ & 14 & 1.4 & 33 & 27 & 19 & 27 & 43 & 46 & 19 & 32 & 48 & 54 \\
\hline $\begin{array}{l}\text { EAT-2 } \\
\text { (clinical } \\
\text { abservation) }\end{array}$ & 19 & 18 & 33 & 36 & 23 & 18 & 43 & 36 & $10^{b}$ & $36^{b}$ & 43 & 41 \\
\hline
\end{tabular}

OCD = Obsesivive Compulsive Dusorder

$\mathrm{FU}=$ Follow $\bigcup_{\mathrm{p}}$

a Two-Tailed Fisher"s Exact tesit: $p=07$

b Two-Tailled Fisher's Exact test: $p=.09$ 


\section{Discussion}

To the best of our knowledge, the present study is the first one that has used a (semi)structured interview for the assessment of DSM-III-R PDS in studying the influence of $\mathrm{PDS}$ on $\mathrm{OCD}$ treatment outcome. Two composite measures were evaluated: a general composite measure including all measurements taken and a specific composite measure containing measures of $O C D$ symptoms targeted in the treatment. Results show that OCD patients with and without PDs benefit equally from $C B T$ focused on Axis 1 OCD complaints. In addition, when different types of PDs are studied separately, there is no evidence from the present study for a negative impact on OCD treatment outcome.

Besides categorical personality variables, we also studied the effect of dimensional personality variables. The dimensional approach is favored above the categorical approach by several authors (see Widiger, 1992, p. 288). Across studies, dimensional personality ratings are found to be more reliable and valid than categorical diagnoses, the thresholds of which are largely arbitrary (Widiger, 1992). It can be postulated that personality traits influence treatment outcome before the minimum set of criteria for a PD is reached, or that many more criteria are needed than just the minimum set of criteria required for a DSM-MII-R PD diagnoses. In the present study no evidence was found for this reasoning: results on dimensional variables confirmed the findings on categorical personality variables, with a few exceptions concerning the effect on pretest scores. For instance, patients with one or more PDS (categorical variable), did not have a higher level of complaints at pretest than patients without a $\mathrm{PD}$, but the total number of $\mathrm{PD}$ diagnoses that a patient had (dimensional variable), correlated positively with the initial level of complaints at pretest.

Finally, we classified patients as being improzed or unimproved and as being recovered or unrecovered with respect to the OCD complaints. The presence of one or more PDS did not affect the percentage of improved or recovered OCD patients.

A matter of great importance when studying the effect of personality variables on treatment prognosis is the question of whether patients with concomitant $\mathrm{PD}$ tend to select themselves out of standardized treatment protocols. If that is the case, it would be too optimistic to conclude from analyses with patients who completed the protocol that personality pathology does not affect treatment outcome of $O C D$. If patients fail to attend treatment sessions, they cannot benefit from therapy. With regard to all categorical and dimensional personality variables, the results did not change when drop-outs were included in the analyses. In conclusion, the present study shows that personality pathology measured by means of the SCID-11 interview does not affect treatment outcome of OCD patients in general, treatment adherence included. Results of the present study cannot be generalized to schizoid, histrionic, narcissistic, borderline and antisocial personality traits because they were diagnosed infrequently in our study sample.

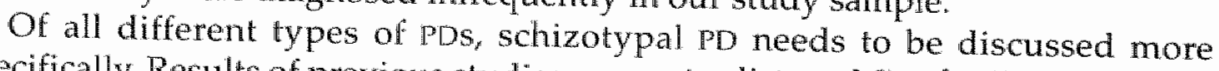
specifically. Results of previous studies are contradictory. Minichiello et al. (1987) found a negative effect of this PD on OCD treatment outcome and Fals-Stewart and Lucente (1993) found a negative effect of a particular cluster of personality features that included schizotypal features. In line with the findings of the Steketee (1990) 
study the present study does not provide convincing evidence for a negative impact of this $\mathrm{PD}$, but there is a tendency for patients with schizotypal features to improve less than other patients. This trend did not increase by including dropouts in the analyses. A plus point of our study, compared to previous studies, is that we used a semi-structured instrument in a prospective design. A minus point of our study is that our sample size was relatively small, compared to the sample size of 137 patients in the Fals-Stewart and Lucente (1993) study. Further research using large sample sizes and (semi)structured personality interviews are needed to confirm our conclusion that schizotypal PD does not affect $\mathrm{OCD}$ treatment outcome negatively.

The present findings go against the clinical lore. Clinicians believe that PDs affect treatment outcome negatively. Several explanations for this discrepancy between empirical research and clinical impression can be considered.

First, therapists who evaluate the personality of the patient during or at the end of treatment are not blind to treatment outcome. Consequently, therapy failure might increase false positive PD diagnoses. PDs should be defined by criteria other than lack of therapeutic progress.

Second, if therapists have negative beliefs about the outcome of treatment in advance, because the patient has been diagnosed with a $\mathrm{PD}$, it might become true at the end of therapy as a self-fulfilling prophecy.

Third, personality variables that affect treatment may be very specific. Just one DSM-III criterion, such as a low tolerance for strong emotions or just one deeply ingrained belief that effort to change is useless, can be sufficient to cause significant impairment in the treatment process. Analyzing PDs or the number of traits does not take into account such specific personality variables.

Fourth, therapists may be right when they claim that PDs complicate the treatment process. However, it may be that (cognitive-behavioral) therapists nowadays are more eager or able to prevent negative treatment outcome with respect to Axis I disorders by adapting successfully to the interpersonal style of the patient, or by specifically addressing dysfunctional attitudes or schemas in a successful way. We are presently attempting to study this question systematically. ${ }^{3}$

Another point that needs to be discussed is the fact that $56 \%$ of the patients needed additional treatment for their OCD complaints after completion of the 12session protocol. There was no statistically significant difference between patients with and without PDS regarding the number that received additional treatment. Therefore, it seems unlikely that the parallel improvement of OCD patients with PDs compared to patients without any PD was dependent on the additional treatment given between the FU 1 and FU 2 tests. However, we do not know how the progress of OCD complaints evolves after the FU 2 test. Possibly, patients with and without PDs improve parallel up to a certain point (at least Fu 2), after which patients with PDs improve less than patients without a PD. In that case, the presumption that PDS have a negative impact on treatment outcome with OCD might prove to be right after all. Future studies should include longer follow-up periods to test this hypothesis. Moreover, the total number of weekly sessions in the standardized treatment protocol should be increased and a minimum number of sessions to be completed by the patient in order to be considered a study completer should be determined. 
Finally, it can be questioned whether lack of power is a main reason for the non-significant findings. Generally, lack of power may occur when sample sizes are small and when base rates of PDS are low. In the present study, the prevalence rate of OCD patients with one or more full-blown PDs was rather high ( $51 \%$ in the group of completers) and there were not even trends in the hypothesized direction for the general $\mathrm{PD}$ variables such as presence of any $\mathrm{PD}$, number of PD diagnoses or number of personality traits. Therefore, larger sample sizes are unlikely to reveal a negative impact regarding these PD variables. With regard to specific PDs, we required at least 6 positive cases (subthreshold diagnoses included) for the disorder to be included in the analyses. It could be argued that a higher rate of cases that meet the full criteria for that PD might produce different results. However, of all specific PD variables only the schizotypal PD showed a trend toward a negative influence on treatment outcome. What our study certainly shows is that there is no robust negative impact of specific PD variables. Future studies incorporating a larger sample size and higher prevalence rates of specific full-blown PDs (notably schizotypal) might reveal more subtle influences of $P D$ variables on $C B T$ results with $O C D$.

To conclude, results of the present study imply that patients with a DSM-III-R PD should not be excluded from CBT" for OCD complaints in advance. Compared to patients without PDS, they benefit equally from CBT at least until six months after the standardized therapy has finished. Furthermore, therapists should be careful in attributing OCD therapy failure to DSM-III-R PDS, since it appears that PDS assessed in a prospective way by means of a semi-structured interview, do not affect treatment outcome of OCD.

\section{notes}

1 Published as: Dreessen, $L_{\text {. }}$ Hoekstra, $\mathbb{R}$, \& Arntz, A. (1997). Personality disonders do not influence the results of cognitive and behavior therapy for obsessive compulsive disorder. Jownm of Anxiety Disorders, 11, 503-521.

2 When section 3.2 was written, data on the short-interval test-retest interrater reliability of the SCID II in our group of raters, were not amalyzed yet (see section 2.1 of the present thesis). Overall, these data showed satisfactory short-interval test retest interalker reliability of the SCID-II in oul group of raters and patients.

3 Rosults of this study are presented in section 3.3 . 


\title{
Personality disorders have no excessively negative impact on the therapy process in the cognitive and behavioral treatment of Axis I anxiety disorders 1
}

\begin{abstract}
Summary.
The impact of DSMIM-R personality pathology on the Axis I therapy process was studied in 70 outpatients who received cognitive and/or behavioral therapy for an Axis I anxiety disonder. The Axis I therapy process was evaluated by the therapist. DSM II-R personality pathology was assessed first by an independent rater using a semistructured interview (Structured Clinical Interview for DSM-II-R Personality Disorders [SCD-M]) and second by the therapist in the course of treatment Results showed poor diagnostic agreement between scio-and therapist-derived personality pathology. SCDderived personality pathology was only weakly melated to a dysfunctional Axis I therapy process, as reported by the therapist. Therapist-derived personality pathology, on the contrary, was strongly related to a dysfunctional Axis I therapy process, as reported by the therapist. Possibly, therapists erroneously attributed Axis I therapy failure to assumed personality pathology. This is the first study on the impact of personality pathology on the Axis I therapy process. The findlings are consistent with studies that show no negative impact of personality pathology, as assessed by independent semistructured interviews, on therapy ontcome of Axis I anxiety disorders.
\end{abstract}

\section{Introduction}

The impact of personality disorders on therapy outcome of Axis 1 disorders (American Psychiatric Association, 1987) has been investigated in numerous studies (see reviews: Dreessen \& Arntz, 1998-a; Reich \& Vasile, 1993). However, no study could be traced investigating the impact of personality disorders on the Axis I therapy process.

Various problems in the process of therapy for Axis I disorders caused by personality disorders have been postulated (e.g. Beck, Freeman, \& Associates, 1990; Benjamin, 1993; Horowitz, Rosenberg, \& Bartholomew, 1993; Padesky, 1988; Van Velzen \& Emmelkamp, 1996). For example, particular cognitions and beliefs have been hypothesized to interfere with therapy directly (e. g. "There's no use trying, because it won't work anyway' or 'It's better to do nothing than to perform badly'). Other hypothesized problems are low tolerance for strong emotions (e.g. anxiety and grief), interpersonal problems with the therapist (e.g. distrust, Cear of rejection), 
and rigidity of Axis I symptoms because they are interconnected with personality psychopathology. Consequently, the compliance with therapeutic guidelines and homework assignments is believed to be poor, the motivation for change is believed to be low, and the therapeutic relationship is believed to be complicated in patients with personality disorders.

The quality of the therapeutic relationship has been found to correlate positively with outcome in Axis I therapies (Garfield, 1994; Rau \& Goldfried, 1994). However, no study has ever investigated the correlation between personality pathology and the quality of the therapeutic relationship. Similarly, "difficult' patients have been found to affect therapist competence negatively in the treatment of major depressive disorder (Foley, OMalley, Rounsaville, Prusoff \& Weisman, 1987). However, no study has investigated whether the presence of personality disorders predicts whether patients are more 'difficult' in the treatment of Axis I disorders.

On the face of it, complications in the Axis I therapy process caused by personality disorders seem very plausible and one would expect the Axis in therapy outcome to be poorer in cases with concomitant personality disorders than in those without. However, with respect to anxiety disorders, there is now increasing evidence that patients with and without personality disorders show an equal amount of improvement in their Axis I anxiety disorder symptoms (e.g. Dreessen, Arntz, Luttels, \& Sallaerts, 1994; Dreessen, Hoekstra, \& Arntz, 1997; Mellman et al, 1992; Mersch, Jansen, \& Amtz, 1995; Noyes et al., 1990; Sanderson, Beck, \& McGinn, 1994; Van Velzen, Emmelkamp, \& Scholing, 1997). An interesting question is how this parallel improvement was brought about. Possibly, personality disorders did not complicate the Axis I therapy process to a great extent. Or, personality disorders did indeed complicate the Axis I therapy process, but the therapists handled the complication successfully (e.g. by adapting successfully to the interpersonal style of the patient or by changing therapy interfering cognitions in a successful way) and achieved an equal amount of improvement in the Axis I complaints. If personality disorders did not complicate the Axis I therapy process, the question is whether therapists perceived any personality pathology at all while they were treating the patient for the Axis $₫$ disorder.

The purpose of the present study was to investigate the impact of DSM-III-R personality pathology on the therapy process in treating Axis I anxiety disorders. In addition, it was investigated whether therapists in an Axis therapy for anxiety disorders perceive any DSM-III-R personality pathology as diagnosed with an independent semistructured interview. The Axis I therapy process was evaluated by the therapists by completing a self-constructed questionnaire. Personality pathology was assessed by an independent rater using the Structured Clinical Interview for DSM-HI-R personality disorders (SCID-II; Spitzer, Williams, Gibbon, \& First, 1990). To measure personality pathology as perceived by the therapist, therapists completed a self-constructed questionnaire evaluating personality pathology of the patient whom they were treating for an Axis I anxiety disorder. The following research questions were tested:

1 Do therapists perceive any SCID-personality pathology in the treatment of Axis I anxiety disorders? In other wonds, how strong is the diagnostic agreement between therapist-perceived personality pathology and SCID-personality pathology? 
2 Does SCID-personality pathology relate to complications in the Axis I therapy process?

3 Does therapist-perceived personality pathology relate to complications in the Axis I therapy process?

4 Do therapists experience being able to surmount the complications in the Axis I therapy process caused by personality pathology (as measured by the SCID-II and as perceived by the therapist)?

\section{Method}

\section{Patients}

The participants were 70 outpatients who were referred to the "Academic Section Behavior Therapy' of the Community Mental Health Center at Maastricht. Following everyday practice in the academic section, an intaker administered an unstructured clinical interview, followed by the Structured Clinical Interviews for DSM-III-R Axis I and Axis II disorders (SCID: Spitzer, Williams \& Gibbon, 1987; Dutch translation: Koster van Groos, 1985; revision by Arntz, Bögels, \& Hoekstra, 1992-a; SCDD-II: Spitzer et al., 1990; Dutch translation: PC Bloemendaal, 1991). The main Axis I diagnosis in all patients was an anxiety disorder (29 patients with a panic disorder with/without agoraphobia, 22 with a social phobia, 6 with a generalized anxiety disorder, 6 with an obsessive compulsive disorder, 5 with a posttraumatic stress disorder, 1 with an agoraphobia, and 1 with a simple phobia). Furthermore, according to the SCID for Axis I disorders, 20 patients had one or more concomitant anxiety disorders, 15 had a concomitant depressive disorder, 3 had a concomitant somatoform disorder and 1 patient had a concomitant eating disorder. According to the unstructured clinical interview, 2 patients abused alcohol, 1 had concomitant sexual dysfunctions, and 6 patients had a V-Code problem ( 1 bereavement and 5 relational problems). The prevalences of SCID-Axis II diagnoses are mentioned in the Results section. 29 men and 41 women participated in the study. The mean age was 35 years (range 19-60), $74 \%$ were married or living together with a partner.

\section{SCID-II Interviewers}

Ten different SCID-II interviewers participated in the study. All of them were experienced in diagnosing and treating psychotherapy patients and practiced as therapists in the 'Academic Section Behavior Therapy'. Five of them (A.A., L.D., M.vR., W.K., H.vG.) had participated in our SCID-Il reliability studies which demonstrated good interrater reliabilities (Arntz et al., 1992-b; Dreessen \& Arntz, 1998-b). The other five interviewers were trained extensively by doing joint interviews with several experienced interviewers who participated in our reliability studies. At first, the trainees were second rater. Later on, they were first rater. After each joint interview, trainer and trainee discussed the elicitation and interpretation of information.

\section{Therapy}

All patients were treated for an Axis I anxiety disorder. They received cognitive or behavioral therapy or a combination of both. Most of the therapies were 
standardized, meaning that therapists followed treatment manuals within the framework of comparative therapy-effect studies. If patients did not participate in a therapy-effect study $(n=6)$, therapists were free to chose any kind of cognitive and/or behavioral therapy. All patients followed an individual treatment, except for 15 patients with panic disorder, who followed a standardized group treatment.

\section{Therapists}

During the investigation period, 14 therapists treated at least one patient from the study. All therapists were well trained in cognitive and/or behavioral treatment techniques (e.g. by D.M. Clark, P. Salkovskis, C. Padesky, L. G. Ost, C. Fairburn, J. Young, A. T. Beck, \& A. Arntz). They were officially registered behavior therapists or in training toward becoming such a therapist.

\section{Instruments}

Structured Clinical Interview for DSM-III-R Personality Disoräers. The (semi)Structured Clinical Interview for DSM-III-R Personality Disorders (SCID-II; Spitzer et al., 1990; for a full description see First, Spitzer, Gibbon, \& Williams, 1995-a) was used for the assessment of personality pathology (Dutch translation: PC Bloemendaal, 1991). The SCID-IT systematically addresses all DSM-III-R personality traits. In our academic section, it is common practice to assess all SCID-II traits without using the SCID-Personality questionnaire as a first screening (First et al., 1995-a). At the end of the SCID-II interview the interviewer rated the overall quality of the information elicited in terms of 'poor', 'fair', 'good', or 'excellent'.

Psychometric properties of the SCID-II have been found to be satisfactory (e.g. Arntz et al., 1992-b; Dreessen \& Arntz, 1998-b; First et al., 1995-b; O'Boyle \& Self, 1990; Renneberg, Chambless, Dowdall, Fauerbach, \& Gracely, 1992; Skodol, Oldham, Rosnick, Kellman, \& Hyler, 1991).

Therapist Questionnaire for Personality Pathology. In order to obtain therapist evaluations of the personality pathology of the patient, we constructed a short questionnaire. Instructions to the questionnaire contained the general DSM-III-R criteria for any personality disorder and also a short characterization of each individual DSM-III-R personality disorder (APA, 1987). Furthermore, the therapist was instructed to give his or her own clinical judgment, regardless of the jud gment of other clinicians, and without interviewing the patient about personality pathology. The questionnaire itself required a dimensional rating for general personality pathology on a $100 \mathrm{~mm}$ visual analogue scale (VAS). The anchors were formulated as 'no personality pathology' and 'extreme personality pathology'. In addition, the questionnaire required 3-point ratings for every individual personality disorder category included in the SCID-II $(0=$ no traits, no disorder; $1=$ traits, 2 = disorder). Therapists were not interviewed by means of the SCID-II, because the separate items of the SCID-II require very detailed knowledge of the patient. Therapists in an Axis I therapy were not expected to have such detailed information about the persionality of the patient.

Therapist Questionnatre for Axis I Therapy Process. The Axis I therapy process was evaluated by the therapist using a self-constructed questionnaire. The questionnaire required dimensional ratings on a $100 \mathrm{~mm}$ VAS for 12 questions addressing the Axis I therapy process (e.g. 'Is the therapy progressing with 
difficulty?' or 'Is the patient active and self-acting in the therapy?'). All individual questions are presented in Table 2 (see Results). Notice that the items $1,3,4$ and 6 address the therapy process in general, whereas in items 2 and 5 , the therapy process is explicitly linked to the personality of the patient.

\section{Procedure}

Following the unstructured diagnostic clinical interview and the SCID for Axis I disorders, written informed consent was obtained from the patients for using the SCID-II data for empirical research. Then, the SCID-II interview was administered. In most cases, the SCID-II interviewer had also done the intake with the patient concerned. If this was not the case $(n=25)$, the SCID-II interwiewer studied the patient file in order to have relevant background information in administering the interview questions. Both the patient and the therapist in attendance were kept blind to the SCID-II diagnoses. Some of the therapists also conducted SCID-II interviews, but not of the patients they treated.

Between the sixth and seventh therapy session (counted from the session in which the therapy rationale was explained) therapists completed the questionnaire on the Axis I therapy process. We reasoned that around the sixth session, therapists would be able to comment on the therapy process and the personality pathology of the patient, without being influenced by the final results of the therapy. In order to maximize independence in answers as much as possible, the questionnaire for evaluating personality pathology of the patient was completed a few days after completing the questionnaire on the Axis I therapy process.

TABLE 1

Prevalence of personality pathology diagnoses according to therapist judgment and SCID-II $(n=70)$

\begin{tabular}{l} 
Personality disorder \\
Avoidant \\
Dependent \\
Obsessive compulsive \\
Passive aggressive \\
Self-defeating \\
Patanoid \\
Schizotypal \\
Schizoid \\
Histrionic \\
Narcissistic \\
Borderline \\
Antisocial \\
Sadistic \\
One or more \\
\hline
\end{tabular}

\section{Personality disorder}

SCID-ll Therapist

\begin{tabular}{rrc}
13 & 11 & 0.10 \\
8 & 4 & 0.10 \\
5 & 5 & 0.14 \\
0 & 1 & $\cdots$ \\
0 & 0 & - \\
4 & 0 &. \\
0 & 0 & - \\
0 & 0 & - \\
1 & 0 & - \\
0 & 0 & - \\
1 & 0 & - \\
0 & 0 & - \\
0 & 0 & - \\
20 & 18 & - \\
\hline
\end{tabular}

Personality trats or disorder

\begin{tabular}{|c|c|c|}
\hline $\begin{array}{l}\text { SCID-ll } \\
\text { te } 2 \text { traitsi }\end{array}$ & Therapist & Kappa: \\
\hline 36 & 81 & 0.10 \\
\hline 38 & 30 & 0.44 \\
\hline 29 & 24 & 0.12 \\
\hline 17 & 17 & -0.01 \\
\hline 5 & 3 & -0.06 \\
\hline 21 & $b$ & 0.30 \\
\hline 28 & 0 & - \\
\hline 6 & 0 & $\cdots$ \\
\hline 4 & 5 & 0.41 \\
\hline 4 & 2 & - \\
\hline 33 & 3 & 0.33 \\
\hline 3 & 0 & - \\
\hline $\mathfrak{0}$ & 0 & " \\
\hline 56 & 61 & 0.12 \\
\hline
\end{tabular}

a Kappa was computed only if at least five cases were diagnosed positivaly by at least one of the wo sources \{lllness base rate requirement: see Dreessen \& Amtz, 1998-bh. Kappa cannot be computed if ullness base rate of one of the two sources is zero 


\section{Resullts}

Diagnostic Agreenent between SCDD-Il and Therapist-perceived Personality Pahology The prevalences of categorical personality pathology diagnoses according to the therapist and according to the SCID-II are presented in Table 1. Diagnostic agreement was estimated only if at least five cases were diagnosed positively by the SCID-II or by the therapist. Agreement was estimated on the presence of a 'disorder" and on the presence of 'traits or disorder". SCID-II 'traits' were rated present if the patient had 2 or more traits of the category concerned. Overall, diagnostic agreement on these categorical personality pathology variables was poor [Cohen's kappas (Cohen, 1960) were smaller than 0.45 , see Table 1].

The dimensional therapist-VAS score for general personality pathology $(M=$ $49, S D=28$ ) correlated only moderately with the total number of SCID-II traits ( $M$ $=10, S D=7)(r=0.39, \mathrm{p}<0.01)$. However, patients with one or more SCID-II personality disorders did show higher therapist-VAS scores $(M=62, S D=28)$ than patients without any SCID-II personality disorder $(M=44, S D=27)[t(68)=$ $-2.45, p=.02]$.

\section{The Impact of Personality Pathology on the Axis I Therapy Process: General Personality Pathology}

As can be seen in Table 2, correlations between SCID-personality pathology (number of traits) and therapy process variables were low ( $\left|r_{s}\right| \leq 0.27$ ), whereas correlations between therapist-perceived personality pathology (VAS score) and therapy process variables were high $(0.53 \leq|r s| \leq 0.70)$ (mean, standard deviation and range of the therapy process variables are shown in Table 2). More specifically, SCIDpersonality pathology was only weakly related to more complications in the therapy process (items 2 and 6: $01<p \mathrm{~s} \leq .05$, Table 2). The effort by the therapist, the efficacy of the therapist effort and the ability of the therapist to stop personality interference did not correlate with SCID-personality pathology (items 3,4 and 5: ps $\geq .05$, Table 2). Interviewers rated the overall quality of each SCID-I interview as poor, fair, good, or excellent. Exclusion of SCID-II interviews with poor or only fair quality ratings did not result in higher correlations between SCD-personality pathology and therapy process complications ( $|r s| \leq 0.26, n=49$ ).

Therapist-perceived personality pathology, on the contrary, was strongly related to more complications in the therapy process (items $1,2,6: p s \leq .01$ Table 2) and to more effort by the the rapist (item 3:p $\leq .01$, Table 2). Moreover, the efficacy of therapist effort and the ability to stop personality interference were negatively related to therapist-perceived personality pathology (items 4 and 5: ps $\leq .01$, Table 2).

The strong relation between therapist-perceived personality pathology (vas score) and complications in the Axis I therapy process did not change after entering the number of SCID-II traits as a first predictor in a series of regression equations (each time the dependent variable was one of the therapy process variables)(0.62 $\leq|B s| \leq 0.71)$. In other words, the strong relation between therapist-perceived personality pathology and complications in the Axis I therapy process was not mediated by SCID-personality pathology. 
SCID-personality pathology and therapist-perceived personality pathology and the correlation with the Axis I therapy process

\section{Therapy process wariables (100 $\mathrm{mm}$ VAS) questions answered by the therapist

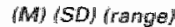

1. Is the therapy progressing with difficulty? (46)(28)(0.98)

2. Is the therapy process complicated by the personality of the patient? (48) $(28)(1-97)$

3. Do you have to exert yoursielf to help the therapy process alomg? $453+28)(4-98)$

4. Do you sere prosittive resulits of your efforts? (5.7)(20)(5-97)

5. Is it possible to adapt your attitude or approach so that the personality of the patient does not interfere with the therapy process any more? (61) $23,48 \times 99$

6. Sumscore of seven questions addressing specific problemis in the therapy process ${ }^{a}$ $(320)(128)(72-619)$

\section{SCrD il personality pathology \\ (number of traits:}

0.13

$0.27 *$
Therapist-oerceived personality pathology (100 mm WAS)

$0.60 *$

$0.70 *$

$0.66 *$

$-0.53^{* *}$

$.0 .54 *$

$p \leq .05, * * 0.07$ (two-tailled)

a The seven questions were:

1 Does the patient feel uncomfortablle in the therapy sessions?

2 Is the contact between you and this patient characterized by a tense atmosphere?

3 is the contact between you and this patient detached?

4 is the patient active and selfacting in the therapy?

5 Does the patient tolerate strong emotions that are raised by the therapeutic method?

6 Does the patient hold on to irrational thinking with respect to the Axis I complaints?

7 Is the patient demoralized albout the possibility or capacity to improve with respect to Axis I complaints?

The Impact of Personality Pathology on the Axis I Therapy Process: Specific Personality Pathology

Avoidant, dependent, obsessive compulsive, passive aggressive, paranoid, schizotypal and borderline SCID-II traitscores were evaluated in a series of multiple regression analyses with one of the therapy process variables as the criterion variable in each analysis. The traitscores of other personality categories were not included in the analyses because of their low illness rate (less than 10 cases had two or more traits). The alpha to enter was set at .05. Passive aggressive and paranoid traitscores were the only specific SCID-II variables that correlated with one or more Axis I therapy process variables. Passive aggressive traits were related to more specific problems (cf. item 6 , Table 2) $[B=0.25, t(2,67)=2.20, p=03]$ ). Paranoid traits were not only related to more specific problems, but also to more complication by the personality and more effort by the therapist (cf. items $6,2,3$, Table 2) $[B=0.26$, $t(2,67)=2.23, p=.02][\beta=0.31, t(1,68)=2.67, p=.01][\beta=0.34, t(1,68)=2.95, p=$ 
.004]. None of the specific SCID.II traitscores correlated significantly with a difficult therapy process, the efficacy of therapist effort or the ability to stop personality interference (cf. items 1, 4, 5, Table 2).

With regard to therapist-perceived personality pathology, avoidant, dependent, obsessive compulsive and passive aggressive evaluations were included in the multiple regression analyses. The illness base rate of the other personality categories was too low (less than 10 cases were rated as having 'traits' or the specific 'disorder'). The avoidant, obsessive compulsive and passive aggressive scores correlated with one or more Axis I therapy process variables. The avoidant score was related to a higher sumscore of specific problems (cf. item 6 , Table 2$)([B=0.25$, $t(3,66)=2.27, p=.03]$. The obsessive compulsive score was related to a higher sumscore of specific problems, more complication by the personality and more effort by the therapist. (cf. items $6,2,3$, Table 2$)[B=0.22, t(3,66)=2.03, p=.046][B=0.31, t(2$, $67)=2.90, p=.01][B=0.32, t(2,67)=2.96, p=.004]$. The passive aggressive score was related to the most Axis I therapy process variables: to a higher sumscore of specific problems, more complication by the personality, more effort by the therapist, and a more difficult therapy process (cf. items $6,2,3,1$ Table 2 ) $[B=0.26, t(3,66)=2.33, p=$ $.02][\beta=0.36, t(2,67)=3.36, p=.001][B=0.32, t(2,67)=2.97, p=.004][B=0.28, t(1$, $68)=2.44, p=.02]$. None of the specific personality pathology variables as perceived by the therapist correlated with the efficacy of therapist effort or the ability to stop personality interference (cf. items 4 and 5, Table 2)(This finding was in contrast with the general measure of personality pathology, judged by the therapist, that was associated with a decrease in the efficacy of therapist effort and the ability to stop personality interference). The dependent personality score was the only specific therapist-perceived variable that did not correlate with any of the therapy process variables.

\section{Discussion}

The impact of personality disorders on the Axis I therapy process was studied in an outpatient group. All patients received cognitive and/or behavioral therapy for an Axis I anxiety disordex (APA, 1987). In answer to the research questions formulated in the Introduction, the results can be summarized as follows:

1 Diagnostic agreement between the SCID-II interview and therapist evaluations on personality pathology was poor or only moderate. In other words, pathology diagnosed by the SCID-II was not automatically noticeable for the therapist during the Axis I therapy.

2 SCID-personality pathology was only weakly related to more complications and more therapist effort in the Axis I therapy process (as evaluated by the therapist).

3 Therapist-perceived personality pathology was strongly related to more complications and more therapist effort in the Axis I therapy process (as evaluated by the therapist).

4 SCID-personality pathology was neither related to the efficacy of therapist effort nor to the ability of the therapist to stop interference of the personality of the patient (as evaluated by the therapist). Therapist-perceived personality pathology, on the contrary, was related to less efficacy of therapist effort and to less ability to stop personality interference (as evaluated by the therapist). 
Earlier studies have already shown that concomitant personality disorders had no negative impact on the amount of improvement in the treatment of patients with divergent anxiety disorders (e.g. Dreessen et al., 1994; Dreessen et al., 1997; Mellman et al., 1992; Mersch et al., 1995; Noyes et al., 1990; Sanderson et al., 1994; Van Velzen et al., 1997). The parallel improvement found suggests that personality disorders did not complicate the Axis I therapy process to a degree that was insurmountable. Results of the present study corroborate this interpretation: Axis II pathology, assessed by means of a semistructured interview administered by an independent rater, had no excessive, insurmountable negative impact on the therapy process in the cognitive and behavioral treatment of Axis I anxiety disorders.

With regard to specific personality pathology, the paranoid traitscore was the only specific SCID-II variable that correlated negatively with more than one Axis I therapy process variable (Note that some specific SCID-II traitscores could not be studied). However, the paranoid SCID-II score did not correlate with efficacy of therapist effort or with the ability to stop personality interference. This indicates that complications resulting from paranoid SCID-II traits were surmountable. The results found with the paranoid SCID-II traits need to be replicated and larger-scale studies are needed to investigate the impact of more specific personality disorder variables on the therapy process in treating Axis I anxiety disorders. This is particularly so as there is growing evidence that different personality disorders or traits can have divergent effects on treatment outcome of Axis I anxiety disorders (see review by Dreessen \& Amtz, 1998-a).

With regard to therapist-perceived personality pathology, it was striking that the dependent personality score was the only variable that was not related to the Axis I therapy process (Note, again, that some specific personality variables could not be studied). Correspondingly, Chambless and colleagues (1992) found some indications that dependent personality disorder predicted an even better outcome in the treatment of panic disorder. To our knowledge, no study has ever demonstrated a negative impact of dependent personality pathology on treatment outcome for Axis I anxiety disorders (see review by Dreessen \& Arntz, 1998-a: e.g. Dreessen et al., 1997; Hoffart \& Martinsen, 1993; Steketee, 1990). Possibly, the patient with a dependent personality is very docile and compliant which might turn out to be advantageous in cognitive behavioral therapies for Axis I disorders.

As a comment on the present study, several issues should be noted. First, caution is required before generalizing the results to Axis I disorders other than anxiety disorders, or to treatments other than cognitive behavioral treatments. The type and severity of personality disorders that concur with other Axis I disorders may differ from those that concur with anxiety disorders. With respect to type of therapy, cognitive-behavioral therapy distinguishes itself from other approaches such as psychodynamic psychotherapy or psychosocial counseling, in that it is highly structured, prescriptive and it focuses on manifest symptoms with a clear rationale. Possibly, some of these characteristics facilitate working with Axis I disordered patients who have concomitant personality disorders.

Second, lumping anxiety disorders together was used as a strategy to obtain an adequate sample size. We think this strategy is permissible, because treatment outcome has been found to be unaffected by concomitant personality disorders in 
various types of anxiety disorders (e.g. Dreessen et al., 1994; Dreessen et al., 1997; Mellman et a1., 1992; Mersch et al, 1995; Noyes et al., 1990; Sanderson et al., 1994; Van Velzen et al., 1997).

A third comment concerns the way the Axis I therapy process was assessed. The process measure consisted of therapists' reports, and there was no independent confirmation of these perceptions. Furthermore, the Axis I therapy process was assessed only once (between session 6 and session 7). It would be interesting to find out whether results are similar if independent process measures are used or if the process is evaluated later on in therapy.

Finally, as mentioned in the summary of results, therapist-perceived personality pathology was found to be very different from personality pathology based on the independent interviews. The very thigh positive correlations between therapistperceived personality pathology and complications in the Axis I therapy process suggest that therapists rushed to premature conclusions in attributing failure in the Axis I therapy process to assumed concomitant personality pathology. On the other hand, one could argue that, compared to the independent raters, therapists made more valid personality disorder diagnoses in the course of therapy, because they had more or different knowledge of the patient's behavior, thoughts and feelings. It is important to bear in mind that the field of personality disorders is still struggling with both the validity of the personality disorder construct and the validity of the personality disorder assessment (Shea, 1992). Therefore, we should not assume too quickly that the SCID-II diagnoses are valid and the therapist evaluations of the personality pathology are invalid. It may turn out to be the other way around: SCID-n diagnoses being invalid and therapist evaluations being valid. Or, both the SCID-rI and therapist evaluations may turn out to be invalid. Lastly, both SCID-n and therapist evaluations may turn out to be valid even though they are different, because they refer to different aspects of the same construct.

Whatever the case, SCID-II pathology was not related to severe complications in Axis I therapy process and therefore the present results indicate an important starting point for clinicians if the Axis I therapy process gets complicated: besides personality pathology they should also consider other causes of complications, such as severity of the Axis I disorder, the impact of concomitant Axis I disorders, neinforcers of the Axis $r$ problem behavior, concomitant psychosocial and environmental problems, and of course their own method of working. Fortunately, personality disorder diagnoses are less stigmatizing than they used to be and treatment possibilities are seriously considered nowadays.

In conclusion, this study shows that concomitant personality pathology diagnosed before the start of treatment by a semistructured interview, namely the SCID-1I, had no excessively and insurmountably negative impact on the therapy process (as perceived by the therapist) in the cognitive and behavioral treatment of Axis I anxilety disorders. These findings on therapy process are consistent with previously reported findings indicating that personality pathology, as assessed by independent semistructured interviews, had no negative impact on therapy autcome of Axis I anxiety disorders. Furthermore, the present results suggest that the diagnosis of a personality disorder should not be assumed to be synonymous with difficult Axis 1 cases or as an excuse to stop investing energy in the treatment of Axis 1 anxiety disorders. 


\section{note}

1 Accepted for publication pending revision: Dreessen, L. \& Arntz, A. (1998-c).

Personality disorders have no excessively negative impact on the therapy process in the cognitive and behavioral treatment of Axis I anxiety disorders. Civical Psychology and Psychotherapy. 



\section{The impact of personality disorders on treatment outcome of anxiety disorders: best-evidence synthesis ${ }^{1}$}

\section{Summary}

Thirty-five studies were traced addressing the impact of comorbid personality disorders on treatment outcome of anxiety disonders. The conelusions of this review are based on the best-evidence procedure. We used two selection criteria that studies had to meet to be included in the present review The study design had to be prospective and a (semi)structured interview had to be used for the assessment of personality disorders. From the fifteen selected studies it cannot be concluded that, in general, personality disorders affect treatment outcome for anxiety disonders negatively. We can only suspect that some personality disorders may have an adverse impact upon treatment outcome in anxiety disorders, and they should be investigated further. For the time being, in clinical practice one has to be cautious in relating personality disorders to less well treatment outcome in Axis I anxiety disorders.

\section{Introduction}

Personality disorders refer to enduring patterns of behavior and inner experience that deviate markedly from the expectations of the individual's culture. These patterns are pervasive and inflexible, have their onset in adolescence or early adulthood, are stable over time, and lead to clinically significant distress or impairment (American Psychiatric Association, 1994, p. 629). Clinicians are quick to presume that treatment of Axis I disorders is complicated in patients with a concomitant personality disorder. Moreover, therapeutic failure is often attributed to an assumed concomitant personality disorder or personality pathology. Previously published review reports (Reich \& Green, 1991; Reich \& Vasile, 1993) have described 38 studies evaluating the impact of personality pathology on the treatment outcome of Axis I disorders (depressive disonders, anxiety disorders, substance abuse and dependence). In line with the clinical lore, these rewiew reports concluded that personality pathology adversely affects treatment outcome of Axis 1 disorders. However, results of recent studies in the field of anxiety disorders are not in line with this general conclusion (e.g. Dreessien, Arntz, Luthels, \& Sallaerts, 1994; Dreessen, Hoekstra, \& Arntz, 1997; Mellman, Leverich, Hauser, Kramlinger, Post, \& Uhde, 1992; Mersch, Jansen, \& Arntz, 1995; Sanderson, Beck, \& McGinn, 1994: Van Velzen, Emmelkamp, Scholing, \& Luteijn, 1995). The present review aims to answer the question of whether or not one can continue to conclude that, 
in general, personality disorders affect treatment outcome of anxiety disorders negatively.

To our knowledge, since 1965, 35 studies have been published or presented at international congresses addressing the effect of personality pathology on treatment outcome of anxiety disorders. Pertinent study characteristics are presented in Appendix 1. Panic disorder has been studied most frequently: 16 studies were traced. Ten studies were traced with regard to obsessive compulsive disorder. Only a few studies focused on social phobia, generalized anxiety disorder or posttraumatic stress disorder. In two studies, anxiety disorders were lumped together. Results of the 35 studies are inconsistent within and across studies: 25 studies reported a negative effect of personality disorder variables, 25 studies reported no effect, and 5 studies reported positive effects. None of the 11 different personality disorders is consistently found to affect treatment outcome negatively (Appendix 1). Also no specific anxiety disorder attracts attention regarding consistent treatment interference by concomitant personality disorders. Hoping to find more consistent results, we reviewed only the best-designed studies available by using a procedure known as the 'best-evidence synthesis' (Slavin, 1995). Two inclusion criteria were used to select the studies. First, the study had to have a prospective design. Second, personality disorders had to be assessed using a (semi)structured interview. Arguments for these criteria are discussed below.

\section{Best-evidence criteria}

The first selection criterion demands that the study has a prospective design. In several studies, personality pathology was diagnosed retrospectively (after the Axis I treatment) by the therapist or another rater (Kringlen, 1965; Lo, 1967; Mancuso, Townsend, \& Mercante, 1993; Minichiello, Baer, \& Jenike, 1987; Turner, 1987; Vaughan \& Beech, 1985). Raters in these studies were either not blind for the Axis I treatment outcome or no mention was made of being blind. A retrospective diagnosis of personality disorders by raters not being blind for the Axis I treatment outcome might be confounded. If an Axis I therapy with a particular patient is known to be a failure, it might cause the rater to diagnose more personality pathology than if the rater is blind to the Axis I treatment outcome. Even if the rater is kept blind for the Axis I treatment outcome in the first instance, the diagnosis of personality pathology might still be confounded, because the patient is never blind to his or her own treatment progress. The argument that therapists know their patients best and, therefore, their judgment is more valid is, in our view, outweighed by the argument that retrospective diagnoses will be confounded as a result of the patient's amount of progress in therapy. Personality disorders should be defined by other criteria than by failure in the Axis I therapy. Data of a recent study (Dreessen \& $A r n t z, 1998-C)$ support this supposition. Therapist judgment on personality pathology correlated highly positively with complications of the therapy process in the treatment of anxiety disorders. Personality pathology assessed before start of therapy by an independent rater using a semistructured interview correlated only low with complications in the therapy process. These results suggest that therapists attribute difficulties in the Axis I therapy to the personality of the patient. 
The second methodological selection criterion concerns the type of instrument that is used for assessment of personality disorders. There are two types of instruments: self-report questionnaires and interviews [(semi)structured and unstructured]. Regardless of type, measurement instruments for personality disorders are sensitive to state factors like anxiety and depression which co-occur with or are central to Axis I disorders (Hirschfield et al., 1983; Pilkonis, Heape, Ruddy, \& Serrao, 1991; Reich, Noyes, Coryell, \& Hirschfield, 1986). Because patients are most likely to be assessed for personality disorders when an Axis I disorder is present, it is of importance to control the influence of state factors as much as possible. In our opinion the influence of state factors is more under control in interviews than in self-report questionnaires (see also: Widiger \& Frances, 1987, p. 67; Zimmerman, 1994, p. 237). Trained clinical interviewers should be more capable than the patient of separating state from trait factors. The sensitivity of self-report questionnaires to states such as anxiety and depressive mood might explain the fact that self-report questionnaires report more personality pathology than clinical interviews (Edell, 1984; Hurt, Hyler, Frances, Clarkin, \& Brent, 1984; Reich \& Noyes, 1987; Renneberg, Chambless, Dowdall, Fauerbach, \& Gracely, 1992). Though some have expressed concerns that clinical interviews might lead to underdiagnosis of personality pathology (Widiger \& Frances, 1987), the problem of underreport by interviewers is more likely to occur in unstructured interviews than in (semi)structured interviews, in which every single trait is addressed, regardless. of the answers to previous questions. Patients may exaggerate pathology and clinicians may understate it, but it is clear that trained interviewers who use (semi)structured interviews, have more potential to eliminate their response bias. Therefore, we argue that (semi)structured interviews are more valid than unstructured interviews and self-report questionnaires. Many studies were not included in the present review because assessment was done either by unstructured interviews ( 6 studies, see Appendix 1 ) or by self-report questionnaires (12 studies, see Appendix 1).

\section{Studies selected concerning the effect of personality disorders on the treatment outcome of anxiety disorders}

Using the two selection criteria, only 15 out of 35 studies were further considered (see Appendix 1). They will be discussed below. The studies are grouped according to the type of anxiety disorder that was treated in the study. Psychological as well as pharmacological treatments were evaluated in the selected studies. In reviewing the selected studies, two questions will be addressed. First, do patients with a personality disorder respond less to treatment compared to patients without any personality disorder (i.e. differences in pretest scores are taken into account in the analyses)? Second, do patients with a personality disorder have more severe complaints at the end of the treatment that was evaluated (i.e. end-state functioning) than the patients without a personality disorder?

\section{Panic Disorder}

With regard to the effect of personality disorders on the treatment outcome of panic disorder, eight out of the sixteen studies met the best-evidence criteria. Three 
concerned pharmacotherapy (Black, Wesner, Gabel, Bowers, \& Monahan, 1994; Green \& Curtis, 1988; Reich, 1988), wo concerned cognitive and/or behaviorall therapy (Black et al, 1994; Dreessen, Arntz, Luttels, \& Sallaerts, 1994, Study 1; Chambless, Renneberg, Fydrich, Goldstein, \& Gracely, 1995), one concerned behavioral and psychodynamic group therapy combined (Hoffart \& Martinsen, 1993), and two concerned therapy given as usual (naturalistic therapy; Mellman et al, 1992; Noyes et al., 1990\%.

Green and Curtis (1988) used the Structured Clinical Interview for DSM-III-R Personality Disorders (SCID-II; Spitzer, Williams, Gibbon, \& First, 1990) and found that the presence of any SCID-II personality disorder was related to relapse after drug withdrawal in a pharmaco treatment trial with imipramine and alprazolam $(n=25,5$ drop-outs not included). Relapse was defined as less than $51 \%$ decrease in pretest complaints. Avoidant personality disorder was diagnosed most frequently and discriminated between relapsers and non-relapsers. In fact, base rates of other specific personality disorders were too low for conducting reliable analyses. Of all individual SCID-III traits, three avoidant and one narcissistic trait discriminated between relapsers and non-relapsers. Notice that 113 tests were done in evaluating the individual. SCID-II traits. The potential for a type I error is enormous. Replication is needed. Furthermore, it should be noted that the proportional change in complaints from pre- to posttest was used to define relapsers. If improvement is expressed as proportional change with respect to pretest scores, improvement of patients with personality pathology may be obscured if they have substantially higher pretest scores than patients without personality pathology. Their improvement may be less when expressed as percentage of changes, but more when expressed as raw change scores. For instance, a decrease of $50 \%$ with a high pretest score is a much larger decrease than a decrease of $50 \%$ with a low pretest score (Dreessen et al., 1994). In the study of Green and Curtis (1988), no information was given on pretest differences between relapsers and non-relapsers and between patients with and without personality pathology. Possibly, the avoidant personality disorder would not have predicted relapse if raw change scores had been analyzed in the Green and Curtis (1988) study. Five patients dropped out of therapy. They did not differ from the completers as far as the incidence of personality disorder diagnoses was concerned. The effect of personality disorders on end-state functioning (i.e. not taking into account pretest scores) was not evaluated in this study.

Reich (1988) presented data on 49 panic patients ( 3 drop-outs not included) who received alprazolam or diazepam. Treatment outcome was defined as proportional change before taper of medication. Personality disorders were measured by means of self-report questionnaires and by means of the Structured Interview for DSM-II Personality Disorders (SIDP; Stangl, Pfohl, Zimmerman, Bowers, \& Corenthal, 1985). With regard to the SIDP-diagnoses, it was found that patients with a personality disorder of the dramatic cluster (bordenline, narcissistic, histrionic or antisocial) had lower proportional change scores in the number of situational panic attacks than patients without a personality disorder of that cluster. The other outcome measures were not affected negatively. The SIDP anxious and odd cluster were not related to treatment outcome. Results of this study show some evidence for a negative effect of the dramatic cluster. Note, again, that 
proportional change scores were used, which might obscure parallel improvement of patients with and without a personality disorder of the dramatic cluster. The effect of personality disorders on end-state functioning (i.e. not taking into account pretest scores) was not evaluated in this study.

Dreessen et al. (1994, Study 1) reported on the effect of SCID-II personality pathology on treatment outcome to standardized individual cognitive-behavioral therapy. Treatment outcome was defined as change from pretests to later tests. In a group of 31 panic patients ( 4 drop-outs not included) it was found that patients with one or more personality disorders improved parallel to patients without a personality disorder. There were no differences in changes from pretest to later tests between groups (six-months follow-up included). It should be noted that personality disorders were lumped together in order to obtain adequate personality disorder base rates. Since it cannot be presumed beforehand that any personality disorder will affect treatment outcome in the same direction, one should be careful not to generalize these results to every individual DSM-personality disorder. The total number of positive SCID-II traits was also not related to treatment outcome. Stepwise regression analyses of specific personality traits using one composite outcome measure, revealed that only obsessive compulsive personality traits were related negatively to treatment outcome. Surprisingly, borderline traits predicted better outcome, but only at the six-months follow-up test. As far as the four dropouts were concerned, only one had a SCID-II personality disorder. This patient ended. treatment early but successfully. The effect of personality disorders on end-state functioning (i.e. not taking into account pretest scores) was not evaluated in this study. There was some evidence for increased Axis I psychopathology at the start of treatment in patients with a concomitant personality disorder.

Chambless et al. (1995) studied the effect of the SCID-II avoidant traitscore on the outcome of a standardized intensive behaviorally based group therapy for panic disorder with agoraphobia ( $n=49,2$ drop-outs not included). Pretest scores was the first predictor in the regression analyses on end-state scores. They found that a high avoidant traitscore was unrelated to immediate treatment outcome, but at a six-months follow-up they found that it was related to less improvement in the frequency of panic attacks. Notably, avoidant traitscore did not affect improvement of the avoidance related to the panic disorder. Both patients that dropped out of the study had one or more personality disorders. The effect of personality disorders on end-state functioning (i.e. not taking into account pretest scores) was not evaluated in this study.

In the Hoffart \& Martinsen (1993) study, panic patients with agoraphobia $(n=$ 36) received combined behavioral and psychodynamic group therapy in a standardized program. The effects of the SCID-II avoidant, dependent and paranoid personality disorder on one anxiety measure were analyzed by means of a regression analysis. Pretest scores were forced to enter first among the independent variables. None of the personality disorders predicted immediate outcome. Avoidant personality disorder predicted a less favorable change in outcome at the 1-year follow-up test. Personality disorder characteristics of the drop-outs were not addressed. The effect of personality disorders on end-state functioning (i.e. not taking into account pretest scores) was not evaluated in this study.

Black et al. (1994) studied treatment response in 66 patients with panic dis- 
order who had completed 3 weeks of treatment with cognitive therapy, fluvoxamine, or placebo pharmacotherapy. Recovery at week 4 was the dependent variable. Recovery (in $44 \%$ of the patients) was defined as either the absence of panic attacks (end-state functioning) or a Clinical Global Improvement score of 'very much' or 'much' improved. Week 4 was selected as the comparison point in order to minimize the influence of the number of patients who dropped out by week 8 , the end of the trial. Personality disorders assessed by the Structured Intervicw for DSM-MIR Personality Disorders (SIDP; Stangl et al., 1985) did not emerge as a predictor for treatment outcome (the presence of one or more personality disorders assessed by a self-report questionnaire was a negative predictor to outcome, indeed, in this study).

Finally, in two studies (Mellman et al., 1992; Noyes et al., 1990), panic patients were treated naturalistically (treatment as usual). In the Noyes et al. (1990) study, 89 patients with panic disorder were followed up after 3 years, starting from the beginning of therapy. When treatment outcome was defined as proportional change scores, the presence of any SIDP personality disorder and the total number of SIDP traits did not affect long-term treatment outcome. When treatment outcome was defined as end-state functioning, the presence of any personality disorder predicted. more severe anxiety symptoms. In this study, panic patients with personality disorders had a more severe symptom pattern at baseline, which was a strong predictor of end-state functioning at the 3-year follow-up. Personality disorder characteristics of the eight patients who dropped out of the study were not addressed.

Mellman et al. (1992) found that there were no differences in amount of improvement (change scores on anxiety and depressive symptoms) between panic disordered inpatients with and without a personality disorder. The sample size of this study was small $(n=23)$ and, therefore, different personality disorders were lumped together. The naturalistic treatment consisted of trials of medication, individual and group therapy sessions and milieu activities. No information was given on any drop-outs. The effect of personality disorders on end-state functioning (i.e. not taking into account pretest scores) was not evaluated in this study.

To summarize, most studies on panic disorder took into account pretest scores in evaluating the effect of personality disorders on treatment outcome and reported mixed results (negative and no effects of personality disorder variables and even a positive effect). Overall, the presence of one or mone personality disorders, irrespective of type, does not seem to significantly affect treatment outcome. With regard to specific personality disorder categories, avoidant personality disorder appears to be associated with a less favorable outcome in the long term (not at immediate outcome), however, this finding is not found consistently across studies (results were not replicated in the Dreessen et al. 1994 study when analyzing specific personality traits) and it is not found consistently for different panic disorder measurements (Chambless et al., 1995). Negative results with the dramatic cluster personality disorders (based on proportional change scores) and obsessive compulsive traits were found in one study and need to be replicated in panic patients before more final conclusions can be drawn. The same holds for the positive effect found with borderline traits. Other specific personality disorder categories were found to have no impact on treatment outcome, but these results also need to be replicated. 
Only two studies evaluated end-state functioning in panic disordered patients with concomitant personality disorders. Short-term end-state functioning was found to be unrelated to the presence of a personality disorder (Black et al., 1994). However, in another study, the presence of a personality disorder predicted more severe anxiety symptoms in the long term (Noyes et al., 1990).

\section{Obsessive Compulsive Disorder}

With regard to the effect of personality disorders on the treatment of obsessive compulsive disorder (OCD), two out of the ten studies met the aforementioned methodological selection criteria (Baer et al., 1992; Dreessen, Hoekstra, \& Antz, 1997).

Baer et all (1992) studied 55 patients with OCD and evaluated the effect of concomitant SIDP personality disorders on the results of a 10-weeks pharmacotherapy with clomipramine before taper of medication. None of the included patients dropped out of the study. The presence of any personality disorder, irrespective of type, did not affect immediate treatment outcome (end-state functioning). Schizotypal, avoidant and borderline personality disorder, along with the total number of personality disorders diagnosed correlated to poorer treatment outcome. This was revealed in multiple regression analyses (pretest score was first forced into the regression equation). No other personality disorder had a significant correlation with treatment outcome measures. Note that no conclusion can be drawn about the schizoid, narcissistic and antisocial personality disorder because of the low positive base rate in the sample $(n \leq 1)$. The odd cluster taken as a class of personality disorder's was also a negative predictor for outcome (pretest score was used as a covariate), but the odd cluster patients also accounted for the largest number of total personality disorder diagnoses. The other clusters did not relate to treatment outcome (pretest score was not used as a covariate in evaluating the anxious cluster, because groups did not differ at pretest). Finally, all 96 SIDP traits were evaluated in exploratory analyses. "Two schizotypal criteria and one paranoid criterion were significantly related to poorer outcome. Notice that with 96 tests the potential for a type lerror is enormous. This study was part of a larger placebo-controlled drug trial in which patients with personality pathology apparent on clinical interview (not by use of the SIDP) that may have interfered with protocol compliance were excluded. Consequently, four patients were excluded for suspected schizotypal personality disorder on the unstructured clinical interview. It is tempting but dangerous to assume that the negative effect of schizotypal personality disorder would increase by including these patients in the study. It goes without saying that studies on the effect of personality disorders on treatment outcome of $A$ xis 1 disorders should not exclude patients with concomitant personality disorders beforehand.

Dreessen et al. (1997) studied 43 patients who completed a standardized cognitive behavioral treatment for their obsessive compulsive Axis I complaints. The presence of one or more personality disorders had no impact upon change from pretest to later tests. Furthermore, the effect of avoidant, dependent, obsessive compulsive, paranoid and schizotypal personality disorder diagnoses was evaluated. None of these individual personality disorders were related to immediate or longer-term treatment outcome (i.e. change scores). The effect of 
personality pathology was also studied by evaluating dimensional personality variables (the total number of personality disorder diagnoses, the total number of personality traits, and the avoidant, dependent, obsessive compulsive, paranoid, schizotypal, passive aggressive and self-defeating traitscores). None of these variables significantly predicted treatment outcome (i.e. change scores). With respect to the schizotypal personality disorder and traits there was a trend towards significance. When data from the drop-outs $(n=9)$ were included in the analyses the results remained similar. The data from the drop-outs were estimated: change scores of the drop-outs were either set at zero (no improvement) or they were calculated by subtracting the mean change score of patients in the same treatment condition from the individual pretest score of the patient who dropped out. Dropouts did not differ from study completers with regard to personality disorder characteristics. The effect of personality disorders on end-state functioning (i.e. not taking into account pretest scores) was evaluated by classifying patients as recovered or unrecovered. No differences were found between the percentages of patients with and without personality disorders that recovered.

To summarize, the presence of any personality disorder, irrespective of type, was found to be unrelated to treatment outcome in both studies (one study evaluated change scores and both studies evaluated end-state functioning). With respect to the effect of specific personality disorders (e.g. effects of the avoidant and borderline personality disorder) and the total number of diagnoses, results were contradictory. In both studies, some evidence was found for a negative influence of the schizotypal personality disorder. However, the effects were not robust.

\section{Social Phobia}

Concerning the effect of personality disorders on the treatment outcome of social phobia, three out of four studies met the best-evidence criteria (Feske, Perry, Chambless, Renneberg, \& Goldstein, 1996; Mersch, Jansen, \& Arntz, 1995; Van Velzen, Enmelkamp, Scholing, \& Luteijn, 1995).

Mersch et al. (1995) studied the effect of the presence of one or more SCID-II personality disorders on treatment outcome of a standardized cognitive behavioral treatment in 28 patients with social phobia (three-months follow-up inclusive). Patients with a concomitant personality disorder had more severe Axis I symptomatology at the beginning and end of the therapy ( 3 months follow up inclusive), but they improved as much as patients without a personality disorder. Six patients dropped out of treatment or did not respond at the follow-up. Only one of them had concomitant personality disorders.

Preliminary results found by Van Velzen et al. (1995) also indicated that the presence of one or more SCID-II personality disorders did not affect immediate treatment outcome of behavior therapy in patients who finished treatment as planned $(n=32)$. Change with treatment and end-state functioning were equal in patients with and without personality disorders. Eighteen patients dropped out of therapy. There were no differences between drop-outs and completers with regard to the presence of personality disorders. The high drop-out rate might be due to exposure in vivo exercises that had to be performed in the presence of the therapists and that could not be avoided. 
Feske et al. (1996) evaluated the effect of the SCID-II avoidant personality disorder on exposure-based treatment outcome in 48 social phobic patients. Patients with an avoidant personality disorder had higher pretest scores compared to patients without avoidant personallity disorder. From pre- to posttest, patients with an avoidant personality disorder improved less with regard to trait anxiety and self-esteem than patients without an avoidant personality disorder. However, no group differences in improvement rate were found with regard to depression, social adjustment and social phobic complaints (pretest scores were taken into account in the analyses). During the 3-months follow-up period patients with an avoidant personality disorder improved at the same rate as those without an avoidant personality disorder for all outcome measures. Although both groups improved at the same rate, patients with an avoidant personality disorder continued to have more severe symptomatology than those without an avoidant personality disorder.

To summarize, patients with or without personality disorders seem to benefit equally from cognitive and/or behavioral therapy for their social phobic complaints. One study focused on the avoidant personality disorder specifically, and found a negative influence on trait anxiety and self-esteem, but not on other outcome measures. Whereas the amount of improvement is equal in the diagnostic groups, complaints after treatment continue to be more severe in patients with personality disorders compared to patients without personality disorders (except in the Van Velzen et al. (1995) study).

\section{Generalized Anxiety Disorder}

Of the two studies that evaluated the effect of personality disorders on the treatment of generalized anxiety disorder, only the Sanderson, Beck, and McGinn, (1994) study met the best-evidence criteria. Sanderson et al. (1994) found no effect of the presence of one or more SCID-II personality disorders on the immediate treatment outcome of cognitive therapy in a group of 22 patients with generalized anxiety disorder. Change with treatment and end-state functioning were equal in patients with and without personality disorders. The number of treatment sessions was not predetermined. No report was given of the final duration of treatment for the patients with and the patients without a personality disorder. Long-term treatment outcome was not evaluated. Ten patients $(31 \%)$ attended less than six sessions and they were considered as drop-outs. The proportion of patients with a personality disorder tended to be higher in the group of drop-outs than in the group of completers.

\section{Any Anxiety Disorder}

In two studies, different types of anxiety disorders were lumped together (see Appendix 1). One of these studies met the best-evidence criteria (Dreessen et al., 1994, Study 2). In this study, 57 patients (16 drop-outs not included) received an individually tailored cognitive behavioral therapy. The total number of therapy sessions of patients with and without personality disorders did not differ. The presence of one or more SCID-II personality disorders and the total number of SCIDII personality traits had no impact on change with treatment. In a multiple regression analysis, only the total number of obsessive compulsive traits predicted 
worse outcome as far as the specific personality traits were concerned. The effect of personality disorders on end-state functioning (i.e. not taking into account pretest scores) was not evaluated in this study, but anxiety patients with a personality disorder had more severe complaints at the start of treatment. There were no differences in personality disorder characteristics between patients included in the analyses and the drop-outs.

\section{Conclusions and discussion}

Based on the best evidence available, it cannot be concluded that, in general, patients with a personality disorder respond less to treatment for their Axis I anxiety disorder compared to patients without a personality disorder. Fifteen studies on this issue yielded mixed results. Detrimental effects of personality disorders on the outcome of pharmacotherapy were reported by Green and Curtis (1988) for the avoidant personality disorder, by Reich (1988) for the dramatic cluster personality disorders, and by Baer et al. (1992) for the schizotypal, avoidant, borderline and odd cluster personality disorders. Detrimental effects of personality disorders on outcome of psychotherapy were reported by Dreessen et al. (1994) for the obsessive compulsive traitscore and by Hoffart and Martinsen (1993), Chambless et al. (1995) and Feske et all. (1996) for the avoidant personality disorder/traitscore. Besides these detrimental effects, a lot of the selected studies reported no effect of personality disorder variables on treatment outcome of anxiety disorders (Bear et al., 1992; Chambless et al., 1995; Dreessen et al., 1994; Dreessen et al., 1997; Feske et al., 1996; Hoffart \& Martinsen, 1993; Mellman et al., 1992; Mersch et al., 1995; Noyes et al., 1990; Reich, 1988; Sanderson et al., 1994; Van Velzen et al., 1995). One study reported a positive effect of a personality disorder variable on long-term treatment outcome (Dreessen et al., 1994).

The detrimental effects of personality disorders found in some studies concern only a part of the relevant outcome measures (e.g. results of Chambless et al., 1995; Feske et al., 1996 and Reich, 1988) or they were not detected consistently across studies concerning different Axis I anxiety disorders (e.g. avoidant, obsessive compulsive and schizotypal personality disorder were also found to be unrelated to treatment outcome of anxiety disorders). Mixed findings can be expected when different interviews for personality disorders are used, when different treatment modalities are evaluated across studies, when sample sizes are relatively small (the impact of personality disorders has to be very large to be detected consistently: potential for type II error), and when multiple tests are done (the impact of personality disorder variables may be found by chance: potential for type I error).

The second question addressed in this review is whether patients with a personality disorder have more severe complaints at the end of the treatment (i.e. end-state functioning) than those without a personality disorder. Again, studies yielded mixed results. Noyes et al. (1990), Mersch et al. (1995), and Feske et al. (1996) reported worse end-state functioning of anxiety patients with a personality disorder compared to patients without a personality disorder. Baer et al. (1992), Black et al. (1994), Sanderson et al. (1994), Van Velzen et al. (1995), and Dreessen et al. (1997) reported no differences in end-state functioning between patients with and without personality disorders. This discrepancy cannot be explained by type 
of anxiety disorder. When patients with personality disorders have more severe complaints at the start of treatment, it is not to be expected that they improve more and in that manner reach the same end-state functioning. Before to conclude that concomitant personality disorders impair treatment of anxiety disorders in terms of end-state functioning, longer treatment should be given to patients with concomitant personality disorders given their higher levels of pathology at start of treatment. In the Mersch et al. (1995) study, end-state functioning was evaluated after a fixed number of treatment sessions, but in the Noyes et al. (1990) study and in the Feske et al. (1996) study, the number of treatment sessions was not controlled in the follow up period of 3 years and 3 months, respectively. Nevertheless, patients with a personality disorder did not reach the end-state functioning of patients without a personality disorder. Of the five studies that reported no differences in end-state functioning, in three studies the duration of treatment was fixed (Bear et al., 1992; Black et al., 1994; Van Velzen et al., 1995), in one study the duration of treatment was not fixed (Sanderson, et al. 1994), and in one study additional treatment was given in the follow-up period, if needed (Dreessen et al., 1997). In short, whereas some studies found equal end-state functioning in patients with and without personality disorders even without additional treatment, others found unequal end-state functioning even after additional treatment. More research is needed to better understand the impact of personality disorders on end-state functioning.

To sum up, taking into account the potential for type I and type II errors, we cannot draw firm conclusions on the impact of personality disorders on treatment outcome of anxiety disorders. For the most part, personality disorders seem to have no impact upon treatment outcome. At the very least it can be concluded that there is no robust negative impact of personality disorders. Based on research so far, we can only suspect that some specific personality disorders, such as a concomitant avoidant personality disorder in patients with panic disorder or a concomitant schizotypal personality disorder in patients with obsessive compulsive disorder, affect treatment outcome of anxiety disorders negatively and these disorders should be investigated further. In any case, one should be careful in drawing firm conclusions concerning personality disorders as being negative predictors of treatment outcome in anxiety disorders.

Treatment outcome as evaluated by the majority of the selected studies concerned patients who completed the study. If patients with a concomitant personality disorder tend to select themselves out of a (standardized) treatment, it would be too optimistic to conclude that for the most part, personality disorders do not seem to impair treatment outcome in anxiety disorders. Patient who do not attend treatment sessions or who do not take the prescribed drug, cannot benefit from any therapy. In the majority of the selected studies, drop-outs are discussed with regard to their personality disorder characteristics. None of the studies give reason to suspect that patients with concomitant personality disorder tend to select themselves out of treatment.

In our opinion, the best way to study the effect of concomitant personality disorders on treatment outcome for anxiety disorders is to include data of the drop-outs in the final analyses on the treatment outcome. This procedure was followed in the study of Dreessen et al. (1997). Personality disorder variables still 
did not affect treatment outcome of patients with $O C D$ after including data of the drop-outs.

The conclusion of our present review is more nuanced and formulated in a less firm tone of voice than the conclusions in previous reviews mentioned in the introduction (Reich \& Green, 1991; Reich \& Vasile, 1993). Several reasons can be mentioned for this discrepancy. First, we evaluated only anxiety disorders, whereas the previous reviews included other Axis I disorders also, such as depressive disorders. Second, we used two a priori selection criteria, whereas in previous review studies no best-evidence restrictions were made. Third, our review includes recent studies from which data were not available at the time of the earlier reviews. Fourth, in the earlier review studies less emphasis was placed on results that were not in line with the general conclusion that personality disorders affect treatment outcome negatively.

\section{Theoretical Considerations}

In the last decade, several theoretical models have described ways in which personality disorders may complicate the therapeutic process (Beck, Freeman, \& Associates, 1990; Benjamin, 1993; Horowitz, Rosenberg, \& Bartholomew, 1993). Complications in the therapeutic process are believed to result in less well treatment outcome. However, the present review shows that there is no convincing evidence for the prevailing general idea that personality disorders have a negative impact on treatment outcome of Axis I anxiety disorders. Several explanations are possible. First of all, complications in the therapy process, if any, do not necessarilly affect final treatment outcome. Complications may be surmounted by the therapist (e.g. by adapting to the interpersonal style of the patients or by changing therapy interfering cognitions in a successful way). Second, it may be that only certain, more specific, personality disorder variables complicate the treatment of Axis I disorders. More specific variables are the separate personality disorders, or even more specifically, certain deeply ingrained (interpersonal) behaviors or beliefs. Examples of potentially interfering behaviors are the tendency to avoid strong emotions or the avoidance of interpersonal contact due to the fear of negative evaluation. Examples of potential. interfering beliefs are the assumptions that effort to change is useless or that it is better to do nothing than to make a mistake. Future research should focus on more specific a priori hypotheses based on theoretical models.

With regard to the present review one should bear in mind that personality disorders are defined as patterns of behavior and inner experience that have an onset in adolescence or early aduithood and that are stable over time (APA, 1994, p. 629). Accordingly, a personality disorder should not be diagnosed if the features occur exclusively during an episode of an Axis I disorder. This was the starting position of the present review. However, if features occur exclusively during an episode of an Axis I disorder, theoretically they could be considered as subclinical personality characteristics that flourish as a consequence of life stress. It would be very interesting to evaluate the impact of these temporary personality disorder features on treatment outcome of Axis I anxiety disorders and to find out whether the results are similar to the results found with 'real' personality disorders. 


\section{Clinical Implications}

Apart from the theoretical considerations, the general conclusion of the present review has important clinical implications. First, clinicians must not exclude patients with a comorbid personality disorder from treatment for their Axis I anxiety disorder in advance. Second, attributing therapy failure to a concomitant personality disorder should be approached with caution. Third, clinicians should be aware of their own attitude towards patients with personality disorders: the therapist' belief that patients with a concomitant personality disorder will not benefit from any therapy ${ }_{r}$ might initiate a selffulfilling prophecy. It is considered a therapeutic skill to anticipate problems to be expected in treatment. Nevertheless, clinicians must be critical of their own prejudices and concentrate on overcoming interpersonal difficulties that may arise from personality disorders.

\section{Future Directions}

We end this article by suggesting ways in which research in this field may be improved. First, more studies are needed with large sample sizes that evaluate the effects of individual personality disorders or individual traits (behavior and beliefs). Personality disorder variables that affect treatment outcome may be very specific. For example, low tolerance of strong emotions, or a deeply ingrained belief that effort to change is useless, might be sufficient to cause failures in treatment. We suggest that future research should concentrate on studying well reasoned hypotheses concerning the mechanism that causes Axis II to interfere with Axis I treatments. These hypotheses will indicate the personality disorder variables that should be investigated.

Along with the demand for new larger scale studies comes the need for more reliable and valid measurement instruments. (Semi)structured interviews for personality disorders have only a modest short-interval test-retest reliability for most of the personality disorders (Dreessen \& Arntz, 1998-b; First et al., 1995-b; Stangl et al., 1985; Zanarini, Frankenburg, Chauncey, \& Gunderson, 1987). Studies that have compared two different interviews have generally found only poor to fair agreement (Hyler, Skodol, Kellman, Oldham, \& Rosnick, 1990; O'Boyle \& Self, 1990; Oldham et al., 1992; Skodol, Oldham, Rosnick, Kellman, \& Hyler, 1991). In other words, (semi)structured interviews for personality disorders are not in agreement as to what constitutes a personality disorder.

Third, the best-available evidence suggests that short-term treatment outcome is not convincingly affected by the presence of categorical or dimensional personality disorder variables, which raises the important question of whether the same holds for medium and long-term treatment outcome. Several studies, but not all, address this question (Chambless et al., 1995; Dreessen et al., 1994; Dreessen et al., 1997; Feske et al., 1996; Hoffart \& Martinsen, 1993; Mersch et al., 1995; Noyes et al., 1990). The medium to long-term follow-up periods ranged from three months to three years and results were mixed within and across studies (see Appendix 1). Future studies should focus specifically on long-term treatment outcome; first, to evaluate whether patients with personality pathology maintain equal treatment gains in the long term compared to patients without personality pathology, and second, to evaluate whether they ever reach the same end-state functioning. 
Fourth, in the present review we discussed 15 studies, of which 3 were pharmacotherapy studies and of which 8 were cognitive behavioral therapy studies. In the remaining studies treatment methods were mixed or unstandardized. The pharmacotherapy studies mostly show negative effects of personality disorder variables on treatment outcome, whereas the cognitive behavioral therapy studies mostly show no effects (see Appendix 1). Many factors, such as the prevalence of specific personality disorders, are not controlled for between the studies. Therefore, one should not rush to a conclusion that is detrimental to pharmacotherapies. The possibility that the effect of personality pathology interacts with the type of intervention used for the treatment of anxiety disorders (e.g. pharmacotherapy vs. cognitive behavioral therapy) deserves further study in carefully controlled designs.

\section{note}

1. Published as: Dreessen, L. \& Arntz, A. (1998-a). The impact of personality disorders on treatment outcome of anxiety disorders: Bestevidence synthesis. Behaviour Research and Therapy. $36,483-504$ 


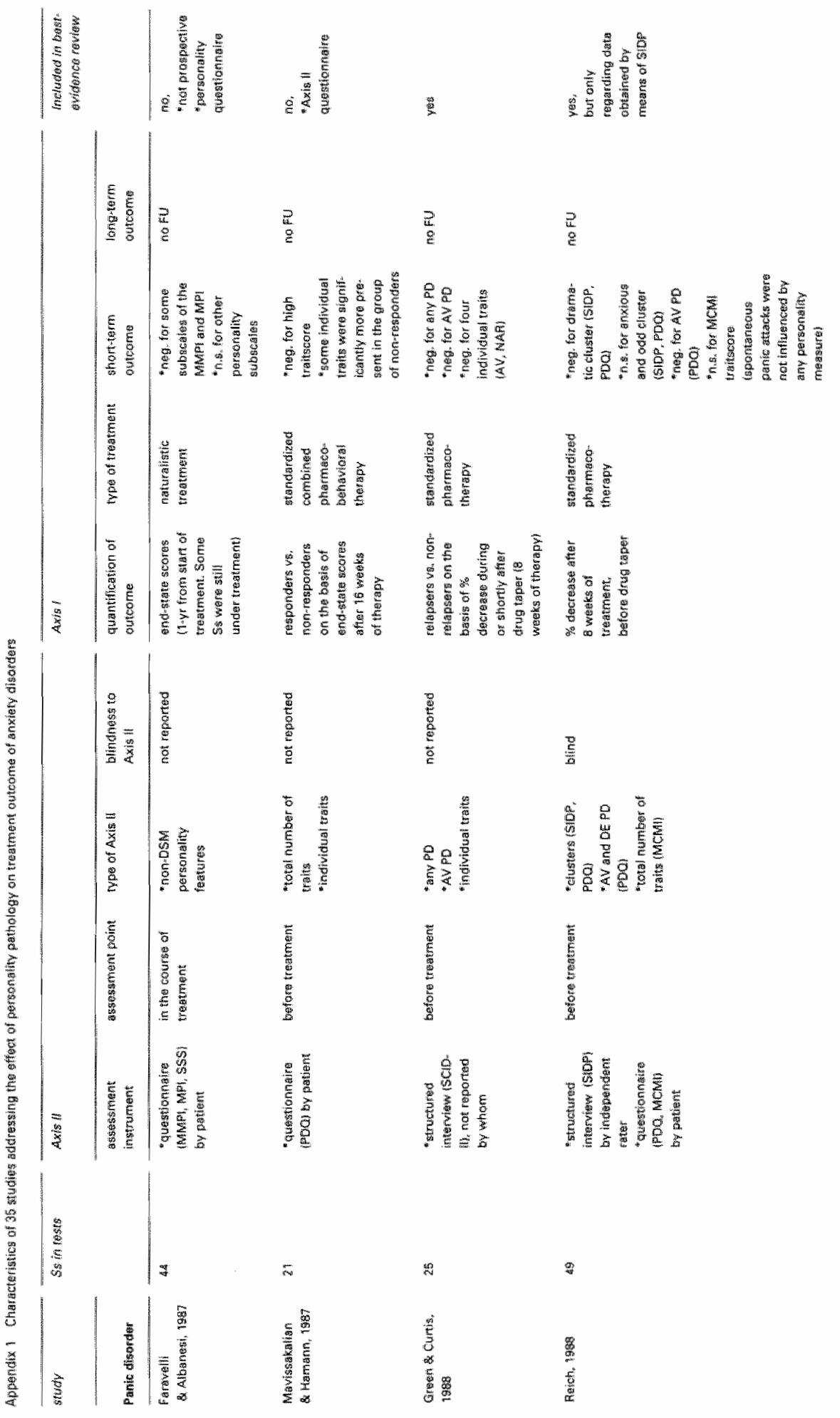




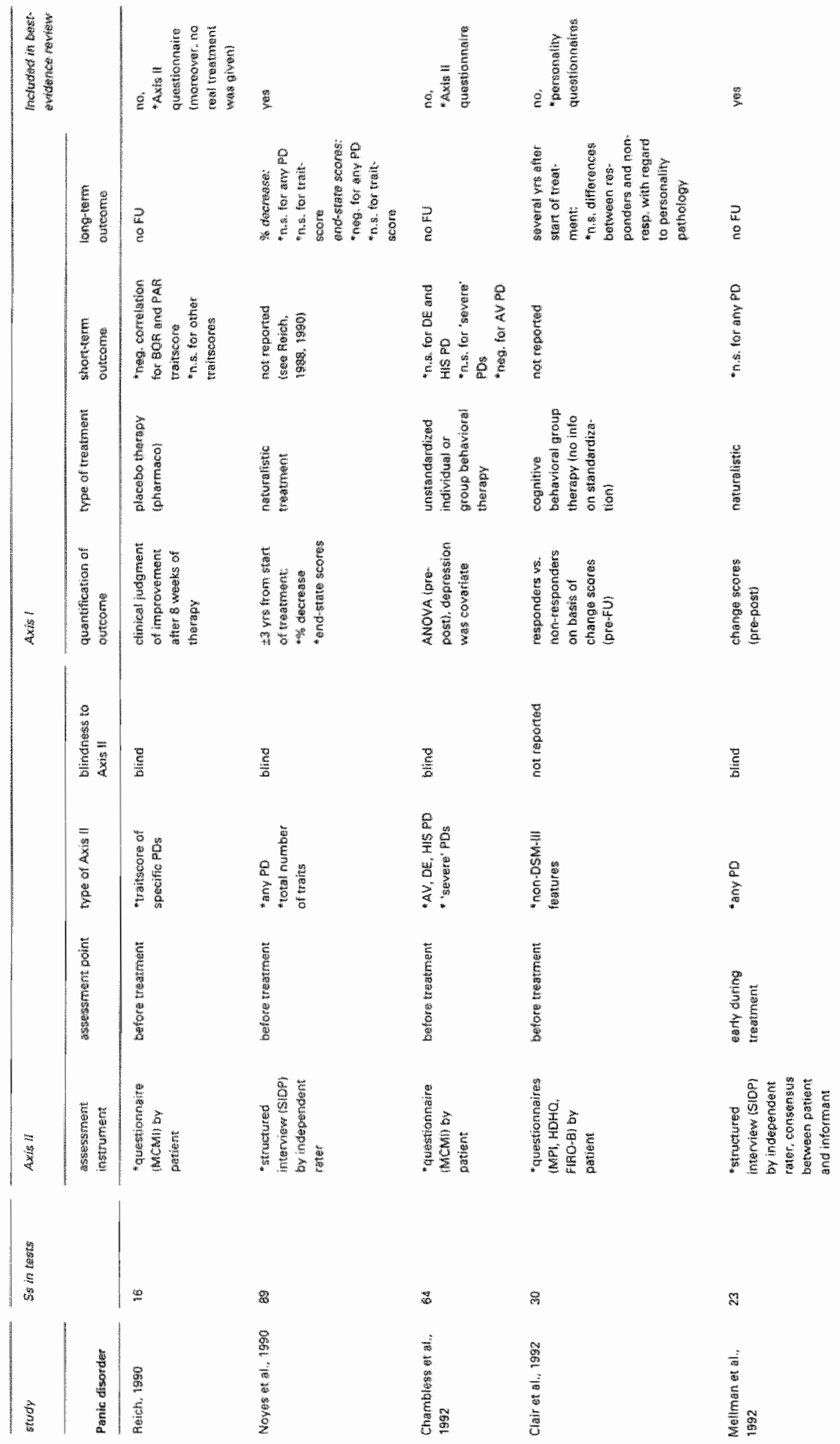




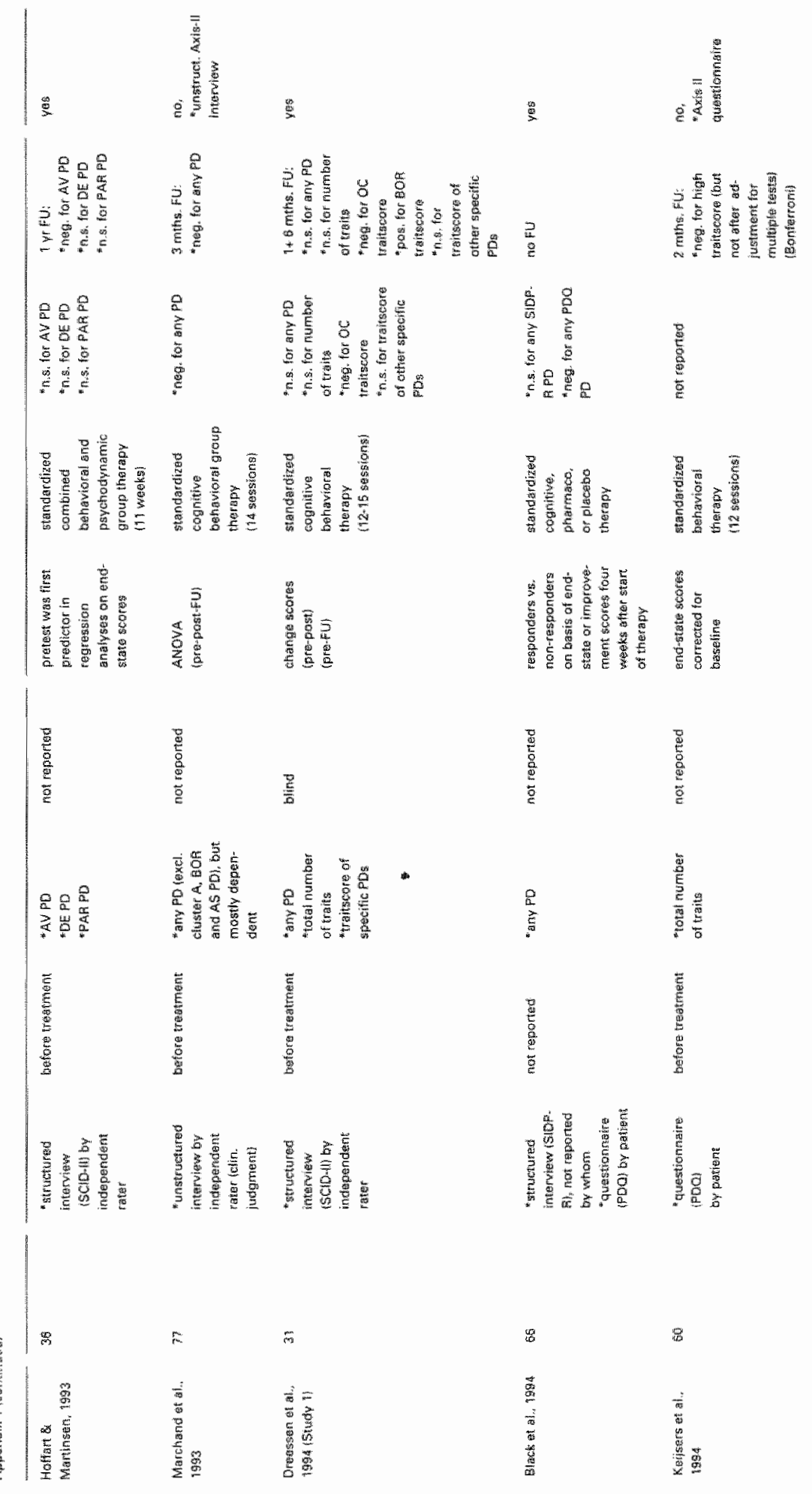




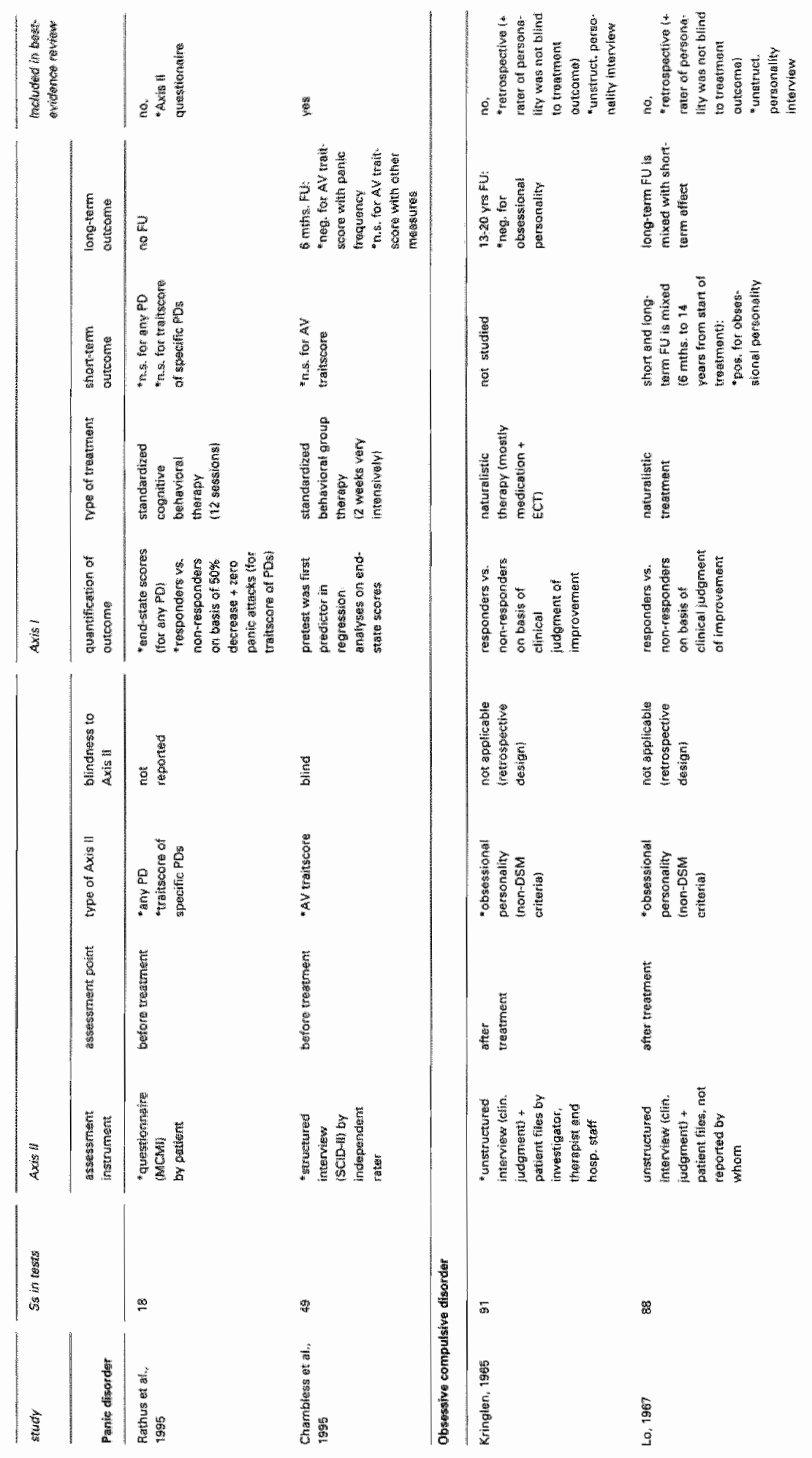




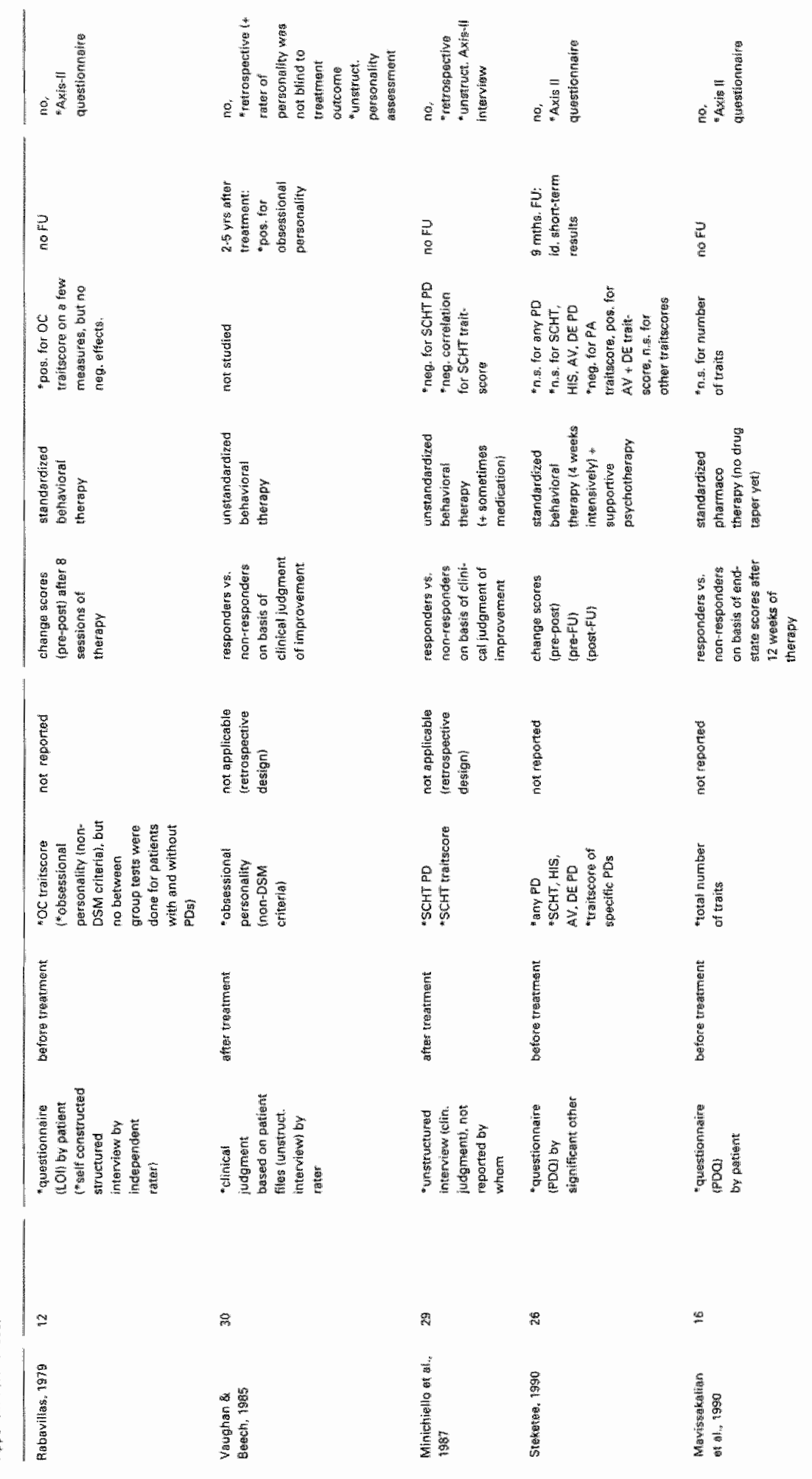


IIInm

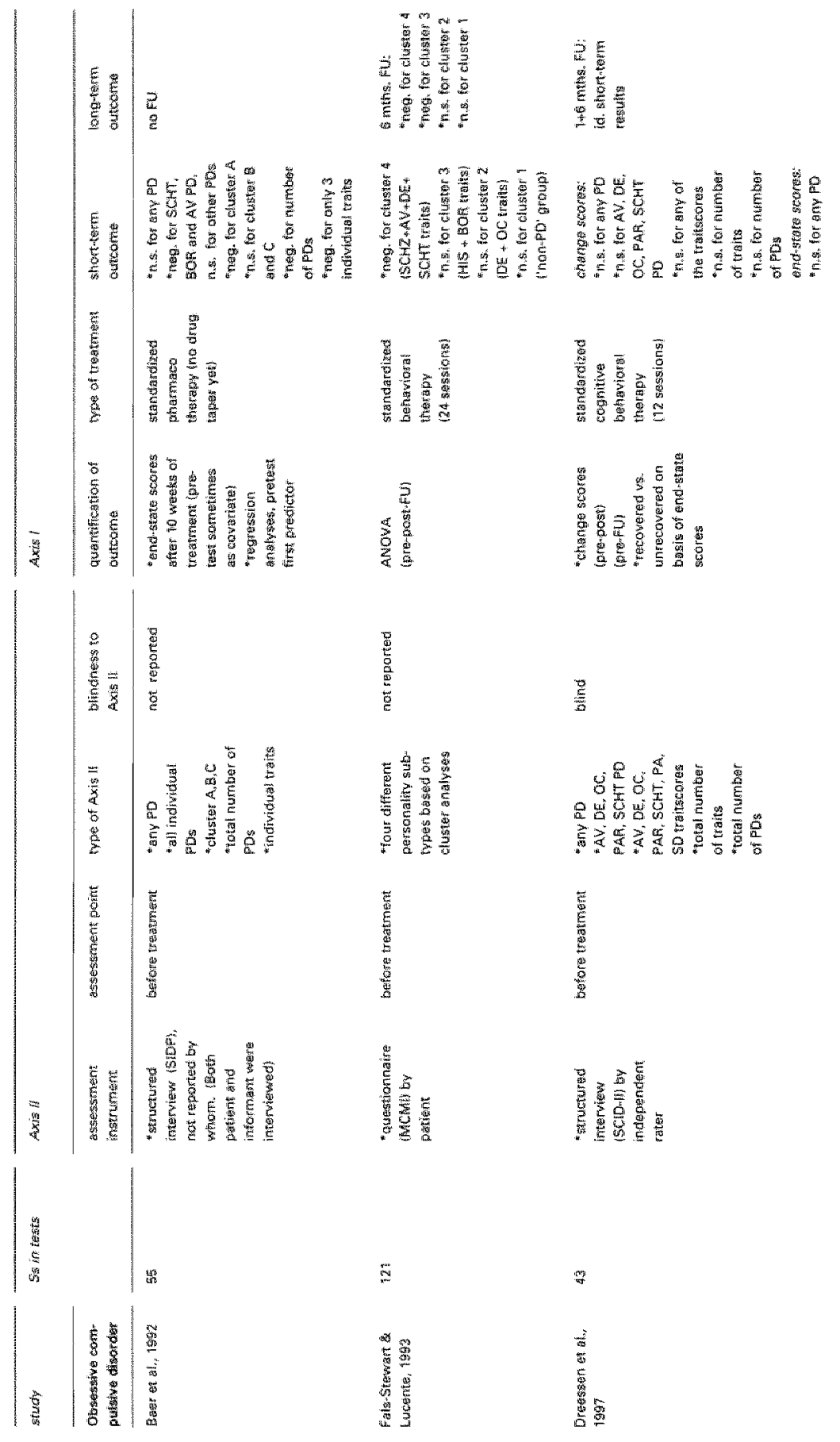




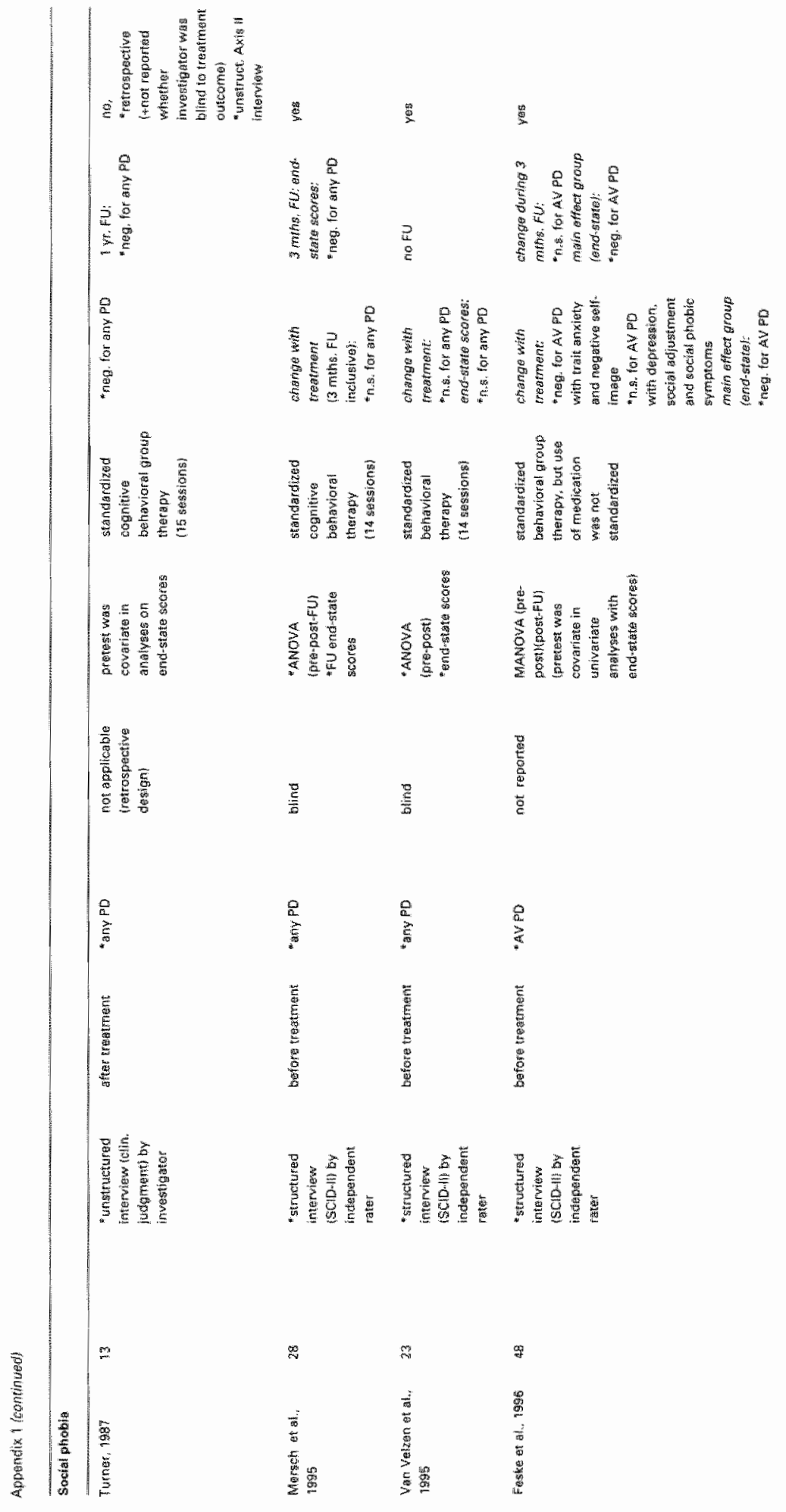




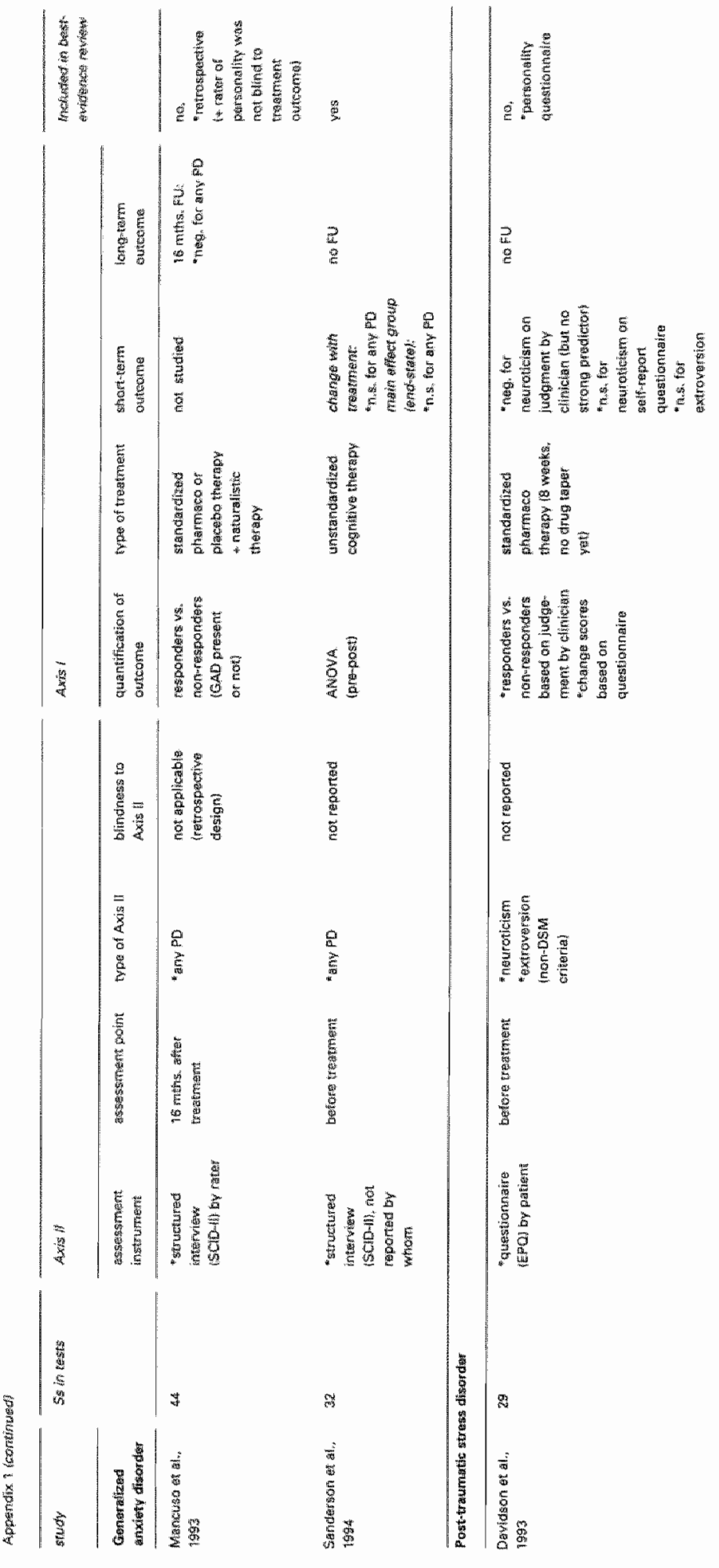




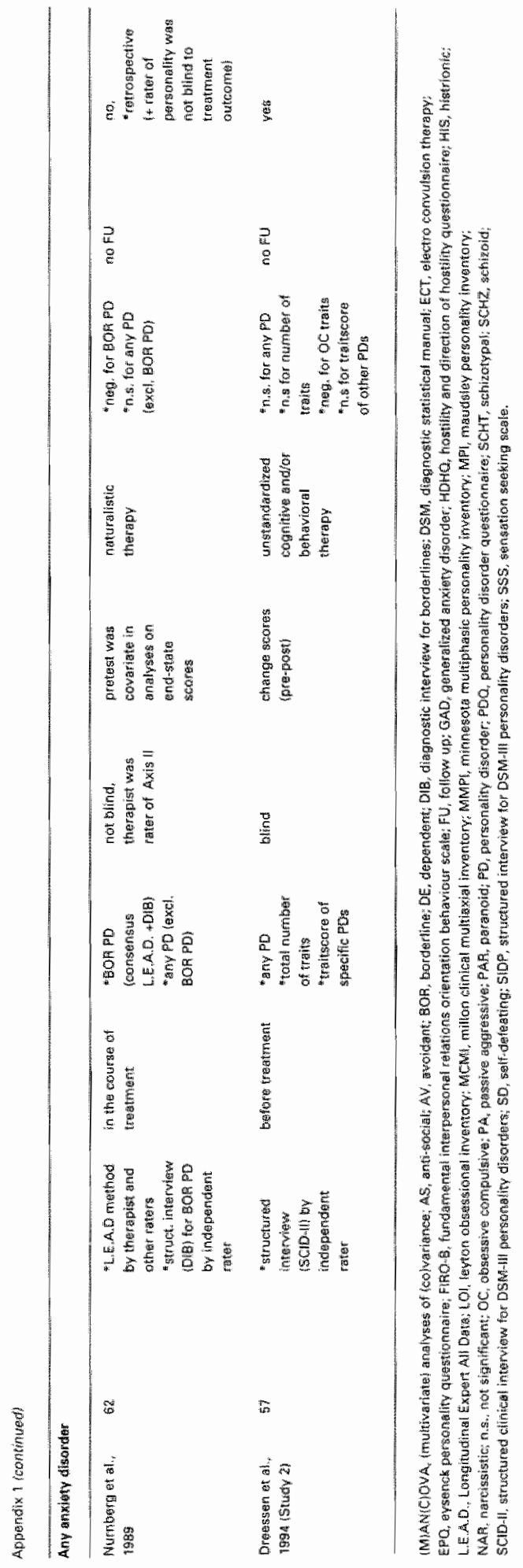




\section{Cognitive profiles of personality disorders}

In Chapter 5 , a recently developed self-report questionnaire is introduced, the socalled Personality Disorder Belief Questionnaire (PDBQ), which attempts to identify maladaptive cognitive profiles (i.e. beliefs) that correspond with the DSM personality disorders (section 5.1). Furthermore, the first study in a series of studies designed to test the cognitive model of personality disorders, is presented. It is tested whether the DSM-III-R avoidant personality disorder is characterized by biased information processing governed by typical maladaptive schemas (section 5.2). 


\section{Assessment of cognitive profiles in personality dis- orders: development of the Personality Disorder Belief Questionnaire (PDBQ)}

\section{Summary}

The background and description of the Personality Disorder Belief Questionnaine (PDBQ) is presented. The PDBQ is a self-repont questionnaire for measuring cognitive profiles of personality disorders of the Diagnostic and Statistical Manual of Mental Disorders (DSM, 4 th ed), Each subscale represents maladaptive beliefs that are hypothesized to be characteristic for one of the 12 DSM-IV personality disorders. Research on psychometric properties is ongoing Preliminary data are promising. Both in empirical research and dinical practice, the PDBQ may turn out to be a useful self-report questionnaire for the assessment of beliefs related to personality disorders.

\section{Background}

Cognitive therapy has been used with increasing frequency to treat personality disorders (PDs) (Beck, Freeman, \& Associates, 1990; Padesky, 1994; Young, 1990). In cognitive therapy of PDs it is attempted to modify underlying malladaptive cognitive structures (i.e. schemas) which are hypothesized to govern information processing and to create distress through cognitive biases and maladaptive behavioral patterns. The content of the maladaptive schemas in PDs is hypothesized to refer to generalized representations (or knowledge) of the self, others and the world (Beck et al., 1990; Young, 1990), which are mainly implicit. The explicit verbal formulations of these implicit representations are called beliefs or assumptions.

Identification of $\mathrm{PD}$ beliefs is, of course, crucial in cognitive therapy of $\mathbb{P D S}$. Besides this, identification of PD beliefs may also be useful in any other psychological or pharmacological therapy, as these beliefs are hypothesized to be operative on everyday information processing. In that way, they potentially affect any relationship or event, including therapies that focus on other problems than those directly related to PDS. A cognitive conceptualization of the personality of the patient, provides a framework in which extreme behavioral and emotional reactions can be explained. The therapist can adapt his behavior and emotional reactions to the maladaptive PD beliefs (thus, not necessarily modify the beliefs [cf. schemas], as in CT for PDS) and be more patient and understanding, which may reduce the risk of a disturbed therapy process. 
There are several methods to identify underlying PD beliefs. Beliefs can be inferred from automatic thoughts, behavior and affect (the information processing output). Furthermore, one can question the meaning of high affect events (Padesky, 1994). People can be asked what went through their mind during the high affect event. Automatic thoughts can be further explored for their deeper meaning, by asking questions like 'Suppose this is true, what would it mean to you? What would you conclude from it? What would be the worst thing for you to happen?' [the so-called downward arrow technique (Fennell, 1989)]. People can also be requested to do a series of sentence completions (e.g. I am Others are Padesky, 1994).

A more standardized way to identify maladaptive beliefs is to use belief questionnaires. To date, only Beck et al. (1990) and Young (1990) have initiated the development of such questionnaires.

Beck et al. (1990, pp. 359-363) presented a list of beliefs without a rating scale. The list contains 9 sets of 14 beliefs hypothesized to be typical for nine of the eleven DSM-III-R PDs (American Psychiatric Association, 1987). The 126-item list was used as the basis of a questionnaire, the so-called Belief Questionnaire (BQ; Beck, 1990), by adding a 7-point scale with each belief ranging from totally disagree (1) to totally agree (7). Although the schema list in Beck et al.'s book has been mentioned by various authors (e.g. Padesky, 1994; Fydrich, Schmitz, Hennch, \& Bodem, 1996 ${ }^{1}$ ), there has been only one report on the BQ (Trull, Goodwin, Schopp, Hillenbrand, \& Schuster, 1993). Trull et al.'s study reported on psychometric properties of the BQ. Results indicated high internal consistency and test-retest reliability for the $B Q$ Scales. Validity results, however, were not so strong.

The BQ provides no items for the borderline and the schizotypall PDs because it was believed that these two disorders do not involve a typical set of beliefs (Beck et al., 1990). Moreover, no items are provided for the depressive $\mathrm{PD}$, because this disorder was introduced only in the DSM IV in 1994 (APA, 1994).

Young (1990) has developed the so-called Schema Questionnaire ${ }^{2}$ (SQ), which is a 205-item self-report questionnaire. The SQ does not start from the DSM PDS, but from 16 different themes, such as self-sacrifice, undesirability, or unrelenting standards, which are hypothesized to be relevant to PDs. The items are rated using a 6-point scale ranging from completely untrue for me (1) to describes me perfectly (6) (for promising psychometric properties see Schmidt, Joiner, Young, \& Telch, 1995).

In conclusion, beliefs play a central role in cognitive conceptualizations of PDs. However, standardized cognitive measures of PDS are scarce and incomplete.

This paper reports on a recently developed Dutch self-report questionnaire for measuring cognitive profiles of PDs: the Personality Disorder Belief Questionnaire (PDBQ; Dreessen, Arntz, \& Weertman, 1996). Each subscale represents maladaptive beliefs that are hypothesized to be characteristic for the PDs included in the DSM-IV (APA, 1994). A final version of the PDBQ has not been published yet. A draft version may be obtained by contacting the author. 


\section{Features of the instrument}

\section{Comerage.}

The PDBQ consists of 12 subscales, corresponding with the 12 PDs included in the DSM-IV classification or suggested to be included in the future classification (APA, 1994): 1) Avoidant, 2) Dependent, 3) Obsessive compulsive, 4) Passive aggressive, 5) Paranoid, 6) Schizotypal, 7) Schizoid, 8) Histrionic,9) Narcissistic, 10) Borderline, 11) Antisocial, 12) Depressive. Each PD is represented by 20 general beliefs, partly formulated on the basis of Beck et al. ${ }^{3}$ (1990, pp. 359-363, with permission) and partly hypothesized by the constructors based upon theoretical considerations and their clinical experience with patients with PDS. The PDBQ is intended to be a trait measure. Respondents are instructed to consider the last couple of years in evaluating the beliefs. Of course, state belief scores can also easily be generated by simply changing the instructions to the respondents.

\section{Structure and Administration}

When all DSM-IV PDs are included, the questionnaire contains 240 items. Every item refers to a specific belief, which has to be rated on a $100 \mathrm{~mm}$ Visual Analogue Scale (VAS) in terms of strength of belief. The anchors are formulated as I don't believe this at all and I believe this completely. The respondent has to place a vertical mark on the VASs. Appendix 1 shows some examples.

The instrument begins with a written instruction how to fill out the questionnaire. It is explained that people can believe in statements concerning themselves, others or the world to an increasing degree. Two examples ane given to illustrate dimensional ratings. The respondent is instructed to convey his or her actual opinion, and not how much they would like to believe the item or how much they think they should believe the item. It is stressed to consider the last couple of years in rating the beliefs. Next, the 240 randomized beliefs have to be rated. It is also possible to administer only those sections on PDs of particular interest. The PDBQ can be administered individually or in groups with adequate privacy. Usually, it takes the respondent about 40 minutes to fill out the entire questionnaire. A computerized version has recently been developed.

In developing the $P D B Q$ we doubted whether naive respondents were capable of recognizing and rating their underlying beliefs. It turned out that respondents (monpatients and outpatients) had no difficulty in completing the PDBQ and that they were intrigued by being confronted with beliefs that sounded familiar without being fully aware of them in daily life.

\section{Scoring}

Item-ratings are expressed in $\mathrm{mm}$, a higher score meaning stronger belief in the formulated statement (minimum score $=0$, maximum score $=100$ ). The summary score sheet enables to compute a dimensional belief score for every PD (12 subscales): all relevant item-scores are summed and then divided by 20 (minimum meanscore $=0$, maximum meanscore $=100$ ). In addition, the total PDBQ score can be calculated as an indication of overall maladaptive beliefs: mean belief 
scores for every PD are summed and then divided by 12 . Note that all PDBQ Scores are expressed on a dimension ranging from $0-100$. Cutoff norms for the pathological range are not available yet.

\section{Psychontetric Properties}

Large-scale studies on psychometric properties are ongoing. 'To date, only very preliminary data are available from three experimental studies in which subscales of the PDBQ were used to measure specific beliefs (Arntz, Dietzel, \& Dreessen, 1997; Dreessen, Arntz, Hendriks, Keune, \& van den Hout, 1998-b; Weertman, Dreessen, \& Arntz, 1998).

Arntz et al. (1997) administered six subscales of the PDBQ (items in randomized order) in a sample of 16 borderline PD patients, 12 Cluster-C PD patients without any borderline traits, and 15 normal controls. The internal consistency was found to be high for all six subscales (Cronbach's alpha Avoidant $=.96$; Dependent $=.92$; Obsessive compulsive $=.92 ;$ Paranoid $=.97 ;$ Histrionic $=.88 ;$ and Borderline $=$ 95). The stability of trait belief ratings was tested under rigorous conditions, by inducing a negative emotional state foregoing the second test of the PDBQ one week after the first test. Despite induction of negative emotions, the trait belief scores did not increase and test-retest correlations were high (total sample $r \mathrm{~s} \geq 88$; normals $r_{s} \geq .86$; Cluster-C PDS $r_{s} \geq .87$; borderline PD $r_{s} \geq .69$ ). The Arntz et al. (1997) study specifically focused on the borderline belief subscale of the PDBQ and proved the specificity of these beliefs for the DSM-III-R borderline PD (the PDBQ borderline scale discriminated most strongly the borderline PD patients from the Cluster-C PD patients and normal controls). Borderline and Cluster C PD diagnoses were established by using the Structured Clinical Interview for DSM-III-R personality disorders (SCID-II; for a description see First, Spitzer, Gibbon, \& Williams, 1995-a).

Dreessen et al. (1998-b; see also section 5.2 of the present thesis) administered the $P D B Q$ avoidant subscale in a sample of 57 undergraduate students who scored high or low on DSM-III-R avoidant personality pathology as assessed by the SCIDPersonality Questionnaire (SCID-mQ; for a description see First et al, 1995-a). As in the Arntzet al. (1997) study, the internal consistency of the PDBQ avoidant scale was excellent (Cronbach's alpha $=93$ ). Furthermore, the PDBQ avoidant score was significantly higher in the high SCID-HQ avoidant group than in the lOW SCID-HIQ avoidant group $[t(45.1)=4.68, p<.001]$, indicating that DSM-III-R avoidant personality pathology (SCID-IIQ) is indeed associated with the hypothesized characteristic beliefs, which constitute the PDBQ avoidant scale. Since other PDBQ scales were not included in the study, it was impossible to investigate the specificity of the Prop avoidant scale ${ }^{5}$

Weertman et al. (1998) administered the PDBQ and the SCID-MIQ dependent and paranoid subscale in a sample of 137 undergraduate students. Internal consistencies of the PDBQ dependent and paranoid scales were excellent (Cronbach's alpha $=$ $.88,92$, resp.). $\mathrm{PDBQ}$ dependent and paranoid scales also correlated significantly with the corresponding SCID-IIQ scales $(r=.62, p<.001 ; r=.63, \mathrm{p}<.001$ resp.). Compared to these correlations, correlations between the PDBQ scales and the noncorresponding SCID-IIQ were found to be lower (PDBQ dependent-SCID-IIQ paranoid $=.43$; $\mathrm{PDBQ}$ paranoid $-\mathrm{SCID-II}$ dependent $=.37$ ), which indicates specificity of the PDBQ scales. 
Finally, as hypothesized from cognitive theory, Dreessen et al. (1998-b) and Weertman et al. (1998) showed that all three investigated subscales of the PDBQ were associated with schema-congruent information processing biases: avoidant beliefs were associated with schema-congruent information processing biases on a pragmatic inference task; dependent and paranoid beliefs were associated with schema-congruent information processing biases on an adapted version of the Thematic Apperception Test. Overall, the SCID-IIQ was found to be a less sensitive predictor for disorder-congruent information processing biases than the PDBQ.

In conclusion, preliminary results on internal consistency, stability, specificity and validity of at least some of the $P D B Q$ subscales are promising. Larger-scale studies on psychometric properties of the PDBQ are needed.

\section{Discussion}

Two issues need to be discussed. First, the utility of the PDBQ may be limited by its length, particularly when it is administered in conjunction with other questionnaires. However, in an initial attempt to measure underlying Po beliefs we thought it would be best to generate many different belief formulations that are expected to cover the construct. It is difficult to decide beforehand which of the formulations will appeal the most to the respondents. In the future, a brief PDBQ may be developed.

Second, in general, measurement instruments for personality pathology, including PD belief questionnaires, are sensitive to state factors like anxiety and depression (cf. the acute psychiatric state) that might bias the report of information (Zimmerman, 1994). The content of the typical PD beliefs may resemble with the content of beliefs that are temporarily activated in Axis I disorders. For instance, the self-schema 'I am bad" will only be temporarily activated in a depressed person, who has an 'I am. OK' schema in a non-depressed state. However, people with personality disorders are assumed to have maladaptive self-schemas, such as I am bad", over the course of a lifetime, because they do not have an alternative, more adaptive schema available (Padesky, 1994). In other words, their self-schema refers to trait psychopathology and is pervasive, inflexible, stable and of long duration.

In an interview approach, the interviewer can be trained extensively to separate state beliefs from trait beliefs (e.g. PD beliefs). However, in self-report questionnaires, it is up to the respondent, who is a layman guided by written and / or oral instructions, to distinguish between state and trait beliefs. It remains to be demonstrated whether respondents are capable to do so in completing the PDBQ. In a first study on the impact of state factors on PDBQ report, Arntz et al. (1997) showed good stability of PDBQ beliefs of six subscales after induction of negative emotions.

In short, the current version of the PDBQ (Dreessen. Arntz, \& Weertman, 1996) attempts to define and quantify specific cognitive profiles of all DSM-IV PDS. It remains to be proven whether the attempt has succeeded or not. To date, only preliminary data on the $P D B Q$ are available, but they are encouraging enough to continue further research on psychometric properties. Cognitive measures for personality pathollogy, such as the $P D B Q$, are potentially promising tools for empirical research and clinical use. 


\section{Appendix 1}

\section{Item examples of the PDBQ (in Dutch)}

1. Regels zijn willekeurig en verstikkend

ik geloof dit helemaal niet

ik geloof dit helemaal

2. Anderen kunnen mijn gedachten lezen

ik geloof dit helemaal niet

ik geloof dit helemaal

3. Ik moeter alles aan doen om te voorkomen dat anderen iets op me aan kunnen merken

ik gelloof dit helemaal niet ik geloof dit helemaal

4. Ik ben niet zo goed opgewassen tegen moeilijke situaties als anderen

ik geloof dit helemaal niet

ik geloof dit helemaal

5. Het komt allemaal op mij neer om de dingen te doen die gedaan moeten worden

ik geloof dit helemaal niet ik geloof dit helemaal

6. Ik kan iets laten gebeuren door er gewoon aan te denken

ik geloof dit

helemaal niet

ik geloof dit helemaal

7. Ik ben in het leven onrechtwaardig behandeld en ik heb het recht om mijn verdiende deel op wat voor manier dan ook te verkrijgen

ik geloof dit

helemaal niet

ik geloof dit

hellemaal

8. Als mensen me echt leren kennen, zullen ze me afwijzen

ik geloof dit

helemaal niet

ik geloof dit

helemaal 


\section{notes}

1 Fydrich et al. (1996) translated the schema checklist (Beck et al, 1990, 359-363) in German language. Whereas the $\mathrm{BQ}$ has a 7 -point rating scale, they added a 5 -point rating scalle with each belief of the checklist, probably because they hadn't heard about the $\mathrm{BQ}$.

2 Recently, the SQ has been translated and adapted in Dutch (Sterk \& Rijkeboer, 1997).

3 Some of the beliefs of the list of Beck et al. (1990, pp. 359-363) were directly translated (with permission), some are reformulated [e.g. because we judged then to be unclear or incomplete. Examples are: 'I am needy and weak' was reformulated in 'I am weak and I need help from other people', 'I should be the center of attention' was reformulated in 'I should be the center of attention, otherwise I am nobody"], and some are not included in the PDBO [e.g. because to our opinion they referred more to facts (cq. symptoms) than to beliefs. Examples ane: "I cannot make decisions on my own' or 'I cannot tolerate unpleasant feelings']. At the time of the development of the PDBQ we did not know about the existence of the BQ (Beck, 1990).

4 To enhance variance among subjects we changed the categorical scoring system (yes/no) into $100 \mathrm{~mm}$ visual analogue scales.

5 The participants of this study completed all subscales of the SCID-MQ. However, we did not investigate discriminant validity, because the participants represented extreme groups, selected on their SCID-II avoidant score. 



\section{Avoidant personality disorder and implicit schema- congruent information processing bias: a pilot study with a pragmatic inference task ${ }^{1}$}

\section{Summary}

Cognitive theory of personality disorders hypothesizes that each personality disorder is characterized by typical maladaptive schemas and that these schemas direct the processing of information resulting in schema-congrttent biases. With regard to the avoidant personality disorder, these hypotheses were put to an initial test in a pilot study, using a self-report questionnaire to asses DSM-MI-R personality pathology, a belief questionnaire to assess avoidant schemas, and a pragmatic inference task to assess schema-congruent implicit attributional bias. Participants were nonclinical students $(n=57)$ who scored high or low on DSM-II-R avoiclant personality pathology. As predicted from cognitive theory, DSM-MI-R avoidant personality pathology was associated with avoidant beliefs $[t(45.1)=4.68, p<.001]$, and avoidant beliefs were associated with schema-congruent information processing bias $[1(55)=2.17$, $p=.02$, one-tailed test]. However, DSM-1I-R avoidant personality pathology was not associated with schema-congruent information processing bias [t(55) $=0.17, p=43$, one-tailed test 1 . Th addition to avoidant beliefs, low self-esteem was also related to the information processing bias. Social phobia and general personality pathology, two other control variables, were not. The findings warrant further study using the pragmatic inference task in a clinical group.

\section{Introduction}

The cognitive theory of personality disorders (e.g. Beck, Freeman, \& Associates, 1990) is developing into one of the main theoretical orientations in the personality disorder literature (Klein, 1993; Shea, 1993). It explains etiology and maintenance of personality disorders and it offers a treatment model. Moreover, it indicates specific problems to be expected in the treatment of Axis I disorders (e.g. fear of rejection by the therapist).

Cognitive theory of personality disorders is build on two main hypotheses. First, each personality disorder is hypothesized to be characterized by typical maladaptive cognitive schemas that deal with the self and the (social) world (e.g. Beck, et al., 1990). Second, these cognitive schemas are hypothesized to stimulate selective processing of information resulting in biased interpretations that maintain the pathology. In other words, the theory hypothesizes that patients with personality disorders will show schema-congruent information processing. To the 
best of our knowledge, there is no published research into this specific hypothesis. Other previous research has shown that self-schemas (e.g. dependency and assertiveness) in non-clinical participants were related to schema-congruent information processing biases (e.g. Bruch, Kaflowitz, \& Berger, 1988; Markus, 1977). Furthermore, a study by Martin, Ward and Clark (1983) showed that neuroticism, defined as a general enduring personality trait, was related to the favoring of negative information about the self, but not about others. From these results it can be expected that patients with personality disorders will show schema-congruent information processing biases. Regarding the disorder specific stimulus material, the existing information processing tasks would have to be adapted.

The present paper reports results from a study in nonclinical student participants that was designed as an initial test of the main hypotheses of the cognitive theory of personality disorders, the avoidant personality disorder (American Psychiatric Association, 1987) in particular. A self-report questionnaire was used to assess DSM-III-R avoidant personality pathology. A belief questionnaire was used as an explicit measure to assess the presence of cognitive schemas hypothesized to be rellated to the avoidant personality disorder. A pragmatic inference task ( $c$. Winters \& Neale, 1985) was used to assess an implicit attributional bias. The stimulus material of the pragmatic inference task was adapted to the avoidant personality disorder. Briefly, a pragmatic inference is a conclusion drawn by an individual, that consists of information that was not directly stated or logically implied by the original information (Harris \& Monaco, 1978). Indeed, Johnson, Bransford and Solomon (1973) have demonstrated that participants went beyond the information given and that they made tacit assumptions about information that was not stated (e.g. consequence of the action described). In cognitive theory, these inferred conclusions are hypothesized to be directed by cognitive schemas ( $c f$. the relevant knowledge a person already possesses). The pragmatic inference task was presented as a memory test, and participants were misled to believe that their answers gave us information about their general memory capacity and not about their personality characteristics. Therefore, the task is supposed to be rather insensitive to response bias (self-presentation in a positive way) and participants do not have to be aware of their schemas in order to show an effect on the task (Winters \& Neale, 1985).

In the pilot study we used a priming task for the purpose of activating avoidant schemas. Cognitive theory states that schematic processing may not occur unless schemas are activated (Beck et al., 1990; Safran, Segal, Hill, \& Whiffen, 1990). Furthermore, it is thought that the more valent schemas are, the more prominent their impact on information processing will be. Safran et al. (1990) have argued that, in the absence of a priming procedure, negative findings do not necessarily rule out the existence of schemas. In the present pilot study, the priming of schemas related to the avoidant personality disorder was done by a self-reference task. The main questions of the study were the following.

1 Is DSM-II-R avoidant personality pathology associated with a schema-congruent implicit attributional bias?

2 Are avoidant beliefs associated with a schema-congruent implicit attributional bias?

3 Is DSM-III-R avoidant personality pathology associated with avoidant beliefs? 


\section{Method}

\section{Participants}

Participants were selected from a larger sample of 192 first year college students of Health Sciences who responded to requests on notice boards to fill in a set of questionnaires. The set included an adapted and translated Dutch version of the SCID-Personality Questionnaire (SCID-MQ). Students were asked to participate in the present study if their SCID-IIQ avoidant personality score was either smaller in value than the first quartile or larger than the third quartile. 96 students met this criterion, of which 57 students participated. The remaining students either could not be reached or scheduled during the time of the experiment or refused to participate. The high SCID-IIQ avoidant group contained 29 participants. Their mean avoidant score was $705.7(S D=77.6, \min .=592, \max .=904)$. There were 28 participants in the low SCID-IIQ avoidant group with a mean avoidant score of $252.2(S D=56.0, \min .=119, \max .=332)$.

The mean age of the study sample was 21.3 years $(S D=4.2, \min .=18$, max. $=$ 41). $70 \%$ of the participants were women. There were no statistically significant differences between groups with regard to age $[t(35.3)=.86, p=.39]$ and sex $\left[\mathrm{chi}^{2}\right.$ $=2.35, p=.12]$.

The high SCID-IIQ avoidant group was not specifically selected being low on other SCID-IIQ scales. In fact, groups showed significant differences on most of the other SCID-IIQ subscales, in which the high SCID-IrQ avoidant group had higher pathology scores [avoidant, dependent, obsessive compulsive, passive aggressive, self-defeating, paranoid, schizotypal, histrionic, narcissistic, borderline: $4.1 \leq t s(55)$ $\leq 7.8, p s<.001]$. The group difference on the avoidant subscale was larger [t(55) = $24.5, p<.001$ ] than on the other SCID-liQ scales (compare $t$-values: $t=24.5$ vs. $4.1 \leq$ ts $\leq 7.8$ ). The groups showed no differences on the schizoid and antisocial SCID-IIQ subscales $[t(55)=0.23, p=.82, t(55)=-0.09, p=.92$, resp.]. Groups were also compared regarding self-esteem scores (Rosenberg Self-Esteem Scale) and social phobia scores (Social Phobia and Anxiety Inventory). As expected, there were significant group differences on both measures $[t(55)=-6.8, p<.001 ; t(55)=8.1, p<$ .001 resp.], indicating lower self-esteem and more social phobic complaints in the high avoidant SCID-IIQ group as compared to the low avoidant SCID-IIQ group.

\section{Materials}

SCID-PErsonality Questionnaire. An adapted Dutch version of the SCID-Personality Questionnaire (SCID-IIQ; see First, Spitzer, Gibbon, Williams, 1995-a: Dutch translation and adaptation by Arntz, 1987) was used for measuring DSM-III-R personality pathology, avoidant personality pathology in particular. This instrument is a self-report screening tool that corresponds to the interview questions in the Structured Clinical Interview for DSM-III-R Personality Disorders (SCID-II; Spitzer, Williams, Gibbon, \& First, 1990). Whereas the original SCID-1iQ has categorical scoring possibilities, the Dutch version we used contains $100 \mathrm{~mm}$ Visual Analogue Scales (VASs), because the questionnaire was originally translated for the purpose of measuring change with treatment. The avoidant subscale included 13 items with a possible score of 0 to 1300 in the Dutch version. High scores correspond with high avoidant personality pathology. 
In a nonclinical sample, Ouimette and Klein (1995) found an adequate testretest correlation for the dimensional score of the avoidant SCID-IIQ personality disorder $(r=0.69$; American version). They also found a fair median Kappa (0.56; American version) for the individual criteria of the avoidant personality disorder scale. To our knowledge, there are no other published psychometric data for the SCID-IIQ.

In the sample of 192 students, from which we selected the present study sample, the Cronbach's alpha for the avoidant subscale of the SCID-IIQ was 0.72. The present pilot study was conducted approximately six months after the SCID-IIQ screening in the large student sample. The test-retest reliability of the avoidant subscale was estimated by retaking this scale after the pragmatic inference task. The Intraclass Correlation Coefficient (ICC; Fleiss, 1986) of the SCID-IIQ avoidant score was 0.78, which indicates excellent test-retest reliability (Fleiss, 1981).

Personality Disorder Belief Questionnaire. The presence of avoidant cognitive schemas was assessed by administering the avoidant subscale of the Personality Disorder Belief Questionnaire (PDBQ2 ; Dreessen, Arntz \& Weertman, 1996). The avoidant subscale of the PDBQ addresses 20 beliefs that are hypothesized to be related to the avoidant personality disorder (e.g. "If others really get to know me, they will reject me'; I I act stupidly and not the way others expect me to do in social situations'). Instructions to the questionnaire request the participant to rate the strength of his/her belief in the formulated assumption on a $100 \mathrm{~mm}$ VAs $(0=$ 'I don't believe this at all', $100=$ 'T believe this completely'). To date, only preliminary data on psychometric properties of some of the PDBQ subscales are available. Results on internal consistency, stability, specificity and validity are promising (Arntz, Dietzel, \& Dreessen, 1997; Weertman, Dreessen, \& Arntz, 1998). Larger-scale studies are ongoing. The internal consistency of the PDBQ avoidant subscale in the present sample was very high (alpha $=.93$ ).

Other Questionnaires. The Rosenberg Self-Esteem-Scale (RSES; Rosenberg, 1965) and the Social Phobia and Anxiety Inventory (SPAI; Tumer, Stanley, Beidel \& Bond, 1989) were administered in the sample of 192 students for other purposes than the present study, but data were also used in the present study.

Priming Task. Priming of so-called avoidant schemas was done by a self-reference task (cf. Rogers, Kuiper \& Kirker, 1977). We presented 24 audiotaped adjectives relevant to avoidant schemas (plus 4 dummy adjectives at the start and at the end). Participants had to state whether the adjectives were self-descriptive or not. This self-reference task forces participants to use the self in the rating task and it enhances semantic involvement with schema-relevant information. This was supposed to activate avoidant schemas. Adjectives relevant to the avoidant selfschema can be schema-consistent (negative) or schema-inconsistent (positive) (Rojahn \& Pettigrew, 1992). Both types of adjectives were included in the priming task in random order (e.g. positive adjectives: self-confident, popular, entertaining, spontaneous; negative adjectives: boring, insecure, retiring, unsociable). Inclusion was based on consensus between the present authors. Indeed, high avoidant participants as compared to the low avoidant participants evaluated the positive schema-inconsistent adjectives less often as self-descriptive $[1(46.4)=-3.39, p<$ .001 , one tailed test] and the negative schema-consistent adjectives more often as self-descriptive $[\mathfrak{H}(55)=6.40, p<.001$, one tailed test $]$. 
The effect of the priming task was estimated by means of five VASs that measured momentary state conditions (dejection, nervousness, self-assurance, selfsatisfaction and self-confidence) (e.g. 'How dejected do you feel right at this moment?'). Scores on these VASs were supposed to reflect the level of activation of the avoidant schemas. This indirect measure was preferred above direct questioning of the avoidant schemas in order to maximize the intransparency of the study.

Pragmatic Inference Task. Following Winters \& Neale (1985) we constructed a pragmatic inference task consisting of 15 audiotaped short stories highly ambiguous as to the cause of the event mentioned in the story. Two causes were vaguely hinted at, without being explicitly or logically linked to the event. One cause was related to avoidant schemas and the other was not. For example, one story described a situation in which the participant imagines entering a room full of birthday guests and nobody says hello. Two causes were implied:

1 The guests prefer to talk to the others and not to you, or

2 The guests did not see you coming in because it was crowded.

The event and the suggested cause related to avoidant schemas for all 15 stories are presented in Appendix 1.

Each time, after listening to three short stories, participants were instructed to respond on paper to four multiple-choice questions about each story. In the target question, participants were asked to remember which cause contributed to the event mentioned in the story. In another question, they were asked for memory of implied information not related to causality, and in two other questions they were asked for memory of stated facts. Participants were asked to respond after three stories instead after each story because the distinction between given and inferred information becomes less clear at later recall (Winters \& Neale, 1985). We wanted to prevent participants to see through the task that was presented as a memory task of stated facts.

The inference task was validated by six experienced clinicians (including the second author) who, for other research purposes, were trained extensively in diagnosing personality disorders with the Structured Clinical Interview for DSMIII-R personality disorders (SCID-II; Spitzer et al., 1990). They were asked to read 20 stories and to endorse whether the two possible answers to the target questions characterized the avoidant personality disorder or not. Stories were excluded from the inference task if two or more clinicians judged the hypothesized avoidant answer not to be characteristic of the avoidant personality disorder or the hypothesized non-avoidant answer to be characteristic of the avoidant personality disorder. Consequently, one story was dropped. Next, pillot work was conducted on a group of 20 college students to ensure variability among the answers to the target question. In order to be included in the inference task, one of the two suggested causes corresponding to each target question had to be endorsed by at least 5 out of 20 students. 11 out of 19 stories met this criterion. The other 8 stories were modified and repiloted with a new group of 20 college students and 4 more stories met the criterion. Eventually, 15 stories were included in the inference task. An example of one story and its questions is given in Appendix 2.

The pragmatic inference task ended with several evaluative questions concerning this task (see Results section). 


\section{Procedure}

The study was executed in a soundproof room. The stimuli of the priming task and the inference task were presented to the participants via headphones. All participants followed the same procedure. After obtaining informed consent, we first administered the priming task and then the pragmatic inference task. All instructions were read aloud from a sheet of paper. In the instructions for the priming task, participants were asked to indicate whether the word, presented via headphones, was self-descriptive or not in about 5 seconds. An example was given and it was stressed that they should always make a choice between 'yes' or 'no'. Participants were asked to give honest answers for the sake of the study and anonymity was explained and guaranteed again. After these instructions, the participant was left alone in the room and we started the audiotape in an adjacent room.

The inference task was presented as a memory test. Participants were told that they would hear a number of short stories that were audiotaped. They were asked to listen very carefully and to memorize as much information as possible. It was predicted that it would sometimes be difficult to maintain their concentration on the stories and, therefore, some questions might be difficult to answer afterwards. Participants were instructed to answer every question. If they could not remember the issue properly, their best guess should be based on the story and not on what is generally to be expected under the described circumstances in the story. Finally, we instructed them to visualize the story, because it would be helpful for remembering detailed information. We hoped it would lead them to identify themselves with the principal character ('you'). We chose not to give explicit instructions to identify with the principal character in order to minimize the transparency of the task. After these instructions, the participant was left alone in the room and we started the audiotape in an adjacent room.

The five VASs measuring momentary state conditions were filled out before and after the priming task and again after the pragmatic inference task. Finally, the participants completed the avoidant subscale of the PDBQ and the SCID-III and the evaluative questions concerning the pragmatic inference task. At the end, participants were debriefed and received a small amount of financial compensation for their participation in the study.

\section{Results}

\section{Schema Actiontion Check}

Table 1 presents the means and standard deviations of the ratings on the five VASs (c). 'priming-effect' variables). Before the ratings of the five vass were submitted to a multivariate analysis of variance, they were rescaled, so that high scores on all VAss refer to a high priming effect (that is, feeling dejected, nervous and having a low sense of self-assurance, self-satisfaction and self-confidence).

A 2 (Group) $\times 3$ (Time) multivariate analysis of variance (Wilks) on ratings of the five VASs revealed a significant main effect for Group $[F(5,51)=3.1, p=.001]$ and Time $[F(10,212)=7.1, p<.001]$. No Group $\times$ Time interaction effect was found $[F(10,212)=0.7, p=.76]$. The Group-effect indicates that the high SCID-IIQ avoidant group as compared to the low SCID-IIQ avoidant group scored higher on the 
'priming-effect' variables throughout the entire experimental study. The Timeeffect indicates that, overall, ratings on the 'priming-effect' variables decreased as the study progressed. The non-significant Group x Time interaction indicates that neither the priming task nor the pragmatic inference task further increased feelings of dejection or nervousness or decreased the sense of self-assurance, selfsatisfaction and self-confidence in the high SCID-MQ avoidant group. However, it cannot be concluded that the priming task was superfluous, because it may have successfully caused the groups to keep up the between-group differences on the VAS tests from the start throughout the entire experimental study.

TABLE 1

Means and standard deviations of the VAS scores on self-esteem, dejection and nervousness preceding and following the priming task (pre-posttest) and following the pragmatic inference task (follow-up)

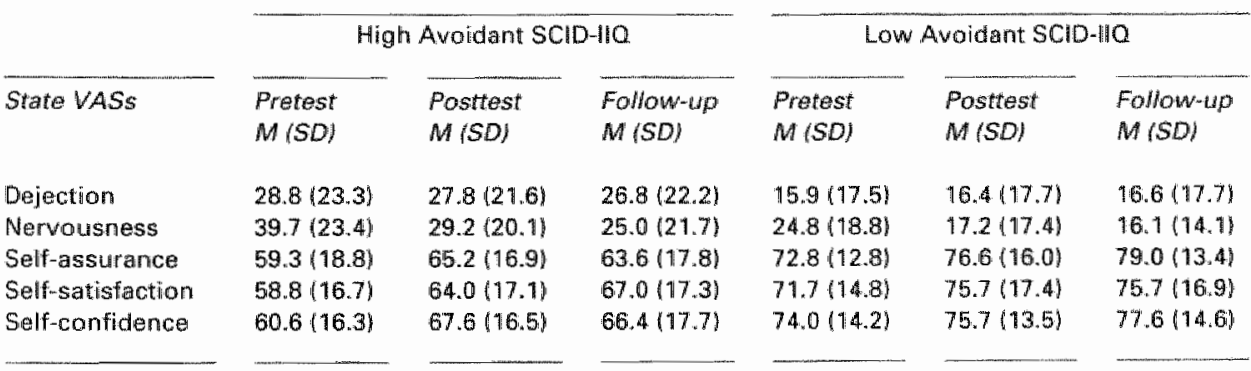

SCID- $\| Q=S C I D \times$ Personality Questionnaire

$M=$ mean

$S D=$ standard deviation

\section{Pragmatic Inference Task - General Findings}

With regard to stated facts (2 questions with each story), the mean percentage recall was extremely high for both groups [high vs. low avoidant SCID-IIQ: $M=$ $95 \%, S D=4 \%$ vs. $M=92 \%, S D=5 \%$ ]. The percentage recall of stated facts was significantly higher in the high SCID-IIQ avoidant group compared to the low SCIDUQ avoidant group $[t(55)=2.33, p=0.02]$.

As far as the causal inference questions were concerned, the probability, regardless of group, of endorsing either an avoidant or a non-avoidant inference was adequate: the mean percentage of avoidant attributions was $47 \%(S D=19 \%)$ and the mean percentage of non-avoidant attributions was $53 \%(S D=19 \%)$.

The evaluative questions ( $100 \mathrm{~mm}$ vass) concerning the pragmatic inference task yielded the following results: participants indicated that they identified themselves with the principal character of the stories ('you') to a high degree ( $M=$ $69, S D=20$ ). The mean value regarding participants' certainty about the given answers was $64(S D=16)$. We checked to what extent participants attributed uncertainty about answers to an external cause (information not given) or an internal cause (information given but forgotten). The mean value for 'information not given' was $69(S D=25)$. The mean value for 'information given but forgotten' was $37(S D=22)$. These results indicate that participants tend to believe more often that information was not given than that information was forgotten. However, 
the final question showed that for only 5 or less of the 60 questions the majority of participants $(63 \%)$ were $100 \%$ sure that the information was not given in the story. Note that in 30 questions they were asked for memory of implied information. The above results indicate that most of the times participants are more or less in doubt about whether or not the information was given. In other words, we were reasonably successful in misleading the participants to believe that all the answers referred to stated facts. There were no between-group differences for the evaluative questions ( $|t s(55)| \leq 1.43$, ps $\geq .16)$.

\section{Pragmatic Inference Task - Implicit Attributional Bias}

High us, Low SCID-IYQ Avoidant Group. As is shown in Table 2, the number of avoidant attributions was not significantly different between groups $[t(55)=.17$, $p=.43$, one tailed test $]$, indicating that high SCID-IYQ avoidant participants as compared to low SCID-IIQ avoidant participants did not show a schema-congruent implicit attributional bias.

High ws. Low PDBQ Awoidant Group. Participants were regrouped according to their PDBQ avoidant score instead of by their SCID-IIQ avoidant score. 13 participants changed group (high avoidant SCID-IIQ/ low avoidant PDBQ: $n=7$; low avoidant SCID-IIQ/ high avoidant PDBQ: $n=6$ ). The mean PDBQ avoidant score in the low PDBQ avoidant group was $261.6(S D=75.1, \min .=70, \max .=423)$. The mean PDBQ avoidant score in the high PDBQ avoidant group was $706.1(S D=245.5, \mathrm{~min} .=429$, max. $=1313$ ). The mean number of avoidant attributions and standard deviations for both PDBQ groups are shown in Table 2. High PDBQ avoidant participants made significantly more avoidant attributions than low PDBQ avoidant participants [ $t(55)$ $=2.17, p=.02$, one-tailed test). In other words, participants with a high score on the PDBQ avoidant subscale showed an implicit schema-congruent information processing bias compared to participants who scored low on the PDBQ avoidant subscale.

TABLE 2

Means and standard deviations on the pragmatic inference task for the SCID-\|IO and the PDBO

Group

\begin{tabular}{|c|c|c|c|}
\hline \multicolumn{2}{|c|}{ High Awoidant } & \multicolumn{2}{|c|}{ Low Avoidant } \\
\hline$M$ & $S D$ & $M$ & SO \\
\hline 6.4 & 27 & 6.3 & 3.0 \\
\hline 7.2 & 2.5 & 5.6 & 2.9 \\
\hline
\end{tabular}

SCHD $140=$ SCD-Personality Questionnaite

$\mathrm{PDBO}=$ Personality Disorder Balief Questionnaire 
DSM-IT-R Avoidant Pathology and Ratings on Avoidant Beliefs

The high SCID rle avoidant group showed sigmificantly higher ratings on the avoidant beliefs than the low SCID-IIQ avoidant group $[t(45.1)=4.68, p<.001$, one tailed test].

\section{Post-Hoc Analyses}

In order to get an impression of the specificity of the association found between the avoidant schemas and the bias, post-hoc analyses were done. We evaluated the associations between pragmatic inference scores and some relevant and available control variables:

1 the scores on the 11 Scm-Iro subscales other than the avoidant subscale,

2 a self-esteem score, and

3 a social phobia score.

A factor analyses on the 11 ScID-mQ subscale scores revealed one clear factor solution, accounting for $56 \%$ of the variance, 9 subscales loading on it. The scores of those 9 subscales were added up, resulting in general sciD-IIQ sumscore. Schizoid and antisocial scores did not load on this general factor and were excluded from the SCID-no sumscone. They were analyzed separately.

Tutests (high vs. low on one of the control variables) revealed that the SCID-IIQ sumscore and the social phobia score were not associated with the information processing bias [SCID-IIQ sumscore: $t(55)=-.74, p=.47$; social phobia score: $t(55)=$ $77, p=.44]$. Low self-esteem, on the other hand, was found to be associated with the information processing bias $[l(55)=-2.65, y=.01]$. Participants with low selfesteem made more avoidant attributions than participants with high self-esteem. The schizoid score was also associated with the implicit information processing bias, but in the opposite direction. Participants with a high schizoid score made less avoidant attributions than participants with a low schizoid score $[t(55)=$ $-2.23, p=.03]$. The antisocial score was not associated with an implicit information processing bias $[t(53)=-1.16, p=.25$; note that there were missing data on the scrome antisocial subscale in two cases].

Next, a multiple regression analysis was carried out incorporating all grouping variables thigh/low: SCID-rQ avoidant, PDBQ avoidant, sCID-HQ sumscore, social phobia, self-esteem, SCD-mQ schizoid and SCID-HQ antisocial]. This analysis assessed whether the observed PDBQ avoidant effect, the self-esteem effect and the SCD-11Q schizoid effect could be "explained" by some inter-relationships between the conceming grouping variable and the other grouping variables. High PDBQ avoidant score and low self-esteem were still significantly associated with the information processing bias $[\mathbb{B}=0.27, t=1.72, p=.045$, one-tailed; $\mathbb{B}=-0.40, t=$ $-2.4, p=.02]$, indicating that the $P D B Q$ avoidant effect is not accounted for by selfesteem or any of the other grouping variables. Similarly, the self-esteem effect is not accounted for by PDBQ avoidant psychopathology or any of the other grouping variables. The schizoid effect disappeared after controlling for the other grouping variables. 


\section{Discussion}

To the best of our knowledge, this is the first study that evaluated responses to an information processing task in personality disorders. Information processing tasks provide an interesting methodology for validating cognitive theory of personality disorders. DSM-III-R avoidant personality pathology was assessed by a self-report questionnaire, the SCD-IIQ. Cognitive avoidant schemas were assessed by a belief questionnaire, the $\mathrm{PDBQ}$.

As predicted by cognitive theory, DSM-III-R avoidant personality pathology was associated with PDBQ avoidant beliefs, and PDBQ avoidant beliefs were associated with a schema-congruent implicit attributional bias. These are important first findings in validating cognitive theory of personality disorders. Schema-congruent information processing biases are a crucial element in cognitive theory of personality disorders, first because they are bellieved to underlie emotional and behavioral problems, and second because they confirm the existent schemas and thereby maintain personality pathology.

The next step would be to demonstrate the specificity of the above findings. However, being only a pilot study, we did not administer other PDBQ scales and therefore we cannot investigate the specificity of the association between the PDBQand SCID-IIQ-avoidant scale. Also, we did not employ control groups, such as another DSM personality disorder group or another PDBQ personality disorder group and therefore no final conclusions can be drawn on the specificity of the bias for avoidant $P D B Q$ personality pathology. Some conclusions about specificity are suggested by the post-hoc analyses.

The post-hoc analyses revealed that SCID-nQ personality pathology excluding avoidant and schizoid pathology was not associated with the bias. The same held for social phobia pathology, as measured by the SPAI. These findings hint at a specific relationship between avoidant schemas and the schema-congruent information processing bias. In addition to avoidant beliefs, low self-esteem, as measured by the RSES, was found to be associated with the bias on the pragmatic inference task. This effect by itself is not surprising, since avoidant personality pathology is characterized by low self-esteem. Moreover, the pragmatic inference task assesses implicit internal attribution processes after failure in social interactions which can be expected to relate to low self-esteem in general, regardless of high or low avoidant personality pathology. In other words, it can be hypothesized that all psychopathology which is characterized by explicit or implicit low self-esteem in social interactions will show an information processing bias on our pragmatic inference task. Indeed, a multiple regression analysis showed that the low selfesteem effect could not be explained by variation in the other grouping variables, such as PDBO avoidant personality pathology, indicating a specific relationship between low self-esteem and the bias on our task. Interestingly, the PDBQ avoidant effect could not be explained by variation in the other grouping variables also, indicating a specific relationship between POBQ avoidant personality pathology and the bias on our task.

Schizoid personality pathology was also associated with an implicit information processing bias, but in the opposite direction of the PDBQ avoidant and the low self-esteem effect. Participants with a high schizoid score made less avoidant attributions than participants with a low schizoid score, which is in line with the 
detachment from social relationships and the restricted range of expression of emotions in interpersonal settings that is characteristic of schizoid personalities (APA, 1994). However, this effect disappeared after controlling for interrelationships with the other grouping variables.

In contrast to one of our main hypotheses, DSM-III-R avoidant personality pathology was not associated with schema-congruent information processing bias. Explanations to be considered reflect methodological weaknesses of the study and / or theoretical issues. First, we used a sub-clinical group of participants. Possibly, the biases occur only in a clinical group with full-blown personality disorders.

Second and in a comparable vein, the divergence between groups might not have been large enough, even though we were able to select quite high and low avoidant participants.

Third, for the assessment of DSM-IIM-R personality pathology we used a selfreport questionnaire of which no validity data were available. In general, selfreport personality disorder questionnaires are believed to be less valid instruments than (semi)structured interviews (Dreessen \& Arntz, 1998-a; Zimmerman, 1994). Indeed, the high SCID-IIQ avoidant group was characterized by high scores on most of the other SCID-IIQ scales, suggesting that the SCID-mi is more a measure for general personality pathology, and to a lesser degree a measure for specific personality pathology.

Fourth, it could be argued that the avoidant schemas were present, but not properly activated in the high DSM-III-R avoidant group, which led to the nonsignificant results. Results on the 'priming-effect' variables suggest that maladaptive schemas were active in the high avoidant group. However, we cannot be certain whether these were avoidant schemas. The vAS scores may also reflect the effects of mood or the effects of other maladaptive schemas that were activated in the high DSM-III-R avoidant group. On the other hand, we did find a relationship between avoidant belief ratings and the bias, which makes this fourth explanation less plausible.

Fifth, theoretically, cognitive schemas have a more direct relationship with information processing biases than DSM-III-R personality pathology, which might explain that only the PDBQ-avoidant score, and not the SCID-IIQ avoidant score was associated with avoidant inferences in the present subclinical group of participants. Note that a more direct theoretical relationship between cognitive schemas and information processing biases does not imply a tautological relationship. The belief questionnaire and the pragmatic inference task measure different aspects. The former is an explicit measure of the presence of cognitive schemas. The latter is an implicit measure of information processing (which is hypothesized to be directed by cognitive schemas).

In conclusion, the present study reported on the first steps in a relatively unexplored area. As predicted from cognitive theory of personality disorders, avoidant personality disorder beliefs were associated with an implicit schemacongruent information processing bias and with DSM-HII- $R$ avoidant personality pathology. These are important first findings in validating cognitive theory of personality disorders. Further study into cognitive theory of personality disorders using reliable and validated diagnostic instruments for personality disorders in well controlled experiments is needed. 


\section{notes}

1 Submitted for publication as: Dreessen, L., Amtz, A., Hendriks, T., Keune, N., \& Van den Hout, M. A. (1998-b). Avoidant personality disorder and implicit schema-congruent information processing bias: a pilot study with a pragmatic inference task.

2 The POBQ has recently been developed in our institute and consists of 12 subscales, corresponding with the DSM-IV personality disorders (APA, 1994). Each subscale contains 20 beliets that characterize one particular personality disorder. The beliets are partly formulated on the basis of Beck ef al. (1990; Pp. 359-363, with permission) and partly hypothesized by the constructors, Currently, psychometric properties are being investigated. 


\section{Appendix 1}

\section{The event and the suggested cause related to avoidant schemas for all 15 stories of the pragmatic inference task}

1. You enter a room full of birthday guests and nobody says hello (the guests prefer to talk to the others and not to you).

2. Your boss does not respond to the remark you make in the meeting (your boss thinks you are incompetent and you irritate him).

3. Your date, who you met for the first time last month, does not show up the second time you arranged to meet (your date didn't want to go out with you very much).

4. You have been trying hard to find a new job at a law center, but all to no avail (you don't make the right impression).

5. The person who you are out with for the first time is not very enthusiastic at parting (the person was disappointed about your lack of conversation).

6. In the end you decide not to go for the planned walk in the mountains (you were dreading it).

7. You are not present at the party where you were supposed to give a speech (you are in too much doubt about the quality of your speech).

8. Your colleague gives you a compliment about your appearance (your colleague does not like you, but is currying favor).

9. You are very quiet and aloof in the company of some people you don't know (you are afraid to give your opinion on the subject).

10. Your date, with whom you want to start a serious relationship, does not show up (your date is not interested in a serious relationship with you).

11. You don't want to go to the farewell party of your former employer (unexpectedly, you have to go all by yourself).

12. You decided not to go to work today (you do not dare look your boss in the face).

13. A group of people do not approve of the plan you presented (your presentation was not very convincing and did not radiate much self-confidence).

14. You intended to criticize your colleague on being late at work regularly, but you still didn't do it (you are afraid to tell your colleague).

15. It was your turn, but the baker served someone else instead of you (the baker found it much more important to serve the other person instead of you). 
Example of a story of the pragmatic inference task and its corresponding questions

'You arrive at a birthday party. On entering the room nobody says hello to you. You get the impression that some people did indeed see you enter the room, but everybody continues to talk to each other. For a moment you are not sure where to go. You remember a big party one month ago. It was a crowded party and nobody noticed people coming in. People were walking around all the time joining small groups of people that were busy talking to each other. The present party is quite different from the party last month. Now there are chairs and some nice music is being played. You feel like a beer'.

1. What was being celebrated? (factual)

A a marriage

B a birthday

2. Who was present at the party? (noncausal inference)

A people who you did not know for the most part

B people who you knew for the most part

3. Why does nobody says hello when you enter the room? (causal inference)

A. They prefer to continue talking to the others

B It is already very crowded, so nobody notices a new guest entering the room

4. What do you do the moment you enter the room? (factual)

A You walk to the bar

B You join a group of people 


\section{General discussion: implications from and for research}

Since results of the separate studies have been reported and discussed in detail in the previous chapters, only main issues will be reviewed in the current chapter.

\section{Assessment of personality disorders by the Structured Clinical Interview for DSM-III-R Personality Disorders}

\section{Reliability}

The development of standardized instruments for the assessment of personality disorders (PDs) has greatly improved diagnostic reliability. As pointed out in the general introduction, (semi)structured interviews are to be preferred above selfreport questionnaires and unstructured clinical interviews. One of the most popular and frequently used semistructured interviews is the Structured Clinical Interview for DSM-IM-R Personality Disorders (SCD-II; Spitzer, Williams, Gibbon, \& First, 1990). Regarding interrater reliability, the joint-interview approach is used most frequently. For the SCID-III, this approach has yielded adequate-to-good interrater reliabilities (see review by Zimmerman, 1994, p. 226). However, two important issues are not taken into account using this approach: rater variance in the elicitation of information and patient variance in providing information at two different times. In fact, rater variance in the elicitation of information has been suggested to be a major limitation of interviews (Zimmerman, 1994). Extensive training can decrease variation in the additional (unstructured) probe questions, which are needed to clarify answers in semistructured interviews. However, the impact of age, sex, and personality of the interviewer in interaction with such demographic variables of the patient are much less under control. Short-interval test-retest interrater reliability studies take into account these sources of variation, and therefore it is of crucial importance to study whether semistructured interviews pass this test.

In three prior studies using the SCID-II, short-interval test-retest reliabilities ranged from poor to good (First et al., 1995-b; Hennch, Lauer, \& Fydrich, 1994; Malow, West, Williams \& Sutker, 1989). Our study, described in section 2.1, is the first that evaluated this kind of reliability in a group of outpatients and also the first that evaluated single SCID-II traits and dimensional scores. In contrast to the large study of First et al. (1995-b), our interviewers received extensive training and were instructed to address all questions of the interview (no pre-interview questionnaire was used, whereupon only positive items were checked in the interview). Overall, but with the exception of the histrionic traits, the short-interval test-retest interrater reliability of the SCID-II was found to be satisfactory in our group of outpatients whose main complaint was mostly an anxiety disorder.

These results are promising for the use of the SCID-II as an assessment instrument in clinical and research practices in outpatient samples. However more of this research is needed. First, to replicate our findings. Replication is needed. 
because of the small sample size and the low illness base rate of some PD variables. With small sample sizes reliability coefficients are less stable. With low base rates a few disagreements can have a marked impact on the overall reliability (Note that $P D$ variables with very low base rates were already excluded from the analyses). Moreover, rater characteristics and sample characteristics may have a considerable impact on reliability in a particular study. Therefore replication is required, before generalizing the results to other raters and other outpatients.

Second, more research is needed to evaluate the PD variables, that could not be analyzed in our sample (such as most of the categorical full-blown PDs) because they did not meet the illness base rate requirements.

Third, recall that we used the SCID-II interview for DSM-III-R PDS. Further studies should establish test-retest reliabilities using the SCID-II for DSM-IV PDS (First, Spitzer, Gibbon, Williams, \& Benjamin, 1994), which has recently been translated into Dutch language (Weertman, Arntz, \& Kerkhofs, 1997).

Note that it is important to study reliability in outpatient samples specifically, because these samples are more likely to contain less prototypic and less severe PD cases, of whom the PD diagnoses are more difficult to reproduce than in clear cut cases (e.g. shown by Vandiver \& Sher, 1991; Skodol, Oldham, Rosnick, Kellman, \& Hyler, 1991).

\section{Validity}

Apart from being reliable, a diagnostic instrument should also be valid, that is, measuring the construct that it is suppose to measure (Cronbach \& Meehl, 1995). This is a complicated matter in pDs. It is unknown whether relatively brief interviews of a couple of hours are capable of validly evaluating long-standing pervasive problems. Unfortunately, there is no yard-stick against which to compare the diagnoses. As explained in the general introduction and in section 2.2, one of the validity questions in the assessment of PDS, concerns the validity of patient reports. Several authors have argued that reports of close informants are an important supplement to patients reports (e.g. Stangl, Pfohl, Zimmerman, Bowers, \& Corenthal, 1985; Bernstein et al., 1997). The difficulty is how to decide what source of information is more valid when the patient's and informant's answers conflict. It can be postulated that patient reports may be more valid for some PDs, whereas informant reports may be more valid for others (e.g. avoidant and dependent PDS vs. narcissistic and antisocial PDS). However, empirical evidence for favoring one source of information over the other is lacking. Several studies (Bernstein et al., 1997; Dowson, 1992; Riso, Klein, Anderson, Ouimette, \& Lizardi, 1994; Steketee, 1990; Tyrer, Alexander, Cicchetti, Cohen, \& Remington, 1979; Zimmerman, Pfohl, Coryell, Stangl, \& Corenthal, 1988) have demonstrated poorto-modest concordance between patient and informant based diagnoses, indicating that at least one of the two sources is invalid with respect to the individual criteria. Our study, described in section 2.2, showed also poor-to-modest patient-informant concordance using the SCID-II. In our study we attempted to determine which of the two sources of information is the most valid. Comparisons with therapist based diagnoses suggested that patient reports are more valid than informant reports, but it could not be excluded that both patient and therapist reports were invalid. Interestingly, we found that patient-informant couples with good- 
quality relationships showed higher (but not spectacularly) concordance rates than couples with less good-quality relationships. This increase in convergent validity supports the use of patient reports in at least a subgroup of patients, evaluating particular PDs. This preliminary conclusion cannot be generalized to the subgroup of patients with less good-quality relationships, for we do not know whether such patients report on their personality functioning similarly to patients who have good-quality relationships (e.g. less good-quality relationships may be associated with highly socially desirable answers by patients).

In conclusion, our study did not show convincing evidence for or against the use of patient reports in diagnosing PDs with the SCID-II. Clearly, more research is needed. For instance, it would be very exciting if it turned out that informant based $P D$ diagnoses have more predictive value in treatment responsivity to Axis I therapies than patient based PD diagnoses. Also, it would be interesting to use a more strict requirement in patient interviews by proposing that inflexible, maladaptive, and interfering traits (referring to cognition, affectivity and behavior) have to be noticed by 'healthy' bystanders (e.g. family members, friends, colleagues), or otherwise they are not abnormal. Such a definition would, of course, have some implications for the formulation of criteria in the DSM.

In the field of PDS, we are not only struggling with the validity of PD measurement instruments, but also with the validity of PD constructs and diagnostic criteria (Shea, 1992; Zimmerman, 1994). In other words, do PDs exist (validity of the theoretical construct)? What diagnostic criteria quantify the construct (validity of the diagnostic criteria)? And finally, do the assessment instruments measure what they are supposed to measure (validity of the measurement instrument)? For example, several authors pointed out that it remains to be demonstrated that the diagnostic criteria proposed in the DSM-HI-R and DSMIV are valid themselves (e.g. Zimmerman, 1994, Widiger, 1992). The diagnostic criteria refer to cutoff points of PDs and to the traits considered to be specific for a PD category. The thresholds for most of the PD diagnoses (e.g. at least 4 of the 7 paranoid traits) were based simply on the expert consensus of the Personality Disorder Advisory Committee. No data guided the decisions for placing the cutoff points (Widiger, 1992). The result is that patients without for instance a dependent $\mathrm{PD}$, may have a lot (but not enough) dependent characteristics causing significant functional impairment or subjective distress. Also, regarding the traits, it is not sure whether they cover the essence of the PD construct. In addition, the polythetic format of the DSM-III-R and DSM-IV may cause heterogeneity within the PD categories ${ }^{1}$ (the same holds for several Axis 1 disorders like major depressive disorder and panic disorder). Because both the PD instruments and the DSM diagnostic criteria are not properly validated yet, studies finding that PDs are not associated with some other feature are difficult to interpret. For instance, we demonstrated that full-blown PDs, assessed by the SCID-II, did not influence the results of cognitive and behavioral therapies for anxiety disorders (section 3.1 and 3.2). Does this indicate that the instrument failed to measure validly the diagnostic criteria? Or does this indicate that the diagnostic criteria for 130 are invalid or that the PD entity does not exist? $\mathrm{Or}$, does this indicate that the PDS, being a valid construct with valid diagnostic criteria that can be validly measured, do not influence these therapies? No definitive answer can be given yet. For the 
moment, it can only be concluded from our studies that full-blown PDS, as measured with the SCID-II relying on patient reports, were found to be unrelated to treatment outcome in cognitive and behavioral therapies for panic disorder and obsessive compulsive disorder (and for a group of various anxiety disorders lumped together). The validity of PD constructs, PD criteria and PD instruments are important issues for further study.

\section{Impact of personality patholog $y^{2}$ on treatment of anxiety disorders}

\section{General Findings}

In studying the impact of personality pathology (PP, e.g. personality disorders) on treatment of Axis I anxiety disorders it is important to distinguish between impact on the process of therapy and impact on the outcome of the therapy. The latter can be expressed in terms of Axis I end-state functioning or in terms of amount of improvement compared to the pretest. Axis I patients with PP can be expected to have more severe acute complaints at pretest than Axis I patients without PP (e.g. Study 2, section 3.1). Therefore, even if their decrease in Axis I symptoms would be equal to patients without PP, their Axis I end-state functioning would be still worse. Figure 1, visualizes several pathways for PP to affect treatment outcome via treatment process. For the sake of simplicity, the variables 'personality pathology (PP)', 'complications' and 'improvement' are dichotomized in the model, whereas the author prefers to see them as dimensional variables. First, PP may' or may not cause complications in the Axis I therapy process. Second, complications may or may not be solved during the Axis I therapy. Third, no complications or solved complications will result in equal Axis I improvement, or even equal Axis I end-state functioning of patients with and without personality pathology in the short term, but not necessarily in the long term (In the long term, Pp may have a negative impact on relapse rate). Unsolved complications will result in less Axis I improvement of patients with PP compared to those without PP in short term, but not necessarilly in the long term. In the long term, these patients with PP may reach equal Axis I improvement compared to the pretest, or even equal Axis I end-state functioning (with or without additional therapy). Type and severity of PP, as well as the assessment that is used to estimate PP, are important variables to take into account in studying the impact of $\mathrm{PP}$ on the Axis I therapy. Moreover, type and severity of Axis I disorder and type of Axis I treatment technique may also interact with the presence of concomitant PP.

In the three studies, described in section 3.1 and 3.2, it was found that categorical, patient-derived SCID-II PD diagnoses did not affect the amount of improvement of cognitive behavioral therapies (CBT) for anxiety disorders in short term. General as well as several specific PD variables were studied. Since categorical PD diagnoses are criticized for having arbitrary thresholds, it is of importance to evaluate the effect of dimensional PD variables as well. Interestingly, the evaluation of dimensional PP variables showed similar results, except for obsessive compulsive traits. These traits were related to less Axis I improvement in patients treated for panic disorder and in patients treated for all kinds of anxiety disorders [ $c$. the old notion that rigidity has a negative influence on therapy outcome]. Another im- 
portant finding concerns the drop-outs. In all three studies, study completers and drop-outs did not differ with regard to personality pathology suggesting that patients with PDs do not tend to select themselves out of Axis I therapies for anxiety disorders, once they have started. Moreover, in the obsessive compulsive disorder study, change scores of drop-outs were estimated (considerable improvement vs. no improvement) and then combined with data of completers. Still, pp did not affect CBT outcome negatively. These findings do not rule out the possibility that PDs prevent people from getting into therapy for their Axis I disorders.

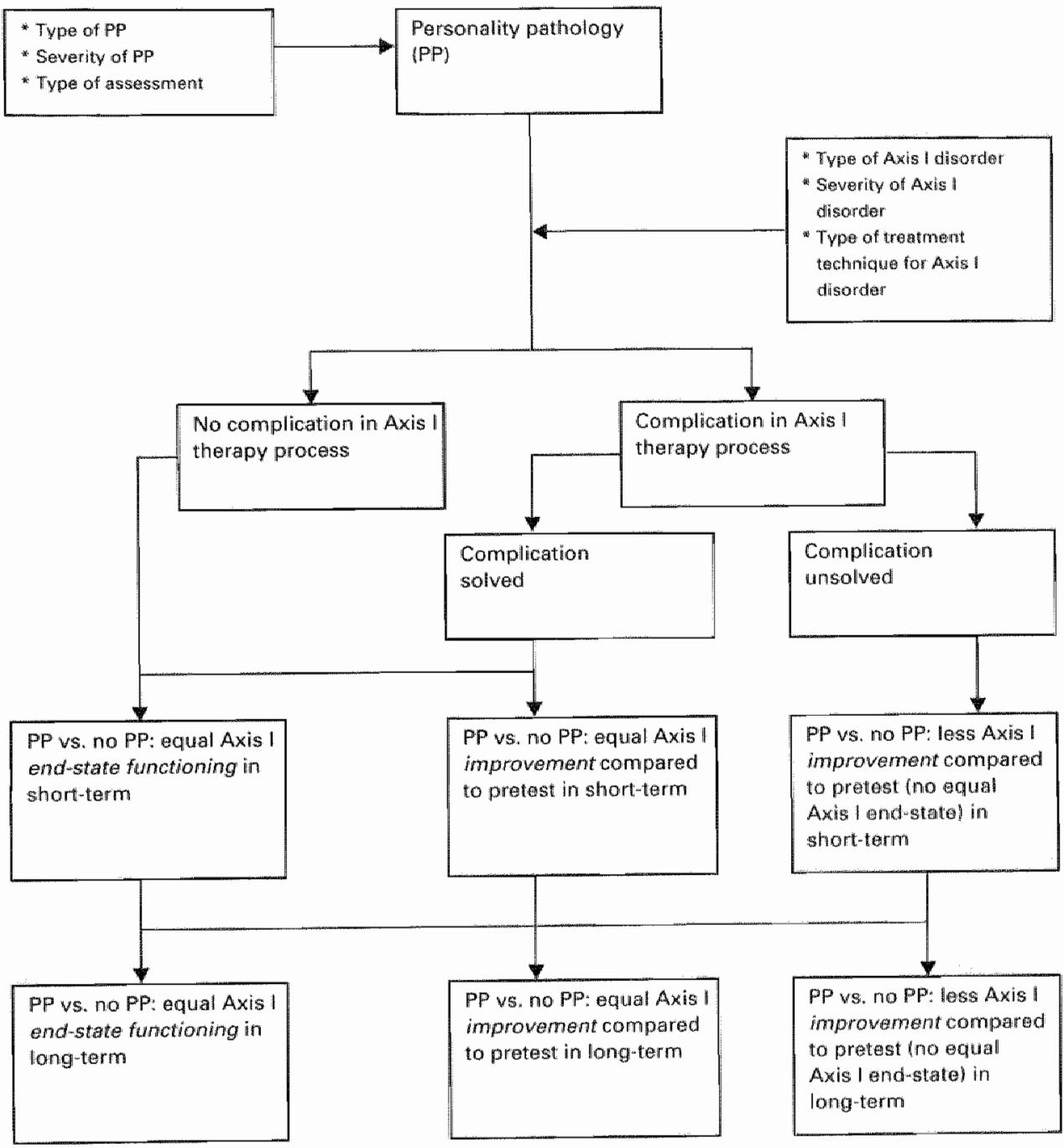

FIGURE 1

Pathways for personality pathology $(\mathrm{PP})$ to affect treatment outcome via treatment process 
Patients with Axis I panic disorder and obsessive compulsive disorder (section 3.1 and 3.2) were followed up until 6 months after the standardized therapy had finished, and the promising short-term results maintained. However, we do not know how the Axis i complaints evolve after those 6 months. Note that, for ethical reasons, patients received additional CBT after completion of the standardized treatment if clinically indicated. However, there were no indications for patients with concomitant PDs to receive more additional treatment, as an explanation for maintaining the results up until the 6 months follow-up.

Our three studies (section 3.1 and 3.2) showed no robust megative impact of $\mathrm{PP}$, assessed by a semistructured interview, on CBT outcome (i.e. amount of improvement) in anxiety disorders, panic disorder and obsessive compulsive disorder in particular. Similarly, two other Dutch studies found no negative impact of PP on change after CBT for social phobia (Mersch, Jansen, \& Arntz, 1995; Van Velzen, Emmelkamp, \& Scholing, 1997) and an American research group found no negative impact of PP on CBT for generalized anxiety disorder (Sanderson, Beck, \& McGinn, 1994). In other words, for all Axis I anxiety disorders, except for posttraumatic stress disorder and simple phobia, it has been demonstrated, at least in one study, that CBT was equally effective in patients with and without PDs. In all of these studies, PP was assessed by independent raters administering a semistructured interview before start of the Axis I treatment. Therapists and patients were kept blind to the PP diagnoses. This type of design has several strengths. First, semistructured PP interviews are reliable instruments with adequate face validity. Second, therapists (and the patients) should be kept blind for the PP diagnoses, because this information might facilitate negative beliefs about Axis I treatment outcome in advance. Third, and most importantly, in prospective designs, the patient's progress in Axis I complaints cannot influence the process of diagnosing PP (more PP might be diagnosed in therapy failures, because of the therapeutic failure). If we want to study the impact of PP on Axis I outcome, PP should be defined independent of Axis I outcome.

Including our own studies (section 3.1 and 3.2), 19 studies were traced that have focused on the impact of one or more PP variables on CBT outcome for anxiety disorders (Table 1). Note that differential effects (no effects, positive effects and/ or negatieve effects) can be reported within a study because the study included more than one PP variable and/or short- and long-term outcome and/or several outcome variables. A rough comparison of 8 'well-designed' (prospective design and [semi]structured interview for PP, see Chapter 4 for the arguments of these criteria) and 11 'less well-designed' studies, reveals the following. Of the 'welldesigned' studies, $100 \%$ reported no impact and/or a positive impact of one or more PP variables and only $50 \%$ of those studies reported also a negative impact of one or more PP variables. Of the 'less well-designed' studies, only $64 \%$ reported no impact and/or a positive impact of one or more PP variables, whereas $64 \%$ of those studies reported a negative impact of one or more PP variables (Table 1). Axis l end-state functioning, without correction for pretest scores, was left aside. In other words, 'well-designed' studies show less evidence for a negative impact of PP variables on CBT outcome for anxiety disorders than 'less well-designed' studies. ${ }^{3}$. These findings suggest that improvement in methodology fades away the negative association between $\mathrm{PP}$ and $\mathrm{CBT}$ treatment outcome in anxiety dis- 
orders. If there really is a negative association, one would expect this association to become more clearly by improving the methodology. Apart from methodology; differences in study results might also be caused by type and severity of the Axis $I$ anxiety disorder, type and quality of CBT, and type and severity of PP. Note that the "well-designed' studies are generally of a more recent date. New developments in CBT and knowledge about PP may have diminished the detrimental effects of PP on CBT for anxiety disorders. In short, more "well-designed' studies are needed to confirm the non-negative association between $P P$ and $C B T$ outcome in anxiety disorders, independent of type and severity of anxiety disorder, type of CBT, and type and severity of PP.

\section{TABLE 1}

Studies addressing the impact of personality pathology on outcome of cognitive and behavioral therapies for anxiety disorders

\section{Sterdy}

Dreessen et all. 1994 (Stuidy 1/

Chambless et al.. 1995

Dreessem et al., 1997

Mersch al., 1995

War Velzen et al, 1995

Feske et all., 1996

Sandersion et al., 1994

Dreessen et al., 1994 (Study 2)

Chambless et al, 1992

Clär et al., 1992

Marchand et al., 1993

Kejjers et al., 1994

Rathus et al., 1995

Rabavillias, 1979

Waughan \&eech, 1985

Mirichiello et al., 1987

Steketee. 1990

Fals-Stewart \& Lucente, 1993

Turner, 1987

Axis l anxiety
disorder
PAD
PAD
OCD
SPH
SPH
SPH
GAD
AnY AD
PAD
PAD
PAD
PAD
PAD
OCD
OCD
OCD
OCD
OCD
SPH
P

\section{mpact on Axis I treatrment outcomeb}

yes
yes $c$
yes c
yes c
yes $c$
yes d
yes d
yes $c$
no
no
no
no
no
no
no
no
no
no
no

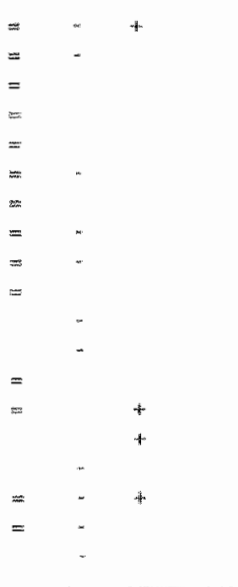

a Studies were considered well-designed if PP was assessed by a (semilstructured intorviow in a prospective design

b Axis I and state functioning, without correction for prettest scores was left aside

Axis therapists were kept blind for the outcome of the PP intervitew

d Not reported whether Axis I therapists were kept blind for the ouncome of the PP intarvilow

$P A D$, panic disordar

OCD, obsessive compulsive disorder

SPH, social phobia

GAD, gemeralized anxiety disorder

$A D$, anxiety disorder

- denotes no impact of PP variables on Axüs I improvement

- denotes negative impact of PP variables on Axis I improvement

* denotes posilive impact of PP variables on Axis 1 improwement

In Chapter 4 of the present thesis, all studies were reviewed that addressed the impact of PP on amy treatment for Axis I anxiety disorders. Two main selection criteria were used that studies had to meet to be included in the review. The study design had to be prospective and a (semi)structured interview had to be used for 
the assessment of PP. From the selected studies it was concluded that in general PP had no robust negative impact on results of therapies for anxiety disorders. Based on research so far, it can only be hypothesized that some specific PP variables, such as concomitant avoidant, schizotypal, and obsessive compulsive PP, affect treatment outcome of anxiety disorders negatively (see Chapter 4 for the evidence supporting this conclusion).

To our knowledge, no other study, except our own study described in section 3.3, has studied the impact of PP on the Axis I therapy process directly. PP, assessed by the SCID-II, was only weakly related to more complications in the Axis I therapy process and to more effort of the therapist, as reported by the therapist. Moreover, SCID-II derived PP Scores were unrelated to efficacy of therapist effort or the ability of the therapist to stop interference by the personality of the patient, as reported by the therapist. The data suggest that PP, as assessed by a semistructured interview by an independent rater, does not au tomatically result in a complicated Axis If therapy process in outpatients treated with CBT for their anxiety disorders. Furthermore, according to the therapists, complications arisen can be handled successfully, so Axis I therapy outcome will not necessarily be less in patients with compared to patients without PP because of complications in the Axis I therapy process.

When PP was estimated by the therapist in the course of treatment, instead of by the SCID-II by an independent rater before start of the therapy, a completely different picture arose: PP was strongly related to more complications in the Axis $I$ therapy process and more therapist effort, as reported by the therapist. Moreover, PP was related to less efficacy of therapist effort and less ability to stop interference by the personality of the patient, as reported by the therapist. It is tempting, but premature, to conclude from these data that therapists erroneously have attributed complications in the Axis I therapy process to concomitant PJ. But in line with this premature conclusion it can be argued that therapists who focus mainly on Axis I problems will have little detailed knowledge of the patient's personality functioning throughout life. Lack of information might lead to invalid PP evaluations. This may be true, but then it can be questioned whether study of long-term functioning is the only way to evaluate PP. Since the validity of the PP assessment instruments and most importantly the validity of the $\mathbb{P}$ ? construct is still a matter of research, we should not assume too easily that the SCID-II diagnoses are valid and the therapist evaluations of the personality pathology are invalid. It may turn out to be the other way around: SCID-II diagnoses being invalid and therapist evaluations being valid. Or, both the SCID-II and therapist evaluations may turn out to be invalid. Lastly, both SCID-II and therapist evaluations may turn out to be valid even though they are different, because they refer to different aspects of the same construct.

It is important to bear in mind that therapists were asked to judge on the personality of the patient, without administering a structured or unstructured clinical interview for $\mathbb{P P}$. So, the most important sources of information are the patient's behavior in therapy and whatever the patient reports spontaneously. Therefore, therapists may have included so-called temporary Pp diagnoses (i.e. features that look like PP, but that occur predominantly during episodes of Axis I mental disorders) next to real PP diagnoses that are typical for the person's long- 
term functioning, Axis I episodes included. If features occur exclusively during an episode of Axis I disorder, theoretically they could be considered as subclinical personality characteristics that flourish as a consequence of life stress. It may turn out that the PP construct is most valid when temporary PP features are included. In that case, therapists may turn out to be a good source of information in diagnosing PP. Whatever the case, therapists should also be imbued with other causes of complications in Axis I therapies besides PP, such as severity of the Axis I disorder, reinforcers of the Axis I problem behavior, and the impact of concomitant psychosocial and envirommental problems. Last but not least they should be critical towards their own therapeutic approach (e.g. did they offer a plain rationale to the patient? Is the exposure to the feared situation gradually enough? Are the instructions well understood by the patient?)

\section{Explanations for Discrepancy between Empirical Findings and Clinical Intuition}

Many studies have reported no negative impact of PP on treatment outcome of Axis I anxiety disorders (see Appendix 1, Chapter 4), which goes against the clinical intuition. Clinicians generally believe that PP affects treatment outcome negatively. How can this discrepancy be explained? Several factors may be responsible.

1. Clinicians in attendance are not blind to the Axis I therapy process and outcome. A complicated Axis I therapy process with disappointing results might easily incite them to diagnose false positive $\mathrm{PP}$ diagnoses during or at the end of therapy. Indeed, results of our study in section 3.3 seem to support this supposition: therapist derived PP scores correlated highly positively with complications in the Axis I therapy (as reported by the therapist), whereas SCID-II derived scores did not. As mentioned before, validity of the PP assessment instruments and the validity of the PP construct is still a matter of research, and we should not assume too easily that the SCID-II diagnoses are valid and the therapist PF evaluations are invalid (see allso point 11).

2. Clinicians who have negative assumptions about Axis I treatment outcome in advance, because the patient has been diagnosed with concomitant PP, might treat the patient with less effort and persuasiveness. The patients will respond with less improvement, which will confirm the clinician's negative assumptions. The negative assumptions might tally with the final outcome, but as a self-fulfilling prophecy. No study could be traced that has explicitly addressed this issue in patients with PDS, but there are many analogue studies showing that success in treatment varied with the attitude of the therapist toward the treatment and/or results (see review by Shapiro \& Morris, 1978).

3. Possibly, clinicians do not properly differentiate between PP and Axis 1 pathology (e.g. anxiety that goes together with Axis I disorders is different from anxiety that goes together with PP). In other words, they may see only little improvement in patients with severe $\mathrm{PP}$, because the PP does not improve spectacularly, and conclude that the Axis I therapy doesn't work.

4. Therapists may have difficulties to put aside the deep-rooted clinical notion that PP affects treatment outcome negatively (the 'exceptions prove the rule' principle). Such an idea may have hold for the past but not for the present anymore. Therapy for anxiety disorders and knowled ge about PP may have reached such a degree of development, that PP do not inevitably interfere with the treatment for anxiety disorders. 
5. New developments regarding treatment of anxiety disorders and knowledge about PP that may prevent PP from interfering with the treatment of Axis I anxiety disorder may be largely restricted to centers that are involved in empirical research. Consequently, clinicians in other centers may still experience more difficulties in the treatment of Axis I anxiety disorders in patients with concomitant PDS.

6. The clinical intuition may be true for Axis I disorders, other than anxiety disorders, such as a depressive disorders, eating disorders or substance-related disorders.

7. Clinicians may diagnose $\mathrm{PP}$ because they experience treatment interference of just one or two DSM Axis if features (e.g. 'being reluctant to engage in any new activities because they may prove embarrassing' or 'being preoccupied with doubts about the loyalty or trustworthiness of others, including the therapist'). Empirical studies usually evaluate less specific variables and in that way the negative effect of isolated features may be obscured. Similarly, potential. negative effects of subtypes within the PD categories may be obscured. Subtypes within $P D$ categories may for instance evolve from differences in childhood and adolescence experiences (e.g. borderline PD with/without sexual abuse) or follow from the polythetic format of the DSM classification system (e.g. dependent PD with/without difficulties in making everyday decisions; American Psychiatric Association, 1994). The importance of evaluating subtypes is demonstrated by a study in opiate addicts (Woody, McLellan, Luborsky, \& O'Brien, 1985). Overall, it was found that patients with concomitant antisocial PD were the least improved at the end of the study. However, patients with concomitant antisocial PD who were also depressed, improved as much as other non-antisocial subjects. Further examination suggested that the ability to form a therapeutic alliance characterized the patients with an antisociall PD who improved (Gerstley et al., 1989).

8. Some PDs are infrequently diagnosed in patients with anxiety disorders (e.g. antisocial and narcissistic PD) and therefore the optimistic results from the empirical studies may be restricted to the more frequently diagnosed PDS in this population (e.g. Cluster C PDs).

9. Severity of PP can be hypothesized to be an important factor in predicting Axis I treatment outcome. So tar $_{r}$ studies have only used the sum of PD diagnoses or the sum of traits as an indicator for severity. However, severity of single traits and severity of single PDs (and not just counting the number of traits within a specific category), has never been studied. Clinicians will correctly argue that this is an important issue for further investigation.

10. Most of the empirical studies focus on short-and middle term outcome only. Long-term studies may show that patients with concomitant PDs have difficullies to maintain the progress made in the Axis I therapy for the anxiety disorder.

11. Validity of the PP assessment instruments and the PP construct is still a matter of research. It may turn out that the instruments used in the empirical studies failed to assess validly the diagnostic criteria or that the diagnostic criteria themselves may be invalid. 
What can be concluded from the above? First, clinicians need to change their view: they cannot continue to assume that PP will affect treatment of Axis I anxiety disorders negatively. On the contrary, they should be open-minded about what to expect from Axis I therapy in patients with concomitant PP and they should be careful in attributing therapy failure to (assumed) concomitant PP. And, of course, they need to keep up with treatment-developments in the field of Axis I and Axis II disorders. Second, results of the studies are incomplete: further research is needed into more specific PP variables (e.g. subtypes within PD categories and specific traits). Severity of pP should be taken into account (and not only sumscores) and. impact of PP on longterm Axis I outcome needs to become a special focus of attention. Last but not least, efforts to improve on validity of PP assessment instruments and PP constructs should be continued.

\section{The role of cognitive theory in studying personality disorders}

Theoretical frameworks, such as the cognitive theory of PDs (e.g. Beck et al., 1990), provide guidelines for development of assessment instruments and, most importantly, they direct the processes for the validation of assessment instruments (e.g. theory predicts association with other variables) (Klein, 1993). Further, theory also provides guidelines for building hypotheses about etiological factors, comorbidity factors, effectiveness of treatment, treatment compliance, treatment outcome and future course (Klein, 1993).

As outlined in the general introduction, cognitive theory of PDs (e.g. Beck et al., 1990; Young, 1990) is one of the main theoretical orientations in the PD literature (Klein, 1993; Shea, 1993). The theory explains etiology (e.g. specific maladaptive parent-child interactions or intrusive events) and maintenance of PDS (e.g. schemaconfirming information processing biases) and offers a treatment model for PDS (e.g. schema-focused therapy). Moreover, the specific cognitive profiles of each PD indicate specific problems to be expected in the treatment of Axis I disorders (e.g. non-compliance to homework assignments because of the belief that it is better to do nothing than to make a mistake).

Research driven by the cognitive theory of pos has been scarce, and this theory is far from being properly validated. Some early steps have been taken in developing and evaluating PD belief questionnaires (e.g. section 5.1 of this thesis), in studying information processing biases as predicted from the theory (e.g. section 5.2 of this thesis), and in studying efficacy of cognitive therapy to change underlying maladaptive beliefs (e.g. case examples in Beck et al., 1990; Arntz \& Dreessen, 1993). To the best of the author's knowledge, no study has yet been carried out to study the impact of specific PD beliefs on treatment process or outcome of Axis I disorders. As stressed in chapter $4_{t}$ the impact of more specific $\mathbb{P P}$ variables on treatment outcome of Axis I anxiety disorders need to be studied. Cognitive theory can be very useful in determining such specific theory-based variables.

Our study on schema-congruent information processing biases in avoidant PD, presented in section 5.2 , must be seen as a venture in a relatively unexplored area. Our primary aim was to develop and to test the experimental task. This pilot study, using a non-clinical sample and a self-report screening questionnaire, was 
designed to prepare a controlled experiment with a clinical sample, properly screened for Axis I and II disorders by clinicians using well-known semistructured interviews.

Results of the pilot study were not completely in line with the predictions from the cognitive theory of the avoidant PD: DSM-III-R avoidant PP correllated with avoidant belief ratings, but was not associated with the predicted biases in the information processing (Note that DSM-III-R avoidant PP score correlated highly with most of the scores on other DSM-III-R PP scales, which suggest that the SCID-IIQ measured general PP without significantly discriminating between subscales). Avoidant belief ratings, on the other hand, were indeed associated with the predicted biases in the information processing. The pilot study does not allow to draw final conclusions on the specificity of this latter effect, but the finding clearly warrants further study.

In conclusion, the advantages of theory for the study of PDs are obvious. Cognitive theory lends itself readily to develop and evaluate assessment procedures and it can guide researchers to define a priori hypotheses about etiology. maintenance, treatment compliance and outcome in Axis I and Axis in therapies.

\section{Suggestions for further research}

Throughout the thesis and in this current chapter several suggestions for further research were given or implied. Below they will be summarized.

1. The short-interval test-retest approach should be used more frequently in studying interrater reliability of (semi)structured interviews for the assessment of PP. Following our interrater reliability study (section 2.1), more research is needed, first, to replicate our findings using the SCID-II for the DSM IV PDS, and second, to evaluate the PD variables, that could not be analyzed in our sample (schizoid, narcissistic, and antisocial dimensional PP variables and most of the categorical full-blown PDs).

2. Following our attempt to determine what source of information is more valid in the assessment of PP, informants or patients (section 2.2), more research is needed to clarify this issue of validity. A sensible approach would be to study validity of patient reports separately for every $\mathrm{PD}$. Validity of patient reports on PP may be restricted to a subset of PDs (e.g. narcissistic and antisocial PP may be difficult for the patient to acknowledge). It would also be interesting to study the validity of reports by patients who are just recovered from their Axis I disorders. In that way, the hypothesized distorting effect of state anxiety and depression is minimized.

Furthermore, treatment responsivity to Axis I therapies may be an interesting external criterion, against which to compare patient and informant derived diagnoses. If informant derived PP diagnoses are found to relate to Axis I treatment outcome, and patient derived Pp diagnoses are not, this would be an indication, but no conclusive indication, for the validity of informant derived pp diagnoses. More external criteria would be needed to confirm this indication, because informants may report difficult behavior which is not necessarily synonymous with PP.

From our studies (chapter 3) and from other studies (chapter 4) on the impact 
of PP on treatment of anxiety disorders it can be concluded that patient derived PP diagnoses were generally unrelated to the outcome of Axis I anxiety disorder therapies. Only one study could be traced that evaluated the relationship between informant derived PP diagnoses and treatment responsivity in Axis I anxiety disorders (Steketee, 1990). Similarly to patient derived Pp diagnoses, for the most part these informant derived PP diagnoses were unrelated or positively rellated to Axis I treatment outcome. Passive aggressive traits were negatively related to treatment outcome. More of such informant based studies are needed.

3. In studying the impact of PP on treatment outcome of Axis I anxiety disorders, many suggestions for further research can be made. First, some anxiety disorders are understudied, such as the generalized anxiety disorder and the posttraumatic stress disorder. But the other anxiety disorders also need further attention in order to clarify the divergence in findings across studies.

Second, future studies should focus on specific Pp variables, such as individual PDs or traits (e.g. schizotypal PD in patients with obsessive compulsive disorder, see section 2.2), or specific maladaptive beliefs (see General Introduction of this thesis: Cognitive Theory of PDs). Since there are a lot of specific PP variables, clear hypotheses should guide the selection.

Third, studies need to focus more on dimensional. PP variables, without excluding the categorical PP variables.

Fourth, severity of PP should be taken into account more properly. Hitherto, severity is operationalized as the number of PD traits or the number of $\mathrm{PD}$ diagnoses. But, that leaves aside the severity of single traits and in that manner the qualitative severity of individual PDs. Future studies should include qualitative indices of severity of PP, for instance by rating the severity of single traits on a three point Lickert scale.

Fifth, data of the drop-outs need to be included in the analyses of Axis I treatment outcome.

Sixth, long-term follow-up studies are needed to find out whether patients with PP maintain the parallel improvement and whether they ever reach the end-state functioning of patients without PP (see final pathway presented in Figure 1).

Seventh, the possibility that the effect of pp interacts with the type of intervention used for the treatment of anxiety disorders (e.g. pharmacotherapy vs. cognitive behavior therapy) deserves further study in carefully controlled designs.

Finally, it would be very interesting to study the impact of therapist's beliefs about Axis I treatment responsivity in patients with concomitant PP.

4. Following our study on the impact of PP on the Axis I process of cognitive behavior therapy of anxiety disorders, much more research is needed. First, future studies need to focus more on the impact of specific PP variables (taking into account the severity), and less on general pep variables.

Second, studies are needed to better understand how PP can complicate the therapy process, and how therapists can handle this complication successfully. Third, it would be interesting to use independent therapy process measures, next to process evaluation made by the therapist, and to evaluate the therapy 
process a second time later on in the therapy (we assessed the process between session 6 and 7: complications may arise later on in the therapy process or on second thoughts the therapist may conclude that complications cannot be handled successfully).

Finally, we focused on cognitive behavior therapy for anxiety disorders. Of course, research should also focus on other Axis I disorders and other therapies.

5. Research on the validity of the cognitive model for pos has just been started. The content of the cognitive profiles and the presumed cognitive distortions as well as their relation to dysfunctional behavior and distress need to be verified for all individual PDs. Three new research areas in the field of PDs are: 1. research on cognitive content measures for PDs (e.g. PDBQ; Dreessen, Arntz, \& Weertman, 1996),

2. research on schema-congruent information processing biases in PDs, and

3. research on cognitive therapy for PDs.

\section{Recommendations for clinical practice}

The present thesis results in a number of clinical recommendations. Below they will be summarized point by point:

1. The SCID-II is a fairly reliable instrument for the assessment of $\mathrm{PP}$ in clinical practice and is a good supplement to the common practice of clinical personality disorder diagnoses.

2. No definite answer can be given yet on the validity of the SCID-II, or any other standardized or unstandardized measure of PP. Further research has to prove the validity of patient reports on PP.

3. Patients with PP should not be excluded from cognitive or behavior therapy for their Axis I anxiety disorder. PP, as measured by the SCID-II, has no excessive negative impact on therapy process and final outcome in the treatment of Axis I anxiety disorders. Future research may show that there are some specific PP variables that predict less well treatment outcome.

4. Attributing therapy failure to (assumed) concomitant PP should be approached with caution. Other causes of failures need to be considered profoundly and therapists should be able to define the PP by criteria other than the therapeutic failure alone.

5. Therapist should be open-minded about what outcome to expect from cognitive behavior therapy for Axis I anxiety disorders in patients with concomitant PP. Meanwhile, they can anticipate problems to be expected in therapy and try to deal with them successfully.

6. Knowledge about $P$, such as for instance the cognitive profile of individual PDS, may help the therapist to anticipate, to understand, and to cope with difficulties that tend to limit therapy outcome. 


\section{notes}

1 The polythetic format of the DSM-III-R/DSM-IV PDs is less of a problem when the internal consistency of the PD category is high. The internal consistency of the DSM-II-R PD categories has been found to range from fair to good, using the SCID-II to assess the individual traits Uansen, Arntz, Merckelbach, \& Mersch, 1994).

2 In order to distinguish between full-blown DSM PDs and other PD variables (e.g. dimensional traitscore), the term personality pathology is introduced (PP), indluding both categorical and dimensional PD variables.

3. A more detailed comparison between the "well-designed" and the "less well-designed" studies would support this conclusion even more, because most of the studies that evaluate more than one PP variable mainly report positive or no elfects. 


\section{References}

American Psychiatric Association (1968). Diagrostic and Statistical Mantal of Mental Disorders (2nd ed.). Washington, DC: Author.

American Psychiatric Association (1980). Dingrnostic and Statistical Mamual of Mental Disorders (3rd ed.). Washington, DC: Author.

American Psychiatric Association (1987). Diagnostic and Statistical Manual of Mental Disorders (3rd. ed. rev.) Washington, DC: Author:

American Psychiatric Association (1994). Diagnostic and Statistical Manual of Mental Disorders (4thed.). Washington, DC: Author.

Arntz, A. \& Dreessen, L., (1993). Cognitieve gedragstherapie bij persoonlijkheidsstoornissen. Directieve Therapie, 1 , jaargang 13, 5-26.

Arntz, A. (1987). Adapted Dutch version of the SCFD Persondity Qhestionarire. Maastricht University: Author.

Amtz, A. (1993). Theatment of borderline personality disorder: a challenge tor cognitive behaviour therapy. Behaviour Research and Therapy, 32, 419-430.

Arntz. A., Bögels, S. \& Hoekstra, R. (1992-all. Revisie van de Nederlondae vertaling wan het Gestructureerd Klinisch Interview voor DSM-IM-R (SCID) [Revision of the Dutch translation of the Structured Clinical Interview for DSM-III-R (SCID)]. Maastricht Liniversity: Author.

Amtz, A., Dietzel, R., Dreessen, \& L. (1997). Specificity and stability of borderline assumptions and their relationship with etiological factors. Manuscript submitted for publication.

Arntz, A., van Beijsterveldt, B., Hoekstra, R., Hofman, A., Eussen, M., \& Sallaerts, S. (1992-b). The interater reliability of a Dutch version of the Structured Clinical Interview for DSM-III-R Personality Disorders. Acta Psychiatrica Sandinarica, 85, 394400.

Arrindell, W. A. \& Ettema, H. (1981). Dimensionele structuur, betrouwbaarheid en validiteit van de Nederlandse bewerking van de Symptom Checklist (SCL-90). [Dimensional structure, reliability and validity of the Dutch version of the Symptom Checklist (SCL-90)]. Nederlands Tijdschrift woor de Psycliologie, 36, 77-108.

Baer, Lo, Jenike, M. A., Black, D. W. Treece, C, Rosenfeld, R., \& Greist, J. (1992). Effect of Axis II diatgnoses on treatment outcome with clomipramine in 55 patients with obsessive-compulsive disorder. Archives of General Psychatry, 49, 862-866.

Baer, L., Jenike, M. A., Ricciardi, J. N., Holland, A. D., Seymour, R. J., Minichiello, W. E, \& Buttolph, M. L. (1990). Standardized assessment of personality disorders in obsessive-compulsive disonder. Archizes of General Psychiony, 47, 826-830.

Beck, A. T. (1976). Cognitize Therapy and the Emotional Disorders. New York: International Universities Press.

Beck, A. T. (1990). Belief Questionnaire. Unpublished manuscript.

Beck, A. T. Freeman, A., \& Associates (1990). Cognilive Therapy of Personality Disorders. New York: Guilford.

Beck, A. T., Rush, A. J Shaw, B. F. E Emery, G. (1979), Cognitive Therapy of Depression. New York: Wiley.

Benjamin, L. S. (1993). Mnterpersanal Dingnosis and Treatment of Persandaly Disorders. New York: Guilford.

Bernstein, D. P., Kasapis, C., Bergman, A., Weld, E., Mitropoulou, V., Horvath, "T., Klatr, H. M., Silverman, J., Siever, L. J. (1997). Assessing Axis II disorders by informant interviews. Joumal of Persomality Disonders, 11, 158-167. 
Black, D. W., Wesner, R. B., Gabel, J., Bowers, W., Monahan, P. (1994). Predictors of short-ferm treatment response in 66 patents with panic disorder fowmal of Afectroe Disorders, 30, 233-24].

Black, D. W. Yates, W. R., Noyes, R., Pfoh, B., Kelley, M. (1989). DSM-II personality disorder in obsessive compulsive study volunteers: As controlled study. Journal of Personality Disorders, 3,58 62.

Bouman, T. K. (1987). The Menswement of Depression with Qmestiomaines. Unpublished doctoral dissertation, Groningen Uniwersity, The Netherlands.

Brooks, R. B., Ballazar, P. L., McDowell, D. E. Murjack, D. I. Bruns, J. R. (1991). Personality dlisorders co-occurring with panic disorder with agoraphobia. Jownal of Persondity Disoriters, 5,328 336.

Bruch, M. A., Kanowit, N. G., \& Berger, P. (1988). Self-schema for assentiveness extending the validity of the self-schema construct. Joumal of Research in Personality, 22, 424-44.

Chambless, D. L, Renneberg, B., Fydrich, T., Goldstein, A. J., G Gracely, E. J. (1995). Axis I and Axis II comorbidity in agoraphobia: prediction of treatment outcome. Unpublished ntanuscript.

Chambless, D. L., Renneberg, B., Goldstein, A., R Gracely, E. J. (1.992). MCML-diagnosed personality disorders among agomphobic outpatients: prevalence and relationship to severity and treatment outcome. Journat of Anxiety Disorders, 6, $193-211$.

Cllair, A. L., Oei, T. P. S. \& Evans, L. (1992). Personality and treatment nesponse in agoraphobia with panic athacks. Comprehensioe Psychatry, 33, 310-318.

Clark, D. M. \& Salkovskis, P. M. (1991). Cognatwe Therapy for Panic and Hypochowdrinsis. New York: Pergamon.

Cohen, ). (1960). A coefficient of agreement for nominal scales. Ed wational and Psychological Measurement, $20,37-46$

Cooper, ]. (1970). The Leyton Obsessional Inventory. Psychological Medicine, 1, 48-64.

Cronbach, L. \& Meeh., P. (1955). Construct validity in psychological tests. Psychological Bulletin, 52, $281-302$.

Davidson, I. R. T., Kudler, H. S., Saunders, W. B., Erickson, L., Smith, R. D., Stein, R. M., Lipper, S., Hammett, E. B., Mahomey, S. L., \& Cavenar, I. O. (1993). Predicting response to amitriptyline in postlatamatic stress disorder. Anerican Jownal of Psychintry, 150, 1024-1029.

Dowson, J. H. (1992). Assessment of DSM-III-R personality disonders by self-report questionnaine: the role of informants and a screening test for commorbid personality disorders (STCPD). British Jowm of Psychiatry, $161,344-352$.

Dreessen L. \& Arntz, A. (1998 a The impact of personaly disorders on meatment outcome of andety disonders: Bestevidence synthesis. Behandw Reseanch and Theray, 36, 483-504.

Dreasen, L, \& Arntz, A. (1998-b). Short-interwal test-retest intervater reliabilty of the Structured Clinical Interview for DSM-II-R Personality Disorders in outpatients. fownal of Personality Disorders, 12 , $138-148$.

Dreessen, L. E Arnlz, A. (1998-c). Personality disorders have no excessively negative impact on the therapy process in the cognitive behavioral treatment of Axis I anxiety disorders. Clonical Pychology

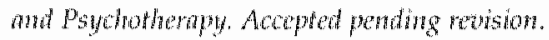

Dreessen, L. Amtz, A. \& Weertman, A. (1996). Personality Disorier Belief Questionmaire (version $1.0 \%$. Mastrich University: Author.

Dreescen, L., Arntz, A., Hendriks, T., Keume, N. \& Van den Hout, M. A. (1998-b), Atoidant personality

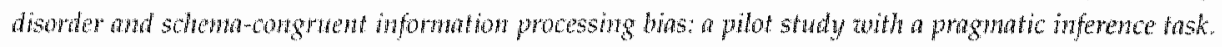
Manuscript submitted for publication.

Dreessen, L., Amtz, A., Luttels, C., \& Sallaerts, S. (1994). Personality disorders dio mot infuence the

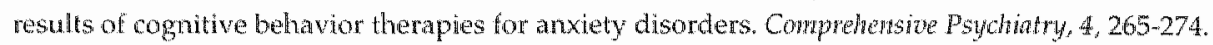


Dreessen, L., Hildebrand, M. \& Amtz, A. (1998-a). Patient-intormant concordance on the Struchued Clinical Interview for DSM-III-R Personality Disorders (SCID-II) Jourmal of Peromality Disorders, 12, 149-161.

Dreessen, L., Hoekstra, R., \& Arntz, A. (1997). Personality disorders do not influence the results of cognitive and behavior therapy for obsessive compulsive disorder. Jotumal of Anxiety Disorders, 11 . 503-521.

Edell, W. (1981). The borderline syndrome index. Clinical validity and utility lowrul of Nerwous and Mental Disense, 172, 254-263.

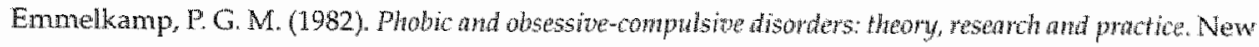
York: Plenum.

Emmelkamp, P. G. M., Visser, S., \&. Hoekstra, R. J. (1988). Cognitive therapy es. exposure in vivo in the treatment of obsessive-compulsives. Cognitive Therapy and Resench, 12, 103-114.

Eussen, M., Arntz, A. \& Hoekstra, R. (1991). The tactorial validity of the SCDD II]. Unpublished raw data.

Fals-Stewart, W. Lucente, S. (1993). An MCMI cluster typology of obsessive-compuisives: n measure of personality characteristics and its relationship to treatment participation, compliance and outcome in behavior therapy. Joumal of Psychiatric Research, $27,139-154$.

Faravelli, C. Albanesi, G. (1987). Agoraphobia with panic attacks: 1-year prospective follow-tup. Comprehensioe Psychiatry. 28, 481-487.

Fennell, M. (1989). Depression. In: K. Hawton, P. M. Salkowskis, J. Kirk \& D. M. Clark (Eds. y, Cogntitue behaviour therapy for psychiatric problens: A practical guide (pp. 169-234). Oxford: Oxford University Press.

Feske, U., Perry, K. J, Chambless, D. L., Renneberg, B., \& Goldstein, A. I. (1996). Avoidant personality disorder as a predictor for treatment outcome among generalized social phobics. Journal of Persontily Disorders, 10, 174-184.

First, M. B., Spitzer, R. L., Gibbon. M, \& Williams, J. B. W. (1995-a). The Structured Clinical Interview for DSM-III-R personality disorders (SCID-ID). Part I. Description. Jourmal of Personality Disonders. 9. 83-91.

First, M. B., Spitzer, R. L., Gibbon, M. Willams, J. B. W. Benjamin, L. (1994). Structwad Clinical Interview for DSM-IV Axis II personality disonders (SCDD-D)(Version 2.01. New York: Biometrics Research Department, New York State Psychiatric Institute,

First, M. B., Spitzer, R. L., Gibbon, M. Williams, J. B. W., Davies, M., Borus, J., Howes, M. J., Kane J., Pope,fr., H. G. \& Rounsaville, B. (1995-b). The structured clinical interview for DSM-III-Re Personality Disorders (SCID-11), Part 11: multi-site test-retest reliability study, foumal of personnity Disorders, 9 , 92-104.

Fleiss, I. L. (1981). Statistical Methods for Rates and Proportions. New York: Wiley.

Fleiss, ]. L. (1986). The Design and Analysis of Clinical Experintents. New York: Wiley.

Foley, S.H., O'Malley, S, Rounsaville, B. Prusoff, B. A., \& Weissman. M. M. (1987). The relationship of patient difficulty to therapist performance in interpersonal psychotherapy of depression. joumal of Affective Disorders, 12, 207-217.

Fydrich, T., Schmitz, B., Hennch, Ch, \&E Bodem, M. (1996). Zuverlässigkeit und Gültigkeit diagnostischer Verfahren zur Erfassung von Persönlichkeitsstörungen [Reliability and validity in diagnosing

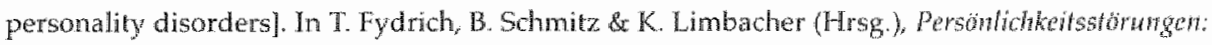
Dingrostitid und Psychotherapie (pp. 91-116). Weinheirn: Beltz.

Garfield, S. L (1994). Research on client wariables in psychotherapy. In A.E. Bergin S. S. Garfield, (Eds.), Handbook of Psychotherapy amd Behavior Change (4th ed.). New York: Wiley. 
Gerstley, L., McLellan, T., Alterman, A. I., Woody, G. E., Luborsky, L. \& Prout, M. (1989). Ability to form an alliance with the therapist: A posstble marker of prognosis for patients with antisocial personality disonder. Axterican fournat of Psychiatry, 146, 508-512.

Gorman, I. M., Liebowitz, M. R. Fyer, A. J. \& Stein, J. (1989). A neuroanatomical hypothesis for panic disorder. Americas Joumal of Psychatry, 146, 148-161.

Green, M. A. \& Curtis, G.C. (1988). Personality disorders in panic patients: Response to termination of antipanic medication. Journal of Personality Disorders, 2, 303-314.

Harris, R. J. \& Monaco, G. E. (1978). Psychology of pragmatic inference: information processing between the lines. Joumal of Experivnewtal Psychology, 207, 1-22.

Hennch, $\mathrm{C}$. Lauter, $\mathrm{G}$, \& Fydrich, T. (1994). Interrater and Test-vetest Reliability of the Germant Version of the Structured Clinical Interiziew for DSM-II-R Personality Disorders (SCID-II). Poster presented at the First European Congress on Disorders of Personality, Nijmegen, The Netherlands.

Hermesh, $H_{\text {. }}$ Shahar, A, \& Munitz, H. (1987). Obsessive-compulsive disorder and borderline personality disorder. American foumal of Psychiatry, 144, 120-121.

Hirschfield, R. M. A., Klerman, G. L., Clayton, P. J., Keller, M. B., MCDonald-Scott, P. \& Larkin, B. H. (1983). Assessing personality: effects of the depressive state on trait measurement. American yournal of Psychiating. 140, 695-699.

Hodgsen, R. I, \& Rachman, S. (1977). Obsessional-compulsive complaints. Behowiour Research and Therapy, 15, 389-395.

Hoekstra, R. J., Arntz, A., \& Hout, van clen, M. A. (1998). Exposure and/or cognitize therapy: differential effectiveness in obsessize compulswe disorder. Manuscript in preparation.

Hoffart, A. \& Martinsen, E. W. (1993). The effect of personality disorders and anxuous depressive comorbidity on outcome in patients with unipolar depression and with panic disorder and agoraphobia. Journal of Personality Disorders, 7, 304-311.

Holt, C. S., Heinberg, R. G. \& Hope, D. A. (1992). Avoidant personality disorder and the generalized subtype in social phobia. loumal of Abnormal Psychology 101, 318-325.

Horowitz, L. M., Rosenberg, S. E., \& Bartholemew, K. (1993). interpersonal problems, attachments styles, and outcome in brief dynamic psychotherapy. Joumal of Consulting and Clinical Psychology, $61,549-560$.

Hunt, C. \& Andrews, G. (1992). Measuring Personality Disorders: the use of self-report questionnaires. Journal of Personality Disorders, 6, 125-133.

Hurt, S., Hylet, S. Frances, A., Clarkin, J., \& Brent, R, (1984). Assessing borderline personality disorder with self-report, clinical interview, or semistructured interview. American fourmal of Psychiatry, 141. 1228-1231.

Hyler, S. E., Rieder, R. O., Williams, J. B. W, Spitzer, R. L., Lyons, M. \& Hendler, J. (1987), A comparison of clinical and self-report diagnoses of DSM-III personality disorders in 552 patients. Comprehensize Psychiarry, 30, 170-178.

Hyler, S. E., Skodol, A. E. Kellman, H. D., Oldham, J. M., kesnick, L. (1990). Validity of the Personality Diagnostic Questionnaine-Revised: comparison with two structured interviews. American Jouthal of Psychiatry, 147, 1043-1048.

Hylex, S. E, Skodol, A. E., Oldham, J. M., Kellman, H. D., \& Doidge, N. (1992). Validity of the Personality Diagnostic Questionnaire-Revised a replication in an outpatient sample. Comprehensize Psychiatry. $33,73-77$.

Jacobson, N.S., \& Truax, P. (1991). Clinical significance: a statistical approach to defining meaningful change in psychotherapy research. Journal of Consulling and Clinical Psychology, 59, 12-19.

Jansen, M. A., Amtz, A.w Merckelbach, H., \& Mersch, P-P. (1994). Personality disorders and features in social phobia and panic disonder Journat of Abnomal Psychology, 103, 391-395. 
Joffe, R. T. \& Regan, I. J. (1988). Personality and depression, Joumal of Psychatric Research, 22, 279.286. Johnson, M. K., Bransford, J. D. \& Solomon, S. (1973). Memory for tacit implications of sentences. Jourral of Experimental Psychology, $98,203-205$.

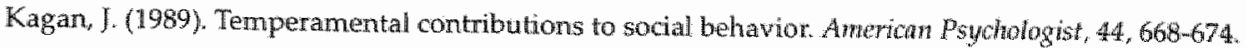

Keijsers, G. P. J. Hoogduin, C. A. L., \& Schaap, C. P. D. R. (1994). Prognostic factors in the behavioral treatment of panic disorder with and without agoraphobia. Behavior Therapy, 25, 689-708.

Klein, M. H. (1993). Issues in the assessment of personalify disorders. Journal of Personality Disorders, Supplement, Spring, 18-33.

Koster van Groos, G. A. S. (1985). Nederlandse vertaling pan het Gestruchureerd Klinsch Interoiew voor DSM-III-R (SCID) [Dutch transiation of the Structured Clinical Interview for DSM-III-R (SCID)]. Unpublished mantuscript.

Kranzler, H. R., Kadden, R. M., Babor, T. F, \& IRounsaville, B. J. (1994). Longitudinal, Expert, All Data procedure for psychiatric diagnosis in patients with psychoactive substance use disorders. The foumal of Nerwous and Mental Disense, 182, 277-283.

Kringlen, E. (1965). Obsessional neurotics. British Joumal of Psychiatry, $111,709-722$.

Landis, J. R. \& Koch, G. G. (1977). The measurement of observer agreement for categorical data. Biometrics, 33, 159-174.

Lo, W. H. (1967). A follow-up study of obsessional neurotics in Hong Kong Chinese. British Journal of Psychiatry, 113,823-832.

Loranger, A. W. (1992). A.re current self-report and interview measures adequate for epidemiological studies of personality disorders? Jourwal of Personality Disorders, 6, 313-325.

Loranger ${ }$ A. W., Sartorius, N., Andreoli, A., Berger, P, Buchlheim, P., Channabasavama, S. M., Coid, B., Dahl, A., Diekstra, R. F. W., Ferguson, B., Jacobsberg, L. B., Mombour, W., Pull, C., Ono, Y., \& Regier D. A. (1994). The International Personality Disorder Examination. Archives of General Psychiatry, 51, 215-224.

Loranger, A. W., Susman, V. L., Oldham, I. M., \& Russakoff, M. (1987). The Personality Disorder Examination: a preliminary report. Joumal of Personality Disorders, 1, 1-13.

Maier, W., Lichtermann, D., Klinger, T., Heun, R., Hallnayer, J. (1992). Prevalences of personality disorders (DSM-III-R) in the community. Jourtal of Personality Disorders, 6, 187-196.

Malow, R. M., West, J. A., Williams, J. L., \& Sutker, P. B. (1989). Personality disorders classification and symptoms in cocaine and opioid addicts. Journal of Consulting and Clinical Psychology, 57, 765-767.

Mancusa, D. M., Townsend, M. H., \& Mercante, D. E. (1993). Long-term follow-up of genertalized andiety disorder. Comprehensive Psychialty, 34, 441-446.

Marchand, A., Lebeau, I. \& Trudel, G. (1993). The wapat of personality disonders on the outcotre of a behavioural-cognitive treatwent for panic disorder with agoraphobia: A preliminary study. Paper presented at the 23rd European Congress of Behaviour and Cognitive Therapy, London, 1993.

Marks, I. M. \& Matthews, A. M. (1979). Brief standard self-rating scale for phobic patients. Behwour Research and Therapy, 17, 263-267.

Markus, H. \& Zajonc, R. B. (1985). The cognitive perspective in social psychology. In G. Lindzey and E. Aronson (Eds.), The Handbook of Social Pyychology, 3rd ed, Vol. 1 (pp. 137-230). New York: Random House.

Markus, H. (1977). Self-schemata and processing of information about the self. fowmtot of Personality and Sacial Psychology, $35,63-78$.

Martin, M., Ward, J. C. \& Clark, D. M. (1983). Neuroticism and the recall of positive and negative personality information. Beharvior Research and Therapy, 21, 495-503.

Mavissakalian, M. \&. Hamam, M. S. (1987). DSM-II personality disorders in agoraphobia. Il. Changes with treatment. Compreplensive Psychiatry, 28, 356-361. 
Mavissakaliar, M. Hamann, M. S. \& Jones, B. (1990). DSM-III personality disorders in obsessivecompulsive disorder: changes with treatrinent. Comptehensive Psychiaty. 31, $432-437$.

McNemar, Q. (1969). Psichological Sfatistics. New York: Wiley.

Mellman, T. A. Leverich, G. S, Hauser, P., Kramlinger, K., Post, R. M. \& Uhde, T. W. (1992). Axis II pathology in panic and affective disorders: relationship to diagnosis, course of illness, and treatment response Jourtal of Personality Disorders, 6, 53-63.

Mellsop, G. Varghese, F, Joshua, S. \& Hicks, A. (1982). The reliability of Axis Il of DSM-III. Anterican fow mal of Psychiatry, 139, 1360-1361.

Mersch, P.P. A., Jansen, M. A., \& Amtz, A. (1995). Social phobia and personality disorder: severity of complaint and treatment effectiveness. Jotmal of Personality Disorders, 9, 143-159.

Millon, T. (1987). Manual for the Millon Clinical Multaxial Intevtory-L. Minneapolis: National Compu* ter Systems.

Minichiello, W. E., Baer, L., \& Jenike, M. A. (1987). Schizotypal personality disorder: a poor prognostic indicator for behavior therapy in the treatment of obsessive-compulsive disonder. Journd of Anxiety Disorders, 1. 273-276.

Noyes, R, Reich, J. H., Suelzer, M., \& Christiansen, I. (1991). Personality traits associated with panic disorder: change associated with treatment. Comprehensive Psychiatry, 32, 283-294.

Noyes, R., Reich, J., Christiansen, I., Suelzer, M., Pfohl, B., \& Coryell, W. A. (1990), Outcome of panic disorder Relationship to diagrostic subtypes and comorbidity. Archires of Gereral Psychiatry. 47 . $809-818$

Numberg, H. G., Raskin, M., Levine, Ph. E., Pollack, S., Prince, R. \& Siegel, O. (1989). Borderline personality disorder as a negative prognostic factor in anxiety disorders. Journal of Personality Disorders, 3, 205-216.

O'Boyle, M. \& Self, D. (1990). A comparison of two interviews for DSM-III-R personality disorders. Psychinatry Research, 32, 85-92.

Oldham, I. M. Skodol, A. E., Kellman, D. Hyler S. E, Rosnick, L. \& Davies, M. (1992). Diagnosis of DSM-III-R personality disorders by two structured interviews: patterns of comorbidity. American fowrmal of Psychialry, $249,213-220$.

Ost, L. G. (1987). Applied relaxation: Description of a coping technique and review of controlled studies. Behaviour Research and Therapy. 25, 13-22.

Ouimette, P. C. \& Klein, D. N. (1995). Test-retest stability, mood-state dependence, and informantsubject concordance of the SCID-Axis II Questionnaire in a nondinical sample. Joumal of Personality Disorders, $9,105-111$.

Padesky, C. A. (1988). Schema-focused cognitive therapy: comments and questions. International Cognition Therapy Newsletter, 4, 5-7.

Padesky, C. A. (1994). Schema change processes in cognitive therapy. Clinical Psychology and Psychotherapy, $1,267-278$.

IPfohl, B., Blum, N. \& Zimmerman, M. (1995). Struchured Interoteto for DSM-IV Personality, SIDP-IV Department of Psychiatry, University of Lowa: Author.

Pilkonis, [P. A., Heape, C. L., Ruddy, I. \& Serrao, P. (1991), Validity in the diagnosis of personality disorder: the use of the LEAD standard. Psychological Assessment. Jownt of Consulting and Clinical Psychology, 3, 46-54.

『retzer, J. \&. Fleming, B. (1989). Cognitive behavional treatment of personality disorders. The Behnzior Therapist, 12, 105-109. 


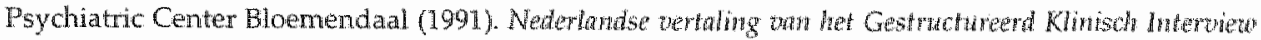

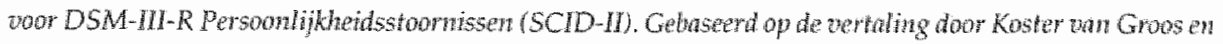
PC Blocmenthal nit 1987. Den Hang, PC Bloemendaal. [Dutch translation of the Structured Clinical Interview for DSM-III-R personality disorders [SCID-II]. Based on the translation by Koster van Groos and PC Bloemendaal from 19877. Den Haag, The Netherlands: Author.

Rabawilas, A. D., Boulougounis, J. C., Perissaki C., \& Stefanis, C. (1979). Pre-morbid personality traits and responsiveness to flooding in obsessive-compulsiwe patients. Behoviour Research and Therany, 17, 575-580.

Rathus, I. Sanderson, W. C. Miller, A. L. \& Wetzler, S. (1995). Impact of personality functioning on cognitive behavioral treatment of panic disorder a preliminary report. Jourtal of Personthity Disorders, 9,160 -168.

Rau, P. J. \& Goldfried, M. R. (1994). The therapeutic alliance in cognitive-behavior therapy. In A. D. Horwath \& L. S. Greenberg (Eds.), The Working Alinnce: Theory, Research and Practice. New York: Wiley:

Reich, I, (1990). The effect of personality on placebo response in panic patients. fownal of Nerows mat Merital Disease, 187, 699-702.

Reich, J. H. \& Green, A. I. (1991). Effect of personality disonders on outcome of treatment. The foum of Nerrous and Mental Disease, 179, 74-82.

Reich, J. H. \& Noyes, R. (1987). A comparison of DSM-III personality disorders in acutely ill panic and depressed patients. Joumal of Anxiety Disorders, I, 123-131.

Reid, I. H. \& Vasile, R. G. (1993). Effect of personality disorders on the treatment outcome of Axis I conditions: An update. The fowmal of Nerwons and Mental Disenst, 181, 475-484.

Reich, I. H. (1988). DSM-III personality disorders and the outcome of treated panic disorder. Americin Joumal of Psychiatry, $145,1149-1152$.

Reich, J. H., Noyes, R., Coryell, W. \& Hirschfield, R. R. (1986). The effect of state anxiety on personality measurement. Anterican Joumal of Psychiary, 143,760-763.

Reich, I., Noyes, R., \& Troughton, E. (1987). Dependent personality disorder associated with phobic avoidance in patients with panic disorder. Americon Joumal of Psychantm, 144, 323-326.

Renneberg, B., Chambless, D. L., Dowdan, D. J., Fauerbach, J. A. \& Gracely, E. J. (1992). The Structured Clinical Interview for DSM-III-R Axis II and the Millon Clinical Multiaxial Inventory: a concurrent validity study of personality disorders among anxious outpatients. Jownal of Personelity Disonders. $6,117-124$.

Repko, G. R. \& Cooper R. (1985). The diagnosis af personality disonder A companison of MMPl profils, Millon linventory, and clinical judgement in a workers compensation population. Journat of Clinical Psychology, 41,867-881.

Riso, L. P. Klein, D. N. Anderson, R. L., Oumette, P. C. \& Lizard, H. (1994). Concordance between patients and informants on the Personality Disorder Examination. American Joumal of Psychiatry. $151.568-573$.

Rogers, T. B., Kuiper, N. A., \& Kirker W. S. (1977). Self-reference and the encoding of personal information. poumal of Personality and Social Psychology. 35, 677-688.

IRojahn, K. \& Pettigrew, T. F. (1992). Memory for schema-relevant infomation a meta-analytic resolution. British Journal of Social Psychology, 31, 81-109.

Rosenberg, M. (1965). Saciety and the Adolescent Self-image. Princeton: Princeton University Press.

Safran, J. D., Segal. Z. V., Hill, C., \& Whiffen, V. (1990). Refining strategies for research on selfrepresentations in emotional disonders. Cognitize Therapy and" Resenrch, 14, 143-160. 
Sanderman, R, Eysenck, S. B. G. \& Arrindell, W. A. (1993). A cross-cultural comparison of personality traits: Astudy with the Eysenck Personality Questionnaire (EPQ) in Great Britain and the Netherlands. Unvphlished mamiscript.

Sanderson, W. C., Beck. A. T. \& McGinn, L. K. (1994). Cognitive therapy for generalized anxiety dism order: significance of comorbid personality disorders. Journal of Cogritive Psychotherapy: An International Quarterly, 8, 13-18.

Schmidt, N. B., Joiner, Th. E., Young J. E., \& Telch, M. J. (1995). The Schema Questionnaire: Investigation of psychometric properties and the hierarchical structure of a measure of maladaptive schemas. Cognitive Therapy and Research, 19, 295-321.

Schneier, F. R., Spitzer, R. L., Gibbon, M., Fyer, A. J. \& Liebowitz, M. R. (1991). The relationship of social phobia subtypes and avoidant personality disorder. Comprehensize Psychiatry, 32, 496-502.

Shapiro, A. K. \& Morris, L. A. (1978). The placebo effect in medical and psychological therapies. In S. L. Garfield and A. E. Bergin (Eds.). Handboak of Psychotherapy and Behavior Change: An Empirical Analysis (pp. 369-410). New York: Wiley.

Shea, M. T. (1992). Some characteristics of the Axis If criteria sets and their implications for assessment of personality disorders. Joumal of Personalify Disorders, $6,377-381$.

Shea, M. T. (1993). Psychosocial treatment of personality disorders. Fournal of Personality Disorders, Stupplement, $167-180$.

Shea, M. T., Pikonis, P. A., Beckham, E., Collins, I. F, Elkin, I., Sotsky, S. M, \& Dacherty, J. P. (1990). Personality disorders and treatment of outcome in the NMH treatment of depression: Collaborative Research Program. American fournal of Psychiatry, 147, 711-718.

Shorkey, C. T. \& Whiteman, V. L. (1977). Development of the Rational Behavior Inventory: Initial validity and reliability. Educational and Psychological Measurement, 37, 527-534.

Skodol, A. E, Oldham, J. M., Rosnick, L.., Kellman, H. D. \& Hyler, S. E. (1991). Diagnoses of DSM-IIIR personality disorders: A comparison of two structured interviews. International Journal of Methods in Psychiaric Resemich, 1, 13-26.

Slavin, R. E. (1995). Bestevidence synthesis: an intelligent alternative to meta-analysis. Joumal of Chical Epidemiology, 48, 9 -18.

Spielberger, C. D., Gorsuch, R. L., \& Lushene, R. E. (1970). STAI monual for the State-Trait Anxiety Intentory. Palo Alto, Californa: Consulting Psychologists Press.

Spilzer, R. L. (1983). Psychiatric Diagnosis: Are dinicians still necessary? Comprehensive Psychiatry, 24 . $399-411$.

Spitzer, R. Lu, Forman, J. B. W., \& Nee, J. (1979). DSM-III field trials, 1 : initial interrater diagnostic reliability. American lournal of Psychiatry, $136,815-817$.

Spitzer, R. L., Williams, J. B. W., \& Gibbon, M. (1987). Structured Clinical Hntroiew for DSM-IIT-R (SCID). New York: New York State Psychiatric Institute, Biometrics Research.

Spitzer, R. L., Williams, J. B. W., Gibbon, M., \& First, M. B. (1990). Structured Clinical hatervieno for DSMHI-R Personality Disorders (SCDD-ID), Version 1.0. Washington, DC: American Psychiatric Press.

Stangl, D. Prohl, B., Zimmerman, M., Bowers, W., \& Corenthal, C. (1985). A structured interview for the DSM-III personality disorders: a preliminary report. Archioes of General Psychiatry, 42, 591-596.

Stanley, M. A. Turner, S. A. \& Borden, J. W. (1990). Schizotypal features in obsessive-compulsive disorder. Conprenersine Psychiary, 31, 511-518.

Steir, D. J.. Hollander, E., Skodol, A. E. (1993). Anxiety disorders and personality disorders: a review. Jounnal of Personality Disorders, 7, 87-104.

Steketee, G. (1990). Personality traits and disorders in obsessive-compulsives. Joumal of Anxiety Disorders, $4,351-364$ 
Sterk, W. A. \& Rijkeboer, M. M. (1997). Schenu-Vragenlijst ISchema Questionnairel Utrech University: Author:

Trull, T. J., Goodwin, A. H., Schopp, L. H., Hillenbrand, T. L. schuster, T. (1993). Psychometric properties of a cognitive measure of personality disorders. Joumal of Personality Assessment, 61 , $536-546$.

Turkat, L. D. (1985). Behazional Case Formulation. New York: Plenum.

Turner, R. M. (1987). The effects of personality disorder diagnoses on the ontrome of social anxiety symptom reduction. Journal of Personality Disorders. 1, 136-143.

Turner, S. M., Biedel, D. C., Dancu, C. V., \& Stanley, M. A. (1989). An empirically derived inventory to measure social fears and anxiety: The Social Phobia and Anxiety lnventory. Psychological Assessment: A foumal of Consulting and Clinical Psychology, 1,3540 .

Tyrer, P., Alexander, M. S. Cicchetti, D., Cohen, M. S. \& Remington, M. (1979), Reliability of a schedule for rating personality disorders. British Jounal of Psychiatry, 135, 168-174.

Tyrer, P. Casey, P. \& Gall, J. (1983). Relationship between neurosis and personality disorder. Britsh journal of Pychiatry, 142, 404-408.

Van den Hout, M. A.s Van der Molen, G. M., Griez, E., Lousberg, H. (1987). Specificily of interoceptive fear to panic disorders. Jourval of Psychopathology Behwiorat Assessment, 9, 99-106.

Van der Ploeg, H. M., Defares, P. B., \& Spielberger, C. D. (1980). Handleiding bij de Zetf-Boortulings Vragenligst [Manual of the Dutch version of the STAI]. Ansterdam/Lisse: Swets \& Zeitlinger.

Van Velzen, C. J. M. Emmelkamp, P. M. G., \& Scholing. A. (1997). The impact of personality disorders on behavioral treatment outcome for social phobia. Behnoiour Researd and Therapy, 35, 889-900.

Van Velzen, C. J. M. \& Emmelkamp, P. M. G. (1996). The assessment of personality disorders: implications for cognitive and behavior therapy. Behawiour Research and Therapy, 34, 655-668.

Van Velzen, C. J. M., Emmelkamp, P. M. G. Scholing, A., Luteijn, F. (1995). The influence of personaliny disorders on treatment outcome of social phobia: a preliminary report. Paper presented at the World Congress of Behavioural and Cognitive Therapies, Copenhagen, 1995.

Vandiver, T. \& Sher, K. J. (1991). Temporal stability of the Diagnostic Interview Schedule Psychological Assessment: Joumal of Consuling and Clinical Psychology, 3, $277-281$.

Vaughan, M. \& Beech, H.. R. (1985). Which obsessionals tail to change. In D. T. Mays \& C. M. Franks (eds.): Negatioe Outcome in Psychotherapy and What To Do About It (pp. 195-198). New York: Springer.

Watson, J. P. \& Marks, I. M. (1971). Relevant and irrelevant fear and flooding. Behazior Theropy, 2, 275293.

Weaver, T. L. \& Clum, G. A. (1993). Early family enwironments and traumatic experiences associated with borderline personality disonder. Joumal of Consulting and Clinical Psychology, 61, 1068-1075.

Weertman, A. Arntz, A., Kerkhofs, M. L. M. (1997). Nederlandse vertaing wan het Gestructureerd Klim wisch Interview voor DSM-IV persconlijkheidsstoornissem (SCID-II)(Dutch translation of the Structured Clinical Interview for DSM-IV personality disorders (SCID-II)]. Maastricht Universily: Author.

Weertman, A., Dreessen, L., \& Arntz. A. (1998). Personality disorders and information processing: assessing the interpretation of ambiguous information using the Thematic Apperception Test. Manuscrips in preparation.

Widiger, T. A. \& Frances, A. (1987). Interviews and inventories for the measurement of personality disorders. Clinical Psychology Review, 7, 49-75.

Widiger, T. A. (1992). Categorical versus dimensional classification: implications from and for research. joumal of Personality Disorders, $6,287-300$.

Widiger, T. A. (1993). Vallidation strategies for the personality disorders. Joumal of Personaliny Disorders, Supplement, Spring, 34-43. 
Williams, I. M. G. Watts, F. N. Macleod, C., \& Mathews, A. (1988). Cogritive Psychology and Emotional Disorders. Wiley Series in Clinical Psychology. New York: Wiley.

Winters, K C. \& Neale, J. M. (1985). Mania and low self-esteem. Journal of Abromal Psychology, 94,282 290.

Wonderlich, S. A, Swift, W. Y., Slotnick, H. B., \& Goodman, S. (1990). DSM-IIL-R personaliiy disordiers in eating-disorder subtypes. International Joumal of Eating Disorders, 9, 607-616.

Woody, G. E. Mclellan, A. T, Luborsky, L., \& O'Brien, C. P. (1985). Sociopathy and psychotherapy outcome. Archroes of Gereral Psychiatry, 42, $1081-1086$.

Young, J. E. (1990). Cognitive Therapy for Personality Disorders: A Schema-focused Approach. Sarasota: Professional Resource Press.

Zanarini, M. C. Frankenburg. F. R., Chauncey, D. L., \& Gunderson, J. G. (1987). The Diagnostic Interview for Personality Disorders: interrater and test-retest reliability. Compreherisive Psychiatry, 28, $467-480$.

Zimmerman, M. (1994). Diagnosing personality disorders. A review of issues and research rnethods. Archires of General Psychialry, 51, 225-245.

Zimmerman, M. \& Coryell, W. (1989). DSM-III personality disorder diagnoses in a nonpatient sample. Archines of General Psychiatry, 46, 682-689.

Zimmerman, M., Pfohl ${ }_{*}$ B., Coryell, W., Stangl, D., \& Corenthal, C. (1988). Diagnosing personality disorder in depressed patients. Archives of General Psychiatry, 45, 733-738. 


\section{Summary}

This thesis comprises research into (a) the assessment of personality disorders, (b) the impact of personality disorders on treatment of anxiety disorders, and (c) the cognitive profiles of personality disorders. Throughout the thesis, personality disorders are defined according to the Diagnostic and Statistical Manual of Mental Disorders (DSM) published by the American Psychiatric Association.

Chapter 1 offers an introduction to the following chapters. First, various difficulties in the assessment of personality disorders are discussed. It is argued that further tests for reliability and validity are needed. Next, the possible impact of personality disorders on therapy-process and therapy-outcome of anxiety disorders is addressed. It is concluded that therapy-process studies are lacking and that the many therapy-outcome studies suffer from methodological limitations. Finally, the cognitive theory of personality disorders is detailed. This promising theory needs to be validated by studying the hypothesized information processing biases and by developing theory based assessment instruments.

Chapters 2, 3 and 5 report on several empirical studies. Chapter 4 contains a review article and Chapter 5 reports on a new belief questionnaire for personality disorders. The major findings and conclusions can be summarized as follows:

1. In a group of outpatients, the test-retest interrater reliability of the Structured Clinical Interview for DSM-III-R Personality Disorders (SCID-II) was found to be satisfactory, except for the histrionic personality traits (section 2.1).

2. The validity of patient reports on personality pathology, which can be questioned for several reasons, was studied by comparing patient based and informant based SCID-II diagnoses (convergent validity). Similar to prior studies, which used other assessment instruments for personality disorders, low or only modest levels of agreement were found. In this study, it was attempted to determine which of the two sources of information was more valid. Comparisons with therapist based diagnoses suggested that patient reports were more valid than informant reports, but no final conclusions can be drawn yet (section 2.2).

3. The impact of SCID-II personality disorder variables (based on patient interviews) on outcome of cognitive and behavior therapy (CBT) for anxiety disorders was studied in three groups of patients: (a) patients with an anxiety disorder, irrespective of kind, receiving individually tailored CBT; (b) patients with panic disorder, receiving standardized $C B T$; and (c) patients with obsessive compulsive disorder, receiving standardized CBT. Categorical personality disorder variables did not affect the amount of improvement in the short term (general as well as several specific personality disorder variables were studied). The evaluation of dimensional personality disorder variables showed similar results, except for obsessive compulsive traits. These traits were related to less Axis I improvement in a group of patients that was treated for panic disorder and in a group of patients that was treated for different anxiety disorders. None of the three studies showed evidence for patients with personality pathology to 
select themselves out of therapies for anxiety disorders, once they had started. In the obsessive compulsive disorder study, data from the drop-outs were included in the analyses but still personality disorder variables did not affect CBT outcome negatively. Patients with panic disorder and obsessive compulsive disorder were followed up until 6 months after the standardized therapy had finished, and the promising short-term results were maintained. The strengths of the three studies are the prospective design, the use of a semistructured interview for personality disorders, and the quantification of improvement as change with respect to pretest scores (sections 3.1 and 3.2).

4. The research literature on the impact of personality disorder variables on outcome of therapies for anxiety disorders was reviewed. 15 studies out of 35 were selected, because they met the two a priori selection criteria (prospective design and use of a semistructured interview for personality disorders). From the selected studies it was concluded that, in general, personality disorder variables had no robust negative impact on treatment outcome of anxiety disorders. However, some specific personality disorder variables, such as avoidant, schizotypal, and obsessive compulsive personality traits, are suspect and should be studied further (Chapter 4 ).

5. SCID-II personality pathology, based on patient interviews by independent raters, was not related to an excessively and insurmountably negative impact on the therapy-process of $\mathrm{CBT}$ for anxiety disorders (as reported by the therapist). This finding is consistent with the findings that personality disorders do not affect outcone in CBT for anxiety disorders. On the contrary, personality pathology as evaluated by the therapist was strongly related to a dysfunctional therapy process. The question presents itself whether therapists did or did not rush to premature conclusions in attributing failure in the therapy process to assumed concomitant personality pathology (section 3.3).

6. The Personality Disorder Belief Questionnaire ( $\mathrm{PDBQ}$ ) was introduced as a new cognitive measure for personallity disorders, to be used in empirical studies and clinical practice. Preliminary data on psychometric properties were found to be encouraging enough to continue further research on reliability and validity (section 5.1).

7. As predicted from cognitive theory, DSM-HI-R avoidant personality pathology was associated with avoidant beliefs (measured by the PDBQ), and, using a pragmatic inference task, avoidant beliefs were associated with a schemacongruent information processing bias. However, DSM-III-R avoidant personality pathology was not associated with such a bias, which might be explained by methodological weaknesses of the study and/or by theoretical issues. In addition to avoidant beliefs, self-esteem was also associated with this bias. Both effects remained present after controlling for several relevant variables, and this was considered an indication of the specificity of the effects found (section 5.2).

Finally, Chapter 6 offers a synthesis of the previous chapters. The major findings are discussed and suggestions for further research on personality disorders are given. The chapter ends with recommendations for clinical practice. Suggestions for further research are, for instance: the use of a short-interval test-retest design 
in studying interrater reliability of (semi)structured interviews for personality disorders; the use of external criteria against which to compare patient and informant based personality disorder diagnoses; the use of clear hypotheses to select specific personality disorder variables when studying their impact on Axis I treatment outcome and process; and the validation of the cognitive theory and therapy of personality disorders. The most important dinical recommendations are that patients with personality disorders should not be excluded in advance from CBT for their Axis I anxiety disorder, and that therapists should be careful in attributing Axis $r$ therapy failure to DSM-MII-R personality disorders. 



\section{Samenvatting}

Dit proefschrift bevat onderzoek naar (a) de diagnostiek van persoonlijkheidsstoornissen, (b) de invloed van persoonlijkheidsstoomissen op de behandeling van angststoornissen, en (c) de cognitieve profielen van persoonlijkheidsstoornissen. In het gehele proefschrift worden persoonlijkheidsstoornissen gedefiniëerd volgens de Diagnostic and Statistical Manual of Mental Disorders (DSM) uitgegeven door de American Psychiatric Association.

Hoofdstuk 1 is een inleiding op de daarop volgende hoofdstukken. Eerst worden de verschillende moeilijkheden die zich voordoen in de diagnostiek van persoonlijkheidsstoornissen besproken. Daarbij wordt de noodzaak van verder onderzoek naar de betrouwbaarheid en validiteit bepleit. Vervolgens wordt de mogelijke invloed van persoonlijkheidsstoornissen op het therapie-proces en het therapie-effect bij angststoornissen besproken. Er wordt vastgesteld dat studies over therapieproces ontbreken en dat de talrijke studies over therapie-effect methodologische tekortkomingen vertonen. Tenslotte wordt de coginitieve theorie over persoonlijkheidsstoornissen nauwkeurig beschreven. Deze veelbelovende theorie moet worden getoetst door de veronderstelde vertekeningen in de informatieverwerking te onderzoeken en door op de theorie gebaseerde meetinstrumenten te ontwikkelen.

In de hoofdstukken 2,3 , en 5 worden diverse empirische studies beschreven. Hoofdstuk 4 bevat een overzichtsartikel en in hoofdstuk 5 wordt een nieuwe assumptievragenlijst voor persoonlijkheidsstoornissen gepresenteerd. De belangrijkste bevindingen en conclusies kunnen als volgt worden samengevat:

1. In een groep poliklinische patiënten bleek de test-hertest interbeoordelaarsbetrouwbaarheid van het Structured Clinical Interview for DSM-IM-R Personality Disorders (SCID-II) toereikend te zijn, met uitzondering van de theatrale persoonlijkheidstrekken (sectie 2.1).

2. De validiteit van patiënt-rapportages over persoonlijkheidspathologie, die om verschillende redenen in twijfel kan worden getrokken, werd onderzocht door SCID-II diagnoses op basis van patiënt-interviews te vergelijken met SCID-II diagnoses op basis van informant-interviews (convergente validiteit). In overeenstemming met eerdere studies, die andere meetinstrumenten voor persoonlijkheidsstoornissen gebruikten, werd lage of slechts matige overeenstemming gevonden. In deze studie werd geprobeerd om vast te stellen welke informatiebron meer valide is. De vergelijkingen met de therapeutoordelen over persoonlijkheidspathologie suggereerden dat patiënt-rapportages meer valide zijn dan informant-rapportages, maar er kunnen nog geen definitieve conclusies worden getrokken (sectie 2.2).

3. De invloed van SCID-II persoonlijkheidsstoornis-variabelen (gebaseerd op patiënt-interviews) op het effect van cognitieve therapie en ged ragstherapie (CBT) bij angststoornissen werd onderzocht in drie groepen patienten: (a) patienten met een angststoornis, ongeacht het type, die een op het individu afgestemde, ongestandaardiseerde CBT kregen; (b) patiënten met een paniekstoornis, die 
een gestandaardiseerde CBT kregen; en (c) patiënten met een obsessievecompulsieve stoornis, die een gestandaardiseerde CBT kregen. Categoriale persoonlijkheidsstoornis-variabelen hadden geen invloed op de mate van vooruitgang op korte termin (daarbij werden zowel algemene als specifieke persoonlijkheidsstoornis-variabelen bekeken). De analyses van dimensionele persoonlijkheidsstoornis-variabelen lieten gelikaardige resultaten zien, met uitzondering van de obsessieve-compulsieve trekken. Deze trekken hingen samen met minder vooruitgang bij de groep patiènten die werd behandeld voor een paniekstoornis en bij de groep patiënten die werd behandeld woor verschillende angststoornissen. Uit geen van de drie studies bleek dat patiënten met persoonlijkheidspathologie, als ze eenmal zijn begonnen, voortijdig stoppen met een behandeling voor de angststoornis. In de studie naar de obsessieve-compulsieve stoornis werden de gegevens van de uitvallers geschat en betrokken in de analyses en nog steeds hadden persoonlijkheidsstoornisvariabelen geen negatieve invloed op het effect van CBT. De groep patiënten met een paniekstoornis en de groep met een obsessieve-compulsieve stoomis werden na afloop van de gestandaardiseerde behandeling 6 maanden gevolgd, en de veelbelovende korte-termijn effecten bleven gehandhaafd. De sterke punten van de bovengenoemde drie studies zijn het prospectieve design, het gebruik wan een semigestructureerd interview voor persoonlijkheidsstoornissen, en het operationaliseren van vooruitgang als verandering ten opzichte van de voormeting (secties 3.1 en 3.2 ).

4. Er werd een overzicht gegeven van de onderzoeksliteratuur naar de invloed van persoonlijkheidsstoornis-variabelen op het effect van behandelingen voor angststoornissen. 15 van de 35 onderzoeken werden geselecteerd, omdat ze voldeden aan twee vooraf bepaalde selectiecriteria (prospectief design en gebruik van een semigestructureerd interview voor persoonlijkheidsstoomissen). Op basis van de geselecteerde studies kon worden geconcludeerd dat, over het algemeen, persoonlijkheidsstoornis-variabelen geen sterk negatieve invloed hadden op het therapie-effect bij angststoomissen. Echter, de invloed van sommige specifieke persoonlijkheidsstoomis-variabelen, zoals de ontwijkende, schizotypische and obsessieve-compulsieve persoonlijkheidstrekken, is twij" felachtig en dient verder onderzocht te worden (Hoofdstuk 4).

5. SCID-I persoonlijkheidspathologie (gebaseend op patient-interviews door onahankelijke beoordelaars) had geen sterk en onoverkomelijk negatieve inwloed op het therapie-proces van CBT bij angststoornissen (zoals geëvalueerd door de therapeut). Deze bevinding is in overeenstemming met de bevindingen dat persoonlijkheidsstoornissen geen invloed hebben op het effect van CBT bij angst stoornissen. De oordelen wan de therapeut over de persoonlijkheidspathologie, daarentegen, correleerden sterk met een dysfunctioneel therapieproces. De vraag doet zich voor of therapeuten al dan niet overhaste conclusies hebben getrokken door een dysfunctioneel therapieproces toe te schrijven aan vermeende persoonlijkheidspathologie (sectie 3.3).

6. De Personality Disorder Belief Questionnaire (PDBQ) werd geïtroduceerd als een nieuw cognitief meetinstrument voor persoonlijkheidsstoomissen, dat gebruikt kan worden in empirische studies en in de klinische praktijk. Voorlopige data over psychometrische eigenschappen bleken bemoedigend genoeg te zijn om 
verder onderzoek naar de betrouwbaarheid en validiteit voort te zetten.

7. Zoals voorspeld door de cognitieve theorie, hing DSM-III-R ontwijkende persoonlijkheidspathologie samen met ontwijkende assumpties (gemeten met de $P D B Q$ ), en, gebruikmakend van een pragmatische inferentie-taak, bleek dat ontwijkende assumpties samenhingen met een schema-congruente vertekening in de informatieverwerking. Echter, DSM-III-R ontwijkende persoonlijkheidspathologie hing niet samen met een dergelijke vertekening, wat mogelijk kan worden verklaard door zwakke punten in de methodologie van de studie en/ of door theoretische kwesties. Behalve ontwijkende assumpties, hing ook lage zelfwaardering samen met de vertekening. Beide verbanden bleven aanwezig na controle voor verschillende relevante variabelen, en dit werd gezien als een aanwijzing voor de specificiteit van de gevonden verbanden (sectie 5.1).

Tenslotte worden de voorafgaande hoofdstukken in hoofdstuk 6 geïtegreerd. De belangrijkste bevindingen worden bediscussiëerd en er worden suggesties gedaan voor verder onderzoek naar persoonlijkheidsstoornissen. Het hoofdstuk eindigt met aanbevelingen voor de klinische praktijk. Suggesties voor verder onderzoek zijn bijvoorbeeld: het gebruik van een test-hertest design in het onderzoek naar de interbeoordelaarsbetrouwbaarheid van (semi)gestructureerde interviews voor persoonlijkheidsstoornissen; het gebruik van externe criteria in de vergelijking tussen patiënt- en informant rapportages over persoonlijkheidspathologie; het hypothese-gestuurd selecteren van specifieke persooniijkheidsstoomis-variabelen in het onderzoek naar de invloed op het proces en het effect van As I behandelingen; en de validering van de cognitieve theorie en therapie van persoonlijkheidsstoornissen. De belangrijkste klinische aanbevelingen zijn dat patiënten met persoonlijkheidsstoornissen niet bij voorbaat uitgesloten moeten worden van $C B T$ voor hun As I angststoornis, en dat therapeuten terughoudend moeten zijn om falen in de As I therapie toe te schrijven aan DSM-III-R persoonlijkheidsstoornissen. 



\section{Dankwoord}

Dit proefschrift is het resultaat van de inspanningen van vele mensen. Allereerst wil ik alle mensen bedanken die deelgenomen hebben aan de onderzoeken. Het grootste deel van de gegevens is afkomstig van mensen die in een moeilijke periode van hun leven toch bereid waren om te investeren in wetenschappelijk onderzoek. Mijn speciale dank hiervoor.

Arnoud Antz, co-pronotor. Jouw onmisbare bijdrage aan dit proefschrift waardeer ik enorm. Het begon allemaal toen ik in mijn studententijd samen met jou onderzoek kon doen. Die samenwerking heeft er zeker toe bijgedragen dat ik daarna koos voor promotie-onderzoek. Ik vind dat ik geboft heb met een begeleider zoals jij: je weet veel en je bent snel, kritisch, enthousiast en steunend in je commentaar.

Marcel van den Hout, promotor. Het was goed om te weten dat jij altijod bereikbaar was. Het was niet nodig om daar heel intensief gebruik van te maken. Jij was voor mij vooral een inspiratiebron op afstand en een baas met een hart voor zijn mensen.

Alle leden van de bevordelingscommissie. Ik bedank Harald Merckelbach, Wim van den Brink, Herman van Praag, Corine de Ruiter en Henk Schmidt voor hun bereidheid om zitting te nemen in de beoordelingscommissie.

Alle collega's van het Departement Medische, Klinische en Experinentele Psychologie en het instituut Experimentele Psychopathologie. Ik dank jullie voor het bieden van een inspirerende werkkring. Goede voorbeelden doen goed volgen. Het was bijzonder om te ervaren dat ik deel uitmaakte van een hechte groep enthousiaste werkers. Voor hun directe bijdrage aan dit proefschrift wil ik bedanken: Erik Schouten, voor zijn engelengeduld als ik weer eens binnenliep met een beklemmende statistische vraag; Ger van. Wunnik voor de audio-opnames; de secretariaatmedewerksters voor hun ondersteuning bij de administratie rondom het proefschrift; en tenslotte de dames en heren promovendi: het is veel leuker om samen in een wak te zitten dan alleen, en het is heerlijk om collectief de lasten te externaliseren en de lusten intern te attribueren.

Alle collega's wan de RIAGG Marastricht. Ik dank jullie allen voor het bieden van een tweede, wederom fantastische werkplek. Allereerst wil ik alle collega's van het Dwarsverband Academisering (DAC) bedanken voor hun medewerking aan de uitvoering van de meeste onderzoeken in dit proefschrift. De onderzoeksassistenten hebben veel onderzoeksgegevens verzameld en ingevoerd. Hanny Keijsers wil ik in het bijzonder bedanken. Dankzij haar bezielde leiding verliep de dataverzameling gesmeerd. De therapeuten hebben interviews afgenomen, vragenlijsten ingevuld, protocollaire behandelingen uitgevoerd en cliënten gemotiveerd om mee te doen aan mijn onderzoek. Ik heb veel respect voor jullie inzet ten behoeve van de academisering. Brigit Lok wil ik bedanken voor alle secretariële ondersteuning en Rense Hoekstra voor zijn bijdrage als coördinator-onderzoek van het DAC ten tijde van dit promotie-onderzoek. Alle collega's van het DAC hebben ook gezorgd voor een optimaal groeiklimaat voor een jonge therapeute. Dit 
lijkt geen werband te hebben met dit proefschrift, maar voor mij is therapie geven en onderzoek doen sterk met elkaar verbonden. Alle collega's van de Afdeling Psychotherapie wil ik bedanken voor het feit dat ik me op deze afdeling welkom voelde als ik in mijn rol als therapeut kroop en mijn rol als onderzoeker achter me liet. Alle collega's van de afdeling Kinder-en Jeugdzorg wil ik bedanken voor de oprechte belangstelling voor de perikelen rond de afronding van mijn proefschrift. De directie wil ik bedanken voor het mogelijk maken van onderzoek binnen het DAC.

Studenten GGK, scriptieschrijuers. Martin Hildebrand, Nancy Keune, Tatja Hendriks en Anoek Weertman, bedankt voor de vele uren denkend en uitvoerend werk bij de onderzoeken van dit proefschrift.

Lisbeth Hall. Bedankt voor je correcties in mijn Engels.

Saydra Daenns. Je nam een last van mijn schouders door het proefschrift te layouten.

Tenslotte, mijn familieleden, wrienden en geliefde. Jullie belangstelling, steun, begrip en waardering deden mij goed. Een aantal van jullie wil ik bij naam noemen. Anja, kamergenote, vriendin en paranimf, ik heb zoveel aan je gehad. We zaten op dezelfde golflengte en achter de gesloten deur van onze werkkamer werden bellangrijke levenskwesties besproken. Erik, vriend en paranimf, fijn dat je, net als Anja, mee wilde helpen bij de organisatie van de plechtigheid en het feest, en achter mij op het podium wil staan. Pap en mam, jullie hebben me de vrijheid gegeven om te studeren en altijd vertrouwen gehad in mijn keuzes. Jullie steun betekende veel voor mij. Rob, jij stond al die jaren het dichtste bij me. Ik maakte je deelgenoot van mijn ongecensureerde vreugde en verdriet in dit spel. Tijdens de zware momenten hield jij het hoofd koel en bleef je aan mijn zijde. Daar heb ik veel aan gehad. Bedankt! 


\section{Curriculum vitae}

De schrijfster van dit proefschrift werd in 1968 in Bemelen geboren. In 1986 behaalde zij het vwo-diploma aan het Jeanne d'Arc College te Maastricht en begon zij met de studie Gezondheidswetenschappen aan de Universiteit Maastricht. Tijdens deze studie was ze student-assistent bij de Universiteit Maastricht en onderzoeks-assistent bij de RIAGG Maastricht. In 1991 behaalde zij het doctoraal diploma met als afstudeerrichting Geestelijke Gezondheidkunde. Aansluitend ging zij werken als Junior-Gedragstherapeut bij het Dwarsverband Academisering bij de RIAGG Maastricht en startte zij met de RINO-opleiding tot psychotherapeut en met de vGT-opleiding tot gedragstherapeut. Vanaf december 1991 tot december 1996 was zij werkzaam als Assistent-In-Opleiding bij de vakgroep Differentiële en Experimentele Psychologie, Universiteit Maastricht, met een detachering als therapeut-in-opleiding naar het Dwarsverband Academisering, RLAGG Maastricht. Dit proefschrift volgt uit die periode. Vanaf december 1996 is zij als universitair docent verbonden aan het Departement Medische, Klinische en Experimentele Psychologie, Universiteit Maastricht, alwaar zij naast het geven van onderwijs binnen de faculteit Geneeskunde, onderzoek verricht naar psychopathologie bij kinderen en jeugdigen. Daarnaast heeft $\mathrm{zij}$, in het kader van academisering, organiserende en psychotherapeutische taken op de afdeling Kinder-en Jeugdzorg, RIAGG Maastricht. Zij is inmiddels registreerd psychotherapeut en gedragstherapeut. 